\title{
Rhodium-Catalyzed C=N Bond Formation through a Rebound Hydrolysis Mechanism and Application in $\beta$-lactam Synthesis
}

\author{
Long Chen, ${ }^{+, \S}$ Linxing Zhang,,$\$$ Ying Shao, ${ }^{\dagger}$ Guangyang Xu, ${ }^{\dagger}$ Xinhao Zhang, ${ }^{,+,, \pi}$ \\ Shengbiao Tang ${ }^{\dagger}$ and Jiangtao Sun ${ }^{*}$,
}

†Jiangsu Key Laboratory of Advanced Catalytic Materials \& Technology, School of Petrochemical Engineering, Changzhou University, Changzhou 213164, China

${ }^{*}$ Lab of Computational Chemistry and Drug Design, State Key Laboratory of Chemical Oncogenomics, Peking University Shenzhen Graduate School, Shenzhen 518055, China

'Shenzhen Bay Laboratory, Shenzhen, 518055, China

E-mail: zhangxinhao@pku.edu.cn

jtsun@cczu.edu.cnorjtsun08@gmail.com

Table of Contents

1. General information

2. Optimization of the reaction conditions for $\mathbf{5 a}$

3. General procedure for Scheme 2

4. General procedure for Scheme 4

5. Preparation of $\mathbf{3 a}$ in $2 \mathrm{mmol}$ scale and 5a in $1 \mathrm{mmol} \mathrm{scale}$

6. X-ray structure of $\mathbf{5 q}$ and $\mathbf{5 s}$

7. References

8. ${ }^{1} \mathrm{H}$ NMR and ${ }^{13} \mathrm{C}$ NMR Spectra of compounds

9. Density functional theory (DFT) calculations 


\section{General information}

All of the reactions were carried out in flame-dried tubes under argon atmosphere. Solvents were dried prior to use. For column chromatography, 200-300 mesh silica gel was used. ${ }^{1} \mathrm{H}$ NMR were recorded on Bruker $300 \mathrm{MHz}$ or $400 \mathrm{MHz}$ spectrometer and ${ }^{13} \mathrm{C}$ NMR were recorded on Bruker $75 \mathrm{MHz}, 100 \mathrm{MHz}$ or $125 \mathrm{MHz}$ spectrometer in $\mathrm{CDCl}_{3}$ or DMSO- $\mathrm{d}_{6}$. Chemical shifts are expressed in parts per million (ppm) and reported relative to $\mathrm{CDCl}_{3}$ peaks $\left[\delta=7.26 \mathrm{ppm}\left({ }^{1} \mathrm{H} \mathrm{NMR}\right) ; \delta=77.1 \mathrm{ppm}\left({ }^{13} \mathrm{C} \mathrm{NMR}\right)\right]$ or DMSO-d 6 peaks $\left[\delta=2.50 \mathrm{ppm}\left({ }^{1} \mathrm{H}\right.\right.$ NMR $\left.) ; \delta=40.0 \mathrm{ppm}\left({ }^{13} \mathrm{C} \mathrm{NMR}\right)\right]$. Multiplicities are denoted as follows: $\mathrm{s}=$ singlet, $\mathrm{d}=$ doublet, $\mathrm{t}=$ triplet, $\mathrm{q}=$ quartet, $\mathrm{dd}=$ doublet of doublets, and $\mathrm{m}=$ multiplet. Coupling constants $(J)$ are reported in Hertz (Hz). HRMS were performed on Agilent 6540 Q-TOF mass spectrometer (ESI). Melting points were determined on a SGW X-4B melting point apparatus.

The N-hydroxylanilines $1,{ }^{1}$ diazo compounds $2^{2}$ and diazocarbonyl compounds $4^{3}$ were prepared according to the literature procedures.

\section{Optimization of the reaction conditions for $5 a^{[a]}$}

\begin{tabular}{lllll} 
Entry & Catalysts & Solvent & Time (h) & Yield(\%) \\
\hline 1 & {$\left[\mathrm{CP}^{\mathbf{b}} \mathrm{RhCl}_{2}\right]_{2}$} & $\mathrm{DCM}$ & 3 & 47 \\
2 & {$\left[\mathrm{Rh}_{2}(\mathrm{COD}) \mathrm{Cl}\right]$} & $\mathrm{DCM}$ & 3 & trace \\
3 & $\mathrm{Rh}_{2}(\mathrm{esp})_{2}$ & $\mathrm{DCM}$ & 3 & 51 \\
4 & $\mathrm{Rh}_{2}(\mathrm{oct})_{4}$ & $\mathrm{DCM}$ & 3 & 9 \\
5 & $\mathrm{Rh}_{2}(\mathrm{OPiv})_{4}$ & $\mathrm{DCM}$ & 3 & 44 \\
6 & $\mathrm{Rh}_{2}(\mathrm{OAc})_{4}$ & $\mathrm{DCM}$ & 3 & 83 \\
7 & $\mathrm{Cu}_{(\mathrm{OAc})_{2}}$ & $\mathrm{DCM}$ & 3 & $\mathrm{ND}$ \\
8 & $\mathrm{Cu}_{(\mathrm{OTf})_{2}}$ & $\mathrm{DCM}$ & 3 & $\mathrm{ND}$ \\
9 & $\mathrm{Rh}_{2}(\mathrm{OAc})_{4}$ & $\mathrm{DCE}$ & 3 & 53 \\
10 & $\mathrm{Rh}_{2}(\mathrm{OAc})_{4}$ & $\mathrm{CHCl}$ & 3 & 46 \\
11 & $\mathrm{Rh}_{2}(\mathrm{OAc})_{4}$ & $\mathrm{THF}$ & 3 & 42 \\
12 & $\mathrm{Rh}_{2}(\mathrm{OAc})_{4}$ & $\mathrm{MeCN}$ & 3 & 32 \\
\hline
\end{tabular}




\begin{tabular}{lllll}
\hline 13 & $\mathrm{Rh}_{2}(\mathrm{OAc})_{4}$ & Toluene & 3 & 54 \\
14 & $\mathrm{Rh}_{2}(\mathrm{OAc})_{4}$ & $\mathrm{DMF}$ & 3 & 38 \\
15 & & $\mathrm{DCM}$ & 3 & trace \\
\hline
\end{tabular}

[a] Reaction conditions: 1a (0.2 mmol, 1 equiv), 2a (0.24 mmol,1.2 equiv), 4a (0.36 mmol, 1.8 equiv) and catalyst $(1 \mathrm{~mol} \%)$ in solvent $(4 \mathrm{~mL})$ at $\mathrm{rt}$ for $3 \mathrm{~h}$. [b] Isolated yield.

\section{General procedure for Scheme 2}

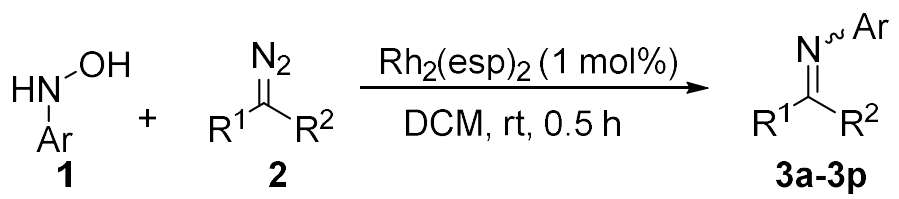

To a tube was added $\mathrm{Rh}_{2}(\mathrm{esp})_{2}(0.002 \mathrm{mmol}, 0.01 \mathrm{eq}), 1$ (0.2 mmol, $\left.1 \mathrm{eq}\right), 2$ (0.24 mmol, $1.2 \mathrm{eq})$ and DCM (4 mL) under argon atmosphere, the resulting solution was stirred at $\mathrm{rt}$ for $0.5 \mathrm{~h}$, [Note: $\mathbf{3 m}$ and $\mathbf{3 p}$ were performed at $80{ }^{\circ} \mathrm{C}$ ]. The reaction mixture was concentrated under vacuum, the residue was purified by column chromatography (silica gel, eluted with EtOAc: Petroleum ether $=1: 20$ ) to give product 3a-3p.

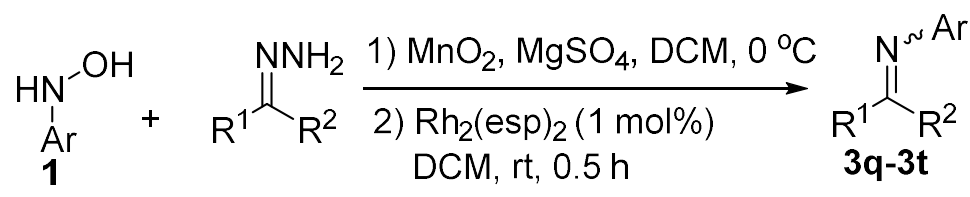

To a mixture of hydrazone $(0.24 \mathrm{mmol}, 1.2 \mathrm{eq})$ and $\mathrm{MgSO}_{4}(0.3 \mathrm{mmol}, 1.5 \mathrm{eq})$ in $\operatorname{DCM}(3 \mathrm{~mL})$ was added $\mathrm{MnO}_{2}(1.44 \mathrm{mmol}, 7.2 \mathrm{eq})$ at $0{ }^{\circ} \mathrm{C}$ under argon atmosphere, the mixture was stirred for $1 \mathrm{~h}$. The mixture was filtered, the purple filtrate was added into a solution of $\left(\mathrm{Rh}_{2}(\mathrm{esp})_{2}(0.002 \mathrm{mmol}, 0.01 \mathrm{eq})\right.$ and $1(0.2 \mathrm{mmol}, 1 \mathrm{eq})$ in DCM (1 $\mathrm{mL}$ ) under argon atmosphere, then the resulting solution was stirred at $\mathrm{rt}$ for $0.5 \mathrm{~h}$. The reaction solution was concentrated under vacuum, the residue was purified by column chromatography (silica gel [Note: the silica gel was pretreated with TEA], eluted with EtOAc: Petroleum ether $=1: 100)$ to give product $\mathbf{3 q - 3 t}$.<smiles>CC(=O)/C(=N\c1ccccc1)c1ccccc1</smiles> 


\section{Methyl 2-phenyl-2-(phenylimino)acetate (3a)}

The title compound was prepared via general procedure using $21.8 \mathrm{mg}$ of N-phenylhydroxylamine (1a), 3a was isolated as yellow oil $(45.0 \mathrm{mg}$, a mixture of geometric isomers (94:6), 94\%). ${ }^{1} \mathrm{H}$ NMR (400 $\left.\mathrm{MHz}, \mathrm{CDCl}_{3}\right)$ major isomer $\delta$ 7.91-7.83 (m, 2H), 7.54-7.44 (m, 3H), 7.34 (t, $J=7.7 \mathrm{~Hz}, 2 \mathrm{H}), 7.14$ (t, $J=7.4 \mathrm{~Hz}$, $1 \mathrm{H}), 6.96(\mathrm{~d}, J=7.4 \mathrm{~Hz}, 2 \mathrm{H}), 3.63(\mathrm{~s}, 3 \mathrm{H}) .{ }^{13} \mathrm{C} \mathrm{NMR}\left(100 \mathrm{MHz}, \mathrm{CDCl}_{3}\right) \delta 165.5$, $160.0,150.1,133.8,131.9,128.9,128.8,128.0,125.0,119.5,51.9$.<smiles>COC(=O)/C(=N/c1ccccc1C)c1ccccc1</smiles>

\section{Methyl 2-phenyl-2-(o-tolylimino)acetate $(3 b)^{5}$}

The title compound was prepared via general procedure using $24.6 \mathrm{mg}$ of $\mathrm{N}-(o$-tolyl)hydroxylamine (1) $), \mathbf{3 b}$ was isolated as yellow oil $(47.1 \mathrm{mg}$, a mixture of geometric isomers (95:5), 93\%). ${ }^{1} \mathrm{H}$ NMR (400 $\left.\mathrm{MHz}, \mathrm{CDCl}_{3}\right)$ major isomer $\delta$ 7.94-7.85 (m, 2H), 7.55-7.44 (m, 3H), 7.19 (d, $J=7.4 \mathrm{~Hz}, 1 \mathrm{H}), 7.15-7.08(\mathrm{~m}, 1 \mathrm{H})$, 7.07-7.01 (m, 1H), $6.71(\mathrm{~d}, J=7.4 \mathrm{~Hz}, 1 \mathrm{H}), 3.60(\mathrm{~s}, 3 \mathrm{H}), 2.22(\mathrm{~s}, 3 \mathrm{H}) .{ }^{13} \mathrm{C}$ NMR $(100$ $\left.\mathrm{MHz}, \mathrm{CDCl}_{3}\right) \delta 165.4,159.4,149.0,133.7,131.8,130.2,129.2,128.8,128.0,126.2$, $124.9,117.4,51.9,18.0$.<smiles>COC(=O)/C(=N/c1cccc(C)c1)c1ccccc1</smiles>

\section{Methyl 2-phenyl-2-(m-tolylimino)acetate (3c)}

The title compound was prepared via general procedure using $24.6 \mathrm{mg}$ of $\mathrm{N}$-(m-tolyl)hydroxylamine (1c), 3c was isolated as yellow oil $(47.6 \mathrm{mg}$, a mixture of geometric isomers (95:5), 94\%). ${ }^{1} \mathrm{H}$ NMR (300 $\left.\mathrm{MHz}, \mathrm{CDCl}_{3}\right)$ major isomer $\delta$ 
7.91-7.79 (m, 2H), 7.53-7.42 (m, 3H), $7.21(\mathrm{t}, J=5.7 \mathrm{~Hz}, 1 \mathrm{H}), 6.95(\mathrm{~d}, J=5.7 \mathrm{~Hz}$, 1H), $6.81(\mathrm{~s}, 1 \mathrm{H}), 6.76(\mathrm{~d}, J=5.7 \mathrm{~Hz}, 1 \mathrm{H}), 3.64(\mathrm{~s}, 3 \mathrm{H}), 2.34(\mathrm{~s}, 3 \mathrm{H}) .{ }^{13} \mathrm{C}$ NMR $(75$ $\left.\mathrm{MHz}, \mathrm{CDCl}_{3}\right) \delta 165.6,159.7,150.0,138.8,133.9,131.8,128.8,128.0,125.9,120.4$, 116.4, 51.9, 21.5. HRMS (ESI) calculated for $\mathrm{C}_{16} \mathrm{H}_{16} \mathrm{NO}_{2}[\mathrm{M}+\mathrm{H}]^{+}: 254.1176$, found: 254.1171 .<smiles>CC(=O)/C(=N/c1ccc(C)cc1)c1ccccc1</smiles>

Methyl 2-phenyl-2-(p-tolylimino)acetate $(3 d)^{5}$

The title compound was prepared via general procedure using $24.6 \mathrm{mg}$ of $\mathrm{N}$-(p-tolyl)hydroxylamine (1d), 3d was isolated as yellow oil $(48.1 \mathrm{mg}$, a mixture of geometric isomers (94:6), 95\%). ${ }^{1} \mathrm{H} \mathrm{NMR}\left(300 \mathrm{MHz}, \mathrm{CDCl}_{3}\right)$ major isomer $\delta$ 7.89-7.83 (m, 2H), 7.52-7.43 (m, 3H), $7.13(\mathrm{~d}, J=6.1 \mathrm{~Hz}, 2 \mathrm{H}), 6.88(\mathrm{~d}, J=6.1 \mathrm{~Hz}$, 2H), $3.66(\mathrm{~s}, 3 \mathrm{H}), 2.33(\mathrm{~s}, 3 \mathrm{H}) .{ }^{13} \mathrm{C} \mathrm{NMR}\left(75 \mathrm{MHz}, \mathrm{CDCl}_{3}\right) \delta 165.9,159.6,147.5$, $134.8,134.0,131.7,129.6,128.8,127.9,119.6,52.0,21.0$.<smiles>COC(=O)/C(=N/c1ccc(OC)cc1)c1ccccc1</smiles>

\section{Methyl 2-((4-methoxyphenyl)imino)-2-phenylacetate $(3 e)^{4}$}

The title compound was prepared via general procedure using $27.8 \mathrm{mg}$ of $\mathrm{N}-(4-m e t h o x y p h e n y l) h y d r o x y l a m i n e ~(1 e), 3 e$ was isolated as a yellow solid (50.6 mg, a mixture of geometric isomers (92:8), 94\%), mp: 89-90 ${ }^{\circ} \mathrm{C} .{ }^{1} \mathrm{H}$ NMR (400 MHz, $\mathrm{CDCl}_{3}$ ) major isomer $\delta$ 7.90-7.78 $(\mathrm{m}, 2 \mathrm{H}), 7.54-7.41(\mathrm{~m}, 3 \mathrm{H}), 7.00-6.93(\mathrm{~m}, 2 \mathrm{H})$, 6.92-6.84 (m, 2H), $3.81(\mathrm{~s}, 3 \mathrm{H}), 3.69(\mathrm{~s}, 3 \mathrm{H}) .{ }^{13} \mathrm{C} \mathrm{NMR}\left(100 \mathrm{MHz}, \mathrm{CDCl}_{3}\right) \delta 166.1$, $159.2,157.4,143.2,134.1,131.6,128.7,127.8,121.2,114.2,55.4,52.0$. 
<smiles>CCOC(=O)c1ccc(/N=C(\OCC)c2ccccc2)cc1</smiles>

\section{Ethyl 4-((2-methoxy-2-oxo-1-phenylethylidene)amino)benzoate (3f)}

The title compound was prepared via general procedure using $36.2 \mathrm{mg}$ of N-(4-ethoxycarbonylphenyl)hydroxylamine (1f), 3f was isolated as yellow oil (56.6 mg, a mixture of geometric isomers (95:5), 91\%). ${ }^{1} \mathrm{H}$ NMR (400 $\left.\mathrm{MHz}, \mathrm{CDCl}_{3}\right)$ major isomer $\delta 8.04(\mathrm{~d}, J=8.5 \mathrm{~Hz}, 2 \mathrm{H}), 7.90-7.84(\mathrm{~m}, 2 \mathrm{H}), 7.58-7.52(\mathrm{~m}, 1 \mathrm{H}), 7.52-7.44(\mathrm{~m}$, 2H), $6.98(\mathrm{~d}, J=8.5 \mathrm{~Hz}, 2 \mathrm{H}), 4.38(\mathrm{q}, J=7.1 \mathrm{~Hz}, 2 \mathrm{H}), 3.63(\mathrm{~s}, 3 \mathrm{H}), 1.40$ (t, $J=7.1$ $\mathrm{Hz}, 3 \mathrm{H}) .{ }^{13} \mathrm{C}$ NMR $\left(100 \mathrm{MHz}, \mathrm{CDCl}_{3}\right) \delta 166.3,164.8,160.5,154.2,133.3,132.3$, 130.6, 128.9, 128.2, 126.9, 119.3, 60.9, 52.1, 14.4. HRMS (ESI) calculated for $\mathrm{C}_{18} \mathrm{H}_{18} \mathrm{NO}_{4}[\mathrm{M}+\mathrm{H}]^{+}: 312.1230$, found: 312.1233 .<smiles>COC(=O)/C(=N/c1ccc(Cl)cc1)c1ccccc1</smiles>

\section{Methyl 2-((4-chlorophenyl)imino)-2-phenylacetate (3g)}

The title compound was prepared via general procedure using $28.7 \mathrm{mg}$ of

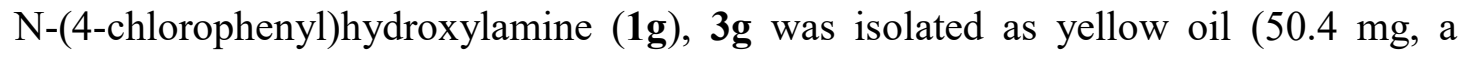
mixture of geometric isomers (95:5), 92\%). ${ }^{1} \mathrm{H}$ NMR $\left(300 \mathrm{MHz}, \mathrm{CDCl}_{3}\right)$ major isomer $\delta$ 7.88-7.82 (m, 2H), 7.55-7.50 (m, 1H), 7.49-7.43 (m, 2H), 7.32-7.27 (m, 2H), 6.93-6.87 (m, 2H), 3.67 (s, 3H). ${ }^{13} \mathrm{C} \mathrm{NMR}\left(75 \mathrm{MHz}, \mathrm{CDCl}_{3}\right) \delta 165.2,160.6,148.6$, 133.5, 132.1, 130.4, 129.1, 128.9, 128.1, 121.0, 52.1. HRMS (ESI) calculated for $\mathrm{C}_{15} \mathrm{H}_{13} \mathrm{ClNO}_{2}[\mathrm{M}+\mathrm{H}]^{+}:$274.0629, found: 274.0624.

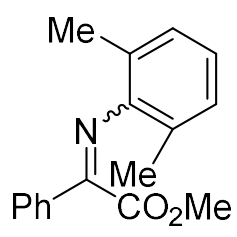




\section{Methyl 2-((2,6-dimethylphenyl)imino)-2-phenylacetate (3h)}

The title compound was prepared via general procedure using $27.4 \mathrm{mg}$ of N-(2,6-dimethylphenyl)hydroxylamine (1h), $\mathbf{3 h}$ was isolated as yellow oil (45.4 mg, a mixture of geometric isomers (94:6), 85\%). ${ }^{1} \mathrm{H}$ NMR (400 $\left.\mathrm{MHz}, \mathrm{CDCl}_{3}\right)$ major isomer $\delta 7.92(\mathrm{~d}, J=7.4 \mathrm{~Hz}, 2 \mathrm{H}), 7.57-7.45(\mathrm{~m}, 3 \mathrm{H}), 7.0(\mathrm{~d}, J=7.5 \mathrm{~Hz}, 2 \mathrm{H}), 6.90(\mathrm{t}$, $J=7.5 \mathrm{~Hz}, 1 \mathrm{H}), 3.55(\mathrm{~s}, 3 \mathrm{H}), 2.10(\mathrm{~s}, 6 \mathrm{H}) \cdot{ }^{13} \mathrm{C} \mathrm{NMR}\left(100 \mathrm{MHz}, \mathrm{CDCl}_{3}\right) \delta 165.3$, $160.8,148.0,133.4,131.9,128.8,127.9,127.6,126.3,123.8,51.9,18.1$. HRMS (ESI) calculated for $\mathrm{C}_{17} \mathrm{H}_{18} \mathrm{NO}_{2}[\mathrm{M}+\mathrm{H}]^{+}:$268.1132, found: 268.1136 .<smiles>CC(=O)/C(=N\c1ccccc1)c1ccc(C)cc1</smiles>

\section{Methyl 2-(phenylimino)-2-(p-tolyl)acetate (3i)}

The title compound was prepared via general procedure using $21.8 \mathrm{mg}$ of N-phenylhydroxylamine (1a), 3i was isolated as yellow oil (47.6 mg, a mixture of geometric isomers (95:5), 94\%). ${ }^{1} \mathrm{H}$ NMR (300 $\left.\mathrm{MHz}, \mathrm{CDCl}_{3}\right)$ major isomer $\delta$ 7.79-7.73 (m, 2H), 7.36-7.20 (m, 2H), 7.29-7.24 (m, 2H), 7.16-7.09 (m, 1H), 6.98-6.92 (m, 2H), $3.62(\mathrm{~s}, 3 \mathrm{H}), 2.41(\mathrm{~s}, 3 \mathrm{H}) .{ }^{13} \mathrm{C} \mathrm{NMR}\left(100 \mathrm{MHz}, \mathrm{CDCl}_{3}\right) \delta$ 165.6, 159.9, 150.2, 142.5, 131.2, 129.5, 128.9, 128.0, 124.9, 119.6, 51.9, 21.6. HRMS (ESI) calculated for $\mathrm{C}_{16} \mathrm{H}_{16} \mathrm{NO}_{2}[\mathrm{M}+\mathrm{H}]^{+}:$254.1176, found: 254.1173 .<smiles>COC(=O)/C(=N\c1ccccc1)c1cccc(OC)c1</smiles>

\section{Methyl 2-(3-methoxyphenyl)-2-(phenylimino)acetate (3j)}

The title compound was prepared via general procedure using $21.8 \mathrm{mg}$ of $\mathrm{N}$-phenylhydroxylamine (1a), 3j was isolated as yellow oil $(50.6 \mathrm{mg}$, a mixture of geometric isomers (94:6), 94\%). ${ }^{1} \mathrm{H}$ NMR (400 $\left.\mathrm{MHz}, \mathrm{CDCl}_{3}\right)$ major isomer $\delta$ 7.54-7.49 (m, 1H), 7.39-7.28 (m, 4H), 7.13 (t, $J=7.4 \mathrm{~Hz}, 1 \mathrm{H}), 7.09-7.02(\mathrm{~m}, 1 \mathrm{H})$, 7.00-6.92 (m, 2H), 3.85 (s, 3H), $3.61(\mathrm{~s}, 3 \mathrm{H}) .{ }^{13} \mathrm{C} \mathrm{NMR}\left(100 \mathrm{MHz}, \mathrm{CDCl}_{3}\right) \delta 165.4$, 
160.0, 159.9, 150.0, 135.1, 129.8, 129.0, 125.1, 121.0, 119.5, 118.6, 111.9, 55.5, 52.0.

HRMS (ESI) calculated for $\mathrm{C}_{16} \mathrm{H}_{16} \mathrm{NO}_{3}[\mathrm{M}+\mathrm{H}]^{+}: 270.1125$, found: 270.1129 .<smiles>CC(=O)/C(=N\c1ccccc1)c1ccc(C(F)(F)F)cc1</smiles>

\section{Methyl 2-(phenylimino)-2-(4-(trifluoromethyl)phenyl)acetate (3k)}

The title compound was prepared via general procedure using $21.8 \mathrm{mg}$ of $\mathrm{N}$-phenylhydroxylamine (1a), 3k was isolated as yellow oil $(57.1 \mathrm{mg}$, a mixture of geometric isomers (93:7), 93\%). ${ }^{1} \mathrm{H}$ NMR $\left(300 \mathrm{MHz}, \mathrm{CDCl}_{3}\right)$ major isomer $\delta 8.00(\mathrm{~d}$, $J=8.2 \mathrm{~Hz}, 2 \mathrm{H}), 7.73(\mathrm{~d}, J=8.2 \mathrm{~Hz}, 2 \mathrm{H}), 7.39-7.32(\mathrm{~m}, 2 \mathrm{H}), 7.18(\mathrm{t}, J=7.4 \mathrm{~Hz}, 1 \mathrm{H})$, 7.00-6.92 (m, 2H), $3.65(\mathrm{~s}, 3 \mathrm{H}) .{ }^{13} \mathrm{C} \mathrm{NMR}\left(100 \mathrm{MHz}, \mathrm{CDCl}_{3}\right) \delta$ 165.0, 158.5, 149.6, 137.0, 129.0, 128.4, 125.73, 125.69, 125.5, 125.2, 125.1, 119.5, 119.4, 52.2. HRMS (ESI) calculated for $\mathrm{C}_{16} \mathrm{H}_{13} \mathrm{~F}_{3} \mathrm{NO}_{2}[\mathrm{M}+\mathrm{H}]^{+}:$308.0893, found: 308.0896 .

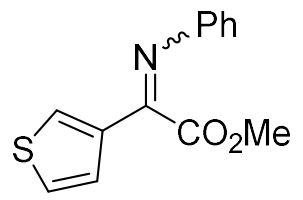

\section{Methyl 2-(phenylimino)-2-(thiophen-3-yl)acetate (3l)}

The title compound was prepared via general procedure using $21.8 \mathrm{mg}$ of N-phenylhydroxylamine (1a), 3l was isolated as yellow oil (46.6 mg, a mixture of geometric isomers (89:11), 95\%). ${ }^{1} \mathrm{H}$ NMR $\left(400 \mathrm{MHz}, \mathrm{CDCl}_{3}\right)$ major isomer $\delta$ 7.85-7.76 (m, 1H), 7.69-7.63 (m, 1H), 7.39-7.35 (m, 1H), 7.35-7.30 (m, 2H), 7.16-7.10 (m, 1H), 6.97-6.91 (m, 2H), $\left.3.60(\mathrm{~s}, 3 \mathrm{H}) .{ }^{13} \mathrm{C} \mathrm{NMR} \mathrm{(100} \mathrm{MHz,} \mathrm{CDCl}_{3}\right) \delta$ $165.3,154.8,150.0,137.7,129.9,129.0,126.7,126.5,125.0,119.6,52.1$. HRMS (ESI) calculated for $\mathrm{C}_{13} \mathrm{H}_{12} \mathrm{NO}_{2} \mathrm{~S}[\mathrm{M}+\mathrm{H}]^{+}:$246.0583, found: 246.0587 .<smiles>COC(=O)C(=Nc1ccccc1)C(=O)OC</smiles> 


\section{Dimethyl 2-(phenylimino)malonate $(3 m)^{6}$}

The title compound was prepared at $80{ }^{\circ} \mathrm{C}$ for $6 \mathrm{~h}$ via general procedure using 21.8 mg of N-phenylhydroxylamine (1a), 3m was isolated as yellow oil (30.9 mg, 70\%). ${ }^{1} \mathrm{H}$ NMR $\left(400 \mathrm{MHz}, \mathrm{CDCl}_{3}\right) \delta$ 7.40-7.33 (m, 2H), 7.26-7.21 (m, 1H), 7.05-6.96 (m, 2H), 3.99 (s, 3H), 3.70 (s, 3H). ${ }^{13} \mathrm{C} \mathrm{NMR}\left(100 \mathrm{MHz}, \mathrm{CDCl}_{3}\right) \delta$ 162.9, 161.5, 147.3, $129.1,127.2,119.7,115.6,53.7,52.7$.

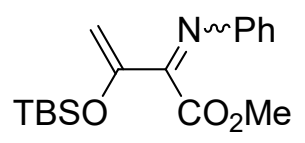

\section{Methyl 3-((tert-butyldimethylsilyl)oxy)-2-(phenylimino)but-3-enoate (3n)}

The title compound was prepared via general procedure using $21.8 \mathrm{mg}$ of N-phenylhydroxylamine (1a), 3n was isolated as yellow oil (44.7 mg, a mixture of geometric isomers (95:5), 70\%). ${ }^{1} \mathrm{H}$ NMR (400 $\left.\mathrm{MHz}, \mathrm{CDCl}_{3}\right)$ major isomer $\delta$ 7.33-7.27 (m, 2H), 7.14-7.08 (m, 1H), 6.92-6.82 (m, 2H), 5.12 (d, J=2.0 Hz, 1H), $4.95(\mathrm{~d}, J=2.0 \mathrm{~Hz}, 1 \mathrm{H}), 3.56$ (s, 3H), 0.96 (s, 9H), 0.24 (s, 6H). ${ }^{13} \mathrm{C}$ NMR (100 MHz, $\left.\mathrm{CDCl}_{3}\right) \delta 164.9,158.4,152.1,149.3,128.8,125.1,119.4,102.4,51.9,25.6,18.4,-4.6$. HRMS (ESI) calculated for $\mathrm{C}_{17} \mathrm{H}_{26} \mathrm{NO}_{3} \mathrm{Si}[\mathrm{M}+\mathrm{H}]^{+}: 320.1676$, found: 320.1273 .<smiles>FC(F)(F)/C(=N\c1ccccc1)c1ccccc1</smiles>

\section{2,2,2-trifluoro-N,1-diphenylethan-1-imine (3o $)^{7}$}

The title compound was prepared via general procedure using $21.8 \mathrm{mg}$ of N-phenylhydroxylamine (1a), 3o was isolated as yellow oil (47.9 mg, a mixture of geometric isomers (97:3), 96\%). ${ }^{1} \mathrm{H} \mathrm{NMR}\left(400 \mathrm{MHz}, \mathrm{CDCl}_{3}\right)$ major isomer $\delta$ 7.36-7.31 (m, 1H), 7.31-7.25 (m, 2H), 7.24-7.15 (m, 4H), 7.03 (t, J=7.4 Hz, 1H), 6.74 $(\mathrm{d}, J=7.4 \mathrm{~Hz}, 2 \mathrm{H}) .{ }^{13} \mathrm{C} \mathrm{NMR}\left(100 \mathrm{MHz}, \mathrm{CDCl}_{3}\right) \delta 157.3,156.9,147.1,130.3,130.0$, $128.8,128.7,128.6,125.4,120.6,118.5$. 
<smiles>COP(=O)(OC)/C(=N\c1ccccc1)c1ccccc1</smiles>

\section{Dimethyl(phenyl(phenylimino)methyl)phosphonate $(3 p)^{8}$}

The title compound was prepared $80{ }^{\circ} \mathrm{C}$ for $1 \mathrm{~h}$ via general procedure using $21.8 \mathrm{mg}$ of N-phenylhydroxylamine (1a), 3p was isolated as yellow oil (46.3 mg, a mixture of geometric isomers (90:10), 80\%). ${ }^{1} \mathrm{H}$ NMR (400 $\left.\mathrm{MHz}, \mathrm{CDCl}_{3}\right)$ major isomer $\delta$ 7.33-7.22 (m, 5H), $7.17(\mathrm{t}, J=7.8 \mathrm{~Hz}, 2 \mathrm{H}), 7.01(\mathrm{t}, J=7.4 \mathrm{~Hz}, 1 \mathrm{H}), 6.73(\mathrm{~d}, J=7.8$ $\mathrm{Hz}, 2 \mathrm{H}), 3.91$ (s, 3H), $3.88(\mathrm{~s}, 3 \mathrm{H}) .{ }^{13} \mathrm{C} \mathrm{NMR}\left(100 \mathrm{MHz}, \mathrm{CDCl}_{3}\right) \delta$ 167.9, 165.7, $149.1,148.8,133.8,133.4,133.1,130.0,129.6,128.7,128.6,128.5,128.39,128.35$, $125.0,120.7,120.7,54.3,54.2$.<smiles>C/C(=N\c1ccccc1)c1ccccc1</smiles>

\section{N,1-diphenylethan-1-imine (3q)}

The title compound was prepared via general procedure using $21.8 \mathrm{mg}$ of N-phenylhydroxylamine (1a), 3q was isolated as yellow oil $(24.1 \mathrm{mg}$, a mixture of geometric isomers $(87: 13), 62 \%) .{ }^{1} \mathrm{H}$ NMR (400 MHz, DMSO-d $\left.\mathrm{d}_{6}\right)$ major isomer $\delta$ 8.02-7.96 (m, 2H), 7.53-7.44 (m, 3H), 7.37 (t, $J=7.8 \mathrm{~Hz}, 2 \mathrm{H}), 7.08$ (t, $J=7.5 \mathrm{~Hz}$, $1 \mathrm{H}), 6.79(\mathrm{~d}, J=7.5 \mathrm{~Hz}, 2 \mathrm{H}), 2.20(\mathrm{~s}, 3 \mathrm{H}) .{ }^{13} \mathrm{C}$ NMR (75 MHz, DMSO-d 6$) \delta 165.4$, $151.8,139.3,131.0,129.5,128.8,127.6,123.6,119.7,17.6$.<smiles>C/C(=N\c1ccccc1)c1ccc(Br)cc1</smiles>

\section{1-(4-bromophenyl)-N-phenylethan-1-imine $(3 r)^{10}$}

The title compound was prepared via general procedure using $21.8 \mathrm{mg}$ of N-phenylhydroxylamine (1a), 3r was isolated as a yellow solid $(30.1 \mathrm{mg}$, a mixture of 
geometric isomers (93:7), 55\%). ${ }^{1} \mathrm{H}$ NMR (300 MHz, DMSO- $\mathrm{d}_{6}$ ) major isomer $\delta$ 7.96-7.90 (m, 2H), 7.71-7.66 (m, 2H), 7.42-7.33 (m, 2H), 7.14-7.05 (m, 1H), 6.84-6.76 (m, 2H), 2.19 (s, 3H). ${ }^{13} \mathrm{C}$ NMR (75 MHz, DMSO-d 6 ) $\delta 164.7,151.5,138.5$, $131.8,129.7,129.5,124.8,123.8,119.7,17.5$.

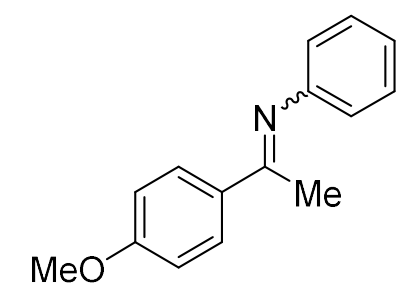

\section{1-(4-methoxyphenyl)-N-phenylethan-1-imine $(3 s)^{11}$}

The title compound was prepared via general procedure using $21.8 \mathrm{mg}$ of N-phenylhydroxylamine (1a), 3s was isolated as a white solid (31.5 mg, a mixture of geometric isomers (91:9), 70\%). ${ }^{1} \mathrm{H}$ NMR (300 MHz, DMSO-d 6 ) major isomer $\delta$ 7.99-7.90 (m, 2H), 7.39-7.31 (m, 2H), 7.10-6.99 (m, 3H), 6.80-6.72 (m, 2H), 3.82 (s, $3 \mathrm{H}), 2.16$ (s, 3H). ${ }^{13} \mathrm{C}$ NMR (75 MHz, DMSO-d 6 ) $\delta$ 164.4, 161.7, 152.0, 131.9, 131.0, 129.4, 129.31, 129.26, 123.3, 119.9, 116.1, 114.33, 114.30, 114.1, 56.0, 55.8, 26.9, 17.3 .

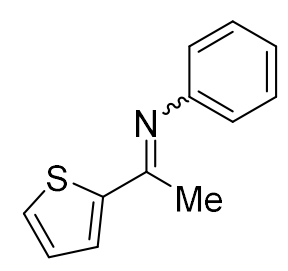

\section{$N$-phenyl-1-(thiophen-2-yl)ethan-1-imine (3t) ${ }^{12}$}

The title compound was prepared via general procedure using $21.8 \mathrm{mg}$ of $\mathrm{N}$-phenylhydroxylamine (1a), 3t was isolated as a yellow solid $(26.1 \mathrm{mg}, 65 \%) .{ }^{1} \mathrm{H}$ NMR (400 MHz, DMSO-d 6 ) $\delta 7.73$ (d, $J=5.0 \mathrm{~Hz}, 1 \mathrm{H}), 7.66$ (d, $J=3.1 \mathrm{~Hz}, 1 \mathrm{H}), 7.36$ (t, $J=7.8 \mathrm{~Hz}, 2 \mathrm{H}), 7.17(\mathrm{t}, J=3.1 \mathrm{~Hz}, 1 \mathrm{H}), 7.09$ (t, $J=7.8 \mathrm{~Hz}, 1 \mathrm{H}), 6.81$ (d, $J=7.8$ $\mathrm{Hz}, 2 \mathrm{H}), 2.21$ (s, 3H). ${ }^{13} \mathrm{C}$ NMR (75 MHz, DMSO-d 6 ) $8160.8,150.6,146.3,131.2$, 130.3, 129.4, 128.4, 123.9, 120.3, 17.6. 


\section{General procedure for Scheme 4}

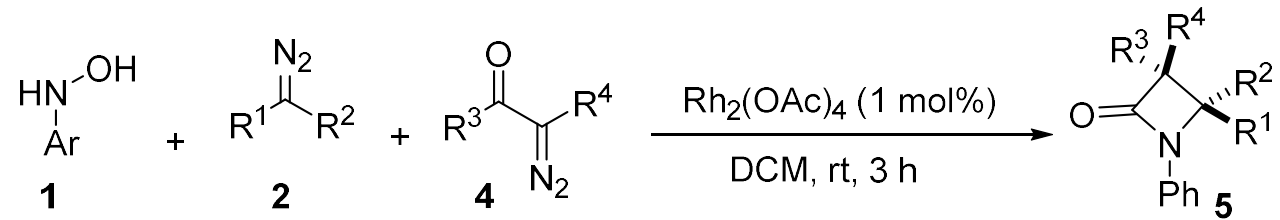

To a tube was added $\mathrm{Rh}_{2}(\mathrm{OAc})_{4}(0.002 \mathrm{mmol}, 0.01 \mathrm{eq}), 1(0.2 \mathrm{mmol}, 1 \mathrm{eq}), 2(0.24$ mmol, $1.2 \mathrm{eq}), 4(0.36 \mathrm{mmol}, 1.8 \mathrm{eq})$ and DCM (4 mL) under argon atmosphere, the resulting solution stirred at $\mathrm{rt}$ for $3 \mathrm{~h}$, [Note: $\mathbf{5 o}, \mathbf{5 p}$ and $\mathbf{5 r}$ were performed at $80{ }^{\circ} \mathrm{C}$ ]. The reaction mixture was concentrated under vacuum, the residue was purified by column chromatography (silica gel, eluted with EtOAc: Petroleum ether $=1: 10$ ) to give product 5 .<smiles>COC(=O)C1(C(C)=O)C(=O)N(c2ccccc2)C1(C(C)=O)c1ccccc1</smiles>

Dimethyl4-oxo-1,2-diphenyl-3-((E)-styryl)azetidine-2,3-dicarboxylate (5a)

The title compound was prepared via general procedure using $21.8 \mathrm{mg}$ of $\mathrm{N}$-phenylhydroxylamine (1a), 5a was isolated as a white solid (73.3 $\mathrm{mg}, 83 \%), \mathrm{mp}$ : 170-172 ${ }^{\circ} \mathrm{C} .{ }^{1} \mathrm{H}$ NMR $\left(400 \mathrm{MHz}, \mathrm{CDCl}_{3}\right) \delta$ 7.76-7.68 (m, 2H), 7.49-7.41 (m, 4H), 7.37-7.26 (m, 8H), 7.13 (t, $J=7.4 \mathrm{~Hz}, 1 \mathrm{H}), 6.97$ (d, $J=16.4 \mathrm{~Hz}, 1 \mathrm{H}), 6.56$ (d, $J=$ 16.4 Hz, 1H), 3.60 (s, 3H), 3.17 (s, 3H). ${ }^{13} \mathrm{C}$ NMR (100 MHz, CDCl3) $\delta$ 167.8, 166.3, 161.0, 137.2, 135.9, 135.2, 131.0, 129.1, 128.79, 128.75, 128.6, 128.3, 128.1, 126.9, 124.6, 118.9, 118.5, 76.3, 75.0, 53.0, 52.4. HRMS (ESI) calculated for $\mathrm{C}_{27} \mathrm{H}_{24} \mathrm{NO}_{5}$ $[\mathrm{M}+\mathrm{H}]^{+}:$442.1649, found: 442.1644 .

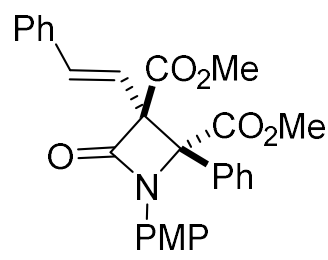


Dimethyl1-(4-methoxyphenyl)-4-oxo-2-phenyl-3-((E)-styryl)azetidine-2,3-dicarboxy late (5b)

The title compound was prepared via general procedure using $27.8 \mathrm{mg}$ of N-(4-methoxyphenyl)hydroxylamine (1e), 5b was isolated as a white solid (77.3 $\mathrm{mg}$, 82\%), mp: $174-176{ }^{\circ} \mathrm{C} .{ }^{1} \mathrm{H}$ NMR (400 $\left.\mathrm{MHz}, \mathrm{CDCl}_{3}\right) \delta$ 7.75-7.69 (m, 2H), 7.45-7.39 (m, 4H), 7.35-7.26 (m, 6H), $6.97(\mathrm{~d}, J=16.4 \mathrm{~Hz}, 1 \mathrm{H}), 6.84(\mathrm{~d}, J=9.0 \mathrm{~Hz}, 2 \mathrm{H}), 6.56$ $(\mathrm{d}, J=16.4 \mathrm{~Hz}, 1 \mathrm{H}), 3.78$ (s, 3H), 3.61 (s, 3H), 3.17 (s, 3H). ${ }^{13} \mathrm{C}$ NMR (100 MHz, $\left.\mathrm{CDCl}_{3}\right) \delta 167.9,166.4,160.5,156.4,135.9,135.1,131.1,130.7,129.1,128.7,128.6$, 128.3, 128.1, 126.9, 119.8, 119.1, 114.0, 76.3, 75.1, 55.5, 53.0, 52.3. HRMS (ESI) calculated for $\mathrm{C}_{28} \mathrm{H}_{26} \mathrm{NO}_{6}[\mathrm{M}+\mathrm{H}]^{+}:$472.1755, found: 472.1751 .

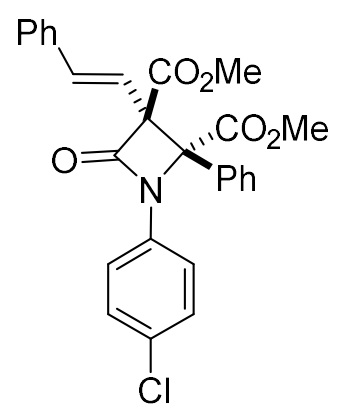

Dimethyl1-(4-chlorophenyl)-4-oxo-2-phenyl-3-((E)-styryl)azetidine-2,3-dicarboxylat $e(5 c)$

The title compound was prepared via general procedure using $28.7 \mathrm{mg}$ of N-(4-chlorophenyl)hydroxylamine (1g), 5c was isolated as a white solid (76.1 $\mathrm{mg}$, 80\%), mp: $122-123{ }^{\circ} \mathrm{C} .{ }^{1} \mathrm{H}$ NMR (400 MHz, $\left.\mathrm{CDCl}_{3}\right) \delta$ 7.72-7.66 (m, 2H), 7.45-7.40 (m, 4H), 7.38-7.31 (m, 5H), 7.31-7.25 (m, 3H), $6.95(\mathrm{~d}, J=16.4 \mathrm{~Hz}, 1 \mathrm{H}), 6.55(\mathrm{~d}, J=$ $16.4 \mathrm{~Hz}, 1 \mathrm{H}), 3.62(\mathrm{~s}, 3 \mathrm{H}), 3.18(\mathrm{~s}, 3 \mathrm{H}) .{ }^{13} \mathrm{C} \mathrm{NMR}\left(100 \mathrm{MHz}, \mathrm{CDCl}_{3}\right) \delta$ 167.5, 166.0, $160.9,135.73,135.69,135.2,130.6,129.8,129.3,128.9,128.8,128.7,128.3,128.1$, 126.9, 119.8, 118.8, 76.6, 75.3, 53.1, 52.4. HRMS (ESI) calculated for $\mathrm{C}_{27} \mathrm{H}_{23} \mathrm{ClNO}_{5}$ $[\mathrm{M}+\mathrm{H}]^{+}:$476.1259, found: 476.1255 . 


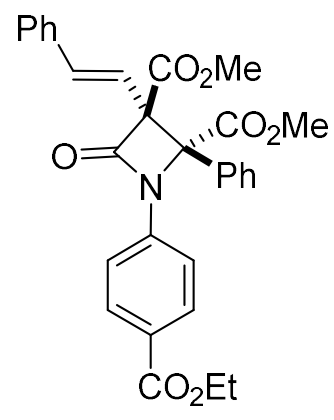

Dimethyl1-(4-(ethoxycarbonyl)phenyl)-4-oxo-2-phenyl-3-((E)-styryl)azetidine-2,3-di carboxylate (5d)

The title compound was prepared via general procedure using $36.2 \mathrm{mg}$ of N-(4-ethoxycarbonylphenyl)hydroxylamine (1f), 5d was isolated as a white solid (84.2 mg, 82\%), mp: 145-146 ${ }^{\circ} \mathrm{C} .{ }^{1} \mathrm{H}$ NMR (400 MHz, $\left.\mathrm{CDCl}_{3}\right) \delta 8.01$ (d, J=8.8 Hz, 2H), 7.75-7.66 (m, 2H), 7.51 (d, $J=8.8 \mathrm{~Hz}, 2 \mathrm{H}), 7.46-7.41(\mathrm{~m}, 2 \mathrm{H}), 7.39-7.26(\mathrm{~m}$, $6 \mathrm{H}), 6.96(\mathrm{~d}, J=16.4 \mathrm{~Hz}, 1 \mathrm{H}), 6.56(\mathrm{~d}, J=16.4 \mathrm{~Hz}, 1 \mathrm{H}), 4.40-4.32(\mathrm{~m}, 2 \mathrm{H}), 3.61$ (s, $3 \mathrm{H}), 3.19(\mathrm{~s}, 3 \mathrm{H}), 1.38(\mathrm{t}, J=7.1 \mathrm{~Hz}, 3 \mathrm{H}) \cdot{ }^{13} \mathrm{C} \mathrm{NMR}\left(100 \mathrm{MHz}, \mathrm{CDCl}_{3}\right) \delta 167.4$, $166.0,165.9,161.3,140.7,135.7,135.3,130.49,130.46,129.3,128.8,128.3,128.1$, 126.9, 126.4, 118.7, 118.1, 76.6, 75.3, 61.0, 53.2, 52.5, 14.4. HRMS (ESI) calculated for $\mathrm{C}_{30} \mathrm{H}_{28} \mathrm{NO}_{7}[\mathrm{M}+\mathrm{H}]^{+}:$514.1860, found: 514.1864 .

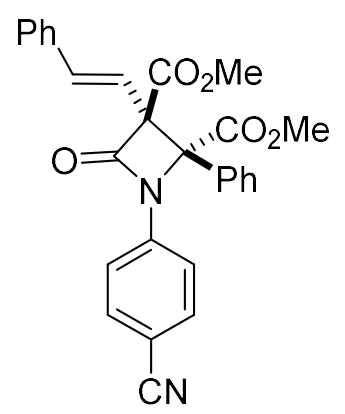

Dimethyl1-(4-cyanophenyl)-4-oxo-2-phenyl-3-((E)-styryl)azetidine-2,3-dicarboxylat e (5e)

The title compound was prepared via general procedure using $26.8 \mathrm{mg}$ of N-(4-cyanophenyl)hydroxylamine (1i), 5e was isolated as a white solid (81.1 $\mathrm{mg}$, 87\%), mp: $164-165{ }^{\circ} \mathrm{C} .{ }^{1} \mathrm{H}$ NMR (400 MHz, $\left.\mathrm{CDCl}_{3}\right) \delta$ 7.70-7.64 (m, 2H), 7.63-7.53 (m, 4H), 7.45-7.41 (m, 2H), 7.41-7.29 (m, 6H), $6.92(\mathrm{~d}, J=16.4 \mathrm{~Hz}, 1 \mathrm{H}), 6.55(\mathrm{~d}, J=$ $16.4 \mathrm{~Hz}, 1 \mathrm{H}), 3.65$ (s, 3H), $3.20(\mathrm{~s}, 3 \mathrm{H}) .{ }^{13} \mathrm{C} \mathrm{NMR}\left(100 \mathrm{MHz}, \mathrm{CDCl}_{3}\right) \delta 167.1,165.7$, 
$161.4,140.6,135.5,135.3,133.0,130.1,129.6,128.9,128.8,128.5,127.9,126.9$, 118.9, 118.6, 118.5, 107.8, 76.9, 75.7, 53.3, 52.5. HRMS (ESI) calculated for $\mathrm{C}_{28} \mathrm{H}_{23} \mathrm{~N}_{2} \mathrm{O}_{5}[\mathrm{M}+\mathrm{H}]^{+}:$467.1601, found: 467.1603 .

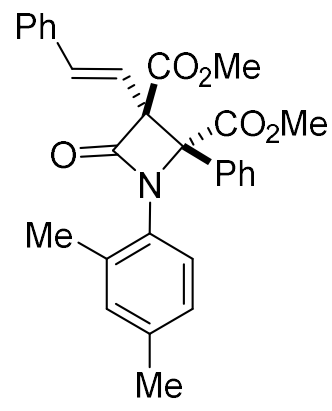

Dimethyl1-(2,4-dimethylphenyl)-4-oxo-2-phenyl-3-((E)-styryl)azetidine-2,3-dicarbo xylate (5f)

The title compound was prepared via general procedure using $27.4 \mathrm{mg}$ of $\mathrm{N}-(2,4-d i m e t h y l p h e n y l) h y d r o x y l a m i n e ~(\mathbf{1 j}), \mathbf{5 f}$ was isolated as colorless oil (73.2 $\mathrm{mg}$, 78\%). ${ }^{1} \mathrm{H}$ NMR $\left(400 \mathrm{MHz}, \mathrm{CDCl}_{3}\right) \delta$ 7.49-7.43 (m, 4H), 7.37-7.26 (m, 6H), $7.15(\mathrm{~s}$, 1H), $7.12(\mathrm{~d}, J=8.2 \mathrm{~Hz}, 1 \mathrm{H}), 7.06(\mathrm{~d}, J=16.4 \mathrm{~Hz}, 1 \mathrm{H}), 6.90(\mathrm{~d}, J=8.2 \mathrm{~Hz}, 1 \mathrm{H}), 6.60$ $(\mathrm{d}, J=16.4 \mathrm{~Hz}, 1 \mathrm{H}), 3.67(\mathrm{~s}, 3 \mathrm{H}), 3.27$ (s, 3H), 2.49 (s, 3H), $2.32(\mathrm{~s}, 3 \mathrm{H}) .{ }^{13} \mathrm{C} \mathrm{NMR}$ $\left(100 \mathrm{MHz} \mathrm{CDCl}_{3}\right) \delta 168.9,167.1,161.2,137.5,136.1,135.8,134.8,133.4,132.6$, 132.5, 128.8, 128.7, 128.6, 128.0, 127.8, 127.1, 126.9, 123.1, 119.0, 77.3, 75.3, 53.0, 52.4, 21.0, 19.7. HRMS (ESI) calculated for $\mathrm{C}_{29} \mathrm{H}_{28} \mathrm{NO}_{5}[\mathrm{M}+\mathrm{H}]^{+}: 470.1962$, found: 470.1965 .<smiles>COC(=O)C1(C(C)=O)C(=O)N(c2ccccc2C)C1(C(C)=O)c1ccccc1</smiles>

Dimethyl4-oxo-2-phenyl-3-((E)-styryl)-1-(o-tolyl)azetidine-2,3-dicarboxylate (5g)

The title compound was prepared via general procedure using $24.6 \mathrm{mg}$ of $\mathrm{N}$-(o-tolyl)hydroxylamine (1b), 5g was isolated as colorless oil $(65.6 \mathrm{mg}, 72 \%) .{ }^{1} \mathrm{H}$

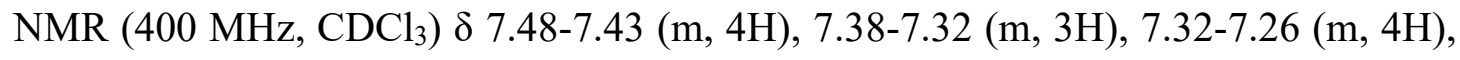


7.25-7.18 (m, 2H), 7.13-7.04 (m, 2H), $6.60(\mathrm{~d}, J=16.4 \mathrm{~Hz}, 1 \mathrm{H}), 3.68(\mathrm{~s}, 3 \mathrm{H}), 3.27(\mathrm{~s}$, 3H), $2.54(\mathrm{~s}, 3 \mathrm{H}) .{ }^{13} \mathrm{C} \mathrm{NMR}\left(100 \mathrm{MHz}, \mathrm{CDCl}_{3}\right) \delta 168.8,167.0,161.1,136.0,135.9$, $135.2,134.9,133.2,132.0,128.79,128.77,128.6,128.0,127.8,127.6,126.9,126.4$, 123.0, 118.9, 77.2, 75.3, 53.1, 52.4, 19.9. HRMS (ESI) calculated for $\mathrm{C}_{28} \mathrm{H}_{26} \mathrm{NO}_{5}$ $[\mathrm{M}+\mathrm{H}]^{+}:$456.1805, found: 456.1809 .

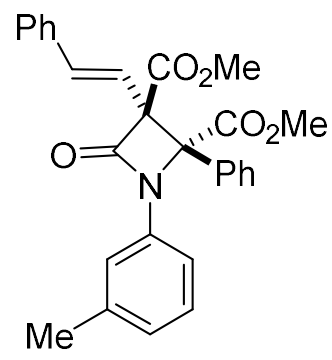

\section{Dimethyl4-oxo-2-phenyl-3-((E)-styryl)-1-(m-tolyl)azetidine-2,3-dicarboxylate (5h)}

The title compound was prepared via general procedure using $24.6 \mathrm{mg}$ of $\mathrm{N}$-( $m$-tolyl)hydroxylamine (1c), $\mathbf{5 h}$ was isolated as a white solid (76.5 $\mathrm{mg}, 84 \%), \mathrm{mp}$ : 142-144 ${ }^{\circ} \mathrm{C} .{ }^{1} \mathrm{H}$ NMR $\left(400 \mathrm{MHz}, \mathrm{CDCl}_{3}\right) \delta$ 7.76-7.69 (m, 2H), 7.56 (s, 1H), 7.46-7.40 (m, 2H), 7.37-7.30 (m, 5H), 7.29-7.24 (m, 1H), 7.15 (t, $J=7.8 \mathrm{~Hz}, 1 \mathrm{H}), 7.02-6.92(\mathrm{~m}$, $3 \mathrm{H}), 6.56(\mathrm{~d}, J=16.4 \mathrm{~Hz}, 1 \mathrm{H}), 3.59(\mathrm{~s}, 3 \mathrm{H}), 3.16(\mathrm{~s}, 3 \mathrm{H}), 2.33(\mathrm{~s}, 3 \mathrm{H}) .{ }^{13} \mathrm{C}$ NMR $(100$ $\left.\mathrm{MHz}, \mathrm{CDCl}_{3}\right) \delta 167.9,166.3,161.0,139.0,137.2,135.9,135.3,131.0,129.1,128.8$, $128.6,128.5,128.3,128.1,126.9,125.5,119.2,118.9,115.4,76.2,75.0,53.0,52.4$, 21.6. HRMS (ESI) calculated for $\mathrm{C}_{28} \mathrm{H}_{26} \mathrm{NO}_{5}[\mathrm{M}+\mathrm{H}]^{+}: 456.1805$, found: 456.1801 .

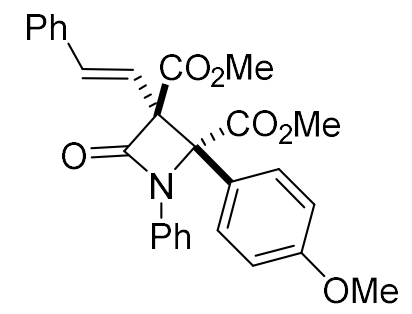

Dimethyl2-(4-methoxyphenyl)-4-oxo-1-phenyl-3-((E)-styryl)azetidine-2,3-dicarboxy late (5i)

The title compound was prepared via general procedure using $21.8 \mathrm{mg}$ of $\mathrm{N}$-phenylhydroxylamine (1a), 5i was isolated as a white solid (86.7 $\mathrm{mg}, 92 \%), \mathrm{mp}$ : 151-153 ${ }^{\circ} \mathrm{C} .{ }^{1} \mathrm{H}$ NMR $\left(400 \mathrm{MHz}, \mathrm{CDCl}_{3}\right) \delta 7.65(\mathrm{~d}, J=9.0 \mathrm{~Hz}, 2 \mathrm{H}), 7.50-7.38(\mathrm{~m}$, 
4H), 7.37-7.23 (m, 5H), 7.12 (t, $J=7.4 \mathrm{~Hz}, 1 \mathrm{H}), 6.96$ (d, $J=16.4 \mathrm{~Hz}, 1 \mathrm{H}), 6.85$ (d, $J$ $=9.0 \mathrm{~Hz}, 2 \mathrm{H}), 6.55(\mathrm{~d}, J=16.4 \mathrm{~Hz}, 1 \mathrm{H}), 3.79(\mathrm{~s}, 3 \mathrm{H}), 3.59(\mathrm{~s}, 3 \mathrm{H}), 3.25(\mathrm{~s}, 3 \mathrm{H}) .{ }^{13} \mathrm{C}$ NMR $\left(100 \mathrm{MHz}, \mathrm{CDCl}_{3}\right) \delta 168.0,166.4,161.1,160.1,137.2,135.9,135.0,129.7$, $128.8,128.7,128.6,126.9,124.5,122.5,119.1,118.5,113.5,76.3,74.9,55.3,52.9$, 52.5. HRMS (ESI) calculated for $\mathrm{C}_{28} \mathrm{H}_{26} \mathrm{NO}_{6}[\mathrm{M}+\mathrm{H}]^{+}: 472.1755$, found: 472.1758 .

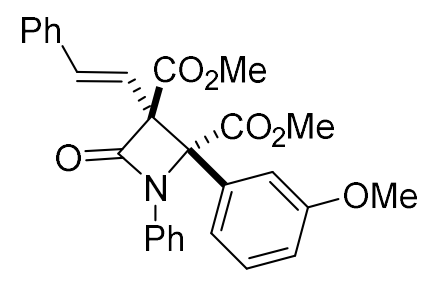

Dimethyl2-(3-methoxyphenyl)-4-oxo-1-phenyl-3-((E)-styryl)azetidine-2,3-dicarboxy late (5j)

The title compound was prepared via general procedure using $21.8 \mathrm{mg}$ of $\mathrm{N}$-phenylhydroxylamine (1a), $\mathbf{5 j}$ was isolated as a white solid $(83.0 \mathrm{mg}, 88 \%)$, mp: 118-119 ${ }^{\circ} \mathrm{C} .{ }^{1} \mathrm{H}$ NMR (400 MHz, $\left.\mathrm{CDCl}_{3}\right) \delta$ 7.52-7.39 (m, 4H), 7.38-7.22 (m, 8H), $7.12(\mathrm{t}, J=7.4 \mathrm{~Hz}, 1 \mathrm{H}), 6.96(\mathrm{~d}, J=16.4 \mathrm{~Hz}, 1 \mathrm{H}), 6.92-6.84(\mathrm{~m}, 1 \mathrm{H}), 6.56(\mathrm{~d}, J=$ $16.4 \mathrm{~Hz}, 1 \mathrm{H}), 3.74(\mathrm{~s}, 3 \mathrm{H}), 3.60(\mathrm{~s}, 3 \mathrm{H}), 3.24(\mathrm{~s}, 3 \mathrm{H}) .{ }^{13} \mathrm{C} \mathrm{NMR}\left(100 \mathrm{MHz}, \mathrm{CDCl}_{3}\right) \delta$ $167.7,166.2$, 162.6, 161.0, 159.3, 137.1, 135.8, 135.2, 132.4, 129.1, 128.8, 128.6, $126.9,124.6,120.5,119.1,118.5,114.8,114.1,76.3,75.0,55.3,53.0,52.4$. HRMS (ESI) calculated for $\mathrm{C}_{28} \mathrm{H}_{26} \mathrm{NO}_{6}[\mathrm{M}+\mathrm{H}]^{+}:$472.1755, found: 472.1750 .

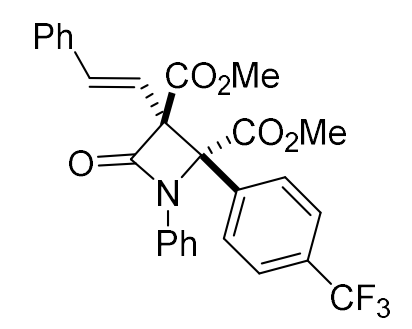

Dimethyl4-oxo-1-phenyl-3-((E)-styryl)-2-(4-(trifluoromethyl)phenyl)azetidine-2,3-di carboxylate (5k)

The title compound was prepared via general procedure using $21.8 \mathrm{mg}$ of $\mathrm{N}$-phenylhydroxylamine (1a), 5k was isolated as a light yellow solid (86.6 mg, 85\%), mp: $141-143{ }^{\circ} \mathrm{C} .{ }^{1} \mathrm{H}$ NMR $\left(400 \mathrm{MHz}, \mathrm{CDCl}_{3}\right) \delta 7.88(\mathrm{~d}, J=8.5 \mathrm{~Hz}, 2 \mathrm{H}), 7.61$ (d, $J=$ 
$8.5 \mathrm{~Hz}, 2 \mathrm{H}), 7.43$ (t, $J=7.4 \mathrm{~Hz}, 4 \mathrm{H}), 7.38-7.28(\mathrm{~m}, 5 \mathrm{H}), 7.17$ (t, $J=7.4 \mathrm{~Hz}, 1 \mathrm{H}), 6.99$ $(\mathrm{d}, J=16.4 \mathrm{~Hz}, 1 \mathrm{H}), 6.53(\mathrm{~d}, J=16.4 \mathrm{~Hz}, 1 \mathrm{H}), 3.60(\mathrm{~s}, 3 \mathrm{H}), 3.18(\mathrm{~s}, 3 \mathrm{H}) .{ }^{13} \mathrm{C} \mathrm{NMR}$ $\left(100 \mathrm{MHz}, \mathrm{CDCl}_{3}\right) \delta 167.4,166.0,160.6,136.9,135.9,135.4,135.2,131.3,131.0$, $129.01,128.95,128.8,128.8,126.9,124.91,124.88,124.8,118.2,118.1,76.5,74.3$, 53.3, 52.5. HRMS (ESI) calculated for $\mathrm{C}_{28} \mathrm{H}_{23} \mathrm{~F}_{3} \mathrm{NO}_{5}[\mathrm{M}+\mathrm{H}]^{+}$: 510.1523, found: 510.1526.

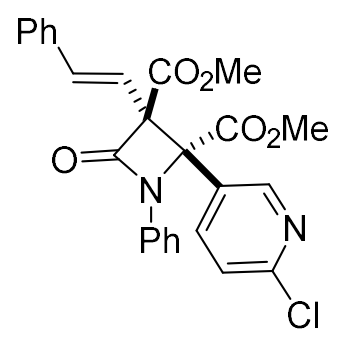

Dimethyl2-(6-chloropyridin-3-yl)-4-oxo-1-phenyl-3-((E)-styryl)azetidine-2,3-dicarb oxylate (5l)

The title compound was prepared via general procedure using $21.8 \mathrm{mg}$ of N-phenylhydroxylamine (1a), $5 \mathbf{l}$ was isolated as a white solid (72.5 mg, 76\%), mp: 145-147 ${ }^{\circ} \mathrm{C} .{ }^{1} \mathrm{H}$ NMR $\left(300 \mathrm{MHz}, \mathrm{CDCl}_{3}\right) \delta 8.69(\mathrm{~d}, J=2.7 \mathrm{~Hz}, 1 \mathrm{H}), 8.08(\mathrm{dd}, J=8.6$, $2.7 \mathrm{~Hz}, 1 \mathrm{H}), 7.47-7.28$ (m, 10H), 7.21-7.12 (m, 1H), 7.00 (d, $J=16.4 \mathrm{~Hz}, 1 \mathrm{H}), 6.50$ $(\mathrm{d}, J=16.4 \mathrm{~Hz}, 1 \mathrm{H}), 3.60$ (s, 3H), 3.31 (s, 3H). ${ }^{13} \mathrm{C} \mathrm{NMR}\left(75 \mathrm{MHz}, \mathrm{CDCl}_{3}\right) \delta 167.2$, $165.9,160.2$, 152.3, 149.6, 139.1, 136.5, 136.4, 135.5, 129.2, 129.0, 128.8, 126.9, 126.3, 125.2, 123.5, 118.0, 117.4, 76.5, 72.5, 53.5, 52.8. HRMS (ESI) calculated for $\mathrm{C}_{26} \mathrm{H}_{22} \mathrm{ClN}_{2} \mathrm{O}_{5}[\mathrm{M}+\mathrm{H}]^{+}:$477.1212, found: 477.1217.

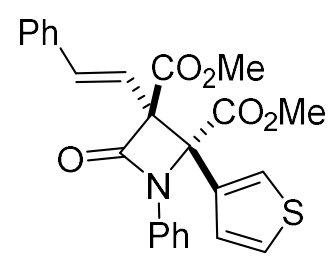

Dimethyl4-oxo-1-phenyl-3-((E)-styryl)-2-(thiophen-3-yl)azetidine-2,3-dicarboxylate $(5 m)$

The title compound was prepared via general procedure using $21.8 \mathrm{mg}$ of N-phenylhydroxylamine (1a), 5m was isolated as a white solid (63.5 $\mathrm{mg}, 71 \%), \mathrm{mp}$ : 
113-114 ${ }^{\circ} \mathrm{C} .{ }^{1} \mathrm{H}$ NMR (400 MHz, $\left.\mathrm{CDCl}_{3}\right) \delta$ 7.67-7.60 (m, 1H), 7.47-7.40 (m, 4H), 7.35-7.25 (m, 7H), 7.13 (t, $J=7.4 \mathrm{~Hz}, 1 \mathrm{H}), 6.95$ (d, $J=16.4 \mathrm{~Hz}, 1 \mathrm{H}), 6.51$ (d, $J=$ $16.4 \mathrm{~Hz}, 1 \mathrm{H}), 3.62(\mathrm{~s}, 3 \mathrm{H}), 3.34(\mathrm{~s}, 3 \mathrm{H}) .{ }^{13} \mathrm{C} \mathrm{NMR}\left(75 \mathrm{MHz}, \mathrm{CDCl}_{3}\right) \delta 167.6,166.3$, $160.8,136.7,135.8,135.5,131.8,128.8,128.73$, 128.66, 127.9, 126.9, 126.4, 125.4, 124.7, 118.5, 118.4, 76.2, 73.0, 53.0, 52.6. HRMS (ESI) calculated for $\mathrm{C}_{25} \mathrm{H}_{22} \mathrm{NO}_{5} \mathrm{~S}$ $[\mathrm{M}+\mathrm{H}]^{+}:$448.1213, found: 448.1216 .

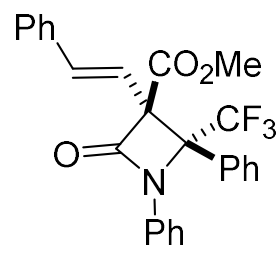

Methyl4-oxo-1,2-diphenyl-3-((E)-styryl)-2-(trifluoromethyl)azetidine-3-carboxylate (5n)

The title compound was prepared via general procedure using $21.8 \mathrm{mg}$ of $\mathrm{N}$-phenylhydroxylamine (1a), 5n was isolated as colorless oil $(74.9 \mathrm{mg}, 83 \%) .{ }^{1} \mathrm{H}$ NMR (400 MHz, $\left.\mathrm{CDCl}_{3}\right) \delta 7.58(\mathrm{~d}, J=8.0 \mathrm{~Hz}, 2 \mathrm{H}), 7.50-7.43(\mathrm{~m}, 2 \mathrm{H}), 7.42-7.37(\mathrm{~m}$, $3 \mathrm{H}), 7.35-7.28(\mathrm{~m}, 2 \mathrm{H}), 7.20-7.14(\mathrm{~m}, 4 \mathrm{H}), 6.93-6.88(\mathrm{~m}, 2 \mathrm{H}), 6.80(\mathrm{~d}, J=16.2 \mathrm{~Hz}$, $1 \mathrm{H}), 5.56(\mathrm{~d}, J=16.2 \mathrm{~Hz}, 1 \mathrm{H}), 3.88(\mathrm{~s}, 3 \mathrm{H}) .{ }^{13} \mathrm{C} \mathrm{NMR}\left(100 \mathrm{MHz}, \mathrm{CDCl}_{3}\right) \delta 166.5$, $161.4,136.2$, 135.7, 134.0, 129.7, 129.6, 129.1, 128.9, 128.5, 128.3, 127.9, 126.6, 125.5, 123.4, 120.6, 119.2, 75.3, 75.0, 72.7, 53.4. HRMS (ESI) calculated for $\mathrm{C}_{26} \mathrm{H}_{21} \mathrm{~F}_{3} \mathrm{NO}_{3}[\mathrm{M}+\mathrm{H}]^{+}$: 452.1468, found: 452.1466 .

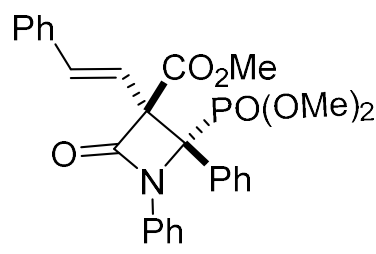

Methyl2-(dimethoxyphosphoryl)-4-oxo-1,2-diphenyl-3-((E)-styryl)azetidine-3-carbo xylate (5o)

The title compound was prepared at $80{ }^{\circ} \mathrm{C}$ for $2 \mathrm{~h}$ via general procedure using 21.8 mg of N-phenylhydroxylamine (1a), 50 was isolated as colorless oil (54.1 mg, 55\%). ${ }^{1} \mathrm{H}$ NMR (400 MHz, $\left.\mathrm{CDCl}_{3}\right) \delta 8.07(\mathrm{~s}, 1 \mathrm{H}), 7.77(\mathrm{~d}, J=8.2 \mathrm{~Hz}, 2 \mathrm{H}), 7.54(\mathrm{~d}, J=7.5$ 
$\mathrm{Hz}, 2 \mathrm{H}), 7.46-7.22(\mathrm{~m}, 9 \mathrm{H}), 7.18-7.08(\mathrm{~m}, 2 \mathrm{H}), 6.95(\mathrm{~d}, J=16.4 \mathrm{~Hz}, 1 \mathrm{H}), 3.59-3.51$ (m, 6H), $3.11(\mathrm{~s}, 3 \mathrm{H}) .{ }^{13} \mathrm{C} \mathrm{NMR}\left(100 \mathrm{MHz}, \mathrm{CDCl}_{3}\right) \delta 166.4,162.0,137.6,136.5$, $133.4,132.4,132.3,129.0,128.8,128.7,128.3,128.2,127.0,124.9,120.5,119.2$, 75.9, 72.7, 54.4, 53.4, 52.2. HRMS (ESI) calculated for $\mathrm{C}_{27} \mathrm{H}_{27} \mathrm{NO}_{6} \mathrm{P}[\mathrm{M}+\mathrm{H}]^{+}$: 492.1571, found: 492.1576 .

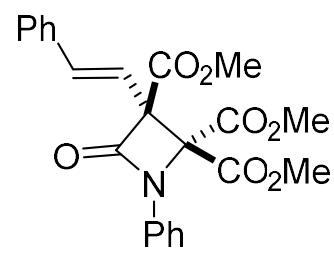

Trimethyl 4-oxo-1-phenyl-3-styrylazetidine-2,2,3-tricarboxylate (5p)

The title compound was prepared at $80{ }^{\circ} \mathrm{C}$ for $7 \mathrm{~h}$ via general procedure using 21.8 mg of N-phenylhydroxylamine (1a), 5p was isolated as colorless oil (44.0 mg, 52\%). ${ }^{1} \mathrm{H}$ NMR (400 MHz, $\left.\mathrm{CDCl}_{3}\right) \delta 7.49(\mathrm{~d}, J=7.8 \mathrm{~Hz}, 2 \mathrm{H}), 7.41-7.29(\mathrm{~m}, 7 \mathrm{H}), 7.17$ (t, $J$ $=7.4 \mathrm{~Hz}, 1 \mathrm{H}), 6.95(\mathrm{~d}, J=16.3 \mathrm{~Hz}, 1 \mathrm{H}), 6.34(\mathrm{~d}, J=16.3 \mathrm{~Hz}, 1 \mathrm{H}), 3.83(\mathrm{~s}, 3 \mathrm{H}), 3.81$ (s, 3H), $3.74(\mathrm{~s}, 3 \mathrm{H}) .{ }^{13} \mathrm{C} \mathrm{NMR}\left(125 \mathrm{MHz}, \mathrm{CDCl}_{3}\right) \delta 166.7,165.7,164.9,160.3$, 136.51, 136.48, 135.3, 128.93, 128.91, 128.8, 126.9, 125.3, 118.3, 117.9, 73.3, 73.1, 53.7, 53.50, 53.46. HRMS (ESI) calculated for $\mathrm{C}_{23} \mathrm{H}_{22} \mathrm{NO}_{7}[\mathrm{M}+\mathrm{H}]^{+}:$424.1391, found: 424.1394.

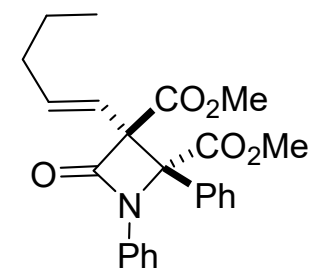

Dimethyl4-oxo-3-((E)-pent-1-en-1-yl)-1,2-diphenylazetidine-2,3-dicarboxylate (5q)

The title compound was prepared via general procedure using $21.8 \mathrm{mg}$ of N-phenylhydroxylamine (1a), 5q was isolated as a white solid (59.5 mg, 73\%), mp: 178-179 ${ }^{\circ} \mathrm{C} .{ }^{1} \mathrm{H}$ NMR $\left(400 \mathrm{MHz}, \mathrm{CDCl}_{3}\right) \delta 7.70-7.62(\mathrm{~m}, 2 \mathrm{H}), 7.42(\mathrm{~d}, J=8.0 \mathrm{~Hz}$, 2H), 7.36-7.24 (m, 5H), 7.11 (t, $J=7.3 \mathrm{~Hz}, 1 \mathrm{H}), 6.18-6.08(\mathrm{~m}, 1 \mathrm{H}), 5.86(\mathrm{~d}, J=15.9$ $\mathrm{Hz}, 1 \mathrm{H}), 3.73$ (s, 3H), 3.14 (s, 3H), 2.14-2.04 (m, 2H), 1.48-1.37 (m, 2H), 0.91 (t, $J=$ $7.4 \mathrm{~Hz}, 3 \mathrm{H}) .{ }^{13} \mathrm{C} \mathrm{NMR}\left(100 \mathrm{MHz}, \mathrm{CDCl}_{3}\right) \delta 167.8,166.5,161.3,137.9,137.3,131.2$, 
$128.9,128.7,128.2,128.0,124.4,119.7,118.4,76.1,74.7,52.7,52.2,35.0,22.0,13.8$. HRMS (ESI) calculated for $\mathrm{C}_{24} \mathrm{H}_{26} \mathrm{NO}_{5}[\mathrm{M}+\mathrm{H}]^{+}: 408.1805$, found: 408.1803 .

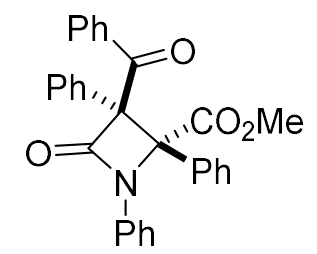

\section{Methyl 3-benzoyl-4-oxo-1,2,3-triphenylazetidine-2-carboxylate (5r)}

The title compound was prepared at $80{ }^{\circ} \mathrm{C}$ for $6 \mathrm{~h}$ via general procedure using 21.8 mg of N-phenylhydroxylamine (1a), 5r was isolated as a white solid (34.1 mg, 37\%), mp: 197-199 ${ }^{\circ} \mathrm{C} .{ }^{1} \mathrm{H}$ NMR $\left(400 \mathrm{MHz}, \mathrm{CDCl}_{3}\right) \delta 7.50(\mathrm{~d}, J=7.5 \mathrm{~Hz}, 2 \mathrm{H}), 7.43-7.29(\mathrm{~m}$, $6 \mathrm{H}), 7.28-7.18(\mathrm{~m}, 9 \mathrm{H}), 7.17-7.05(\mathrm{~m}, 3 \mathrm{H}), 3.95(\mathrm{~s}, 3 \mathrm{H}) .{ }^{13} \mathrm{C} \mathrm{NMR}\left(125 \mathrm{MHz}, \mathrm{CDCl}_{3}\right)$ $\delta 171.1,163.4,160.4,137.3,133.3,133.0,132.2,131.5,131.0,130.7,130.6,129.7$, 128.4, 128.2, 128.0, 127.52, 127.48, 127.2, 113.7, 95.2, 53.8. HRMS (ESI) calculated for $\mathrm{C}_{30} \mathrm{H}_{24} \mathrm{NO}_{4}[\mathrm{M}+\mathrm{H}]^{+}: 462.1700$, found: 462.1705 .

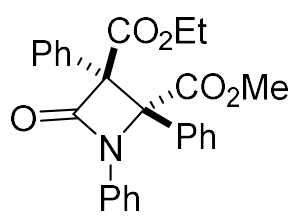

\section{3-ethyl 2-methyl (-4-oxo-1,2,3-triphenylazetidine-2,3-dicarboxylate (5s)}

The title compound was prepared via general procedure using $21.8 \mathrm{mg}$ of N-phenylhydroxylamine (1a), 5s was isolated as a white solid (48.1 mg, 56\%), mp: 202-204 ${ }^{\circ} \mathrm{C} .{ }^{1} \mathrm{H}$ NMR (400 MHz, $\left.\mathrm{CDCl}_{3}\right) \delta 7.82(\mathrm{~d}, J=7.4 \mathrm{~Hz}, 2 \mathrm{H}), 7.79-7.73(\mathrm{~m}$, 2H), 7.44-7.34 (m, 8H), $7.30(\mathrm{t}, J=7.7 \mathrm{~Hz}, 2 \mathrm{H}), 7.13(\mathrm{t}, J=7.4 \mathrm{~Hz}, 1 \mathrm{H}), 3.70-3.60$ $(\mathrm{m}, 1 \mathrm{H}), 3.59-3.49(\mathrm{~m}, 1 \mathrm{H}), 3.05(\mathrm{~s}, 3 \mathrm{H}), 0.87(\mathrm{t}, J=7.2 \mathrm{~Hz}, 3 \mathrm{H}) .{ }^{13} \mathrm{C}$ NMR $(100$ $\left.\mathrm{MHz}, \mathrm{CDCl}_{3}\right) \delta 168.2,165.9,161.7,137.3,131.6,131.3,129.1,129.0,128.9,128.6$, 128.4, 128.1, 127.9, 124.6, 117.7, 77.5, 73.7, 62.3, 52.6, 13.5. HRMS (ESI) calculated for $\mathrm{C}_{26} \mathrm{H}_{24} \mathrm{NO}_{5}[\mathrm{M}+\mathrm{H}]^{+}:$430.1649, found: 430.1646 . 


\section{Preparation of 3a in $2 \mathrm{mmol}$ scale $5 \mathrm{a}$ in $1 \mathrm{mmol}$ scale}

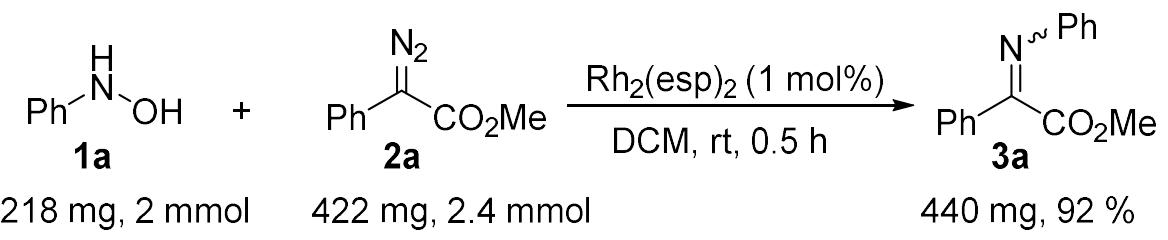

To a tube was added $\mathrm{Rh}_{2}(\mathrm{esp})_{2}(15.1 \mathrm{mg}, 0.02 \mathrm{mmol}, 0.01 \mathrm{eq}), 1 \mathrm{a}(218 \mathrm{mg}, 2 \mathrm{mmol}, 1$ eq), 2a (422 mg, $2.4 \mathrm{mmol}, 1.2 \mathrm{eq})$ and DCM (40 mL) under argon atmosphere, the resulting solution was stirred at $\mathrm{rt}$ for $0.5 \mathrm{~h}$. The reaction mixture was concentrated under vacuum, the residue was purified by column chromatography (silica gel, eluted with EtOAc: Petroleum ether $=1: 20)$ to give product 3a $(440 \mathrm{mg}, 92 \%)$ as yellow oil .

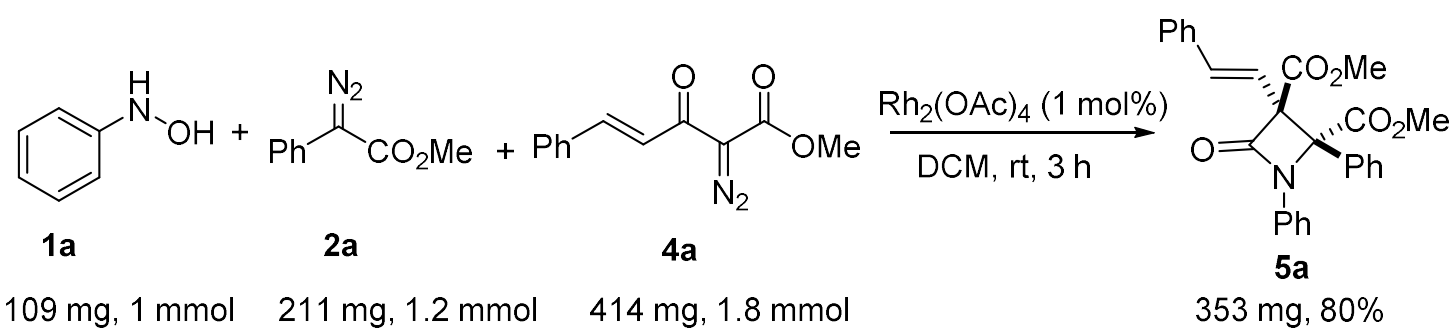

To a tube was added $\mathrm{Rh}_{2}(\mathrm{OAc})_{4}(4.4 \mathrm{mg}, 0.01 \mathrm{mmol}, 0.01 \mathrm{eq}), 1$ (109 mg, $1 \mathrm{mmol}, 1$ eq), 2 (211 mg, $1.2 \mathrm{mmol}, 1.2 \mathrm{eq}), 4$ (414 mg, $1.8 \mathrm{mmol}, 1.8 \mathrm{eq})$ and DCM (20 mL) under argon atmosphere, the resulting solution stirred at $\mathrm{rt}$ for $3 \mathrm{~h}$. The reaction mixture was concentrated under vacuum, the residue was purified by column chromatography (silica gel, eluted with EtOAc: Petroleum ether $=1: 10$ ) to give product $5 \mathbf{a}(353 \mathrm{mg}, 80 \%)$ as a white solid.

\section{$X$-ray structure of $5 q$ and $5 s$}

The crystal structures have been deposited at the Cambridge Crystallographic Data Centre (CCDC 1860110, 5q) and (CCDC 1860109, 5s). The data can be obtained free of charge via the internet at https://www.ccdc.cam.ac.uk/structures/ 


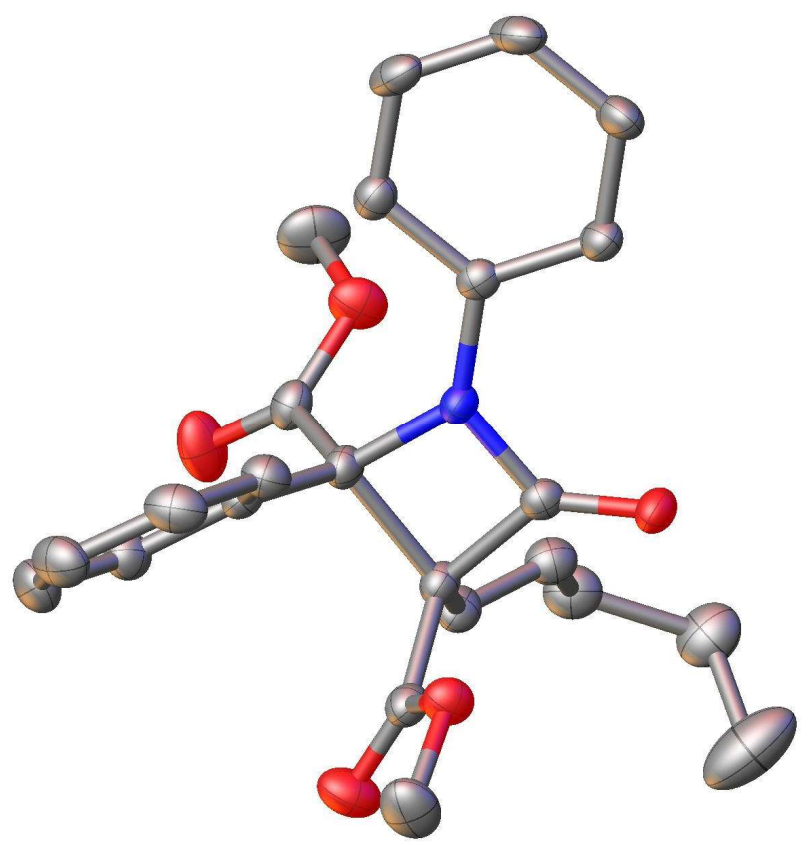

X-ray of $\mathbf{5 q}$

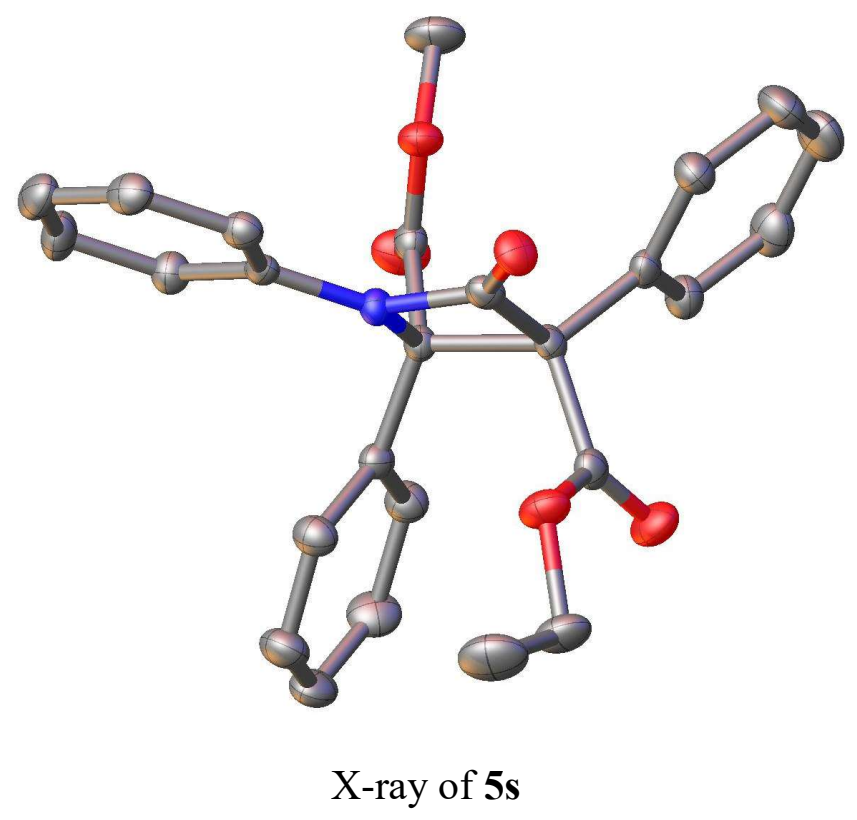

\section{References:}

1. (a) Beissel, T.; Powers, R. E.; Parac, T. N.; Raymond, K. N. J. Am. Chem. Soc. 1999, 121, 4200. (b) Sato, M.; Nagano, S.; Seki, T. Chem. Commun. 2009, 3792. (c) Quiclet-Sire, B.; Tran, N. D. M.; Zard, S. Z. Org. Lett. 2012, 14, 5514. (d) Nakamura, I.; Jo, T.; Ishida, Y.; Tashiro, H.; Terada, M. Org. Lett. 2017, 19, 3059. (e) Lee, K. N.; Lei, Z.; Morales-Rivera, C. A.; Liu, P.; Ngai, M.-Y. Org. 
Biomol. Chem. 2016, 14, 5599. (f) Kjaer, A. M.; Ulstrup, J. J. Am. Chem. Soc. 1988, 110, 3874.

2. (a) Schwartz, B. D.; Denton, J. R.; Lian, Y.; Davies, H. M. L.; Williams, C. M. J. Am. Chem. Soc. 2009, 131, 8329. (b) Davies, H. M. L.; Hansen, T.; Churchill, M. R. J. Am. Chem. Soc. 2000, 122, 3063. (c) Seyferth, D.; Marmor, Robert S.; Hilbert, P. J. Org. Chem. 1971, 36, 1379. (d) Emer, E.; Twilton, J.; Tredwell, M.; Calderwood, S.; Collier, T. L.; Liegault, B.; Taillefer, M.; Gouverneur, V. Org. Lett. 2014, 16, 6004. (e) Gratia, S.; Mosesohn, K.; Diver, S. T. Org. Lett. 2016, 18 , 5320.

3. (a) Chen, X.; Xie, Y.; Xiao, X.; Li, G.; Deng, Y.; Jiang, H.; Zeng, W. Chem. Commun. 2015, 51, 15328. (b) Shanahan, C. S.; Truong, P.; Mason, S. M.; Leszczynski, J. S.; Doyle, M. P. Org. Lett. 2013, 15, 3642.

4. Mandler, M. D.; Truong, P. M.; Zavalij, P. Y.; Doyle, M. P. Org. Lett. 2014, 16, 740 .

5. Tindall, D. J.; Werlé, C.; Goddard, R.; Philipps, P.; Farès, C.; Fürstner, A. J. Am. Chem. Soc. 2018, 140, 1884.

6. Huang, H.; Wang, Y.; Chen, Z.; Hu, W. Adv. Synth. Catal. 2005, 347, 531.

7. Li, C.-L.; Chen, M.-W.; Zhang, X.-G. J. Fluorine Chem. 2010, 131, 856.

8. Sidky, M. M.; Soliman, F. M.; Shabana, R. Tetrahedron, 1971, 27, 3431.

9. Reddy, C. R. V.; Urgaonkar, S.; Verkade, J. G. Org. Lett. 2005, 7, 4427.

10. Wang, H.; Wang, C.; Huang, K.; Liu, L.; Chang, W.; Li, J. Org. Lett. 2016, 18, 2367.

11. Song, E.; Kim, H. Y.; Oh, K. Org. Lett. 2017, 19, 5264.

12. Sirois, J. J.; Davis, R.; DeBoef, B. Org. Lett. 2014, 16, 868.

\section{Density functional theory (DFT) calculations}

\section{Supplementary Note 1. Computational methods}

All DFT computations were carried out with the Gaussian 09 program package. ${ }^{1}$ Geometry optimizations were performed using B3LYP density functional ${ }^{2-4}$ with Grimme's dispersion correction D3 ${ }^{5}$. The Lanl2DZ $+\mathrm{f}(1.35)$ basis set with effective core potential (ECP) was adopted for the Rh atom. ${ }^{6-7}$ For all the other atoms, the 6-31G(d) basis set ${ }^{8-9}$ was used. Solvent effects were taken into account during geometry optimizations via $\mathrm{SMD}^{10}$ in dichloromethane. Frequency analyses were performed at the same level to obtain thermal corrections at $298.15 \mathrm{~K}$, and to confirm the stationary points with zero imaginary frequency and transition states with only one imaginary frequency. All structures shown here in figures were generated with the CYLview program. ${ }^{11}$ 


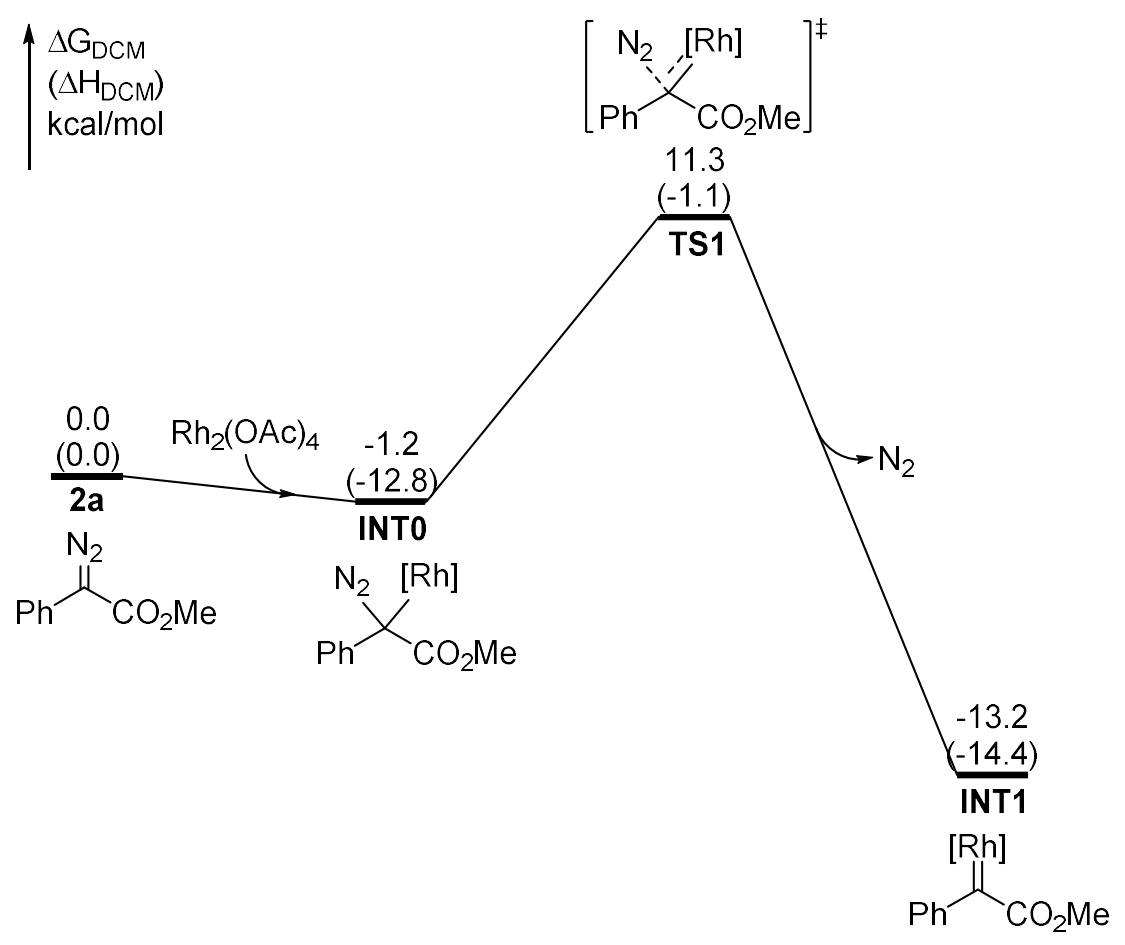

Supplementary Figure 1. The free energy profile for the $\mathrm{Rh}_{2}(\mathrm{OAc})_{4}$ catalyzed rhodium carbene formation. Free energies and enthalpies (in parenthesis) are given in $\mathrm{kcal} / \mathrm{mol}$.

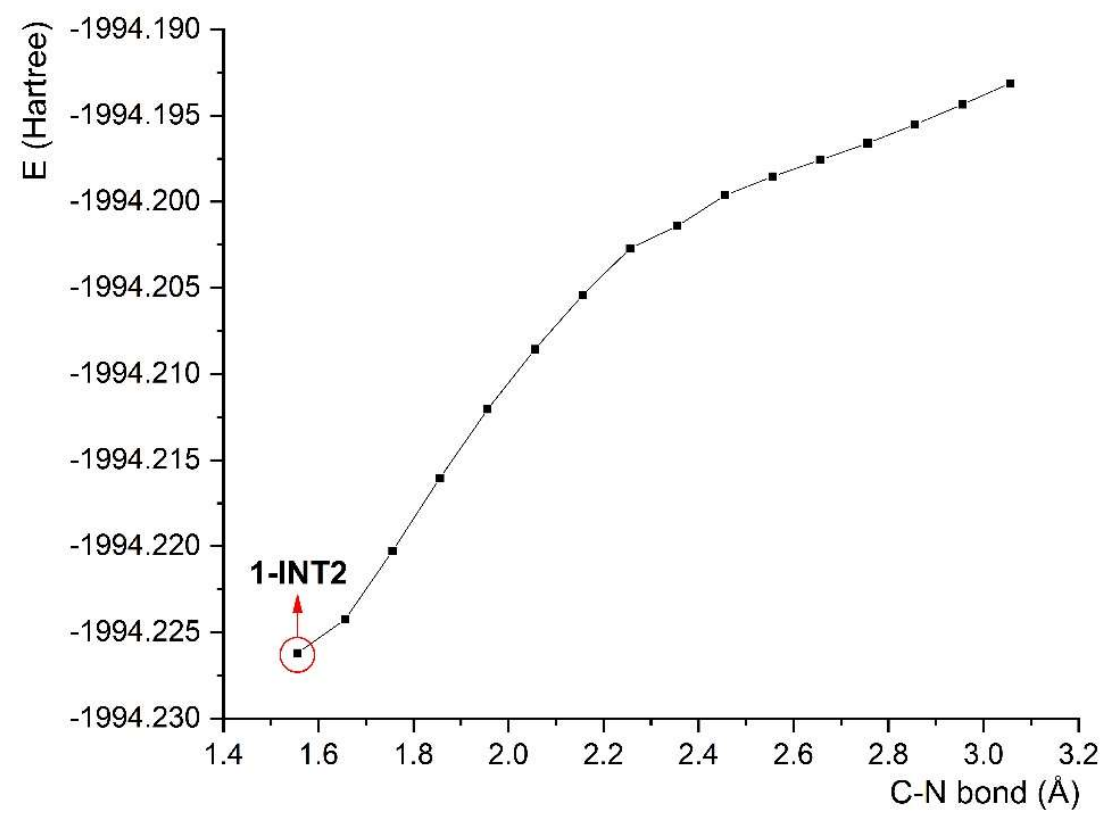

Supplementary Figure 2. Scanning profile for the C-N bond formation between 1a and INT1 to give 1-INT2. Electronic energies (E) are given in Hartree and bond lengths are in $\AA$.E 
$[1,2]-\mathrm{H}$ shift<smiles>COC(=O)C(c1ccccc1)[N+](O)c1ccccc1</smiles>

2-TS3_O $\mathrm{H}_{2} \mathrm{O}$

35.7

(34.0)

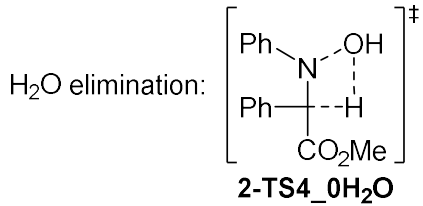

19.9

(18.4)

[1,4]-H shift: $\quad\left[\begin{array}{ccc}\text { (Rh] } & \text { OMe }\end{array}\right]$

3-TS2_O $\mathrm{H}_{2} \mathrm{O}$

$-9.9$

$(-27.0)$

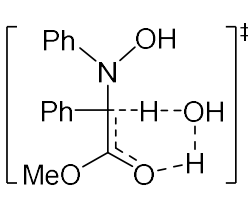

3-TS3_1 $\mathrm{H}_{2} \mathrm{O}$

12.5

$(-0.6)$

$[1,3]-\mathrm{H}$ shift

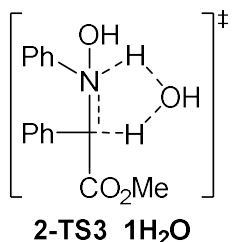

12.5

$(-0.7)$

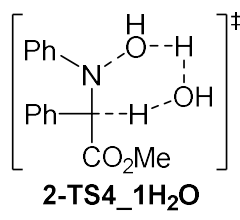

9.7

$(-2.4)$

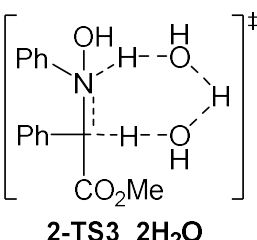

1.1

$(-22.9)$

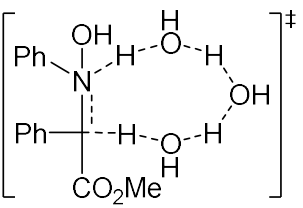

2-TS3

$-1.2$

(-35.6)
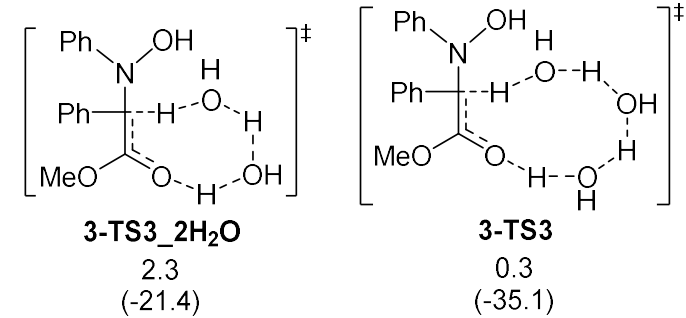

Supplementary Figure 3. The transition states for the directly or $\mathrm{H}_{2} \mathrm{O}$ catalyzed [1,2]- $\mathrm{H}$ shift, [1,3]- $\mathrm{H}$ shift, [1,4]-H shift and $\mathrm{H}_{2} \mathrm{O}$ elimination. Free energies and enthalpies (in parenthesis) are given in $\mathrm{kcal} / \mathrm{mol}$. 


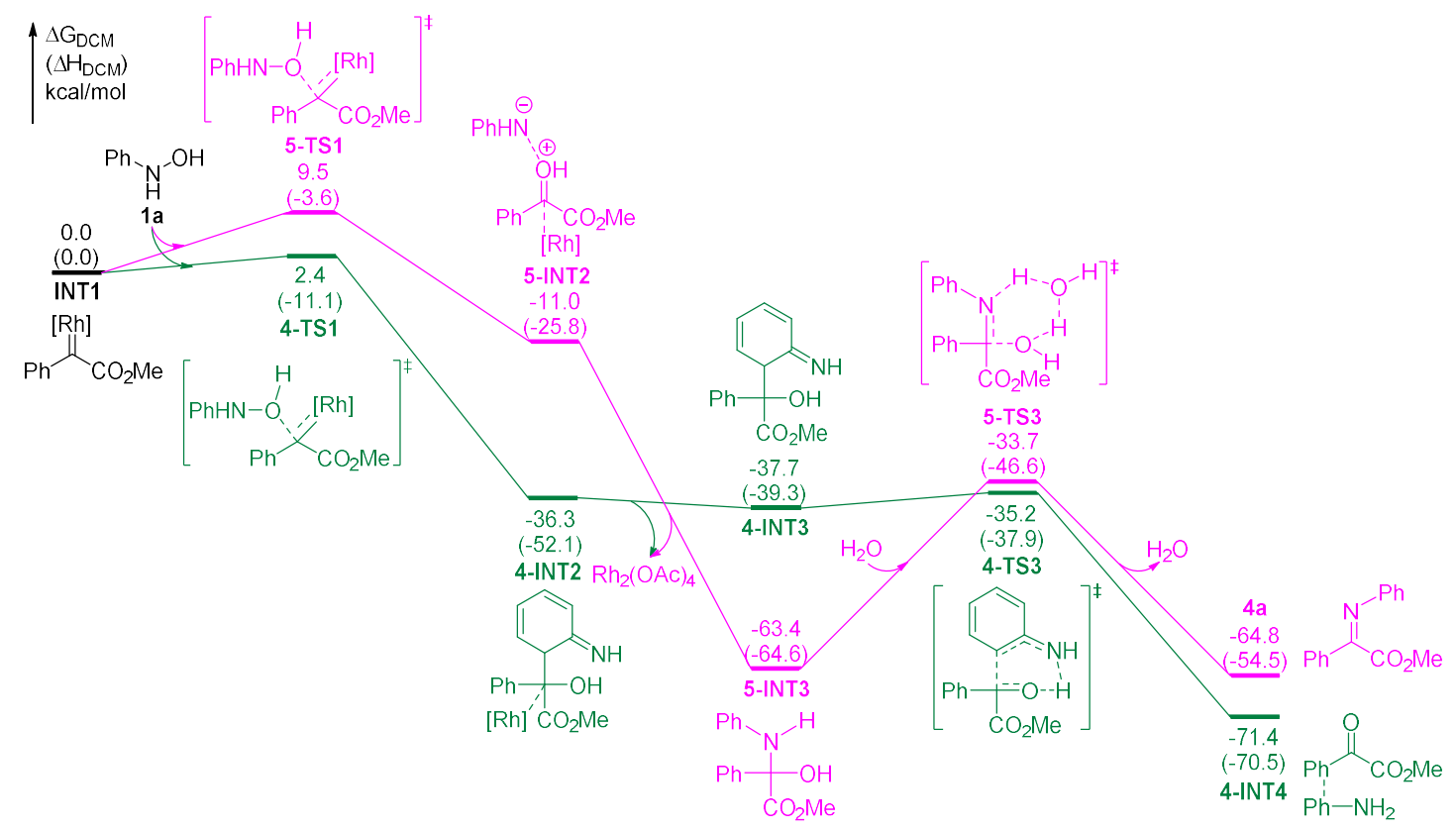

Supplementary Figure 4. The free energy profile of the reaction between 1a and INT1, starting with the nucleophilic attack of $\mathrm{O}$ atom in 1a to INT1 (mechanism 4 and 5), which shows different regioselectivity of 1a than the mechanisms (mechanism 1,2 and 3) in the main text. Free energies and enthalpies (in parenthesis) are given in $\mathrm{kcal} / \mathrm{mol}$.

\section{Supplementary References}

1. Gaussian 09, Revision D.01, M. J. Frisch, G. W. Trucks, H. B. Schlegel, G. E. Scuseria, M. A. Robb, J. R. Cheeseman, G. Scalmani, V. Barone, G. A. Petersson, H. Nakatsuji, X. Li, M. Caricato, A. Marenich, J. Bloino, B. G. Janesko, R. Gomperts, B. Mennucci, H. P. Hratchian, J. V. Ortiz, A. F. Izmaylov, J. L. Sonnenberg, D. Williams-Young, F. Ding, F. Lipparini, F. Egidi, J. Goings, B. Peng, A. Petrone, T. Henderson, D. Ranasinghe, V. G. Zakrzewski, J. Gao, N. Rega, G. Zheng, W. Liang, M. Hada, M. Ehara, K. Toyota, R. Fukuda, J. Hasegawa, M. Ishida, T. Nakajima, Y. Honda, O. Kitao, H. Nakai, T. Vreven, K. Throssell, J. A. Montgomery, Jr., J. E. Peralta, F. Ogliaro, M. Bearpark, J. J. Heyd, E. Brothers, K. N. Kudin, V. N. Staroverov, T. Keith, R. Kobayashi, J. Normand, K. Raghavachari, A. Rendell, J. C. Burant, S. S. Iyengar, J. Tomasi, M. Cossi, J. M. Millam, M. Klene, C. Adamo, R. Cammi, J. W. Ochterski, R. L. Martin, K. Morokuma, O. Farkas, J. B. Foresman, and D. J. Fox, Gaussian, Inc., Wallingford CT, 2016.

2. Becke, A. D., J. Chem. Phys. 1993, 98, 5648-5652.

3. Lee, C.; Yang, W.; Parr, R. G., Phys. Rev. B 1988, 37, 785-789.

4. Stephens, P. J.; Devlin, F. J.; Chabalowski, C. F.; Frisch, M. J., J. Phys. Chem. 1994, 98, 11623-11627.

5. Grimme, S.; Antony, J.; Ehrlich, S.; Krieg, H., J. Chem. Phys. 2010, 132, 154104.

6. Hay, P. J.; Wadt, W. R., J. Chem. Phys. 1985, 82, 270-283. 
7. Ehlers, A. W.; Böhme, M.; Dapprich, S.; Gobbi, A.; Höllwarth, A.; Jonas, V.; Köhler, K. F.; Stegmann, R.; Veldkamp, A.; Frenking, G., Chem. Phys. Lett. 1993, 208, 111-114.

8. Hariharan, P. C.; Pople, J. A., Theor. Chim. Acta 1973, 28, 213-222.

9. Francl, M. M.; Pietro, W. J.; Hehre, W. J.; Binkley, J. S.; Gordon, M. S.; DeFrees, D. J.; Pople, J. A., J. Chem. Phys. 1982, 77, 3654-3665.

10. Marenich, A. V.; Cramer, C. J.; Truhlar, D. G., J. Phys. Chem. B 2009, 113, 6378-6396.

11. C. Y. Legault, CYLview, 1.0b, Universite de Sherbrooke, 2009, http://www.cylview.org.

12. Krishnan, R.; Binkley, J. S.; Seeger, R.; Pople, J. A., J. Chem. Phys. 1980, 72, 650-654.

13. McLean, A. D.; Chandler, G. S., J. Chem. Phys. 1980, 72, 5639-5648.

14. Clark, T.; Chandrasekhar, J.; Spitznagel, G. W.; Schleyer, P. V. R., J. Comput. Chem. 1983, 4, 294-301.

15. Weigend, F.; Ahlrichs, R., Phys. Chem. Chem. Phys. 2005, 7, 3297-3305.

Supplementary Table 1. Energies of structures on reaction between 2a and $\mathrm{Rh}_{2}(\mathrm{OAc})_{4}$ (units of $\mathrm{H}$ and $\mathrm{G}$ are Hartree, $\Delta \mathrm{H}$ and $\Delta \mathrm{G}$ are $\mathrm{kcal} / \mathrm{mol}$ ).

\begin{tabular}{l|cccc}
\hline & $\mathrm{H}$ & $\mathrm{G}$ & $\Delta \mathrm{H}$ & $\Delta \mathrm{G}$ \\
\hline $\mathrm{Rh}_{2}(\mathrm{OAc})_{4}$ & -1132.949145 & -1133.021085 & 0.0 & 0.0 \\
$\mathrm{~N}_{2}$ & -109.506703 & -109.528457 & $/$ & $/$ \\
$\mathbf{2 a}$ & -607.525386 & -607.576599 & 0.0 & 0.0 \\
INT0 & -1740.494881 & -1740.599531 & -12.8 & -1.2 \\
TS1 & -1740.476316 & -1740.57973 & -1.1 & 11.3 \\
INT1 & -1630.99077 & -1631.09033 & -14.4 & -13.2 \\
\hline
\end{tabular}

Supplementary Table 2. Energies of structures on reaction between 1a and INT1 (units of $H$ and $\mathrm{G}$ are Hartree, $\Delta \mathrm{H}$ and $\Delta \mathrm{G}$ are $\mathrm{kcal} / \mathrm{mol}$ ).

\begin{tabular}{l|cccc}
\hline & $\mathrm{H}$ & $\mathrm{G}$ & $\Delta \mathrm{H}$ & $\Delta \mathrm{G}$ \\
\hline $\mathrm{Rh}_{2}(\mathrm{OAc})_{4}$ & -1132.949145 & -1133.021085 & $/$ & $/$ \\
$\mathrm{H}_{2} \mathrm{O}$ & -76.389557 & -76.411012 & $/$ & $/$ \\
$\mathbf{1 a}$ & -362.646574 & -362.685285 & 0.0 & 0.0 \\
$\mathbf{I N T 1}$ & -1630.99077 & -1631.09033 & 0.0 & 0.0 \\
$\mathbf{1 - I N T 2}$ & -1993.697585 & -1993.80903 & -37.8 & -21.0 \\
$\mathbf{1 - T S 2}$ & -1993.679323 & -1993.793477 & -26.3 & -11.2
\end{tabular}




\begin{tabular}{|c|c|c|c|c|}
\hline 1-INT3 & -1993.678458 & -1993.79348 & -25.8 & -11.2 \\
\hline 1-TS3 & -1993.679667 & -1993.792934 & -26.6 & -10.9 \\
\hline 2-TS3_OH ${ }_{2} \mathrm{O}$ & -860.634087 & -860.697595 & 34.0 & 35.7 \\
\hline 2-TS3_1 $1 \mathrm{H}_{2} \mathrm{O}$ & -937.078853 & -937.145665 & -0.7 & 12.5 \\
\hline $2-\mathrm{TS} 3 \_2 \mathrm{H}_{2} \mathrm{O}$ & -1013.503813 & -1013.574729 & -22.9 & 1.1 \\
\hline 2-TS3 & -1089.913557 & -1089.989467 & -35.6 & -1.2 \\
\hline 2-INT4 & -860.740318 & -860.80556 & -32.7 & -32.0 \\
\hline 2-TS4_OH & -860.658891 & -860.722843 & 18.4 & 19.9 \\
\hline 2-TS4_1 $1 \mathrm{H}_{2} \mathrm{O}$ & -937.081571 & -937.150152 & -2.4 & 9.7 \\
\hline 2-TS4 & -1013.491745 & -1013.563141 & -15.3 & 8.4 \\
\hline 3-TS2_OH & -1993.680448 & -1993.791466 & -27.0 & -9.9 \\
\hline 3-TS2 & -2070.095977 & -2070.210777 & -43.3 & -15.2 \\
\hline 3-INT3 & -860.705381 & -860.768791 & -10.8 & -8.9 \\
\hline 3-TS3_1 $1 \mathrm{H}_{2} \mathrm{O}$ & -937.078674 & -937.145637 & -0.6 & 12.5 \\
\hline 3-TS3_2 $\mathrm{H}_{2} \mathrm{O}$ & -1013.501396 & -1013.572856 & -21.4 & 2.3 \\
\hline 3-TS3 & -1089.912784 & -1089.987035 & -35.1 & 0.3 \\
\hline 4-TS1 & -1993.655106 & -1993.7718 & -11.1 & 2.4 \\
\hline 4-INT2 & -1993.720364 & -1993.833385 & -52.1 & -36.3 \\
\hline 4-INT3 & -860.750875 & -860.814606 & -39.3 & -37.7 \\
\hline 4-TS3 & -860.748548 & -860.810632 & -37.9 & -35.2 \\
\hline 4-INT4 & -860.800503 & -860.868287 & -70.5 & -71.4 \\
\hline 5-TS1 & -1993.643099 & -1993.760462 & -3.6 & 9.5 \\
\hline 5-INT2 & -1993.678408 & -1993.793128 & -25.8 & -11.0 \\
\hline 5-INT3 & -860.7912 & -860.85561 & -64.6 & -63.4 \\
\hline 5-TS3 & -937.151948 & -937.219259 & -46.6 & -33.7 \\
\hline $4 a$ & -784.3855 & -784.446707 & -54.5 & -64.8 \\
\hline
\end{tabular}

Supplementary Note 2. Cartesian coordinate

$\mathrm{Rh}_{2}(\mathrm{OAc})_{4}$

$\begin{array}{lrrr}\mathrm{Rh} & 0.00026500 & -0.00084100 & -1.19500900 \\ \mathrm{Rh} & -0.00249100 & -0.00135600 & 1.19654700\end{array}$




\begin{tabular}{|c|c|c|c|}
\hline $\mathrm{O}$ & 1.41388800 & 1.49680400 & -1.13017000 \\
\hline $\mathrm{C}$ & 1.82350400 & 1.91070000 & 0.00383700 \\
\hline $\mathrm{O}$ & 1.41227400 & 1.49463200 & 1.13622000 \\
\hline $\mathrm{O}$ & 1.49170200 & -1.41778600 & 1.13797800 \\
\hline $\mathrm{C}$ & 1.90968000 & -1.82644600 & 0.00575700 \\
\hline $\mathrm{O}$ & 1.49654600 & -1.41572000 & -1.12843500 \\
\hline $\mathrm{O}$ & -1.49819900 & 1.41352900 & 1.13236600 \\
\hline $\mathrm{C}$ & -1.91287100 & 1.82328100 & -0.00100200 \\
\hline $\mathrm{O}$ & -1.49746600 & 1.41236600 & -1.13405100 \\
\hline $\mathrm{O}$ & -1.41706600 & -1.49773400 & 1.13101100 \\
\hline $\mathrm{C}$ & -1.82559600 & -1.91253900 & -0.00312400 \\
\hline $\mathrm{O}$ & -1.41324800 & -1.49751400 & -1.13541700 \\
\hline $\mathrm{C}$ & 2.97957500 & -2.88772600 & -0.00565200 \\
\hline $\mathrm{H}$ & 2.59713000 & -3.77765800 & -0.51750900 \\
\hline $\mathrm{H}$ & 3.84522000 & -2.52656500 & -0.57083100 \\
\hline $\mathrm{H}$ & 3.28150000 & -3.14890700 & 1.01003100 \\
\hline $\mathrm{C}$ & -2.91083500 & -2.95795000 & -0.00069800 \\
\hline $\mathrm{H}$ & -3.87818800 & -2.45523900 & 0.12227800 \\
\hline $\mathrm{H}$ & -2.92030000 & -3.50753200 & -0.94434600 \\
\hline $\mathrm{H}$ & -2.77581600 & -3.64354500 & 0.83971800 \\
\hline $\mathrm{C}$ & -2.95847900 & 2.90834600 & -0.00579200 \\
\hline $\mathrm{H}$ & -2.45586700 & 3.87566100 & -0.12947400 \\
\hline $\mathrm{H}$ & -3.64346400 & 2.77218700 & -0.84650400 \\
\hline $\mathrm{H}$ & -3.50871000 & 2.91887800 & 0.93748000 \\
\hline $\mathrm{C}$ & 2.90881600 & 2.95607700 & 0.00149000 \\
\hline $\mathrm{H}$ & 2.77478000 & 3.64091400 & -0.83968600 \\
\hline $\mathrm{H}$ & 2.91743600 & 3.50645100 & 0.94469300 \\
\hline $\mathrm{H}$ & 3.87625700 & 2.45317200 & -0.11996600 \\
\hline \multicolumn{4}{|l|}{$\mathrm{N}_{2}$} \\
\hline $\mathrm{N}$ & 0.00000000 & 0.00000000 & 0.55242300 \\
\hline $\mathrm{N}$ & 0.00000000 & 0.00000000 & -0.55242300 \\
\hline \multicolumn{4}{|c|}{$\mathrm{H}_{2} \mathrm{O}$} \\
\hline $\mathrm{O}$ & 0.00000000 & 0.00000000 & 0.12052200 \\
\hline $\mathrm{H}$ & 0.00000000 & 0.76171100 & -0.48209000 \\
\hline $\mathrm{H}$ & 0.00000000 & -0.76171100 & -0.48209000 \\
\hline \multicolumn{4}{|l|}{$1 \mathbf{a}$} \\
\hline $\mathrm{N}$ & -1.82777400 & -0.59885700 & -0.22925000 \\
\hline $\mathrm{H}$ & -2.06237500 & -1.45403900 & 0.27233600 \\
\hline $\mathrm{O}$ & -2.70534100 & 0.40822600 & 0.27839700 \\
\hline $\mathrm{H}$ & -3.19574900 & 0.69119400 & -0.51402300 \\
\hline $\mathrm{C}$ & -0.46332000 & -0.26384800 & -0.07718900 \\
\hline
\end{tabular}




$\begin{array}{lrrr}\mathrm{C} & -0.02664700 & 1.06857400 & -0.08066100 \\ \mathrm{C} & 0.48174600 & -1.30192900 & -0.00381000 \\ \mathrm{C} & 1.33923000 & 1.35174900 & -0.01568100 \\ \mathrm{H} & -0.75859800 & 1.86721600 & -0.11998700 \\ \mathrm{C} & 1.84259400 & -1.00496700 & 0.05223700 \\ \mathrm{H} & 0.14446200 & -2.33625100 & 0.00399500 \\ \mathrm{C} & 2.28282000 & 0.32308100 & 0.04864400 \\ \mathrm{H} & 1.66467700 & 2.38924100 & -0.01254800 \\ \mathrm{H} & 2.56225900 & -1.81798400 & 0.10819700 \\ \mathrm{H} & 3.34393400 & 0.55085200 & 0.09837000\end{array}$

2a

C

C

$\mathrm{N}$

$\mathrm{N}$

C

$$
\begin{array}{r}
1.62289500 \\
0.50020400 \\
0.85116600 \\
1.13378600 \\
-0.94296900 \\
-1.39661000 \\
-2.76376600 \\
-3.70481600 \\
-3.26126300 \\
-1.89888100 \\
-1.58433800 \\
-3.97735800 \\
-4.76752100 \\
-3.09049100 \\
-0.68024400 \\
1.51293400 \\
2.81089600 \\
3.97753600 \\
3.99516400 \\
4.82834700 \\
3.99717200
\end{array}
$$

$-0.55210800$

0.39658100

0.02734200

1.66485600

$-0.02619400$

2.76521400

$-0.07714000$

0.07766900

$-0.12209100$

$-1.25022500$

$-0.00810800$

$-1.53021400$

$-0.12128000$

$-0.50610600$

$-0.10708000$

0.81462200

0.01535600

1.10590500

0.12588900

2.14192200

0.11648400

1.62636200

0.20884400

$-0.73189000$

0.22317400

$-2.56321400$

0.02422100

$-2.05507500$

$-0.19538200$

$-1.76532900$

$-0.21551600$

0.09070100

0.08390400

$-0.75441900$

0.00816700

$-1.43029000$

0.04986000

$-0.07313200$

$-0.80974800$

$-1.33838700$

0.01434300

0.97450700

\section{INT0}

$\mathrm{Rh}$

C

C

$\mathrm{O}$

C

C

C

C

C

0.10930300

0.09523400

1.29415600

0.57219300

2.51908700

$-0.22872600$

2.70171000

$-1.36804900$

0.29023500

0.25022600

0.12741900

$-1.07110700$

$-0.83583300$

$-1.36815400$

$-1.65820900$

$-0.36771000$

4.60777500

0.94536000 


\begin{tabular}{|c|c|c|c|}
\hline $\mathrm{C}$ & 3.64880000 & -0.54289300 & 1.25389900 \\
\hline $\mathrm{H}$ & 3.31116300 & -0.45203800 & 2.28159200 \\
\hline $\mathrm{H}$ & 5.00181600 & -2.13641800 & 1.73805600 \\
\hline $\mathrm{H}$ & 5.80991300 & -2.40744200 & -0.60695900 \\
\hline $\mathrm{H}$ & 4.87584400 & -0.94358300 & -2.39548700 \\
\hline $\mathrm{H}$ & 3.16676200 & 0.74765400 & -1.85672700 \\
\hline $\mathrm{O}$ & -1.07605600 & 1.09076800 & 1.63340200 \\
\hline $\mathrm{C}$ & -2.29517600 & 0.81292400 & 1.88532100 \\
\hline $\mathrm{O}$ & -3.00527700 & -0.03980100 & 1.26269800 \\
\hline $\mathrm{Rh}$ & -2.16686800 & -1.08481200 & -0.30813000 \\
\hline $\mathrm{O}$ & -1.50043300 & -2.52560300 & 1.01457000 \\
\hline $\mathrm{C}$ & -0.37313000 & -2.36582800 & 1.58383600 \\
\hline $\mathrm{O}$ & 0.41306600 & -1.37483400 & 1.41585600 \\
\hline $\mathrm{O}$ & -2.72141200 & 0.42204400 & -1.60925100 \\
\hline $\mathrm{C}$ & -1.91820700 & 1.39118000 & -1.79797500 \\
\hline $\mathrm{O}$ & -0.77849400 & 1.53289000 & -1.24374300 \\
\hline $\mathrm{O}$ & -1.22258900 & -2.07313200 & -1.85136900 \\
\hline $\mathrm{C}$ & -0.00107200 & -1.79861600 & -2.09056400 \\
\hline $\mathrm{O}$ & 0.70923700 & -0.95117200 & -1.45833900 \\
\hline $\mathrm{N}$ & 1.77287900 & 1.43449300 & 1.87415800 \\
\hline $\mathrm{N}$ & 1.49018400 & 1.51118900 & 2.96267700 \\
\hline $\mathrm{C}$ & 0.65895300 & -2.51202800 & -3.24336100 \\
\hline $\mathrm{H}$ & 0.45408400 & -1.95237800 & -4.16450300 \\
\hline $\mathrm{H}$ & 1.74059300 & -2.55212100 & -3.09582200 \\
\hline $\mathrm{H}$ & 0.24941500 & -3.51889100 & -3.35564800 \\
\hline $\mathrm{C}$ & -2.34932100 & 2.48637400 & -2.74044200 \\
\hline $\mathrm{H}$ & -2.64015900 & 3.36530500 & -2.15268500 \\
\hline $\mathrm{H}$ & -1.51328300 & 2.77319800 & -3.38454300 \\
\hline $\mathrm{H}$ & -3.19993000 & 2.16656400 & -3.34566500 \\
\hline $\mathrm{C}$ & -2.95237400 & 1.59055800 & 2.99704300 \\
\hline $\mathrm{H}$ & -2.23913300 & 1.77171500 & 3.80535200 \\
\hline $\mathrm{H}$ & -3.27243200 & 2.56271800 & 2.60209600 \\
\hline $\mathrm{H}$ & -3.82918500 & 1.05944300 & 3.37332700 \\
\hline $\mathrm{C}$ & 0.08999200 & -3.42943200 & 2.54645200 \\
\hline $\mathrm{H}$ & -0.62779400 & -4.24983200 & 2.59934600 \\
\hline $\mathrm{H}$ & 1.06603900 & -3.80734500 & 2.22429000 \\
\hline $\mathrm{H}$ & 0.21783900 & -2.98592000 & 3.54006300 \\
\hline $\mathrm{O}$ & 1.01530800 & 3.37946300 & 0.46886100 \\
\hline $\mathrm{C}$ & 0.50172800 & 4.50421800 & -0.27376200 \\
\hline $\mathrm{H}$ & -0.15816700 & 5.02746700 & 0.41870600 \\
\hline $\mathrm{H}$ & 1.31692500 & 5.15987900 & -0.59199300 \\
\hline $\mathrm{H}$ & -0.05616300 & 4.14877400 & -1.14261000 \\
\hline
\end{tabular}




\begin{tabular}{|c|c|c|c|}
\hline $\mathrm{Rh}$ & -0.10412100 & 0.07235300 & 0.04298800 \\
\hline $\mathrm{C}$ & 1.94489200 & 0.74249700 & 0.27081100 \\
\hline $\mathrm{C}$ & 2.04340400 & 2.11716900 & -0.30434500 \\
\hline $\mathrm{O}$ & 2.36439600 & 2.27999500 & -1.46730900 \\
\hline $\mathrm{C}$ & 3.09643600 & -0.16983600 & 0.09263100 \\
\hline $\mathrm{C}$ & 4.34641000 & 0.29597100 & -0.36847100 \\
\hline $\mathrm{C}$ & 5.40623600 & -0.58824800 & -0.55029600 \\
\hline $\mathrm{C}$ & 5.24748200 & -1.94549600 & -0.25169500 \\
\hline $\mathrm{C}$ & 4.02174900 & -2.41614900 & 0.22867100 \\
\hline $\mathrm{C}$ & 2.95288500 & -1.53959900 & 0.39532800 \\
\hline $\mathrm{H}$ & 2.00399700 & -1.90441000 & 0.76595000 \\
\hline $\mathrm{H}$ & 3.89704500 & -3.46860000 & 0.46761300 \\
\hline $\mathrm{H}$ & 6.07793700 & -2.63261900 & -0.38982700 \\
\hline $\mathrm{H}$ & 6.35945500 & -0.21789000 & -0.91669400 \\
\hline $\mathrm{H}$ & 4.48319100 & 1.34607700 & -0.59918700 \\
\hline $\mathrm{O}$ & -0.85638400 & 1.46646200 & 1.36331900 \\
\hline $\mathrm{C}$ & -2.11685100 & 1.52119700 & 1.56545600 \\
\hline $\mathrm{O}$ & -2.99298000 & 0.76958300 & 1.03746200 \\
\hline $\mathrm{Rh}$ & -2.39362800 & -0.72775600 & -0.26673800 \\
\hline $\mathrm{O}$ & -2.07751500 & -1.98949300 & 1.35047200 \\
\hline $\mathrm{C}$ & -0.95706800 & -1.97584000 & 1.94130500 \\
\hline $\mathrm{O}$ & 0.04774700 & -1.24874100 & 1.62474100 \\
\hline $\mathrm{O}$ & -2.55892300 & 0.59407900 & -1.84817600 \\
\hline $\mathrm{C}$ & -1.57609900 & 1.34847000 & -2.12567500 \\
\hline $\mathrm{O}$ & -0.45124500 & 1.37108600 & -1.52326700 \\
\hline $\mathrm{O}$ & -1.64114000 & -2.16784300 & -1.54649700 \\
\hline $\mathrm{C}$ & -0.39483500 & -2.17726500 & -1.79245400 \\
\hline $\mathrm{O}$ & 0.47586800 & -1.38516200 & -1.29901600 \\
\hline $\mathrm{N}$ & 1.91139600 & 1.02401400 & 2.01340400 \\
\hline $\mathrm{N}$ & 1.59998000 & 0.77948800 & 3.05066100 \\
\hline $\mathrm{C}$ & 0.12054200 & -3.19557100 & -2.78014500 \\
\hline $\mathrm{H}$ & 0.27729700 & -2.69908000 & -3.74557700 \\
\hline $\mathrm{H}$ & 1.08433400 & -3.58915400 & -2.44548300 \\
\hline $\mathrm{H}$ & -0.59814400 & -4.00696900 & -2.91258600 \\
\hline $\mathrm{C}$ & -1.72369100 & 2.30501100 & -3.28433800 \\
\hline $\mathrm{H}$ & -1.41038700 & 3.30819700 & -2.97883500 \\
\hline $\mathrm{H}$ & -1.06131700 & 1.98459900 & -4.09681200 \\
\hline $\mathrm{H}$ & -2.75426200 & 2.32679600 & -3.64375900 \\
\hline $\mathrm{C}$ & -2.58668500 & 2.60159200 & 2.50889000 \\
\hline $\mathrm{H}$ & -1.97722900 & 2.59240600 & 3.41772900 \\
\hline $\mathrm{H}$ & -2.45091400 & 3.57714700 & 2.02721700 \\
\hline $\mathrm{H}$ & -3.64030300 & 2.46777600 & 2.76109800 \\
\hline $\mathrm{C}$ & -0.76214000 & -2.88512100 & 3.12912700 \\
\hline $\mathrm{H}$ & -1.65363700 & -3.48824500 & 3.30991900 \\
\hline
\end{tabular}


$\mathrm{H}$

$\mathrm{H}$

$\mathrm{O}$

C

$\mathrm{H}$

$\mathrm{H}$

$\mathrm{H}$

\section{INT1}

$\mathrm{Rh}$

C

C

$\mathrm{O}$

C

C

C

C

C

C

$\mathrm{H}$

$\mathrm{H}$

$\mathrm{H}$

$\mathrm{H}$

$\mathrm{H}$

$\mathrm{O}$

C

$\mathrm{O}$

$\mathrm{Rh}$

$\mathrm{O}$

C

$\mathrm{O}$

$\mathrm{O}$

C

$\mathrm{O}$

$\mathrm{O}$

C

O

C

$\mathrm{H}$

$\mathrm{H}$

$\mathrm{H}$

C

$\mathrm{H}$

$\mathrm{H}$

$\begin{array}{rrr}0.09926600 & -3.53740200 & 2.95080600 \\ -0.54003700 & -2.27865300 & 4.01421700 \\ 1.64945600 & 3.09521100 & 0.52091000 \\ 1.52059100 & 4.40096300 & -0.08007000 \\ 1.21493000 & 5.06304100 & 0.73032000 \\ 2.47522200 & 4.72778900 & -0.50121900 \\ 0.75884800 & 4.37792400 & -0.86408100\end{array}$

0.00977900

0.06409600

0.10940200

$-1.92995100$

0.57544000

0.28668400

$-2.12087100$

2.03499000

0.46282400

$-2.05139500$

2.59040500

$-0.24798000$

1.54434300

$-3.08879800$

0.30122000

0.21110700

$-4.39191600$

$-0.49832400$

0.40815200

$-5.51913500$

$-1.86069000$

0.31870900

$-5.38308400$

$-2.42414200$

0.01314400

$-4.11765600$

$-1.63673000$

$-0.19467900$

$-2.98019600$

$-2.06454400$

$-0.09088800$

$-2.00131200$

$-4.02711600$

$-3.47887700$

$-0.25248600$

$-6.26969800$

$-2.48385500$

$-0.43623200$

$-6.50466500$

$-0.07149800$

$-0.06551100$

0.47755800

$-4.50134300$

1.35511500

0.64470200

0.43243300

1.82362500

$-0.88965000$

1.62310300

2.05623800

$-1.29317900$

2.61629900

2.37672900

1.27630800

$-1.18606000$

$-0.53609100$

$-0.21498500$

1.81603800

0.59477000

$-1.43185700$

$-1.43728700$

$-1.99873900$

$-2.33232000$

$-0.90523800$

$-1.67827300$

2.77352600

0.39614900

1.58654600

1.81881200

0.93191300

2.22700000

0.59093500

0.95753400

1.87270500

1.97379800

$-2.30608300$

0.78762300

0.80497700

$-2.51667800$

1.22776400

$-0.19713000$

$-1.72643700$

1.12013900

0.53686000

$-3.81021500$

1.95784600

$-3.58759500$

2.98663400

$-4.33654700$

1.47471200

$-0.29319900$

1.42522500

$-4.44435700$

1.96661400

2.13382300

1.60996900

3.53869500

1.80531100

2.65388300

3.49720600

1.57499800

1.11958900

4.34319900 
$\mathrm{H}$

C

$\mathrm{H}$

$\mathrm{H}$

$\mathrm{H}$

C

$\mathrm{H}$

$\mathrm{H}$

$\mathrm{H}$

$\mathrm{O}$

C

$\mathrm{H}$

$\mathrm{H}$

$\mathrm{H}$

\section{1-INT2}

$\mathrm{Rh}$

$\mathrm{Rh}$

C

C

$\mathrm{O}$

C

$\mathrm{H}$

$\mathrm{H}$

$\mathrm{H}$

$\mathrm{O}$

C

C

C

C

C

C

$\mathrm{H}$

$\mathrm{H}$

$\mathrm{H}$

$\mathrm{H}$

N

$\mathrm{H}$

$\mathrm{O}$

C

O

$\mathrm{O}$

C

$\mathrm{O}$

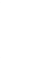

C

H

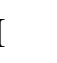

C

(

C

(

H

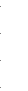

H

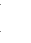

(

C

3.20336600

1.84551500

1.02388400

1.85300600

2.79916700

0.21004500

1.06248400

$-0.61356900$

$-0.14625800$

$-2.32438600$

$-2.26344700$

$-1.28248300$

$-2.41704600$

$-3.04708600$

1.56564500
3.40503600
3.63683400
4.16956900
3.43000400
-2.11802500
-2.64844600
-2.81536800
-1.36417300
2.64595700
4.08914200
4.41843300
4.39874900
4.50108100

3.75247300

$-1.93335000$

$-2.61713600$

$-1.14695300$

$-2.46433300$

$-3.62334600$

$-4.05181300$

$-3.44017200$

$-4.33463300$

$-0.71057000$

$-0.69253500$

$-0.33920000$

$-1.72657200$

$-0.05108500$

$-0.71690900$

0.02893300

0.04625200

$-3.11559700$

$-0.24861800$

$-0.38585100$

1.54076900

0.15426000

0.33978500

1.63167500

$-0.60389900$

1.61115000

1.51414600

0.18837400

2.69210000

1.39143400

$-0.49373600$

3.95162800

0.58579500

$-1.22836700$

3.91001300

1.16277600

0.28346700

4.68243700

2.33104700

$-0.99057100$

4.21420500

1.72809400

$-1.82467800$

1.68435900

2.18456000

1.51036100

0.26503100

1.72576700

2.47790000

$-0.64432700$

2.36181800

3.71583800

$-0.75678200$

3.46479100

4.01872600

0.04494200

3.93098900

3.06928100

0.95815100

3.30282600

1.82802300

1.05952700

3.68829600

1.09484000

1.75978100

4.79185500

3.28903800

1.58439800

3.95539000

4.98502000

$-0.03933600$

1.98628100

4.44796600

$-1.46731500$

2.10173600

$-0.79972900$

$-0.75398000$

0.86063400

2.26743700

$-1.25902900$

$-0.43909600$

$-1.90500200$

$-0.63151600$

$-1.46212600$

$-2.61002600$

$-0.95054500$

$-2.66508000$

$-2.22013000$

$-0.91315400$

$-2.76178500$

0.33095100

$-2.35615000$

$-1.59608800$

0.64023700

$-2.71456200$

$-0.56952000$

0.66789900

$-1.93292800$ 


\begin{tabular}{|c|c|c|c|}
\hline $\mathrm{O}$ & -3.33030100 & -0.81367300 & 1.58212100 \\
\hline $\mathrm{C}$ & -2.29603500 & -0.89950500 & 2.31477800 \\
\hline $\mathrm{O}$ & -1.09933300 & -0.64000100 & 1.96023800 \\
\hline $\mathrm{O}$ & -3.34534700 & 1.73421900 & 0.17073700 \\
\hline $\mathrm{C}$ & -2.33159600 & 2.38085700 & 0.58673300 \\
\hline $\mathrm{O}$ & -1.13358700 & 1.94695100 & 0.66030100 \\
\hline $\mathrm{C}$ & -1.33897800 & 1.00957000 & -4.15101000 \\
\hline $\mathrm{H}$ & -0.79175600 & 1.95662500 & -4.19564200 \\
\hline $\mathrm{H}$ & -0.70727700 & 0.23957800 & -4.60974300 \\
\hline $\mathrm{H}$ & -2.27550700 & 1.08926400 & -4.70538500 \\
\hline $\mathrm{C}$ & -2.56211500 & 3.79001900 & 1.07811700 \\
\hline $\mathrm{H}$ & -1.67311400 & 4.40324900 & 0.91180900 \\
\hline $\mathrm{H}$ & -3.43046800 & 4.23284300 & 0.58454300 \\
\hline $\mathrm{H}$ & -2.75794300 & 3.75485300 & 2.15718600 \\
\hline $\mathrm{C}$ & -2.47966800 & -1.37069300 & 3.73736500 \\
\hline $\mathrm{H}$ & -2.08145100 & -2.38805200 & 3.82937700 \\
\hline $\mathrm{H}$ & -1.91502500 & -0.72721700 & 4.41831900 \\
\hline $\mathrm{H}$ & -3.53644500 & -1.37407000 & 4.01169200 \\
\hline $\mathrm{C}$ & -1.17256100 & -4.01462400 & -1.41655300 \\
\hline $\mathrm{H}$ & -0.53244000 & -4.51930100 & -0.68580100 \\
\hline $\mathrm{H}$ & -2.09706200 & -4.57715800 & -1.55788200 \\
\hline $\mathrm{H}$ & -0.62388000 & -3.97084900 & -2.36453600 \\
\hline $\mathrm{H}$ & 1.54945300 & -1.66593000 & -0.64419200 \\
\hline $\mathrm{C}$ & 3.53939800 & -1.13248000 & -0.70107700 \\
\hline $\mathrm{C}$ & 3.91868300 & -2.38572700 & -0.22625700 \\
\hline $\mathrm{C}$ & 4.47360800 & -0.19405800 & -1.14025000 \\
\hline $\mathrm{C}$ & 5.27883700 & -2.69695300 & -0.16811500 \\
\hline $\mathrm{H}$ & 3.17017500 & -3.09592500 & 0.10381100 \\
\hline $\mathrm{C}$ & 5.82828400 & -0.52023600 & -1.07463500 \\
\hline $\mathrm{H}$ & 4.14822900 & 0.75860100 & -1.53655600 \\
\hline $\mathrm{C}$ & 6.23279800 & -1.76606200 & -0.58582900 \\
\hline $\mathrm{H}$ & 5.58733100 & -3.67104300 & 0.19977900 \\
\hline $\mathrm{H}$ & 6.56596300 & 0.20138300 & -1.41301800 \\
\hline $\mathrm{H}$ & 7.28956100 & -2.01365500 & -0.53945400 \\
\hline $\mathrm{O}$ & 1.87145200 & -0.31283000 & -2.06845000 \\
\hline $\mathrm{H}$ & 0.94065800 & 0.04285400 & -2.07451600 \\
\hline
\end{tabular}

\section{1-TS2}

$\begin{array}{lrrr}\mathrm{Rh} & -0.68789600 & -0.25875800 & 0.02351500 \\ \mathrm{Rh} & -2.79642900 & 0.84359500 & 0.32906200 \\ \mathrm{C} & 2.16090500 & -1.01273100 & -0.76879400 \\ \mathrm{C} & 1.49332200 & -1.86748200 & -1.77525600 \\ \mathrm{O} & 1.13192800 & -3.06589200 & -1.29865500 \\ \mathrm{C} & 0.40659800 & -3.90997000 & -2.21548400\end{array}$


H

$\mathrm{H}$

$\mathrm{H}$

O

C

C

C

C

C

C

$\mathrm{H}$

$\mathrm{H}$

$\mathrm{H}$

$\mathrm{H}$

$\mathrm{N}$

O

$\mathrm{H}$

O

C

O

O

C

O

O

C

O

O

C

O

C

$\mathrm{H}$

$\mathrm{H}$

$\mathrm{H}$

C

$\mathrm{H}$

$\mathrm{H}$

$\mathrm{H}$

C

$\mathrm{H}$

$\mathrm{H}$

$\mathrm{H}$

C

$\mathrm{H}$

H

\begin{tabular}{rrc}
-0.50511500 & -3.40483000 & -2.53989600 \\
0.16696000 & -4.81080900 & -1.64930200 \\
1.02769100 & -4.15925200 & -3.08078900 \\
1.30815400 & -1.50115600 & -2.93222300 \\
2.82284300 & -1.55728400 & 0.43405000 \\
2.10426600 & -2.23181200 & 1.43487600 \\
2.76646300 & -2.72506800 & 2.55929900 \\
4.14772800 & -2.55536100 & 2.69783800 \\
4.86916200 & -1.89655700 & 1.69711300 \\
4.21551500 & -1.40146700 & 0.56922100 \\
4.75881800 & -0.89661900 & -0.22545400 \\
5.94463000 & -1.77125900 & 1.79282300 \\
4.65823400 & -2.93810900 & 3.57765300 \\
2.19968000 & -3.23749000 & 3.33245500 \\
2.52551900 & 0.16442100 & -1.31158700 \\
4.17649500 & 0.04311100 & -2.38539000 \\
1.03127300 & -2.35400000 & 1.33978600 \\
-0.16709200 & 1.24815300 & -1.27824100 \\
-0.98516900 & 2.20373700 & -1.48754000 \\
-2.14734000 & 2.30667900 & -0.97660200 \\
-1.95788100 & 1.88764200 & 1.89804700 \\
-0.74425100 & 1.65720000 & 2.20761800 \\
0.01616500 & 0.81200700 & 1.63435100 \\
-3.52693300 & -0.24493200 & -1.25991400 \\
-2.75581900 & -1.06818000 & -1.85119500 \\
-1.53957900 & -1.29205000 & -1.54711100 \\
-3.32815500 & -0.67578500 & 1.62051000 \\
-2.49400500 & -1.61176800 & 1.84893300 \\
-1.33330300 & -1.71554600 & 1.33081400 \\
-0.14065800 & 2.47380100 & 3.32048500 \\
0.58351500 & 1.87618600 & 3.87962300 \\
0.38988300 & 3.32409900 & 2.87524900 \\
-0.91760400 & 2.85430800 & 3.98703800 \\
-2.89826100 & -2.66795900 & 2.84459400 \\
-2.38038200 & -3.60758300 & 2.63901000 \\
-2.61274000 & -2.32684500 & 3.84758000 \\
-3.98066600 & -2.81608800 & 2.82799000 \\
-3.32179500 & -1.86016000 & -3.00205200 \\
-2.64267600 & -1.79820800 & -3.85795700 \\
-3.39593700 & -2.91352100 & -2.70808600 \\
-4.31108800 & -1.49244900 & -3.28050000 \\
-0.51047100 & 3.31082100 & -2.39203400 \\
0.01148600 & 2.88942300 & -3.25576300 \\
-1.34544400 & 3.93348600 & -2.71879800 \\
& & \\
\hline
\end{tabular}


$\mathrm{H}$

C

C

C

C

C

C

$\mathrm{H}$

$\mathrm{H}$

$\mathrm{H}$

$\mathrm{H}$

$\mathrm{H}$

$\mathrm{H}$

$\mathrm{H}$

\section{1-INT3}

$\mathrm{Rh}$

$\mathrm{Rh}$

C

C

$\mathrm{O}$

C

$\mathrm{H}$

$\mathrm{H}$

$\mathrm{H}$

$\mathrm{O}$

C

C

C

C

C

C

$\mathrm{H}$

$\mathrm{H}$

$\mathrm{H}$

$\mathrm{H}$

$\mathrm{N}$

$\mathrm{O}$

$\mathrm{H}$

$\mathrm{O}$

C

$\mathrm{O}$

$\mathrm{O}$

C

C

0.20561800

2.77015800

2.83428900

3.13220500

3.36892600

3.31558100

3.01683300

3.20081700

3.60155700

3.50561100

2.96440300

2.03339300

2.49718000

3.92104400
3.92954800

1.54125400

1.39977200

2.49592900

3.74113100

3.89456000

2.79456800

2.34883200

4.58960200

4.86485700

2.90421600

0.31716000

0.71224500

$-0.78515700$
$-1.83832600$

0.74747500

$-0.63831500$

$-1.45727000$

$-0.88249100$

0.50787000

1.31291000

$-2.52883000$

$-1.51945100$

0.95811100

2.39196500

$-2.19355000$

1.38130800

$-2.83451900$

C

$-0.71108000$

$-0.13672600$

$-0.11539700$

$-2.84011700$

0.75296800

0.52419000

2.30380400

$-1.01622200$

$-0.90897600$

1.52320900

$-1.74923900$

$-1.93244300$

0.98722800

$-2.88536300$

$-1.47105300$

0.06465200

$-3.54959200$

$-2.35843300$

$-0.76086800$

$-2.87650300$

$-2.60059100$

$-0.29394400$

$-4.41679100$

$-1.80335700$

0.57333000

$-3.86665100$

$-3.27315700$

1.37914500

$-1.33100000$

$-3.07618400$

2.82499700

$-1.60538200$

0.33458400

4.19574200

$-1.43895500$

0.61950900

4.73682100

$-1.96475000$

1.79097400

3.92435100

$-2.66177400$

2.69129400

2.56747700

$-2.84211400$

2.40417100

2.01550900

$-2.32296900$

1.23342200

0.96179700

$-2.45909400$

1.02400200

1.93180900

$-3.38568200$

3.09857100

4.34670200

$-3.06742000$

3.60693400

5.79557400

$-1.83450200$

1.99949900

2.78366400

0.13606800

$-1.39109900$

4.59295300

$-0.06944700$

$-2.34235900$

4.81412500

$-0.91369700$

$-0.10482500$

$-1.51239400$

$-0.57884000$

$-1.95465800$

$-2.73869900$

$-0.31015300$

$-2.17740000$

$-3.53501400$

0.25005100

$-1.35620300$

$-2.21385100$

2.58218100

$-0.19707400$

$-1.04771500$

2.68048400

$-0.70128800$ 


$\begin{array}{lrrr}\mathrm{O} & -0.20230000 & 1.73274400 & -0.79684700 \\ \mathrm{O} & -3.34175800 & -1.12569400 & 1.21334200 \\ \mathrm{C} & -2.49523400 & -2.07168900 & 1.10545100 \\ \mathrm{O} & -1.33792700 & -1.97438500 & 0.58005000 \\ \mathrm{O} & -2.03734700 & 1.21139800 & 2.36420400 \\ \mathrm{C} & -0.82031200 & 0.91218400 & 2.59574900 \\ \mathrm{O} & -0.03700300 & 0.32494900 & 1.78148600 \\ \mathrm{C} & -0.63757200 & 4.01655900 & -1.26370900 \\ \mathrm{H} & 0.42891300 & 4.18446000 & -1.09317600 \\ \mathrm{H} & -0.81314300 & 4.00729400 & -2.34658800 \\ \mathrm{H} & -1.22781900 & 4.82091600 & -0.81972400 \\ \mathrm{C} & -0.25871900 & 1.31000700 & 3.93595300 \\ \mathrm{H} & 0.70824600 & 0.83316600 & 4.10740300 \\ \mathrm{H} & -0.13212500 & 2.39907800 & 3.95335500 \\ \mathrm{H} & -0.96043200 & 1.04269200 & 4.73136900 \\ \mathrm{C} & -2.88023800 & -3.41139400 & 1.67763400 \\ \mathrm{H} & -2.31241800 & -4.21138000 & 1.19739300 \\ \mathrm{H} & -2.64658900 & -3.41137300 & 2.74955600 \\ \mathrm{H} & -3.95358700 & -3.58175300 & 1.56287400 \\ \mathrm{C} & -3.29588200 & -0.72655800 & -3.51390600 \\ \mathrm{H} & -2.51095100 & -0.71544700 & -4.27379600 \\ \mathrm{H} & -3.67777600 & -1.75157900 & -3.42701400 \\ \mathrm{H} & -4.12220100 & -0.07476700 & -3.80683800 \\ \mathrm{C} & 2.66911100 & 1.49454200 & 0.66982200 \\ \mathrm{C} & 3.08861900 & 1.33500900 & -0.65245900 \\ \mathrm{C} & 3.71138100 & 2.38273600 & -1.33865200 \\ \mathrm{C} & 3.91646500 & 3.59908900 & -0.69000700 \\ \mathrm{C} & 3.50778500 & 3.76988100 & 0.63722600 \\ \mathrm{C} & 2.88560100 & 2.71539300 & 1.30942900 \\ \mathrm{H} & 4.04622800 & 2.21908800 & -2.35566000 \\ \mathrm{H} & 4.40230700 & 4.41231400 & -1.22160300 \\ \mathrm{H} & 3.67129300 & 4.71840200 & 1.14133100 \\ \mathrm{H} & 2.54897100 & 2.83987600 & 2.33502300 \\ \mathrm{H} & 2.46063500 & 0.31228300 & -2.34382600 \\ & 2.14438600 & 0.70508900 & 1.18706700 \\ \mathrm{H} & -0.97473300 & -2.64755300\end{array}$

\section{1-TS3}

$\begin{array}{lrrr}\mathrm{Rh} & -0.71848000 & -0.13147500 & -0.10210300 \\ \mathrm{Rh} & -2.85146100 & 0.78928700 & 0.47200200 \\ \mathrm{C} & 2.28886400 & -1.05764800 & -0.84377300 \\ \mathrm{C} & 1.53798900 & -1.84873900 & -1.85743300 \\ \mathrm{O} & 0.98012000 & -2.95003400 & -1.34631700 \\ \mathrm{C} & 0.09052800 & -3.66882000 & -2.22664300\end{array}$


$\mathrm{H}$

$\mathrm{H}$

$\mathrm{H}$

O

C

C

C

C

C

C

$\mathrm{H}$

$\mathrm{H}$

$\mathrm{H}$

$\mathrm{H}$

$\mathrm{N}$

O

$\mathrm{H}$

O

C

O

$\mathrm{O}$

C

O

O

C

O

O

C

O

C

$\mathrm{H}$

$\mathrm{H}$

$\mathrm{H}$

C

$\mathrm{H}$

$\mathrm{H}$

$\mathrm{H}$

C

$\mathrm{H}$

$\mathrm{H}$

$\mathrm{H}$

C

$\mathrm{H}$

$\mathrm{H}$

\begin{tabular}{rrr}
-0.73524100 & -3.01707100 & -2.52173000 \\
-0.27362600 & -4.51320300 & -1.64128300 \\
0.62857900 & -4.01990600 & -3.11129900 \\
1.44712200 & -1.49822700 & -3.02510700 \\
2.79412000 & -1.59610100 & 0.42762900 \\
4.16805600 & -1.42280700 & 0.69243200 \\
4.71537400 & -1.90378100 & 1.87910500 \\
3.90298900 & -2.55715000 & 2.81257400 \\
2.54326400 & -2.74451900 & 2.54372400 \\
1.98539000 & -2.27443400 & 1.35516600 \\
0.93054100 & -2.41551300 & 1.15433400 \\
1.91158300 & -3.25654200 & 3.26492700 \\
4.32930900 & -2.92563900 & 3.74185800 \\
5.77615800 & -1.77305600 & 2.07524600 \\
2.73996300 & 0.08576200 & -1.34040000 \\
4.55950800 & -0.15599800 & -2.56063600 \\
4.78326500 & -0.93330800 & -0.05960600 \\
-1.50512200 & -0.67916500 & -1.91866800 \\
-2.72834200 & -0.41899100 & -2.16853100 \\
-3.53058100 & 0.18755700 & -1.38675200 \\
-2.21698900 & 2.58092500 & -0.32542600 \\
-1.04761400 & 2.65370100 & -0.82706200 \\
-0.20565200 & 1.69923500 & -0.87882100 \\
-3.36275000 & -1.05586700 & 1.24103000 \\
-2.51611900 & -2.00531400 & 1.18786800 \\
-1.34570700 & -1.92622400 & 0.68754100 \\
-2.06443700 & 1.33586300 & 2.29366300 \\
-0.84762200 & 1.05696700 & 2.54916400 \\
-0.05308300 & 0.43904400 & 1.76889300 \\
-0.62033100 & 3.96408700 & -1.43481800 \\
0.40818900 & 4.19059800 & -1.13930800 \\
-0.64155200 & 3.86775100 & -2.52699400 \\
-1.29029500 & 4.77170000 & -1.13385700 \\
-0.30036600 & 1.52090000 & 3.87357500 \\
0.64873000 & 1.02834700 & 4.09379600 \\
-0.13990800 & 2.60472700 & 3.82571600 \\
-1.02470900 & 1.32423100 & 4.66901900 \\
-2.91024500 & -3.33364200 & 1.77994900 \\
-2.61609300 & -4.14253000 & 1.10467600 \\
-2.37029600 & -3.47317200 & 2.72402300 \\
-3.98408800 & -3.37231800 & 1.97195900 \\
-3.27105800 & -0.90454400 & -3.48756500 \\
-2.48235300 & -0.90897400 & -4.24387600 \\
-3.63040300 & -1.93329000 & -3.35916700 \\
& & \\
\hline
\end{tabular}


$\mathrm{H}$

C

C

C

C

C

C

$\mathrm{H}$

$\mathrm{H}$

$\mathrm{H}$

$\mathrm{H}$

$\mathrm{H}$

$\mathrm{H}$

$\mathrm{H}$

\section{2-TS3_0H $2 \mathrm{O}$}

$\mathrm{C}$

C

$\mathrm{O}$

C

$\mathrm{H}$

$\mathrm{H}$

$\mathrm{H}$

$\mathrm{O}$

C

C

C

C

C

C

$\mathrm{H}$

$\mathrm{H}$

$\mathrm{H}$

$\mathrm{H}$

$\mathrm{N}$

$\mathrm{O}$

$\mathrm{H}$

C

C

C

C

C

C

$\mathrm{H}$

-4.10910600
2.67700700
3.10109200
3.77911600
4.02736500
3.61126600
2.93705700
4.12118900
4.55525700
3.80950100
2.59525100
2.64404400
2.11359800
4.38394700

$-0.28380300$

1.51724100

1.28506500

2.27914400

3.50983800

3.75179800

2.75156600

2.05890100

4.27946000

4.71179300

2.93086600

0.18445100

0.77268800

$-1.09883700$

$-3.81223300$

0.66002600

$-0.65300200$

$-1.37013200$

$-0.76572400$

0.54786000

1.25333600

$-2.37374900$

$-1.32170000$

1.01643300

2.26901800

$-2.35589900$

1.20261000

$-2.73711900$

$\begin{array}{rrr}0.95333600 & -0.39202300 & -0.71090400 \\ 1.90746500 & -1.22148800 & -0.01511600 \\ 3.12204300 & -0.64573900 & 0.11590300 \\ 4.12665700 & -1.43704300 & 0.77531700 \\ 4.32398000 & -2.35849400 & 0.21953700 \\ 5.02051400 & -0.81166800 & 0.79645700 \\ 3.81957500 & -1.68992800 & 1.79465800 \\ 1.69109200 & -2.38130200 & 0.38039000 \\ 0.78225600 & 1.06363700 & -0.46425500 \\ 0.07873700 & 1.87636600 & -1.37587500 \\ -0.12589200 & 3.23239600 & -1.12570200 \\ 0.39224100 & 3.82303900 & 0.03048800 \\ 1.10397500 & 3.03473000 & 0.93789200 \\ 1.28187200 & 1.67075700 & 0.70356300 \\ 1.81993400 & 1.07114100 & 1.43018500 \\ 1.50971500 & 3.47755300 & 1.84411200 \\ 0.24465400 & 4.88275400 & 0.22016400 \\ -0.67784200 & 3.83181400 & -1.84514500 \\ -0.38701000 & -1.11546600 & -1.01948100 \\ -0.22127200 & -2.48110800 & -1.35511400 \\ -0.31461800 & 1.44376100 & -2.29353200 \\ -1.46388300 & -0.60534600 & 1.13458300 \\ -1.57150300 & -0.85594600 & -0.23439200 \\ -2.80999700 & -0.85784900 & -0.87828400 \\ -3.96359100 & -0.61285200 & -0.13274800 \\ -3.87120100 & -0.36002500 & 1.23996200 \\ -2.62314600 & -0.35725300 & 1.87078300 \\ -2.85820200 & -1.05095300 & -1.94572900\end{array}$


$\mathrm{H}$

$\mathrm{H}$

$\mathrm{H}$

$\mathrm{H}$

$\mathrm{H}$

$\mathrm{H}$

\section{2-TS3_1 $\mathrm{H}_{2} \mathrm{O}$}

$\mathrm{C}$

C

$\mathrm{O}$

C

$\mathrm{H}$

$\mathrm{H}$

$\mathrm{H}$

$\mathrm{O}$

C

C

C

C

C

C

$\mathrm{H}$

$\mathrm{H}$

$\mathrm{H}$

$\mathrm{H}$

$\mathrm{N}$

$\mathrm{O}$

$\mathrm{H}$

C

C

C

C

C

C

$\mathrm{H}$

$\mathrm{H}$

$\mathrm{H}$

$\mathrm{H}$

$\mathrm{H}$

$\mathrm{H}$

$\mathrm{H}$

$\mathrm{O}$

$\mathrm{H}$
$-4.93239400$

$-0.61172600$

$-0.62426200$

$-4.77032100$

$-0.16037200$

1.81629100

$-2.55056300$

$-0.16078600$

2.93679700

0.28774800

$-0.57925000$

$-1.87060700$

$-0.48810900$

$-0.60338200$

$-2.81361800$

1.60903600

$-0.61289900$

$-1.07819000$

$-0.11131500$

0.61634900

$-2.02418500$

$-0.75720400$

$-0.30272700$

$-2.90002700$

0.10960200

$-0.82101500$

$-3.92802200$

$-0.44639300$

$-1.66834000$

$-4.53943100$

$-1.16241600$

$-1.11262800$

$-4.53108000$

0.40557800

$-1.98344700$

$-3.48433800$

$-0.94082000$

$-2.53688000$

$-2.09367300$

$-1.97404200$

$-0.53925300$

$-0.47615500$

1.20509600

0.19232900

$-0.10104400$

2.14746800

1.16395800

0.50959400

3.34698700

0.79005100

0.74762600

3.62689200

$-0.55728900$

0.37404400

2.69731600

$-1.53280700$

$-0.22406100$

1.49437400

$-1.15968400$

$-0.49846200$

0.77384300

$-1.92441600$

0.55756400

2.90491500

$-2.58379600$

1.21981000

4.56203900

$-0.84696600$

0.79459400

4.06505900

1.55465400

0.04962200

$-1.06586500$

1.08566000

$-0.42619400$

$-2.38142300$

1.37890000

$-0.28712400$

1.92948200

2.21073700

1.27606400

$-1.70906300$

$-0.95642900$

1.28327600

$-1.11522600$

0.30586900

2.43060400

$-0.54018500$

0.84335500

3.60851900

$-0.55851000$

0.09128100

3.62307900

$-1.15029600$

$-1.17321800$

2.45805000

$-1.72479100$

$-1.69493700$

2.40104100

$-0.07890700$

1.82574800

4.51068500

$-0.11116200$

0.49787200

4.54056000

$-1.16422800$

$-1.75460000$

2.46907700

$-2.18412400$

$-2.67899300$

0.12532300

$-0.66032000$

2.08919500

0.36312300

$-2.14233000$

$-1.35147600$

$-1.05558800$

$-2.55525400$

0.62300700

$-0.95642700$

0.01356600

3.13307500

$-1.35798700$

$-0.77422000$

3.53887400 


\section{2-TS3_2 $\mathrm{H}_{2} \mathrm{O}$}

$\mathrm{C}$

$\mathrm{C}$

$\mathrm{O}$

$\mathrm{C}$

$\mathrm{H}$

$\mathrm{H}$

$\mathrm{H}$

$\mathrm{O}$

$\mathrm{C}$

$\mathrm{C}$

$\mathrm{C}$

$\mathrm{C}$

$\mathrm{C}$

$\mathrm{C}$

$\mathrm{H}$

$\mathrm{H}$

$\mathrm{H}$

$\mathrm{H}$

$\mathrm{N}$

$\mathrm{O}$

$\mathrm{H}$

C

C

C

C

C

C

$\mathrm{H}$

$\mathrm{H}$

$\mathrm{H}$

$\mathrm{H}$

$\mathrm{H}$

$\mathrm{H}$

$\mathrm{H}$

$\mathrm{O}$

$\mathrm{H}$

$\mathrm{H}$

$\mathrm{O}$

$\mathrm{H}$

$\mathrm{H}$

$\begin{array}{rrr}-0.95107000 & -0.43794400 & 0.29069700 \\ -1.18661400 & -1.60682900 & -0.57477100 \\ -1.99030100 & -1.33900600 & -1.60587800 \\ -2.40773500 & -2.46906400 & -2.40271500 \\ -2.93168300 & -3.20276000 & -1.78426900 \\ -3.07900000 & -2.05599500 & -3.15601300 \\ -1.54424700 & -2.94099900 & -2.87927200 \\ -0.79260800 & -2.75732700 & -0.32957900 \\ -0.90888000 & 0.92480300 & -0.35261600 \\ -1.57252000 & 2.00144700 & 0.25588700 \\ -1.50784900 & 3.28120400 & -0.30148300 \\ -0.78449700 & 3.50008100 & -1.47590500 \\ -0.13002100 & 2.43146000 & -2.09724600 \\ -0.18966000 & 1.15593300 & -1.53727900 \\ 0.32702000 & 0.32996600 & -2.01718500 \\ 0.43023300 & 2.59302200 & -3.01440600 \\ -0.73583500 & 4.49549900 & -1.90958000 \\ -2.02979200 & 4.10436500 & 0.17937400 \\ 0.28602900 & -0.64287100 & 1.18033500 \\ 0.20658800 & -1.86798000 & 1.91041200 \\ -2.15899000 & 1.82770700 & 1.15184800 \\ 2.13314500 & -1.45269300 & -0.28508300 \\ 1.60683600 & -0.47148100 & 0.55601900 \\ 2.28610700 & 0.71853600 & 0.80764600 \\ 3.52043300 & 0.93791600 & 0.19168000 \\ 4.06060100 & -0.02916400 & -0.65774000 \\ 3.36814600 & -1.22208600 & -0.89095600 \\ 1.85619100 & 1.46716000 & 1.46544000 \\ 4.05496500 & 1.86432700 & 0.37971700 \\ 5.02046500 & 0.14286900 & -1.13639300 \\ 3.78936200 & -1.97822000 & -1.54698900 \\ 0.15835400 & 0.08132300 & 1.97038200 \\ 1.59265500 & -2.37354900 & -0.46684700 \\ -0.09484300 & -2.50832400 & 1.20825000 \\ -2.54741800 & -0.17235600 & 2.35654400 \\ -2.56504500 & -1.04569700 & 2.78537200 \\ -1.85474000 & -0.41477300 & 1.22027200 \\ -0.42438100 & 0.91074800 & 3.19492200 \\ -1.37595000 & 0.50403900 & 2.98571400 \\ -0.49488900 & 1.84479200 & 2.93407000\end{array}$




\begin{tabular}{|c|c|c|c|}
\hline \multicolumn{4}{|c|}{ 2-TS3 } \\
\hline $\mathrm{C}$ & -0.62225500 & -0.75126400 & -0.05045200 \\
\hline $\mathrm{C}$ & -0.12188900 & -2.11413900 & 0.24209700 \\
\hline $\mathrm{O}$ & -0.45054600 & -2.54345000 & 1.45845300 \\
\hline $\mathrm{C}$ & -0.16900300 & -3.93224700 & 1.74587100 \\
\hline $\mathrm{H}$ & -0.69638600 & -4.58135600 & 1.04210400 \\
\hline $\mathrm{H}$ & -0.53169000 & -4.09317300 & 2.76102600 \\
\hline $\mathrm{H}$ & 0.90610100 & -4.12184400 & 1.68978900 \\
\hline $\mathrm{O}$ & 0.42452500 & -2.84569900 & -0.59434200 \\
\hline $\mathrm{C}$ & -0.73214800 & 0.24356500 & 1.07266000 \\
\hline $\mathrm{C}$ & -1.79612300 & 1.15649400 & 1.06770900 \\
\hline $\mathrm{C}$ & -1.90473900 & 2.12528000 & 2.06742300 \\
\hline $\mathrm{C}$ & -0.95338800 & 2.18839100 & 3.08806400 \\
\hline $\mathrm{C}$ & 0.11207300 & 1.28235000 & 3.10131200 \\
\hline $\mathrm{C}$ & 0.22474900 & 0.32091900 & 2.09762600 \\
\hline $\mathrm{H}$ & 1.05775900 & -0.37553800 & 2.10669000 \\
\hline $\mathrm{H}$ & 0.85843400 & 1.32895500 & 3.88997600 \\
\hline $\mathrm{H}$ & -1.03887600 & 2.94037000 & 3.86816100 \\
\hline $\mathrm{H}$ & -2.73408500 & 2.82743300 & 2.04939000 \\
\hline $\mathrm{N}$ & 0.14744500 & -0.12780300 & -1.22216200 \\
\hline $\mathrm{O}$ & 0.09853200 & -0.95906000 & -2.38384900 \\
\hline $\mathrm{H}$ & -2.52989300 & 1.11430000 & 0.27046700 \\
\hline $\mathrm{C}$ & 2.58287400 & -0.56373800 & -0.87867200 \\
\hline $\mathrm{C}$ & 1.52015100 & 0.33787300 & -0.94277900 \\
\hline $\mathrm{C}$ & 1.69938300 & 1.70167500 & -0.71476200 \\
\hline $\mathrm{C}$ & 2.97808400 & 2.17158300 & -0.40607000 \\
\hline $\mathrm{C}$ & 4.05434200 & 1.28544900 & -0.33449500 \\
\hline $\mathrm{C}$ & 3.85443100 & -0.07792100 & -0.57349000 \\
\hline $\mathrm{H}$ & 0.85546400 & 2.38042500 & -0.77437800 \\
\hline $\mathrm{H}$ & 3.12667100 & 3.23181200 & -0.22396400 \\
\hline $\mathrm{H}$ & 5.04792300 & 1.65405600 & -0.09596600 \\
\hline $\mathrm{H}$ & 4.69007300 & -0.76989900 & -0.52430400 \\
\hline $\mathrm{H}$ & -0.43095100 & 0.72098100 & -1.53841900 \\
\hline $\mathrm{H}$ & 2.42673000 & -1.61928300 & -1.06116000 \\
\hline $\mathrm{H}$ & 0.30294600 & -1.85732500 & -2.00429300 \\
\hline $\mathrm{O}$ & -2.90709200 & -1.07233100 & -1.36060000 \\
\hline $\mathrm{H}$ & -2.49314600 & -1.35525200 & -2.19437600 \\
\hline $\mathrm{H}$ & -1.73796500 & -0.93716000 & -0.58620600 \\
\hline $\mathrm{O}$ & -3.67071000 & 1.22403600 & -1.99036500 \\
\hline $\mathrm{H}$ & -3.41506100 & 0.22006600 & -1.70839500 \\
\hline $\mathrm{H}$ & -4.18389200 & 1.58367900 & -1.24828400 \\
\hline $\mathrm{O}$ & -1.20316900 & 2.00719100 & -2.07850300 \\
\hline $\mathrm{H}$ & -1.00404100 & 2.08258500 & -3.02817800 \\
\hline $\mathrm{H}$ & -2.19980800 & 1.78966600 & -2.03314000 \\
\hline
\end{tabular}




\section{2-INT4}

C

C

$\mathrm{O}$

C

$\mathrm{H}$

$\mathrm{H}$

$\mathrm{H}$

$\mathrm{O}$

C

C

C

C

C

C

$\mathrm{H}$

$\mathrm{H}$

$\mathrm{H}$

$\mathrm{H}$

$\mathrm{N}$

$\mathrm{O}$

$\mathrm{H}$

C

C

C

C

C

C

$\mathrm{H}$

$\mathrm{H}$

$\mathrm{H}$

$\mathrm{H}$

$\mathrm{H}$

$\mathrm{H}$

$\mathrm{H}$
$-0.67963700$

$-2.02314900$

$-2.88605500$

$-4.21388400$

$-4.17141300$

$-4.77019000$

$-4.67654100$

$-2.28999800$

$-0.76552900$

$-0.09236500$

$-0.10117900$

$-0.78550700$

$-1.46052400$

$-1.44953200$

$-1.96913700$

$-1.99507700$

$-0.79486500$

0.42454200

0.34460600

0.06501400

0.44438900

2.53369600

1.69290500

2.19903100

3.51736200

4.35852400

3.85888800

1.56558100

3.89176900

5.38851100

4.49935000

2.15082400

$-0.63479300$

$-0.41147100$
$-0.64273500$

$-1.31086700$

$-1.08095900$

$-1.62658700$

$-2.71753300$

$-1.31490000$

$-1.22557800$

$-1.95615400$

0.87311400

1.66493900

3.05711100

3.67091800

2.88643400

1.49500200

0.89090600

3.35792300

4.75428200

3.66003900

$-1.25474300$

$-0.88636200$

1.18880700

$-0.24893900$

$-0.92791600$

$-1.33783300$

$-1.05325100$

$-0.37451600$

0.01640400

$-1.89353600$

$-1.37785600$

$-0.16158000$

0.54282900

0.06605600

$-1.53026700$

$-0.91627600$
$-0.32422500$

$-0.03563800$

$-1.02785600$

$-0.85735900$

$-0.79693400$

$-1.74156400$

0.04845100

0.96368300

$-0.22587200$

$-1.16539900$

$-1.05464100$

$-0.00232000$

0.93763900

0.82815100

1.56527300

1.75812700

0.08414600

$-1.79039300$

0.56134600

1.91678200

$-1.98162300$

1.13173000

0.23954000

$-1.00703200$

$-1.35637100$

$-0.46772500$

0.77533500

$-1.69220800$

$-2.32388400$

$-0.74003300$

1.47867900

2.09384200

2.14003000

$-1.34708400$

\section{2-TS4_0H2O}

$\mathrm{C}$

C

$\mathrm{O}$

C

$\mathrm{H}$

$\mathrm{H}$
0.60480200

0.14860500

$-1.01251300$

$-1.58579000$

$-1.78633500$

$-2.51901100$
$-0.25199100$

$-1.58417900$

$-1.96666000$

$-3.18840500$

$-3.10896100$

$-3.31745300$
0.42715100

$-0.11148500$

0.45405800

$-0.04628800$

$-1.11895800$

0.50345400 
$\mathrm{H}$

$\mathrm{O}$

C

C

C

C

C

C

$\mathrm{H}$

$\mathrm{H}$

$\mathrm{H}$

$\mathrm{H}$

$\mathrm{N}$

$\mathrm{O}$

$\mathrm{H}$

C

C

C

C

C

C

$\mathrm{H}$

$\mathrm{H}$

$\mathrm{H}$

$\mathrm{H}$

$\mathrm{H}$

$\mathrm{H}$

$\mathrm{H}$

\section{2-TS4_1 $1 \mathrm{H}_{2} \mathrm{O}$}

C

C

$\mathrm{O}$

C

$\mathrm{H}$

$\mathrm{H}$

$\mathrm{H}$

$\mathrm{O}$

C

C

C

C

C

C

$\begin{array}{rrr}-0.91468900 & -4.03261600 & 0.13647900 \\ 0.76224700 & -2.26584700 & -0.91178700 \\ 1.99394400 & 0.19553300 & 0.07988200 \\ 2.24922300 & 1.50628100 & -0.35125500 \\ 3.55139400 & 1.92076200 & -0.63930900 \\ 4.61979700 & 1.02994700 & -0.51022200 \\ 4.37425200 & -0.27923000 & -0.08398300 \\ 3.07555600 & -0.69123900 & 0.21375500 \\ 2.89520400 & -1.70847500 & 0.54680400 \\ 5.19722700 & -0.98139200 & 0.02306700 \\ 5.63252200 & 1.35061200 & -0.73986600 \\ 3.72788100 & 2.94009900 & -0.97362600 \\ -0.31250000 & 0.77472900 & 0.66663400 \\ -0.41343100 & 0.33888600 & 2.49282100 \\ 1.42250100 & 2.20182900 & -0.46074400 \\ -2.69531100 & 1.25873500 & 0.95628900 \\ -1.62898700 & 0.78355100 & 0.17704700 \\ -1.83652600 & 0.48281400 & -1.18361100 \\ -3.09575800 & 0.68136000 & -1.74979700 \\ -4.16042000 & 1.14295500 & -0.97294900 \\ -3.95281400 & 1.42308400 & 0.38280200 \\ -1.01114100 & 0.13528900 & -1.79668800 \\ -3.24164100 & 0.45868300 & -2.80322900 \\ -5.14338400 & 1.27598800 & -1.41540300 \\ -4.77749400 & 1.76889600 & 1.00006300 \\ -2.52589500 & 1.44728400 & 2.01043900 \\ 0.10773000 & 1.08477200 & 2.85766100 \\ 0.46178200 & -0.36855800 & 1.75520100\end{array}$

$\begin{array}{rrr}0.56336900 & 0.39286900 & 0.20709200 \\ 0.05572800 & 1.38725100 & -0.77290700 \\ -1.08175000 & 1.97380900 & -0.32036200 \\ -1.72106200 & 2.87770100 & -1.23379900 \\ -2.00569900 & 2.35983000 & -2.15511700 \\ -2.61245400 & 3.23583100 & -0.71616100 \\ -1.06250600 & 3.71658500 & -1.47886300 \\ 0.58441300 & 1.70343900 & -1.82778400 \\ 1.89167000 & -0.25416100 & -0.04104500 \\ 2.09327100 & -1.62271800 & 0.20914500 \\ 3.34133900 & -2.21517000 & 0.00686900 \\ 4.41933800 & -1.45547200 & -0.45502300 \\ 4.23282000 & -0.09248400 & -0.70630800 \\ 2.98876800 & 0.50250600 & -0.49563200\end{array}$




$\begin{array}{lrrc}\mathrm{H} & 2.85889000 & 1.56160200 & -0.69343900 \\ \mathrm{H} & 5.06220400 & 0.51427500 & -1.06189000 \\ \mathrm{H} & 5.38982200 & -1.91754900 & -0.61592700 \\ \mathrm{H} & 3.46769700 & -3.27695300 & 0.20520800 \\ \mathrm{~N} & -0.35825200 & -0.38033700 & 0.90667700 \\ \mathrm{O} & -0.68457200 & 0.21984600 & 2.46332100 \\ \mathrm{H} & 1.26029700 & -2.22155900 & 0.56416700 \\ \mathrm{C} & -2.80653100 & -0.79690800 & 1.11189600 \\ \mathrm{C} & -1.62207500 & -0.72081300 & 0.36422800 \\ \mathrm{C} & -1.64204900 & -1.12328900 & -0.98811000 \\ \mathrm{C} & -2.82216100 & -1.58731100 & -1.56583800 \\ \mathrm{C} & -4.00416300 & -1.65604100 & -0.82304500 \\ \mathrm{C} & -3.98214000 & -1.25689600 & 0.51453300 \\ \mathrm{H} & -0.73116900 & -1.08542500 & -1.57637600 \\ \mathrm{H} & -2.81176900 & -1.89246500 & -2.60913700 \\ \mathrm{H} & -4.92306000 & -2.01395600 & -1.27870400 \\ \mathrm{H} & -4.89135200 & -1.29648600 & 1.10944500 \\ \mathrm{H} & -2.80811000 & -0.47906600 & 2.14528900 \\ \mathrm{H} & -0.32031800 & -0.53024000 & 2.97148100 \\ \mathrm{H} & 0.95408600 & 1.45295900 & 1.23601900 \\ \mathrm{O} & 0.91302100 & 2.00135600 & 2.22946000 \\ \mathrm{H} & 1.75725400 & 1.80766100 & 2.68908700 \\ \mathrm{H} & 0.15833100 & 1.26531700 & 2.56875200\end{array}$

\section{2-TS4}

$\mathrm{C}$

C

$\mathrm{O}$

C

$\mathrm{H}$

$\mathrm{H}$

$\mathrm{H}$

$\mathrm{O}$

C

C

C

C

C

C

$\mathrm{H}$

$\mathrm{H}$

$\mathrm{H}$

$\mathrm{H}$

$\mathrm{N}$
$-0.50062200$

$-0.16633500$

1.05370300

1.43491500

1.55803600

2.39072200

0.69443600

$-0.84654500$

$-1.74296500$

$-1.94675700$

$-3.12030200$

$-4.14986100$

$-3.97013700$

$-2.79884800$

$-2.68222100$

$-4.75309200$

$-5.06524400$

$-3.22934100$

0.50282200
0.13905700

1.11048000

0.10849200

1.70485000

1.11737600

2.74539500

0.85572500

2.36570000

1.75589900

2.77604600

3.12255600

1.38488600

3.55234100

1.76781100

1.48252500

2.07880500

$-0.65073800$

0.09629500

$-1.64214800$

$-0.89704100$

$-2.38967700$

$-0.95705700$

$-2.18633100$

$-0.03130400$

$-1.21258200$

0.95532700

$-0.45733100$

1.02376000

0.29010800

1.79792800

$-1.03423300$

1.68992500

$-2.77028000$

$-0.07884800$

$-3.14240600$

$-1.73547700$

$-0.26198700$

$-0.79373100$ 
O

$\mathrm{H}$

C

C

C

C

C

C

$\mathrm{H}$

$\mathrm{H}$

$\mathrm{H}$

$\mathrm{H}$

$\mathrm{H}$

$\mathrm{H}$

$\mathrm{H}$

$\mathrm{O}$

$\mathrm{H}$

$\mathrm{H}$

$\mathrm{O}$

$\mathrm{H}$

$\mathrm{H}$

\section{3-TS2_0 $\mathrm{H}_{2} \mathrm{O}$}

$\mathrm{Rh}$

$\mathrm{Rh}$

C

C

O

C

$\mathrm{H}$

$\mathrm{H}$

$\mathrm{H}$

$\mathrm{O}$

C

C

C

C

C

C

$\mathrm{H}$

$\mathrm{H}$

$\mathrm{H}$

$\mathrm{H}$

$\mathrm{N}$

$\begin{array}{rrr}0.75510800 & 0.70795500 & -1.91798400 \\ -1.15949900 & -1.82404300 & -1.62264100 \\ 2.87890900 & -0.78958200 & -1.19775000 \\ 1.73444900 & -0.80662000 & -0.38035500 \\ 1.79809800 & -1.47458300 & 0.85896800 \\ 2.97487800 & -2.10420900 & 1.25979600 \\ 4.11470000 & -2.08240700 & 0.45007200 \\ 4.05185200 & -1.41914400 & -0.77739700 \\ 0.92189200 & -1.49862500 & 1.49722900 \\ 2.99762000 & -2.61181000 & 2.22115100 \\ 5.03079600 & -2.57091200 & 0.77011600 \\ 4.92583100 & -1.38620800 & -1.42383100 \\ 2.84892700 & -0.27809100 & -2.15124900 \\ 0.16051600 & 0.38169600 & -2.61995100 \\ -1.75887200 & 1.57012900 & -0.55842600 \\ 0.19954200 & 3.16012900 & -1.52866000 \\ 0.27618800 & 3.60416700 & -2.40047400 \\ 0.56500900 & 2.18464900 & -1.62456200 \\ -2.07357900 & 2.44385900 & -0.92018600 \\ -0.85391600 & 2.98259900 & -1.30420900 \\ -2.64522800 & 2.22453800 & -1.68139900\end{array}$

$-0.69559400$

$-0.11889100$

0.04573800

$-3.04829000$

0.05084200

$-0.57515900$

1.67172300

$-0.15195700$

0.51888300

1.47834800

$-0.88208600$

1.74669100

1.15837800

$-0.28719800$

2.86986300

0.84731500

$-1.13182600$

4.00748700

0.08008700

$-1.85374700$

3.73191500

0.47881400

$-0.44730700$

4.77111600

1.75247300

$-1.63906800$

4.35122200

1.63531400

$-2.15815800$

1.68931300

1.26248600

0.38222000

2.45952600

1.73788700

$-0.89034000$

2.84590000

3.06373900

$-1.07566900$

3.95530300

0.00100600

2.48367200

3.49700900

1.26617500

2.16831600

1.45894000

1.83936200

2.44770700

1.81121500

4.17603000

2.11552700

4.99056200

$-0.14382900$

3.15697900

3.40132700

$-2.06877100$

2.35144800

$-1.17172500$

$-0.34445000$ 


$\begin{array}{lrrr}\mathrm{H} & 2.42252200 & 1.06513000 & -1.73759600 \\ \mathrm{O} & -0.65732800 & -1.94615200 & -0.94153300 \\ \mathrm{C} & -1.71678100 & -2.37267300 & -1.53525700 \\ \mathrm{O} & -2.83944700 & -1.79582200 & -1.51796900 \\ \mathrm{O} & -2.45201200 & 0.99827300 & -2.31184200 \\ \mathrm{C} & -1.21026600 & 1.19640700 & -2.50290900 \\ \mathrm{O} & -0.26676500 & 0.84053800 & -1.72528800 \\ \mathrm{O} & -3.51561500 & -0.89412800 & 1.20126600 \\ \mathrm{C} & -2.57425200 & -1.26121800 & 1.97157300 \\ \mathrm{O} & -1.32795300 & -1.09214400 & 1.76343300 \\ \mathrm{O} & -3.08497500 & 1.87453700 & 0.38273700 \\ \mathrm{C} & -2.02758000 & 2.29155700 & 0.95486500 \\ \mathrm{O} & -0.90636800 & 1.68733400 & 0.99219600 \\ \mathrm{C} & -0.79162600 & 1.91012700 & -3.76492600 \\ \mathrm{H} & -0.16947600 & 2.77225000 & -3.50256800 \\ \mathrm{H} & -0.18026200 & 1.23570400 & -4.37505300 \\ \mathrm{H} & -1.66094600 & 2.23697500 & -4.33857200 \\ \mathrm{C} & -2.10206700 & 3.60825300 & 1.68768200 \\ \mathrm{H} & -1.14063300 & 4.12458500 & 1.63028900 \\ \mathrm{H} & -2.89778000 & 4.23358400 & 1.27672300 \\ \mathrm{H} & -2.32295700 & 3.41069200 & 2.74393700 \\ \mathrm{C} & -2.94653700 & -1.97158900 & 3.25084300 \\ \mathrm{H} & -2.58453900 & -3.00517600 & 3.20679000 \\ \mathrm{H} & -2.45569800 & -1.48230300 & 4.09831400 \\ \mathrm{H} & -4.02835300 & -1.97246300 & 3.39654200 \\ \mathrm{C} & -1.57024400 & -3.65673600 & -2.30866100 \\ \mathrm{H} & -1.09542800 & -4.41651200 & -1.67993600 \\ \mathrm{H} & -2.53996600 & -4.01196300 & -2.66138700 \\ \mathrm{H} & -0.91297900 & -3.47842200 & -3.16819200 \\ \mathrm{H} & 2.02096000 & -2.11885700 & 0.54889500 \\ \mathrm{C} & 3.80711900 & -1.08597200 & -0.43367500 \\ \mathrm{C} & 4.54115500 & -0.78494800 & 0.72076500 \\ \mathrm{H} & 4.46749900 & -1.36489500 & -1.63287400 \\ \mathrm{H} & 5.93367200 & -0.74944700 & 0.66359200 \\ \mathrm{H} & -04454000 & -0.56082100 & 1.65752700 \\ \mathrm{H} & -1.86376000 & -1.32406600 & -1.67528200 \\ \mathrm{H} & -1.60194800 & -2.52214700 \\ \mathrm{H} & -1.01632200 & -0.53320500 \\ \mathrm{H} & -0.50785900 & 1.56287600 \\ \mathrm{H} & -1.53330700 & -2.61405500 \\ \mathrm{H} & -0.98311700 & -0.57309100 \\ \mathrm{H} & -1.29782400 & -1.66084600 \\ \mathrm{H} & -1.66719300 & -1.51724900\end{array}$




\section{3-TS2}

\begin{tabular}{|c|c|c|c|}
\hline $\mathrm{Rh}$ & -0.79305600 & -0.07752400 & 0.00454400 \\
\hline $\mathrm{Rh}$ & -3.19833700 & 0.25384800 & -0.32746800 \\
\hline $\mathrm{C}$ & 1.65877400 & -0.15689700 & 0.26938600 \\
\hline $\mathrm{C}$ & 1.51383900 & -0.96031100 & 1.46408300 \\
\hline $\mathrm{O}$ & 1.41740100 & -0.26864400 & 2.59424100 \\
\hline $\mathrm{C}$ & 1.13159200 & -0.99999200 & 3.80446100 \\
\hline $\mathrm{H}$ & 0.25766000 & -1.63495900 & 3.66841900 \\
\hline $\mathrm{H}$ & 0.93819900 & -0.23414000 & 4.55642200 \\
\hline $\mathrm{H}$ & 1.99798400 & -1.60213800 & 4.09329100 \\
\hline $\mathrm{O}$ & 1.44190700 & -2.23168100 & 1.54617400 \\
\hline $\mathrm{C}$ & 2.24950600 & 1.22723000 & 0.40499900 \\
\hline $\mathrm{C}$ & 1.87249500 & 2.26329100 & -0.46460700 \\
\hline $\mathrm{C}$ & 2.51173700 & 3.50463900 & -0.42658700 \\
\hline $\mathrm{C}$ & 3.54437800 & 3.74129700 & 0.48337100 \\
\hline $\mathrm{C}$ & 3.93944200 & 2.71784500 & 1.34834900 \\
\hline $\mathrm{C}$ & 3.30432400 & 1.47623800 & 1.30255000 \\
\hline $\mathrm{H}$ & 3.64079900 & 0.68624400 & 1.96514300 \\
\hline $\mathrm{H}$ & 4.75160700 & 2.87897500 & 2.05292800 \\
\hline $\mathrm{H}$ & 4.03944200 & 4.70861800 & 0.51451900 \\
\hline $\mathrm{H}$ & 2.19530600 & 4.28999900 & -1.10892300 \\
\hline $\mathrm{N}$ & 2.27187900 & -0.91958500 & -0.86755700 \\
\hline $\mathrm{H}$ & 1.06630400 & 2.10441400 & -1.16689900 \\
\hline $\mathrm{O}$ & -1.06569700 & -1.95460100 & -0.90219600 \\
\hline $\mathrm{C}$ & -2.24116100 & -2.33571200 & -1.26376900 \\
\hline $\mathrm{O}$ & -3.28999800 & -1.64547800 & -1.15108400 \\
\hline $\mathrm{O}$ & -2.76358100 & 1.03714200 & -2.20165800 \\
\hline $\mathrm{C}$ & -1.56270900 & 1.10295600 & -2.58447200 \\
\hline $\mathrm{O}$ & -0.54772200 & 0.74292800 & -1.88363700 \\
\hline $\mathrm{O}$ & -3.49686200 & -0.53687900 & 1.54781000 \\
\hline $\mathrm{C}$ & -2.49118200 & -0.93003400 & 2.21803800 \\
\hline $\mathrm{O}$ & -1.27588300 & -0.90054000 & 1.83228700 \\
\hline $\mathrm{O}$ & -2.96417200 & 2.12737100 & 0.50433500 \\
\hline $\mathrm{C}$ & -1.81518000 & 2.47352900 & 0.92492100 \\
\hline $\mathrm{O}$ & -0.75389600 & 1.76790700 & 0.87319900 \\
\hline $\mathrm{C}$ & -1.25951000 & 1.66528700 & -3.94752500 \\
\hline $\mathrm{H}$ & -0.72384300 & 2.61420800 & -3.82821700 \\
\hline $\mathrm{H}$ & -0.60147200 & 0.98009900 & -4.49150800 \\
\hline $\mathrm{H}$ & -2.17785600 & 1.83289800 & -4.51270300 \\
\hline $\mathrm{C}$ & -1.67192400 & 3.83128500 & 1.56546900 \\
\hline $\mathrm{H}$ & -0.75795100 & 4.31333200 & 1.20639800 \\
\hline $\mathrm{H}$ & -2.54182500 & 4.45614900 & 1.35374600 \\
\hline $\mathrm{H}$ & -1.57751000 & 3.70197000 & 2.65027800 \\
\hline $\mathrm{C}$ & -2.73644700 & -1.48098500 & 3.60097600 \\
\hline
\end{tabular}


$\mathrm{H}$

$\mathrm{H}$

$\mathrm{H}$

C

$\mathrm{H}$

$\mathrm{H}$

$\mathrm{H}$

$\mathrm{H}$

C

C

C

C

$\mathrm{H}$

C

$\mathrm{H}$

C

$\mathrm{H}$

$\mathrm{H}$

$\mathrm{H}$

$\mathrm{O}$

$\mathrm{H}$

$\mathrm{O}$

$\mathrm{H}$

$\mathrm{H}$

\section{3-INT3 \\ -INT3}

$\mathrm{C}$

C

O

C

$\mathrm{H}$

$\mathrm{H}$

$\mathrm{H}$

$\mathrm{O}$

C

C

C

C

C

C

$\mathrm{H}$

$\mathrm{H}$

$\mathrm{H}$

$\mathrm{H}$

C
$-2.33701700$

$-2.20220300$

$-3.80275200$

$-2.33807900$

$-1.94270900$

$-3.37250200$

$-1.72174600$

1.69563600

3.72521900

4.32581200

4.51827900

5.71685100

3.71345600

5.90926300

4.04721400

6.51530300

6.17408700

6.51943900

7.59823600

1.92099200

0.98527800

1.17857500

0.23722200

1.27878600
$-2.49847000$

$-0.86542500$

$-1.48742000$

$-3.71280700$

$-4.45038300$

$-3.95606400$

$-3.75593200$

$-2.42499300$

$-0.95758700$

$-1.87797900$

$-0.10974100$

$-1.93484700$

$-2.53812200$

$-0.18313500$

0.60008300

$-1.09031500$

$-2.65108800$

0.48033600

$-1.14236400$

$-0.33344300$

$-0.04984200$

$-3.25777900$

$-3.00256500$

$-2.86083800$
3.66670600

4.33277300

3.83413600

$-1.86647800$

$-1.15908900$

$-2.11377400$

$-2.77146700$

$-0.96291400$

$-0.82365300$

0.04414400

$-1.60382400$

0.14162400

0.64792300

$-1.50653900$

$-2.27072400$

$-0.63441300$

0.81898400

$-2.11389100$

$-0.56299900$

$-2.13977600$

$-2.05362300$

$-0.61873200$

$-0.83929600$

0.48808400

$-0.73140700$

$-0.49233200$

0.35378800

$-1.55761300$

$-1.55069500$

0.07127400

$-2.81113800$

$-1.39862900$

$-0.35306600$

$-3.57235700$

$-2.57425600$

$-0.69682900$

$-3.09096000$

$-3.12796500$

$-1.50801900$

$-4.54085300$

$-2.19544800$

$-1.02546900$

$-3.69931800$

$-3.22402300$

0.17329300

$-1.16085700$

$-2.82659700$

0.20924200

$-1.03232300$

0.94106700

0.19851600

$-0.21859700$

1.89714200

0.84562700

$-0.44895200$

3.26433700

0.69663000

$-1.50063000$

3.72688200

$-0.09820800$

$-2.31470900$

2.79465300

$-0.74723500$

$-2.08572000$

1.42592900

$-0.60798200$

$-2.72372500$

0.72854000

$-1.13594500$

$-3.13373500$

3.13276400

$-1.37789900$

$-1.68170100$

4.79249000

$-0.21133900$

0.19841700

3.97087900

1.21071300 


$\begin{array}{lrrc}\mathrm{N} & 0.53622300 & -0.92877500 & 0.86331500 \\ \mathrm{O} & 0.66007100 & -0.66227600 & 2.28044000 \\ \mathrm{H} & 0.59800000 & 1.56804100 & 1.47774100 \\ \mathrm{C} & 2.95053500 & -0.43357300 & 0.83854900 \\ \mathrm{C} & 1.72833000 & -0.58256400 & 0.16618600 \\ \mathrm{C} & 1.69441800 & -0.46436800 & -1.23436900 \\ \mathrm{C} & 2.86521300 & -0.19440700 & -1.94104000 \\ \mathrm{C} & 4.08459300 & -0.03902600 & -1.27438400 \\ \mathrm{C} & 4.11468700 & -0.16194700 & 0.11628600 \\ \mathrm{H} & 0.75311500 & -0.58221300 & -1.76078800 \\ \mathrm{H} & 2.81898300 & -0.10227700 & -3.02327900 \\ \mathrm{H} & 4.99350700 & 0.17426100 & -1.82995600 \\ \mathrm{H} & 5.05234700 & -0.04296500 & 0.65372700 \\ \mathrm{H} & 2.98011100 & -0.52080000 & 1.91721300 \\ \mathrm{H} & 0.58620700 & -1.54736400 & 2.68191200 \\ \mathrm{H} & -0.22136500 & -2.76355200 & 0.50111500\end{array}$

\section{3-TS3_1H2O}

$\begin{array}{lrrr}\mathrm{C} & -0.65935200 & -0.43258300 & -0.02640400 \\ \mathrm{C} & -1.75545100 & -1.36028900 & 0.10669700 \\ \mathrm{O} & -2.96925600 & -0.84495000 & 0.31130800 \\ \mathrm{C} & -4.08915600 & -1.74830400 & 0.21898800 \\ \mathrm{H} & -4.10947400 & -2.24300100 & -0.75638100 \\ \mathrm{H} & -4.97336900 & -1.12263300 & 0.34616000 \\ \mathrm{H} & -4.04293100 & -2.50337400 & 1.00822400 \\ \mathrm{O} & -1.62391900 & -2.61868700 & -0.03186100 \\ \mathrm{C} & -0.91489000 & 1.03523000 & 0.02264400 \\ \mathrm{C} & -1.79081300 & 1.63907700 & -0.89929200 \\ \mathrm{C} & -2.00918200 & 3.01577100 & -0.89297900 \\ \mathrm{C} & -1.33868000 & 3.83200100 & 0.02494700 \\ \mathrm{C} & -0.45667900 & 3.24897800 & 0.93797400 \\ \mathrm{C} & -0.24981300 & 1.86742600 & 0.94024500 \\ \mathrm{H} & 0.43101100 & 1.42768500 & 1.66211600 \\ \mathrm{H} & 0.07131800 & 3.86992200 & 1.65784100 \\ \mathrm{H} & -1.49966700 & 4.90694100 & 0.02418000 \\ \mathrm{H} & -2.69404300 & 3.45520400 & -1.61454100 \\ \mathrm{~N} & 0.52562500 & -0.95414600 & 0.59095400 \\ \mathrm{O} & 0.39637100 & -1.08111200 & 2.02867600 \\ \mathrm{H} & -2.30377500 & 1.01451500 & -1.62649800 \\ \mathrm{C} & 2.91706700 & -0.77944500 & 1.05592800 \\ \mathrm{C} & 1.81237700 & -0.52360400 & 0.22027600 \\ \mathrm{C} & 2.03986100 & 0.11451000 & -1.01623600 \\ \mathrm{C} & 3.33483400 & 0.45508300 & -1.40711700 \\ \mathrm{C} & 4.42972900 & 0.19070300 & -0.58077600\end{array}$


C

$\mathrm{H}$

$\mathrm{H}$

$\mathrm{H}$

$\mathrm{H}$

$\mathrm{H}$

$\mathrm{H}$

$\mathrm{H}$

$\mathrm{O}$

$\mathrm{H}$

$\mathrm{H}$

\section{3-TS3 $2 \mathrm{H}_{2} \mathrm{O}$}

$\mathrm{C}$

C

O

C

$\mathrm{H}$

$\mathrm{H}$

$\mathrm{H}$

$\mathrm{O}$

C

C

C

C

C

C

$\mathrm{H}$

$\mathrm{H}$

$\mathrm{H}$

$\mathrm{H}$

$\mathrm{N}$

$\mathrm{O}$

$\mathrm{H}$

C

C

C

C

C

C

$\mathrm{H}$

$\mathrm{H}$

$\mathrm{H}$

$\mathrm{H}$

4.20395700

$-0.42448600$

0.65404700

1.20712300

0.35970800

0.94725200

0.46565900

$-0.63414700$

$-1.25205500$

$-2.03479700$

$-2.70078700$

$-2.26329100$

$-2.33572300$

$-1.25743200$

$-0.24840000$

$-1.25743200$

$-1.66505200$

$-2.36568800$

$-0.88788300$

1.31882300

2.01639200

2.14070500

$-1.04470600$

$-1.85564300$

$-2.68926200$

$-1.43460200$

0.68485300

$-0.23116000$

$-0.12306000$

1.74165800

$-1.16942000$

$-0.34503400$

2.99517600

$-0.70578500$

$-0.15939300$

4.05940400

$-1.66069100$

$-0.29456800$

3.95883100

$-2.47093500$

0.43477900

4.97575100

$-1.098666100$

$-0.10507100$

4.08118700

$-2.08473100$

$-1.30254400$

1.57591800

$-2.38020400$

$-0.67535100$

0.95171100

1.23369400

$-0.06871500$

1.82479300

1.78460500

0.88917400

2.04165000

3.16031700

0.96211100

1.37522700

4.02900300

0.09125100

0.49564500

3.49871800

$-0.85644100$

2.12027900

$-0.93757400$

0.28821000

1.72089400

$-1.68537500$

$-0.02986600$

4.15990900

$-1.54168600$

1.53653400

5.10207700

0.15405200

2.72389900

3.55687300

1.71068000

$-0.53148600$

$-0.70122600$

$-0.71626900$

$-0.44968800$

$-0.82315000$

$-2.16021200$

2.33721300

1.12080700

1.57941800

$-2.93275800$

$-0.56381300$

$-1.11317700$

$-1.80613700$

$-0.27957000$

$-0.31358800$

$-2.00861600$

0.38375900

0.91502700

$-3.29691800$

0.71687300

1.33352000

0.42429200

0.54397300

$-0.21460300$

$-0.68378300$

$-4.21167400$

0.64496800

1.53986400

1.22654900

2.28604800

$-3.42180900$

0.69513400

0.87272600

$-5.06184900$

$-0.44816100$

$-1.32068400$ 
$\mathrm{H}$

$\mathrm{H}$

$\mathrm{H}$

$\mathrm{O}$

$\mathrm{H}$

$\mathrm{H}$

$\mathrm{O}$

$\mathrm{H}$

$\mathrm{H}$

\section{3-TS3}

$\mathrm{C}$

C

O

C

$\mathrm{H}$

$\mathrm{H}$

$\mathrm{H}$

$\mathrm{O}$

C

C

C

C

C

C

$\mathrm{H}$

$\mathrm{H}$

$\mathrm{H}$

$\mathrm{H}$

$\mathrm{N}$

$\mathrm{O}$

$\mathrm{H}$

C

C

C

C

C

C

$\mathrm{H}$

$\mathrm{H}$

$\mathrm{H}$

$\mathrm{H}$

$\mathrm{H}$

$\mathrm{H}$

$\begin{array}{rrr}-2.79002200 & -1.05753000 & -2.06603700 \\ -0.04457200 & -1.70566300 & -2.25702700 \\ 0.39998400 & -3.06442900 & 0.01649000 \\ 0.11077200 & -1.56614400 & 2.23331900 \\ 0.90972300 & -1.70754800 & 2.77999800 \\ 0.40050100 & -0.91270400 & 1.45012000 \\ -0.24464300 & -3.43848600 & 0.74050200 \\ 0.15775900 & -4.26815900 & 1.06705400 \\ -0.12090200 & -2.58365100 & 1.57547100\end{array}$

0.73542800

$-0.34761700$

$-0.10917200$

1.32806300

$-1.64536800$

$-0.13765900$

2.67463100

$-1.66573700$

0.04786200

$-2.95815900$

0.18153300

2.87498800

$-3.49799500$

1.04536600

4.34315900

$-2.76698500$

0.32822300

3.12586300

$-3.56231900$

$-0.71791400$

0.72094700

$-2.74548600$

$-0.24673500$

1.55433900

0.89362800

$-0.12559800$

2.56881100

1.12978100

0.82472000

3.29364100

2.32096700

0.82967900

3.32486600

$-0.10477800$

3.11346400

$-1.04575300$

2.00565200

1.91615200

$-1.05744900$

1.76457800

$-1.80050300$

0.51052900

3.88213300

$-1.78015300$

3.57624500

4.25688800

$-0.09484100$

4.07046800

2.47126800

$-0.30517900$

1.57623700

$-0.57490800$

$-0.49890100$

$-0.67524900$

$-0.59585100$

0.36568500

$-2.11061900$

2.78591800

0.86781900

0.62063500

1.56459000

$-2.68578600$

$-1.54364900$

1.26989900

$-1.04188800$

$-0.25322800$

0.99587000

2.10241700

1.44526900

2.33213100

0.66726300

1.71022100

$-0.58158300$

1.13858800

1.60964100

2.58778300

2.41265400

$-2.36160900$

2.98707300

1.02086100

$-4.56644500$

1.88012600

$-1.21187600$

$-2.76941300$

0.39635700

$-2.01273400$

$-1.05707500$

$-1.36173800$ 
$\mathrm{H}$

$\mathrm{O}$

$\mathrm{H}$

$\mathrm{H}$

$\mathrm{O}$

$\mathrm{H}$

$\mathrm{H}$

$\mathrm{O}$

$\mathrm{H}$

$\mathrm{H}$

\section{4-TS1}

$\mathrm{Rh}$

$\mathrm{Rh}$

C

C

$\mathrm{O}$

C

$\mathrm{H}$

$\mathrm{H}$

$\mathrm{H}$

$\mathrm{O}$

C

C

C

C

C

C

$\mathrm{H}$

$\mathrm{H}$

$\mathrm{H}$

$\mathrm{H}$

$\mathrm{O}$

$\mathrm{N}$

$\mathrm{H}$

$\mathrm{O}$

C

$\mathrm{O}$

$\mathrm{O}$

C

$\mathrm{O}$

$\mathrm{O}$

C

$\mathrm{O}$
$-0.71696600$

$-0.49836000$

0.00589700

0.04320300

$-1.67312200$

$-1.84714800$

$-2.34401800$

$-2.58680000$

$-3.00512700$

$-1.54719300$

$-2.87946500$

$-1.51182200$

$-2.34803100$

$-0.95730000$

$-2.89103200$

$-3.79339300$

$-2.42440500$

$-2.09587700$

$-2.84475400$

$-1.79202200$

$-0.87006000$

2.15351800

2.22207700

1.45807600

$-1.21674900$

$-1.53766200$

0.11992600

1.06084200

1.52932700

1.61932800

$-0.86975000$

$-3.21676900$

0.02108100

$-0.19595700$

1.15461300

0.22569000

$-0.83963000$

1.56027600

1.90816700

2.15609200

$-2.01242200$

$-3.07155200$

2.97432500

2.43001700

1.25462800

1.47543200

1.86051200

1.48345300

2.18736300

3.26941200

3.65710300

2.97481700

3.29638100

4.50347200

3.81182000

1.88777700

1.90219400

3.03776000

0.64939800

$-0.62419700$

$-1.62508000$

$-2.80197700$

$-2.58691400$

$-1.36745100$

$-2.80577000$

$-3.93723000$

$-3.28382400$

$-0.62851300$

1.50574900

2.55858500

3.76060300

3.94965700

2.92118500

1.70834700

0.91503900

3.05924700

4.89114600

4.55276400

$-0.44742800$

0.18135900

2.41434100

$-1.95867700$

$-2.62424000$

$-2.18459200$

0.32892200

0.53882300

0.52875100

0.15895700

$-0.55117200$

0.68375300

1.63567600

1.10505500

2.04868400

2.72416500

1.44471300

2.63081500

2.83788700

0.74681300

$-0.12107100$

$-0.13273500$

0.73270700

1.59821200

1.59430100

2.25867000

2.26494800

0.72914200

$-0.81308100$

$-1.07397000$

$-1.65786800$

$-0.79569200$

$-0.37267400$

$-0.80481900$

$-0.98074300$

$-2.47502800$

$-2.70609700$

$-1.81718900$

$-3.64815100$

$-0.71046800$

1.40247000

$-2.70932900$

$-0.77640500$

2.25443900

$-1.47069600$

$-0.55208700$

2.03625100 


$\begin{array}{lrrr}\mathrm{O} & -3.45876500 & 1.80654300 & -0.07341300 \\ \mathrm{C} & -2.47959400 & 2.44559100 & 0.41981000 \\ \mathrm{O} & -1.30944700 & 1.98318000 & 0.64028400 \\ \mathrm{C} & -0.92459200 & 0.83183000 & -4.11574900 \\ \mathrm{H} & -0.35279600 & 1.76545700 & -4.13372500 \\ \mathrm{H} & -0.25949800 & 0.02946700 & -4.45560900 \\ \mathrm{H} & -1.78285400 & 0.90437300 & -4.78574300 \\ \mathrm{C} & -2.70633400 & 3.88455100 & 0.81745800 \\ \mathrm{H} & -2.87969800 & 3.92590600 & 1.89983900 \\ \mathrm{H} & -1.81730500 & 4.48151100 & 0.59630200 \\ \mathrm{H} & -3.57937400 & 4.29477000 & 0.30539000 \\ \mathrm{C} & -3.08119700 & -1.11782400 & 3.67706500 \\ \mathrm{H} & -2.27009800 & -1.66482700 & 4.16386100 \\ \mathrm{H} & -3.24226200 & -0.18326400 & 4.22882000 \\ \mathrm{H} & -4.00520500 & -1.69998200 & 3.70240200 \\ \mathrm{C} & -1.35787500 & -4.07772800 & -1.11724700 \\ \mathrm{H} & -1.12887300 & -4.60641800 & -0.18472200 \\ \mathrm{H} & -2.22405700 & -4.54015400 & -1.59433200 \\ \mathrm{H} & -0.48169800 & -4.15996200 & -1.76822200 \\ \mathrm{H} & 1.13457600 & -0.18428900 & -1.64716700 \\ \mathrm{H} & 2.97968300 & 1.17929100 & -1.43301000 \\ \mathrm{C} & 4.23747100 & -0.38443100 & -1.15195700 \\ \mathrm{C} & 4.33830600 & -1.75892300 & -0.89958200 \\ \mathrm{C} & 5.35298400 & 0.44727800 & -0.98028200 \\ \mathrm{C} & 5.55529300 & -2.29010500 & -0.47231700 \\ \mathrm{H} & 3.46405900 & -2.38805400 & -1.01144200 \\ \mathrm{C} & 6.56892300 & -0.10089000 & -0.57162500 \\ \mathrm{H} & 5.26303400 & 1.51616500 & -1.15849400 \\ \mathrm{C} & 6.67661700 & -1.47022700 & -0.31148200 \\ \mathrm{H} & 5.62581100 & -3.35503500 & -0.26653100 \\ \mathrm{H} & 7.42909200 & 0.55018600 & -0.44074000 \\ \mathrm{H} & 7.62148900 & -1.89222100 & 0.01903200\end{array}$

\section{4-INT2}

$\mathrm{Rh}$

$\mathrm{Rh}$

C

C

$\mathrm{O}$

$\mathrm{C}$

$\mathrm{H}$

$\mathrm{H}$

$\mathrm{H}$

$\mathrm{O}$

1.01036000
3.11841800
-2.80219800
-2.41918800
-2.75630300
-2.53179800
-3.11328700
-2.87401600
-1.46987100
-1.90672400

0.01855900

$-0.36216000$

$-0.58584400$

0.60676700

0.43875400

0.29917800

$-0.69160100$

$-0.70069900$

$-1.88223800$

$-0.20244200$

$-3.01317500$

$-1.06695200$

$-2.90716600$

$-1.98734200$

$-3.88087800$

$-0.50217300$

$-3.09593300$

$-1.30298900$

$-0.54810600 \quad-1.79603300$ 


\begin{tabular}{|c|c|c|c|}
\hline $\mathrm{C}$ & -2.22272000 & 1.81503400 & -0.07687400 \\
\hline $\mathrm{C}$ & -1.87262600 & 2.68365300 & 0.97035400 \\
\hline $\mathrm{C}$ & -1.37863900 & 3.96270600 & 0.71303400 \\
\hline $\mathrm{C}$ & -1.23010600 & 4.40796800 & -0.60267200 \\
\hline $\mathrm{C}$ & -1.59010200 & 3.56160600 & -1.65262100 \\
\hline $\mathrm{C}$ & -2.08345300 & 2.27914900 & -1.39604000 \\
\hline $\mathrm{H}$ & -2.35185500 & 1.64135600 & -2.22615600 \\
\hline $\mathrm{H}$ & -1.48798300 & 3.89433200 & -2.68235600 \\
\hline $\mathrm{H}$ & -0.84447800 & 5.40350700 & -0.80642400 \\
\hline $\mathrm{H}$ & -1.11111400 & 4.61046900 & 1.54401500 \\
\hline $\mathrm{O}$ & -2.38907500 & 0.06077000 & 1.59788200 \\
\hline $\mathrm{N}$ & -4.96362500 & -0.91777600 & 2.30343900 \\
\hline $\mathrm{H}$ & -1.98509000 & 2.35067000 & 1.99567300 \\
\hline $\mathrm{O}$ & 0.39609500 & -1.92020500 & -0.07218300 \\
\hline $\mathrm{C}$ & 1.18099300 & -2.74180400 & 0.50546400 \\
\hline $\mathrm{O}$ & 2.36157300 & -2.47833500 & 0.90666200 \\
\hline $\mathrm{O}$ & 2.37011600 & -0.02716900 & 2.45348400 \\
\hline $\mathrm{C}$ & 1.15903400 & 0.33180100 & 2.54735400 \\
\hline $\mathrm{O}$ & 0.35088200 & 0.43651100 & 1.55812400 \\
\hline $\mathrm{O}$ & 3.75505100 & -1.10809200 & -1.28027700 \\
\hline $\mathrm{C}$ & 2.97313000 & -0.92621700 & -2.26982400 \\
\hline $\mathrm{O}$ & 1.78581100 & -0.46983300 & -2.19811700 \\
\hline $\mathrm{O}$ & 3.75384800 & 1.34693700 & 0.27532600 \\
\hline $\mathrm{C}$ & 2.93998900 & 2.18125900 & -0.24212700 \\
\hline $\mathrm{O}$ & 1.74243600 & 1.92638700 & -0.59322100 \\
\hline $\mathrm{C}$ & 0.61314200 & 0.69153600 & 3.90193600 \\
\hline $\mathrm{H}$ & 0.42514600 & 1.77120100 & 3.92896800 \\
\hline $\mathrm{H}$ & -0.34300800 & 0.18356400 & 4.06254700 \\
\hline $\mathrm{H}$ & 1.31774400 & 0.42432900 & 4.69103200 \\
\hline $\mathrm{C}$ & 3.44412100 & 3.57810600 & -0.49459100 \\
\hline $\mathrm{H}$ & 3.83960600 & 3.62504100 & -1.51693400 \\
\hline $\mathrm{H}$ & 2.62507900 & 4.29660400 & -0.41152400 \\
\hline $\mathrm{H}$ & 4.24834200 & 3.82921700 & 0.20044800 \\
\hline $\mathrm{C}$ & 3.50631600 & -1.23916200 & -3.64324500 \\
\hline $\mathrm{H}$ & 2.69586700 & -1.55603700 & -4.30400600 \\
\hline $\mathrm{H}$ & 3.95143300 & -0.32621800 & -4.05836800 \\
\hline $\mathrm{H}$ & 4.28008900 & -2.00834200 & -3.58999100 \\
\hline $\mathrm{C}$ & 0.67126000 & -4.14607000 & 0.69853500 \\
\hline $\mathrm{H}$ & 0.75945700 & -4.68423000 & -0.25318400 \\
\hline $\mathrm{H}$ & 1.25694300 & -4.67062400 & 1.45606300 \\
\hline $\mathrm{H}$ & -0.38495800 & -4.12436300 & 0.97938800 \\
\hline $\mathrm{H}$ & -1.41114100 & 0.14602000 & 1.64249700 \\
\hline $\mathrm{H}$ & -4.40546500 & -0.19220000 & 2.76198100 \\
\hline $\mathrm{C}$ & -5.03732200 & -0.67917400 & 1.04185700 \\
\hline
\end{tabular}




$\begin{array}{lccc}\mathrm{C} & -5.79496000 & -1.61057600 & 0.19969900 \\ \mathrm{C} & -4.39330300 & 0.54055800 & 0.36215600 \\ \mathrm{C} & -6.12602100 & -1.30320600 & -1.07222200 \\ \mathrm{H} & -6.11242900 & -2.53848200 & 0.66755500 \\ \mathrm{C} & -4.96294700 & 0.84565900 & -1.00016800 \\ \mathrm{H} & -4.55689000 & 1.40282300 & 1.02092500 \\ \mathrm{C} & -5.74045400 & -0.02594600 & -1.66523700 \\ \mathrm{H} & -6.71320700 & -1.99587100 & -1.66994800 \\ \mathrm{H} & -4.69522800 & 1.79826300 & -1.44514700 \\ \mathrm{H} & -6.11192900 & 0.21701500 & -2.65735500\end{array}$

\section{4-INT3}

$\begin{array}{lrrr}\mathrm{C} & 0.09283000 & 0.35055600 & -0.59530900 \\ \mathrm{C} & -0.43854800 & 1.31134400 & 0.48726600 \\ \mathrm{O} & -0.24292800 & 2.58974700 & 0.14194100 \\ \mathrm{C} & -0.68981200 & 3.57968100 & 1.09636000 \\ \mathrm{H} & -1.76520500 & 3.47956400 & 1.26400700 \\ \mathrm{H} & -0.45957400 & 4.54459100 & 0.64479200 \\ \mathrm{H} & -0.15578900 & 3.45978400 & 2.04262300 \\ \mathrm{O} & -0.93297400 & 0.95102400 & 1.53520100 \\ \mathrm{C} & 1.53260000 & -0.06262100 & -0.24569000 \\ \mathrm{C} & 2.40453200 & -0.39256800 & -1.29206300 \\ \mathrm{C} & 3.71118500 & -0.80489300 & -1.02317700 \\ \mathrm{C} & 4.16437700 & -0.89007400 & 0.29556700 \\ \mathrm{C} & 3.29969700 & -0.56340800 & 1.34305800 \\ \mathrm{C} & 1.99084900 & -0.15605300 & 1.07654900 \\ \mathrm{H} & 1.32732200 & 0.07754300 & 1.90244300 \\ \mathrm{H} & 3.64065200 & -0.62641400 & 2.37298700 \\ \mathrm{H} & 5.18238200 & -1.20712100 & 0.50548400 \\ \mathrm{H} & 4.37416300 & -1.05757900 & -1.84647000 \\ \mathrm{O} & 0.06591200 & 0.97206400 & -1.88165300 \\ \mathrm{~N} & -2.77674600 & 0.57182300 & -1.52149900 \\ \mathrm{H} & 2.05601700 & -0.31903600 & -2.31659300 \\ \mathrm{H} & 0.51225100 & 1.83498600 & -1.80432300 \\ \mathrm{H} & -1.98816600 & 1.00836200 & -2.00771700 \\ \mathrm{C} & -2.36051700 & -0.41507100 & -0.80370000 \\ \mathrm{C} & -3.34837800 & -1.15851700 & -0.01957000 \\ \mathrm{C} & -0.88537600 & -0.87321000 & -0.71758900 \\ \mathrm{C} & -3.00287600 & -2.17118200 & 0.80450400 \\ \mathrm{H} & -4.37969600 & -0.83113800 & -0.12046900 \\ \mathrm{C} & -0.62947500 & -1.96786900 & 0.28783000 \\ \mathrm{H} & -0.62956600 & -1.29817900 & -1.70148400 \\ \mathrm{C} & -1.61483800 & -2.58372100 & 0.96090700 \\ \mathrm{H} & -3.76605600 & -2.69043100 & 1.37906600\end{array}$




\section{4-TS3}

C

C

$\mathrm{O}$

C

$\mathrm{H}$

$\mathrm{H}$

$\mathrm{H}$

$\mathrm{O}$

C

C

C

C

C

C

$\mathrm{H}$

$\mathrm{H}$

$\mathrm{H}$

$\mathrm{H}$

$\mathrm{O}$

$\mathrm{N}$

$\mathrm{H}$

$\mathrm{H}$

$\mathrm{H}$

C

C

C

C

$\mathrm{H}$

C

$\mathrm{H}$

C

$\mathrm{H}$

$\mathrm{H}$

$\mathrm{H}$

$$
\begin{array}{r}
-0.25123300 \\
0.61904700 \\
1.54264600 \\
2.50741500 \\
3.06110700 \\
3.18000200 \\
2.01528700 \\
0.54804100
\end{array}
$$

$-1.70473000$

$-2.64240700$

$-3.98908400$

$-4.42561000$

$-3.50054900$

$-2.15046700$

$-1.44434200$

$-3.82681300$

$-5.47453700$

$-4.69762800$

$-0.09490700$

1.86491200

$-2.30081600$

0.86235500

2.77336600

1.85175600

3.00131100

0.51217600

2.85778400

3.97634800

0.45651300

$-0.19133000$

1.57533000

3.73620900

$-0.51430700$

1.52095700
$-0.67685000$

$-0.88563200$

$-1.83660400$

$-2.00513800$

$-1.07552800$

$-2.79314400$

$-2.30199800$

$-0.24772700$

$-0.28789800$

$-0.67414200$

$-0.33288100$

0.39885000

0.78474500

0.45004400

0.75794800

1.35275300

0.66421400

$-0.64361500$

$-1.61651600$

$-0.61530900$

$-1.24913900$

$-1.30901700$

$-1.07817900$

0.38402200

0.89957400

0.86902600

1.91598100

0.47416500

2.01677900

0.91569400

2.49524600

2.30576900

2.45916800

3.32449000
$-0.29916500$

0.95682400

0.74200500

1.79773100

1.96022800

1.45641200

2.72821700

1.99088100

$-0.05867700$

$-1.02942000$

$-0.89964200$

0.20782400

1.18066900

1.05055100

1.80983800

2.04829600

0.31196500

$-1.66335700$

$-1.22627000$

$-2.26874900$

$-1.88247300$

$-1.90214600$

$-2.33200700$

$-1.39928800$

$-0.71118900$

$-1.01307800$

0.19228100

$-0.93454600$

$-0.11503300$

$-1.84299400$

0.48947800

0.70062500

0.08273700

1.18832600

\section{4-INT4}

C

0.46444800

$-1.36179100$

$-0.65525000$

1.58599400

$-1.41179700$

0.40372300

$\mathrm{O}$

2.71912300

$-0.88807300$

$-0.07791500$

C

3.84791900

$-0.89522700$

0.82549400 
$\mathrm{H}$

$\mathrm{H}$

$\mathrm{H}$

$\mathrm{O}$

C

C

C

C

C

C

$\mathrm{H}$

$\mathrm{H}$

$\mathrm{H}$

$\mathrm{H}$

$\mathrm{O}$

$\mathrm{N}$

$\mathrm{H}$

$\mathrm{H}$

$\mathrm{H}$

C

C

C

C

$\mathrm{H}$

C

$\mathrm{H}$

C

$\mathrm{H}$

$\mathrm{H}$

$\mathrm{H}$

$\begin{array}{rcc}3.60118300 & -0.35908400 & 1.74597700 \\ 4.65031400 & -0.38834100 & 0.28968300 \\ 4.13391900 & -1.92302900 & 1.06376100 \\ 1.47314900 & -1.91664500 & 1.50224900 \\ -0.94484300 & -1.25103700 & -0.20927500 \\ -1.94502600 & -1.42365000 & -1.18563600 \\ -3.28699000 & -1.28803200 & -0.85055800 \\ -3.64952200 & -0.96762500 & 0.46412400 \\ -2.66407700 & -0.78468900 & 1.43651400 \\ -1.31594500 & -0.92650000 & 1.10736000 \\ -0.55956600 & -0.77192900 & 1.86542800 \\ -2.94293400 & -0.52495800 & 2.45371000 \\ -4.69845800 & -0.85803600 & 0.72630300 \\ -4.05254900 & -1.42780100 & -1.60863800 \\ 0.78811800 & -1.45638500 & -1.83111300 \\ 1.68324100 & 1.48569300 & -1.87953900 \\ -1.64808100 & -1.66269000 & -2.20169200 \\ 1.22820800 & 1.08983800 & -2.69664100 \\ 2.40873100 & 0.84869400 & -1.56341900 \\ 0.78454500 & 1.83155800 & -0.86515700 \\ 1.21375900 & 1.92586100 & 0.47377200 \\ -0.56012100 & 2.12852100 & -1.16073600 \\ 0.32339900 & 2.30717100 & 1.47802300 \\ 2.24940700 & 1.70335700 & 0.71701400 \\ -1.44074400 & 2.50824900 & -0.15030100 \\ -0.90866300 & 2.04303900 & -2.18747100 \\ -1.01012200 & 2.59995100 & 1.17791700 \\ 0.67672300 & 2.36827200 & 2.50460500 \\ -2.47745500 & 2.71826800 & -0.40148000 \\ -1.70263400 & 2.88760400 & 1.96377300\end{array}$

\section{5-TS1}

$\mathrm{Rh}$

$\mathrm{Rh}$

C

C

$\mathrm{O}$

C

$\mathrm{H}$

$\mathrm{H}$

$\mathrm{H}$

$\mathrm{O}$

C

C

$-0.69846500$

0.01339000

0.11606800

$-2.55589300$

$-1.51009300$

$-0.42220000$

0.91797600

1.36611100

0.53231100

1.51912200

0.99966600

1.84233300

2.56170800

0.15152200

1.74162200

3.07926500

$-0.36349100$

2.98427200

3.38346500

0.45325000

3.64407600

3.94019300

$-0.97148200$

2.70704900

2.32127100

$-0.97561700$

3.48110800

1.05819100

1.38028300

2.90383400

0.71438200

2.79983500

0.21544900

0.25822300

3.15072700

$-1.07317600$ 


\begin{tabular}{|c|c|c|c|}
\hline $\mathrm{C}$ & 0.05305000 & 4.48329500 & -1.41560200 \\
\hline $\mathrm{C}$ & 0.27341400 & 5.49494600 & -0.47361700 \\
\hline $\mathrm{C}$ & 0.71849700 & 5.16067600 & 0.80715000 \\
\hline $\mathrm{C}$ & 0.95328800 & 3.82760700 & 1.14791200 \\
\hline $\mathrm{H}$ & 1.29890300 & 3.58460600 & 2.14544800 \\
\hline $\mathrm{H}$ & 0.89457900 & 5.93947300 & 1.54442800 \\
\hline $\mathrm{H}$ & 0.09989600 & 6.53488300 & -0.73752600 \\
\hline $\mathrm{H}$ & -0.29108100 & 4.73458900 & -2.41547800 \\
\hline $\mathrm{O}$ & 2.21548300 & 1.05937000 & -0.66414600 \\
\hline $\mathrm{N}$ & 2.53526200 & -0.32854000 & -1.14973800 \\
\hline $\mathrm{H}$ & 0.07530400 & 2.36266900 & -1.79380100 \\
\hline $\mathrm{O}$ & 0.49779400 & -1.67688000 & 0.33148000 \\
\hline $\mathrm{C}$ & -0.00811300 & -2.84101900 & 0.12893800 \\
\hline $\mathrm{O}$ & -1.21144700 & -3.08052100 & -0.16794500 \\
\hline $\mathrm{O}$ & -1.98607300 & -1.36226900 & -2.41221000 \\
\hline $\mathrm{C}$ & -1.00667700 & -0.61818500 & -2.72072200 \\
\hline $\mathrm{O}$ & -0.29670600 & 0.06808800 & -1.91034600 \\
\hline $\mathrm{O}$ & -2.99407800 & -1.55596500 & 1.59752900 \\
\hline $\mathrm{C}$ & -2.27305200 & -0.88607700 & 2.40079600 \\
\hline $\mathrm{O}$ & -1.26408500 & -0.16848700 & 2.09412200 \\
\hline $\mathrm{O}$ & -3.74749200 & 0.16434200 & -0.64266400 \\
\hline $\mathrm{C}$ & -3.24527200 & 1.31223900 & -0.43515200 \\
\hline $\mathrm{O}$ & -2.03776000 & 1.56067900 & -0.10478000 \\
\hline $\mathrm{C}$ & -0.62710200 & -0.50585700 & -4.17765200 \\
\hline $\mathrm{H}$ & -0.82196200 & 0.51717100 & -4.51980000 \\
\hline $\mathrm{H}$ & 0.44537200 & -0.69410900 & -4.29101000 \\
\hline $\mathrm{H}$ & -1.19924700 & -1.20837900 & -4.78660300 \\
\hline $\mathrm{C}$ & -4.15571800 & 2.51056700 & -0.55775000 \\
\hline $\mathrm{H}$ & -4.50501700 & 2.79006100 & 0.44391200 \\
\hline $\mathrm{H}$ & -3.60627900 & 3.35982400 & -0.97273800 \\
\hline $\mathrm{H}$ & -5.02292800 & 2.27502800 & -1.17861200 \\
\hline $\mathrm{C}$ & -2.65532000 & -0.90426500 & 3.86196200 \\
\hline $\mathrm{H}$ & -1.76007800 & -0.88248100 & 4.48893300 \\
\hline $\mathrm{H}$ & -3.24258500 & -0.00374500 & 4.08050700 \\
\hline $\mathrm{H}$ & -3.26177800 & -1.78305700 & 4.09202100 \\
\hline $\mathrm{C}$ & 0.94526100 & -4.00337700 & 0.25830800 \\
\hline $\mathrm{H}$ & 1.47490500 & -3.94211200 & 1.21432500 \\
\hline $\mathrm{H}$ & 0.41187300 & -4.95292800 & 0.18689100 \\
\hline $\mathrm{H}$ & 1.69209700 & -3.94442700 & -0.54178700 \\
\hline $\mathrm{H}$ & 3.02879500 & 1.36295700 & -0.19329100 \\
\hline $\mathrm{H}$ & 1.89099700 & -0.90706200 & -0.60010100 \\
\hline $\mathrm{C}$ & 3.88606800 & -0.65467200 & -0.86299900 \\
\hline $\mathrm{C}$ & 4.91664200 & 0.11095400 & -1.43004300 \\
\hline $\mathrm{C}$ & 4.19008300 & -1.78042200 & -0.08271000 \\
\hline
\end{tabular}


C

$\mathrm{H}$

C

$\mathrm{H}$

C

$\mathrm{H}$

$\mathrm{H}$

$\mathrm{H}$

\section{5-INT2}

$\mathrm{Rh}$

$\mathrm{Rh}$

C

C

O

C

$\mathrm{H}$

$\mathrm{H}$

$\mathrm{H}$

O

C

C

C

C

C

C

$\mathrm{H}$

$\mathrm{H}$

$\mathrm{H}$

$\mathrm{H}$

$\mathrm{O}$

$\mathrm{N}$

$\mathrm{H}$

$\mathrm{O}$

C

O

O

C

O

$\mathrm{O}$

C

O

O

C

h

C

.

C

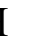

,

C

C

C

.

H

H

.

H

C

C

C

O

C

O

C
6.24615700

4.67102100

5.52160400

3.38627800

6.55229100

7.04315900

5.75478700

7.58863500

$-0.24054700$

0.96546300

$-2.14742300$

$-2.35151900$

$-1.37336200$

0.35895000

$-3.02634200$

$-1.65311100$

$-1.19534700$

$-2.05504300$

0.10870700

0.37049700

$-0.43506500$

$-1.62603000$

0.70345200

$-0.26852000$

$\begin{array}{rcc}-1.16051500 & 0.35787200 & 0.29720500 \\ -2.72619900 & -0.80238300 & -1.09409100 \\ 2.00060900 & 0.93175800 & 1.25664800 \\ 1.17934400 & 1.17517600 & 2.46916800 \\ 0.87398200 & 0.00436700 & 3.06942900 \\ -0.03130700 & 0.08305000 & 4.19059100 \\ 0.38721300 & 0.72316100 & 4.97160000 \\ -0.13180300 & -0.94141500 & 4.55011300 \\ -0.99976400 & 0.46657800 & 3.86198900 \\ 0.82358300 & 2.26881100 & 2.88429800 \\ 2.71991400 & 1.93696600 & 0.53743300 \\ 3.26404400 & 1.58967000 & -0.73147300 \\ 3.96897400 & 2.53315000 & -1.47066100 \\ 4.16719100 & 3.82225200 & -0.96700500 \\ 3.63915900 & 4.17422300 & 0.28566000 \\ 2.92094500 & 3.25366100 & 1.03336300 \\ 2.51793200 & 3.53056000 & 1.99858300 \\ 3.79557900 & 5.17557700 & 0.67693900 \\ 4.72828600 & 4.55292300 & -1.54312200 \\ 4.36715900 & 2.26031800 & -2.44404000 \\ 2.13266500 & -0.30896100 & 0.81450400 \\ 2.54417500 & -1.72392300 & -1.67943800 \\ 3.07131500 & 0.60386300 & -1.13337200 \\ -0.26513000 & -1.49559700 & 0.66716500 \\ -0.77436200 & -2.55556000 & 0.15696300 \\ -1.80190500 & -2.57678800 & -0.58559600 \\ -1.39634800 & -0.56809300 & -2.65690200 \\ -0.32536500 & 0.09709100 & -2.46154000 \\ 0.02227000 & 0.61769200 & -1.35105100 \\ -3.94689900 & -0.98285500 & 0.56234900 \\ -3.54063200 & -0.54202500 & 1.68587000 \\ -2.42713300 & 0.04592700 & 1.89229100 \\ -3.56913900 & 1.02244800 & -1.52744700 \\ -3.10515500 & 2.07101200 & -0.97184600\end{array}$




$\begin{array}{lrrr}\mathrm{O} & -2.13379500 & 2.10123600 & -0.14594300 \\ \mathrm{C} & 0.62632800 & 0.26028500 & -3.61374000 \\ \mathrm{H} & 1.00522100 & 1.28620100 & -3.64018800 \\ \mathrm{H} & 1.47583800 & -0.40976400 & -3.43022200 \\ \mathrm{H} & 0.14283800 & 0.00682800 & -4.55929400 \\ \mathrm{C} & -3.74758100 & 3.38202100 & -1.34049700 \\ \mathrm{H} & -4.83657900 & 3.28750800 & -1.29502400 \\ \mathrm{H} & -3.40876600 & 4.18125500 & -0.67884600 \\ \mathrm{H} & -3.47565400 & 3.62856900 & -2.37373500 \\ \mathrm{C} & -4.44450000 & -0.71254300 & 2.87911000 \\ \mathrm{H} & -3.88035800 & -1.16024800 & 3.70336500 \\ \mathrm{H} & -4.78810600 & 0.27464000 & 3.20907900 \\ \mathrm{H} & -5.30681500 & -1.33455300 & 2.63274500 \\ \mathrm{C} & -0.08451800 & -3.86426300 & 0.43330000 \\ \mathrm{H} & 0.45727100 & -3.82436400 & 1.38065900 \\ \mathrm{H} & -0.81154500 & -4.67964800 & 0.44247500 \\ \mathrm{H} & 0.63346400 & -4.05436600 & -0.37417500 \\ \mathrm{H} & 1.40190000 & -0.90031200 & 1.11981200 \\ \mathrm{H} & 1.73023100 & -2.07701100 & -1.17075500 \\ \mathrm{C} & 3.64344100 & -1.92610900 & -0.96090200 \\ \mathrm{C} & 4.90035500 & -1.41358800 & -1.45252100 \\ \mathrm{C} & 3.68012600 & -2.58025200 & 0.32687100 \\ \mathrm{C} & 6.07153400 & -1.56939700 & -0.73526400 \\ \mathrm{H} & 4.89390200 & -0.91056900 & -2.41589500 \\ \mathrm{C} & 4.86762400 & -2.73173500 & 1.02526800 \\ \mathrm{H} & 2.75306800 & -2.98613900 & 0.72351000 \\ \mathrm{C} & 6.07195000 & -2.22977300 & 0.50890100 \\ \mathrm{H} & 7.00337500 & -1.17499800 & -1.13379600 \\ \mathrm{H} & 4.86382300 & -3.24035400 & 1.98672700 \\ \mathrm{H} & 6.99799900 & -2.34970400 & 1.06386100\end{array}$

\section{5-INT3}

C

C

$\mathrm{O}$

$\mathrm{C}$

$\mathrm{H}$

$\mathrm{H}$

$\mathrm{H}$

$\mathrm{O}$

C

C

C

C

$\begin{array}{rr}0.47838000 & 0.35965700 \\ 1.26785600 & -0.07690100 \\ 2.58910700 & 0.07589900 \\ 3.45094700 & -0.23552100 \\ 3.31373300 & -1.27642800 \\ 4.46656000 & -0.07291900 \\ 3.23115000 & 0.42700100 \\ 0.78147600 & -0.43949100 \\ -1.00583600 & 0.06456100 \\ -1.86938700 & 1.04431600 \\ -3.21890000 & 0.74997500 \\ -3.71333500 & -0.52584600\end{array}$

0.47838000

0.35965700

$-1.97689200$

$-1.76675200$

$-2.88561400$

$-3.18995600$

$-2.52439400$

$-3.72693500$

$-3.02283200$

$-0.91342300$

$-1.41504500$

$-1.62464900$

$-1.34343600$

$-3.71333500$ 


$\begin{array}{lrrr}\mathrm{C} & -0.85416100 & -2.84907400 & -1.50942000 \\ \mathrm{C} & -0.64192200 & -1.50154800 & -1.21562000 \\ \mathrm{H} & -0.25650300 & -0.82958200 & -1.97585300 \\ \mathrm{H} & -0.63324600 & -3.22367100 & -2.50548800 \\ \mathrm{H} & -1.50501500 & -4.76384000 & -0.75301600 \\ \mathrm{H} & -2.00873800 & -3.88287600 & 1.52003000 \\ \mathrm{O} & -0.61060100 & 0.63032900 & 1.78173900 \\ \mathrm{~N} & 0.40735100 & 1.03975700 & -0.36200300 \\ \mathrm{H} & -1.63461400 & -1.48699200 & 2.03473300 \\ \mathrm{H} & -0.45631300 & 1.57719200 & 1.95291700 \\ \mathrm{H} & 0.28325000 & 2.01367500 & -0.61137300 \\ \mathrm{C} & 1.74754700 & 0.68171000 & -0.14066200 \\ \mathrm{C} & 2.14339400 & -0.38118200 & 0.69068400 \\ \mathrm{C} & 2.74179700 & 1.41644900 & -0.81638300 \\ \mathrm{C} & 3.49712300 & -0.69965600 & 0.82151300 \\ \mathrm{H} & 1.40427000 & -0.94482900 & 1.24484500 \\ \mathrm{C} & 4.08879900 & 1.09455300 & -0.66875000 \\ \mathrm{H} & 2.44473400 & 2.24026900 & -1.46229100 \\ \mathrm{C} & 4.48020900 & 0.02832900 & 0.14803500 \\ \mathrm{H} & 3.78027700 & -1.52564300 & 1.46949800 \\ \mathrm{H} & 4.83577500 & 1.67897400 & -1.20042500 \\ \mathrm{H} & 5.53056600 & -0.22555600 & 0.25979600\end{array}$

\section{5-TS3}

$\mathrm{C}$

C

$\mathrm{O}$

C

$\mathrm{H}$

$\mathrm{H}$

$\mathrm{H}$

$\mathrm{O}$

C

C

C

C

C

C

$\mathrm{H}$

$\mathrm{H}$

$\mathrm{H}$

$\mathrm{H}$

$\mathrm{O}$

$\mathrm{N}$
0.79627200

2.29072400

2.65989600

4.08056500

4.49534300

4.18971900

4.58266000

3.04874400

0.20614300

0.54922800

$-0.06267300$

$-0.99574900$

$-1.31931100$

$-0.72589700$

$-0.95276900$

$-2.03196700$

$-1.46563900$

0.19632300

1.46534200

0.08967000
0.05864200

$-0.01814900$

$-1.21533800$

$-1.41611900$

$-0.68961500$

$-2.42937100$

$-1.31864500$

0.91537100

1.39654200

2.46830800

3.71045200

3.89362100

2.83103100

1.58512900

0.75877100

2.97563500

4.86349500

4.53498500

$-0.50992900$

$-1.03426700$
$-0.13640100$

$-0.37376000$

$-0.84682200$

$-0.99999800$

$-1.70364100$

$-1.38741000$

$-0.03301000$

$-0.20182800$

0.06941800

$-0.76740800$

$-0.59175700$

0.43150900

1.28345100

1.10232500

1.76583200

2.09043100

0.57081900

$-1.24986300$

1.96942500

$-0.33193700$ 
$\mathrm{H}$

$\mathrm{H}$

$\mathrm{H}$

C

C

C

C

$\mathrm{H}$

C

$\mathrm{H}$

C

$\mathrm{H}$

$\mathrm{H}$

$\mathrm{H}$

$\mathrm{O}$

$\mathrm{H}$

$\mathrm{H}$

4a

C

C

$\mathrm{O}$

C

$\mathrm{H}$

$\mathrm{H}$

$\mathrm{H}$

$\mathrm{O}$

C

C

C

C

C

C

$\mathrm{H}$

$\mathrm{H}$

$\mathrm{H}$

$\mathrm{H}$

$\mathrm{N}$

$\mathrm{H}$

C

C

C

C

C
1.28052400

2.38056200

0.60804700

$-1.32730900$

$-2.11257700$

$-1.91796000$

$-3.49296500$

$-1.64367500$

$-3.30085900$

$-1.28884800$

$-4.09296500$

$-4.10008700$

$-3.75847500$

$-5.16934400$

1.21418600

2.06375900

1.43283200

0.54082400

0.04301800

$-0.66468400$

$-1.28396600$

$-1.99675600$

$-1.799866100$

$-0.52682400$

0.26123100

2.00625900

2.52134500

3.89476100

4.77948000

4.27885200

2.90171300

2.53095300

4.95947700

5.85208000

4.27896700

$-0.26428200$

1.83002700

$-2.44886300$

$-1.66188100$

$-2.29234400$

$-3.68569900$

$-4.46463800$
2.32753900

$-0.24442500$

$-1.94223500$

$-1.14769100$

$-0.31365000$

$-2.18513900$

$-0.50245800$

0.46815700

$-2.36243500$

$-2.83042000$

$-1.52160800$

0.14593100

$-3.16066600$

$-1.66380800$

$-2.76986200$

$-3.06725700$

$-1.70899900$

$-1.55593900$

2.16096800

0.03801100

$-0.36415000$

$-1.17141300$

0.37068900

$-1.21432500$

$-1.75879900$

0.31925500

0.97638900

$-0.46746500$

$-1.84000700$

0.89716000

$-0.50546400$

1.13909800

0.77375900

1.66552400

$\begin{array}{cc}-0.08550100 & 0.04074600 \\ 1.35252800 & 0.14352900 \\ 1.69532400 & -0.93509600 \\ 3.00134600 & -0.89356800 \\ 3.04903100 & -0.06567700 \\ 3.10738700 & -1.84789000 \\ 3.78130100 & -0.77707100 \\ 2.07522200 & 1.09535800 \\ -0.29616000 & 0.03205500 \\ -1.60613000 & 0.05590300 \\ -1.82499600 & 0.02859400 \\ -0.74090500 & -0.02545600 \\ 0.56222500 & -0.05240700 \\ 0.78642000 & -0.02261600 \\ 1.80592900 & -0.03564200 \\ 1.40795700 & -0.09429100 \\ -0.91377100 & -0.04489800 \\ -2.84107200 & 0.05099400 \\ -1.08356500 & -0.02064800 \\ -2.44133800 & 0.09578300 \\ -1.61166200 & -0.89096800 \\ -0.94721200 & 0.06515800 \\ -0.24687400 & 1.10969700 \\ -0.18755400 & 1.17031700 \\ -0.81776800 & 0.19660000\end{array}$




$\begin{array}{lrrr}\mathrm{C} & -3.83874300 & -1.52891000 & -0.83365200 \\ \mathrm{H} & -1.68953700 & 0.22819800 & 1.87787800 \\ \mathrm{H} & -4.16145000 & 0.35169300 & 1.98532600 \\ \mathrm{H} & -5.54881400 & -0.76706600 & 0.24562500 \\ \mathrm{H} & -4.43643200 & -2.03012800 & -1.59058300 \\ \mathrm{H} & -1.95492900 & -2.17061200 & -1.68071400\end{array}$




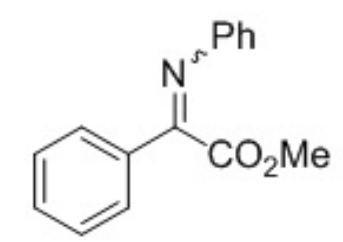

3a, $400 \mathrm{MHz}, \mathrm{CDCl}_{3}$

mixture of two geometric isomers (94:6)

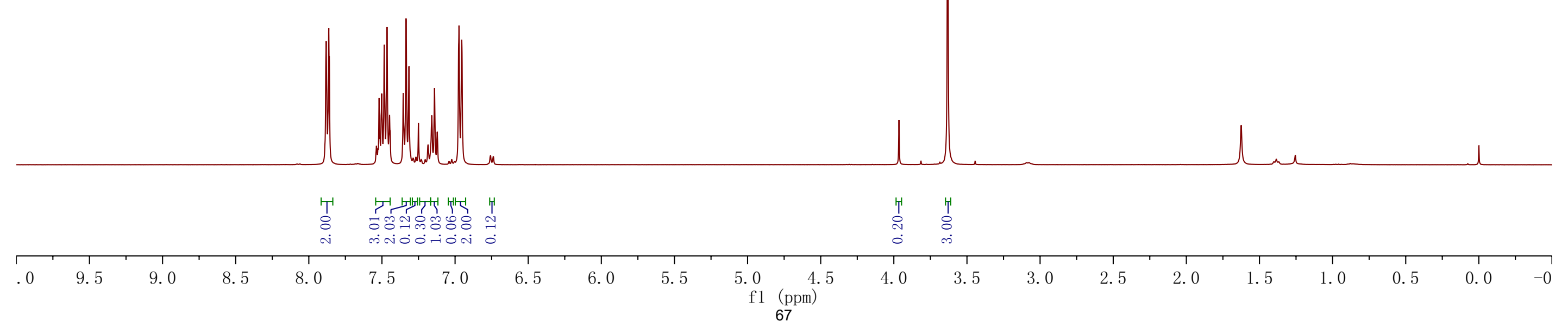


3a, $100 \mathrm{MHz}, \mathrm{CDCl}_{3}$

mixture of two geometric isomers (94:6)

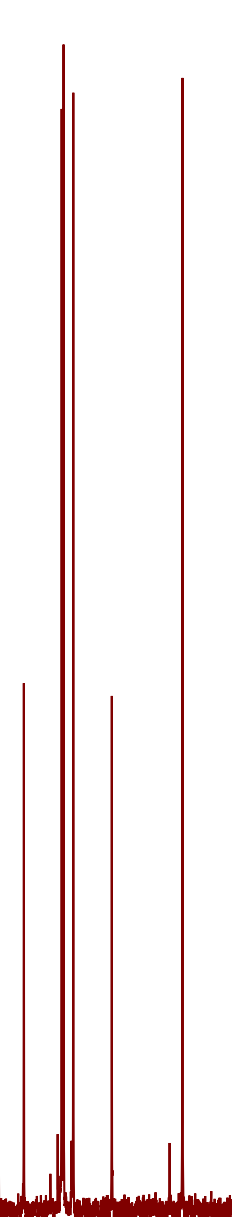

$\begin{array}{rrrrr}1 & 1 & 1 & 1 & 1 \\ 200 & 190 & 180 & 170 & 160\end{array}$

140

120 


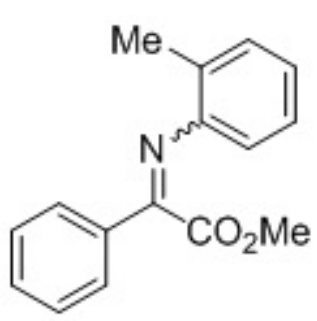

3b, $400 \mathrm{MHz}, \mathrm{CDCl}_{3}$

mixture of two geometric isomers (95:5)

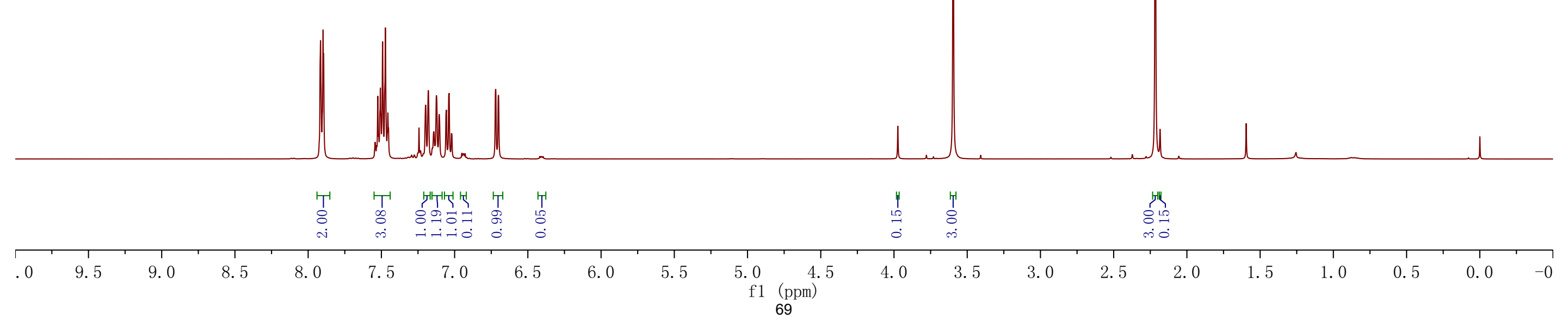




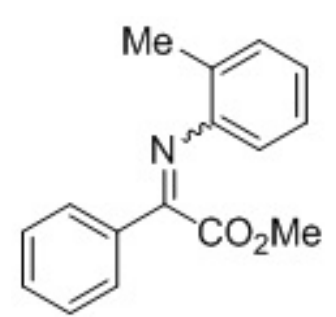

3b, $100 \mathrm{MHz}, \mathrm{CDCl}_{3}$

mixture of two geometric isomers (95:5)

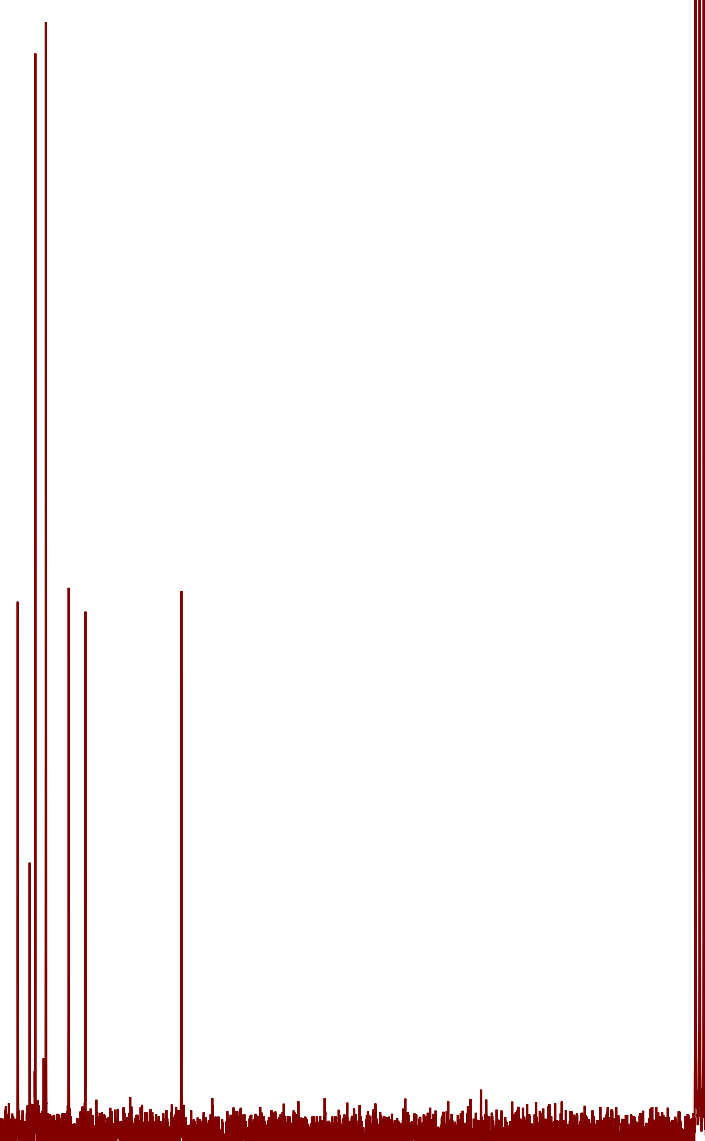

$$
\text { || }
$$

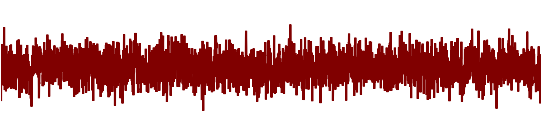




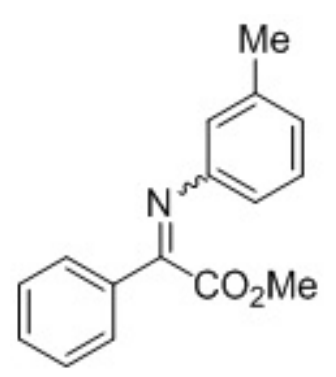

3c, $300 \mathrm{MHz}, \mathrm{CDCl}_{3}$

mixture of two geometric isomers (95:5)

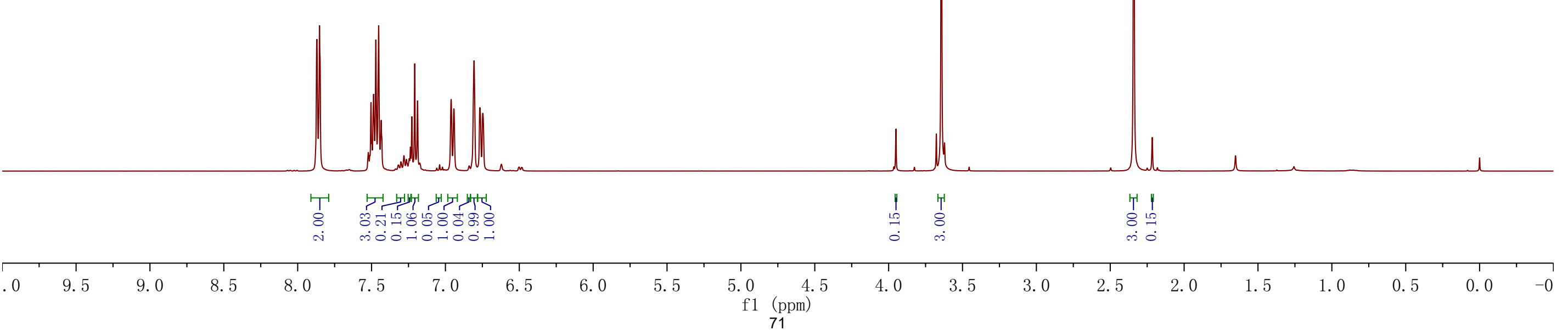




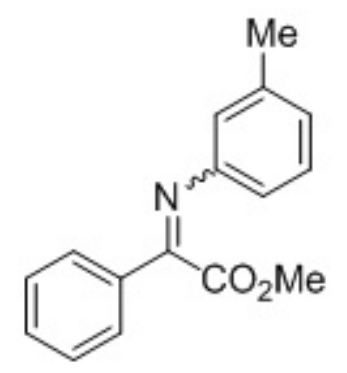

3c, $75 \mathrm{MHz}, \mathrm{CDCl}_{3}$

mixture of two geometric isomers (95:5)

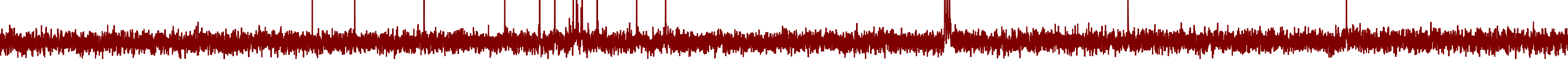

\begin{tabular}{|c|c|c|c|c|c|c|c|c|c|c|c|c|c|c|c|c|c|c|c|c|c|c|}
\hline 10 & 200 & 190 & 180 & 170 & 160 & 150 & 140 & 130 & 120 & 110 & 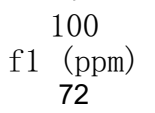 & 90 & 80 & 70 & 60 & 50 & 40 & 30 & 20 & 10 & 0 & -1 \\
\hline
\end{tabular}




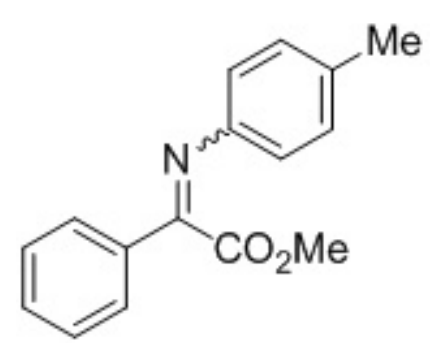

3d, $300 \mathrm{MHz}, \mathrm{CDCl}_{3}$

mixture of two geometric isomers (94:6)

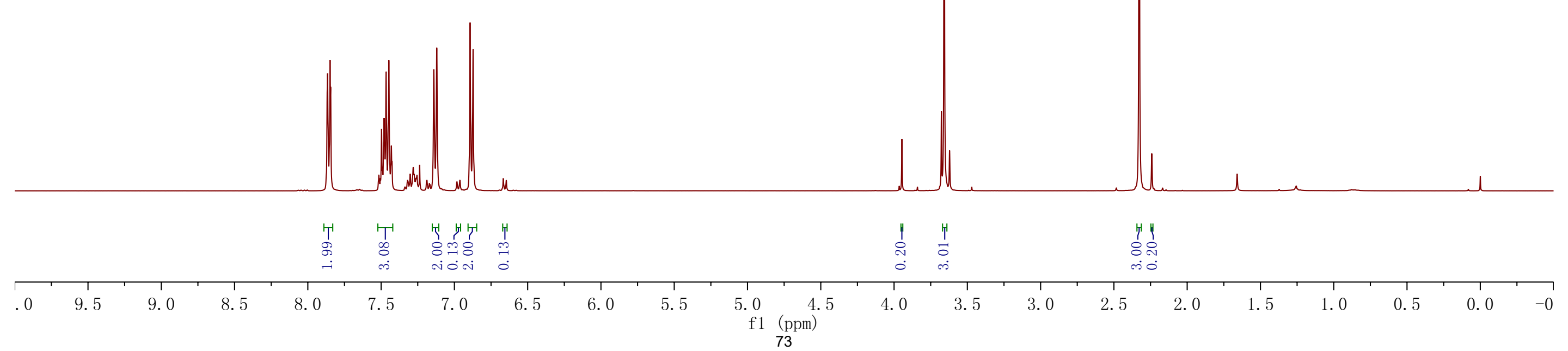




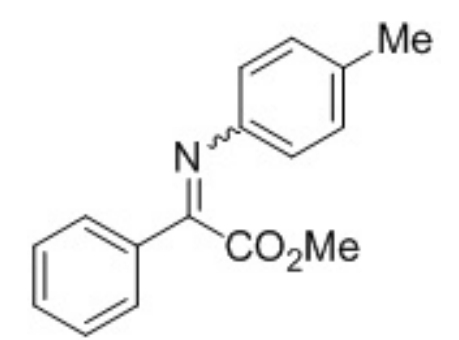

\section{3d, $75 \mathrm{MHz}, \mathrm{CDCl}_{3}$}

mixture of two geometric isomers (94:6)
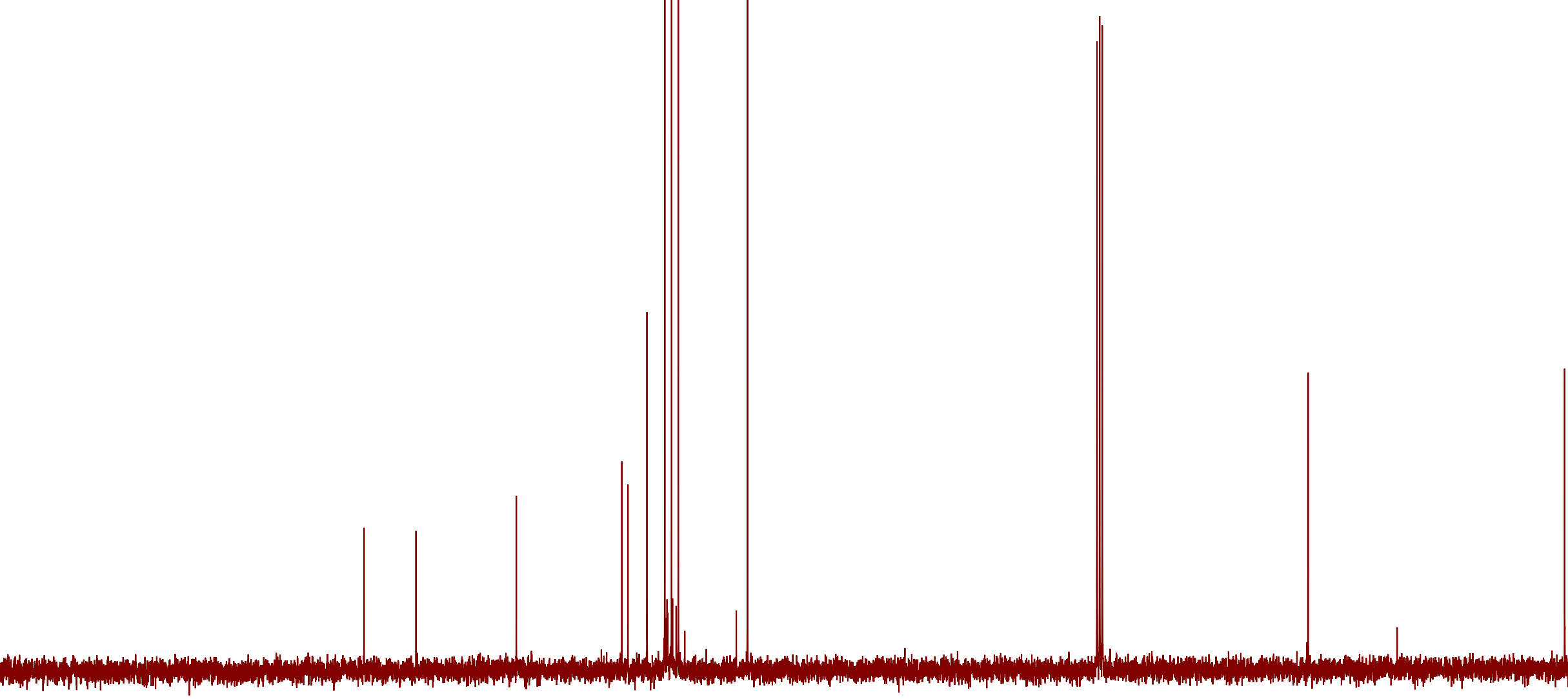

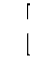

$200 \quad 190$

$180 \quad 170$

$160 \quad 150$

140

130

120

110

100

90

80 


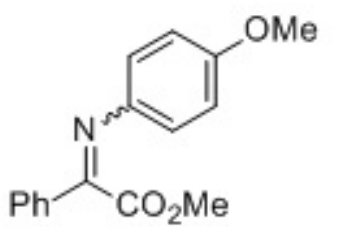

3e, $400 \mathrm{MHz}, \mathrm{CDCl}_{3}$

mixture of two geometric isomers (92:8)

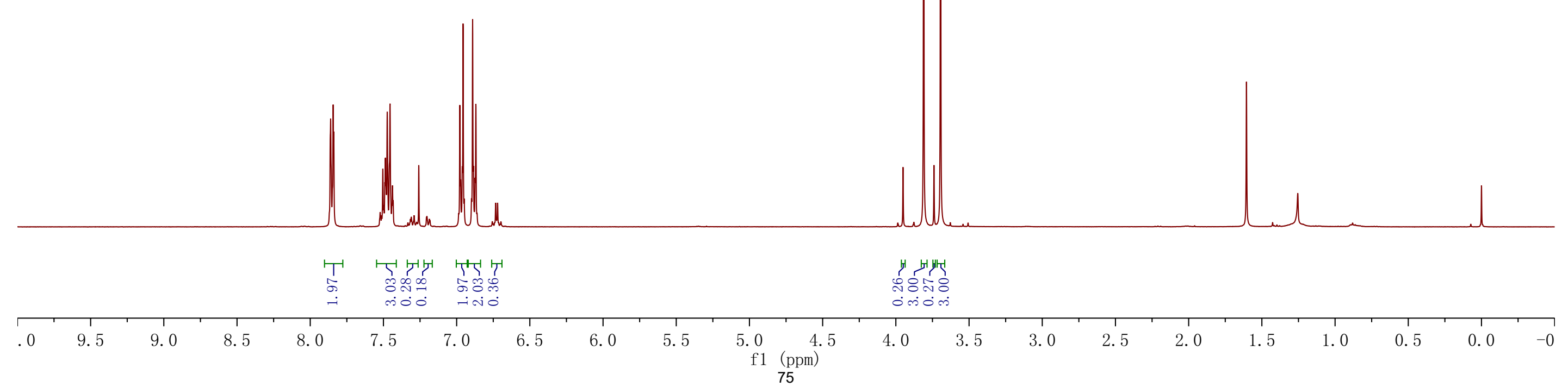


3e, $100 \mathrm{MHz}, \mathrm{CDCl}_{3}$

mixture of two geometric isomers (92:8)

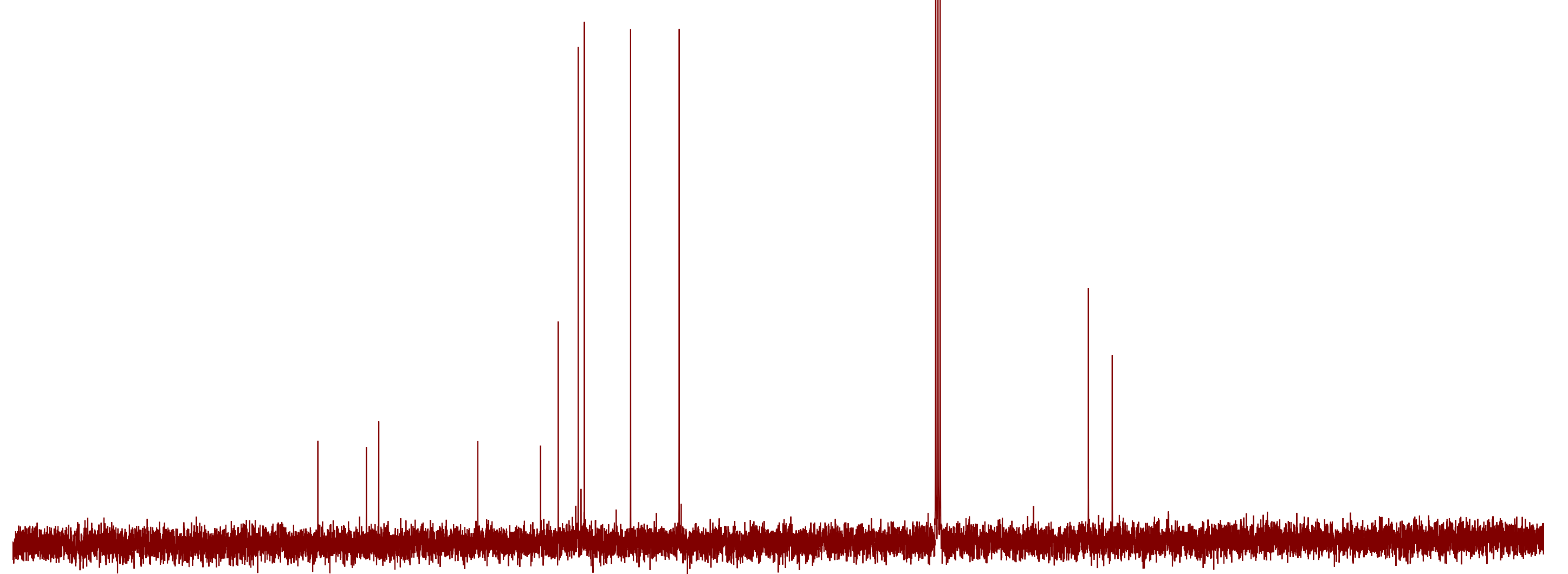




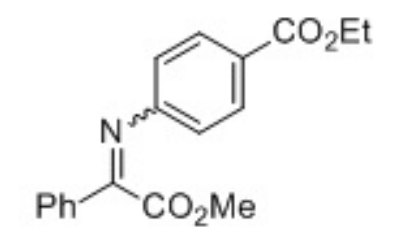

3f, $400 \mathrm{MHz}, \mathrm{CDCl}_{3}$

mixture of two geometric isomers (95:5)

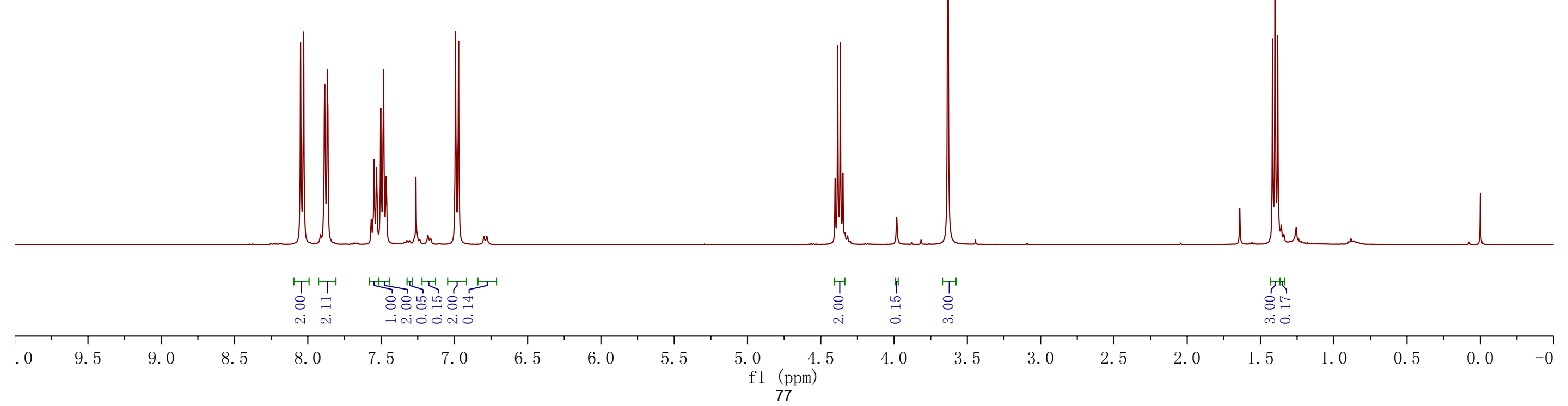



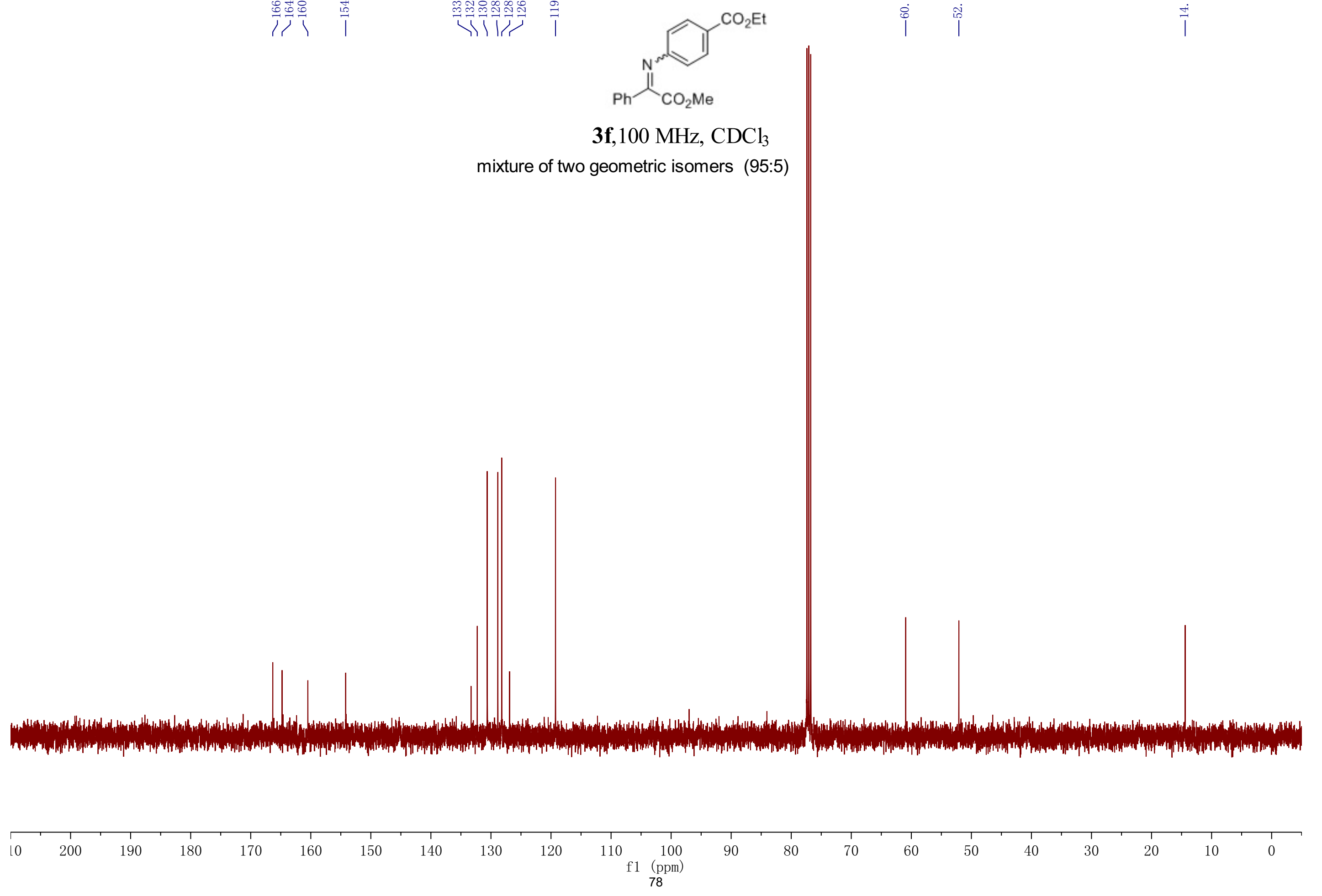


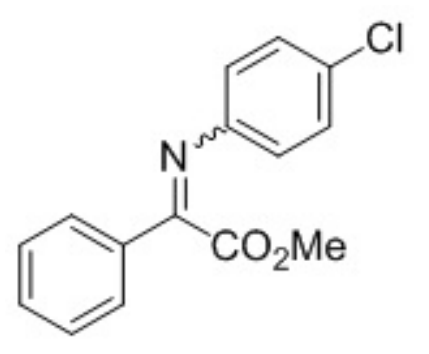

3g, $300 \mathrm{MHz}, \mathrm{CDCl}_{3}$

mixture of two geometric isomers (95:5)

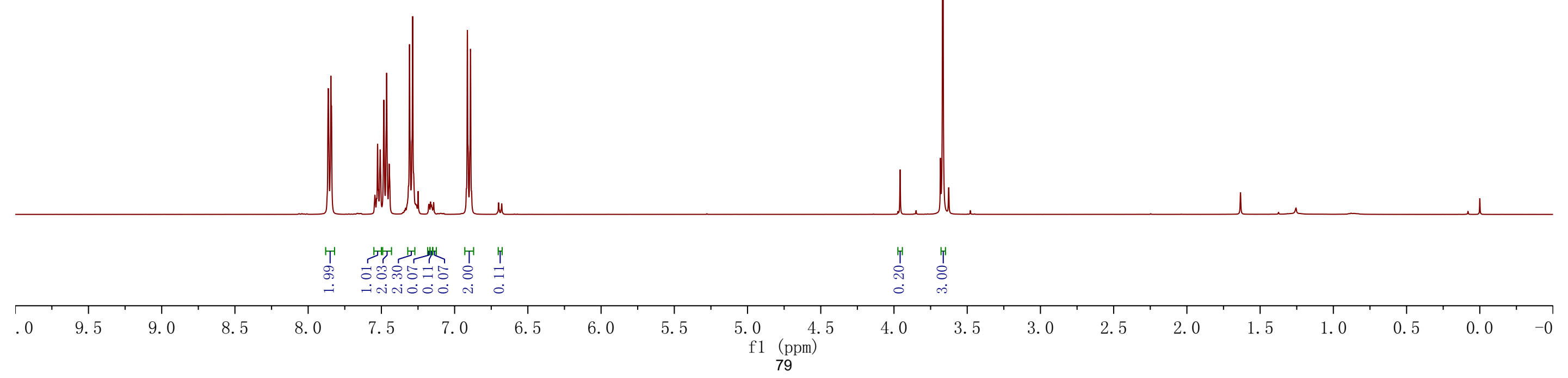




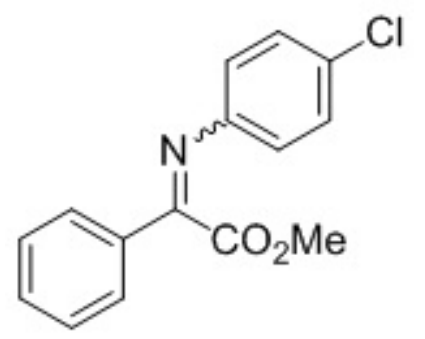

3g, $75 \mathrm{MHz}, \mathrm{CDCl}_{3}$

mixture of two geometric isomers $(95: 5)$

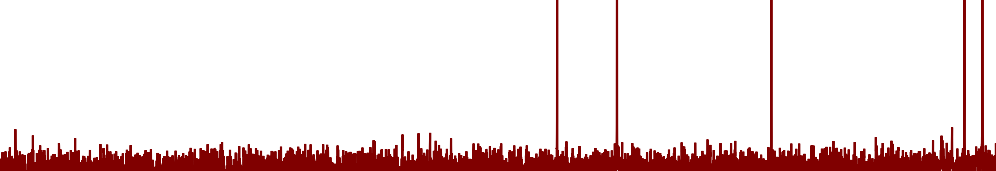

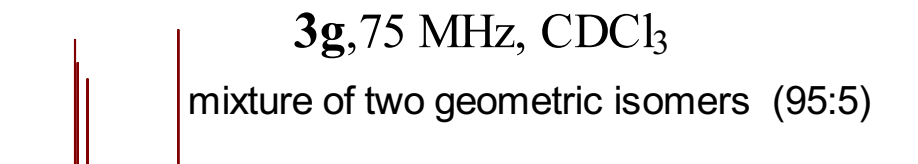

10

$0 \quad 200$

$\begin{array}{rr}180 & 170\end{array}$

$160 \quad 150$

130

120

110

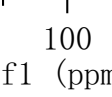

90

80

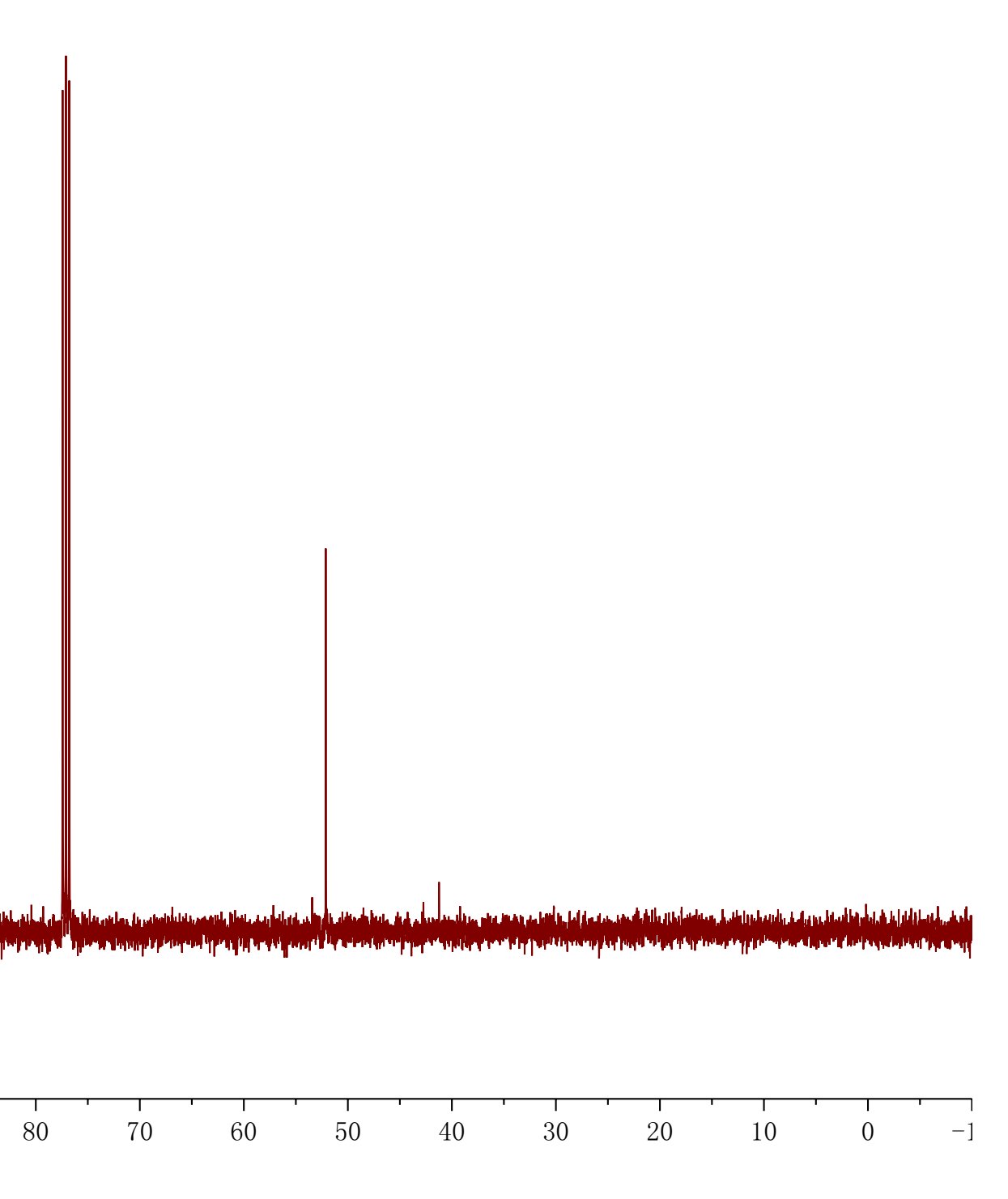




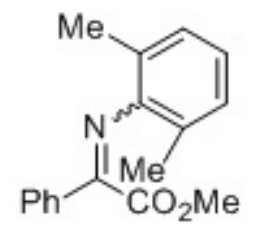

3h, $400 \mathrm{MHz}, \mathrm{CDCl}_{3}$

mixture of two geometric isomers (94:6)

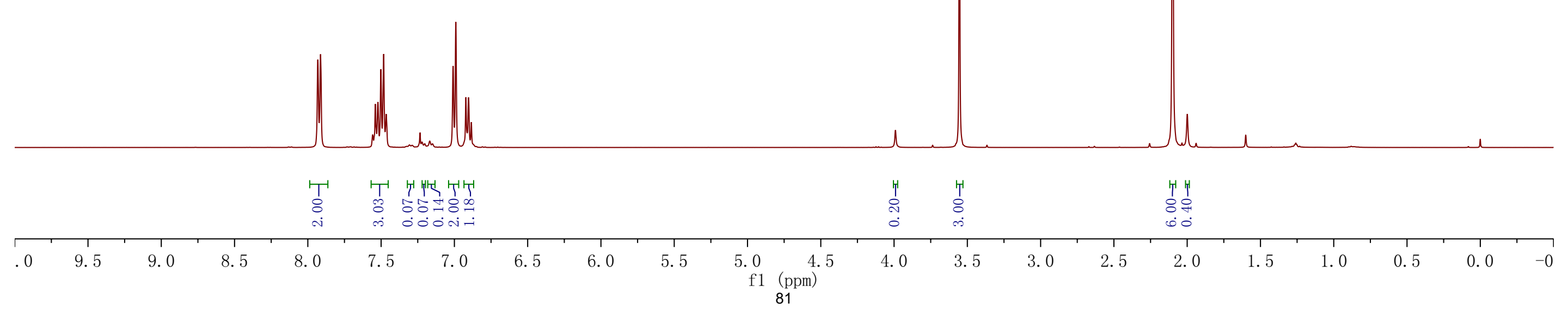




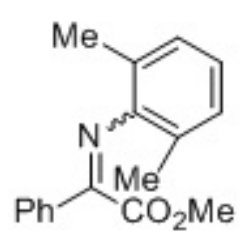

3h, $100 \mathrm{MHz}, \mathrm{CDCl}_{3}$

mixture of two geometric isomers (94:6)

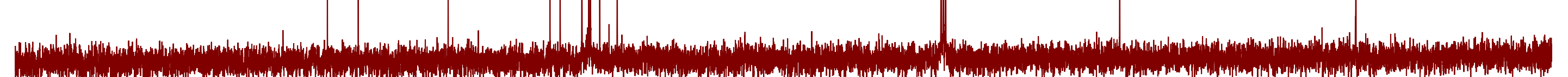

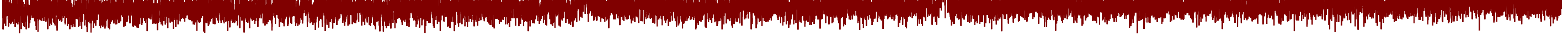

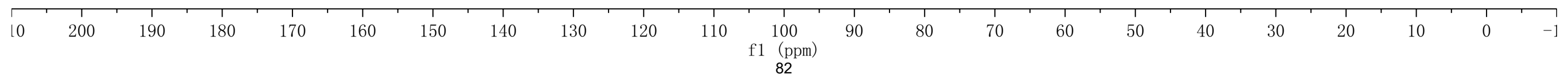




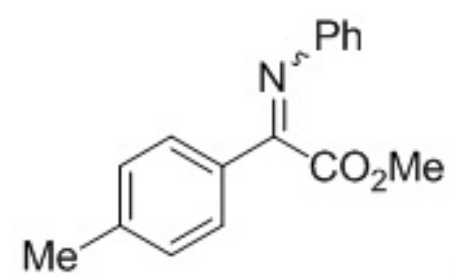

3i,300 MHz, $\mathrm{CDCl}_{3}$

mixture of two geometric isomers (95:5)

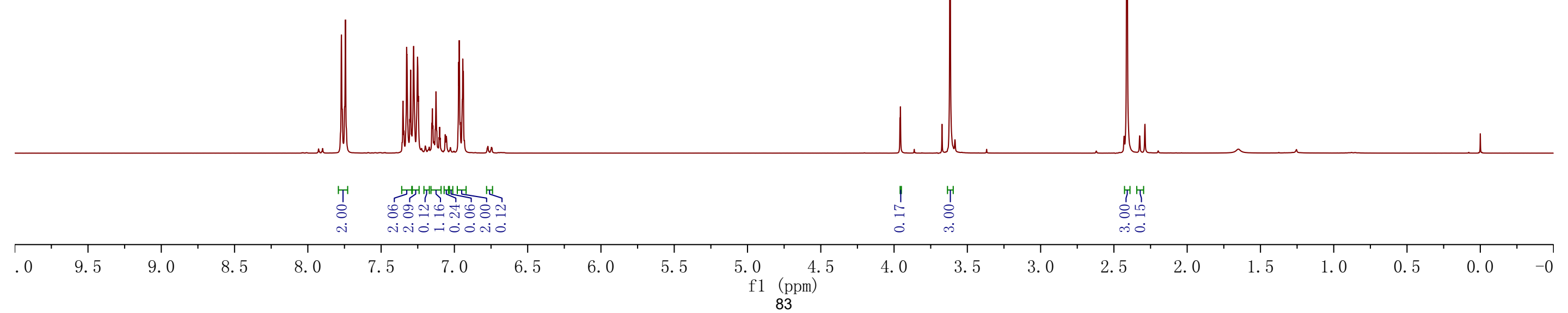




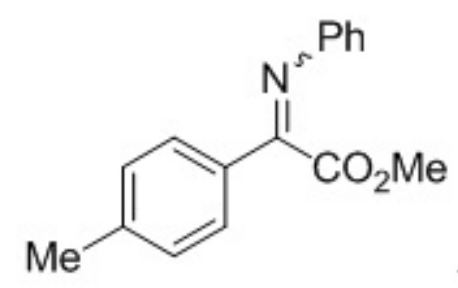

mixture of two geometric isomers (95:5)

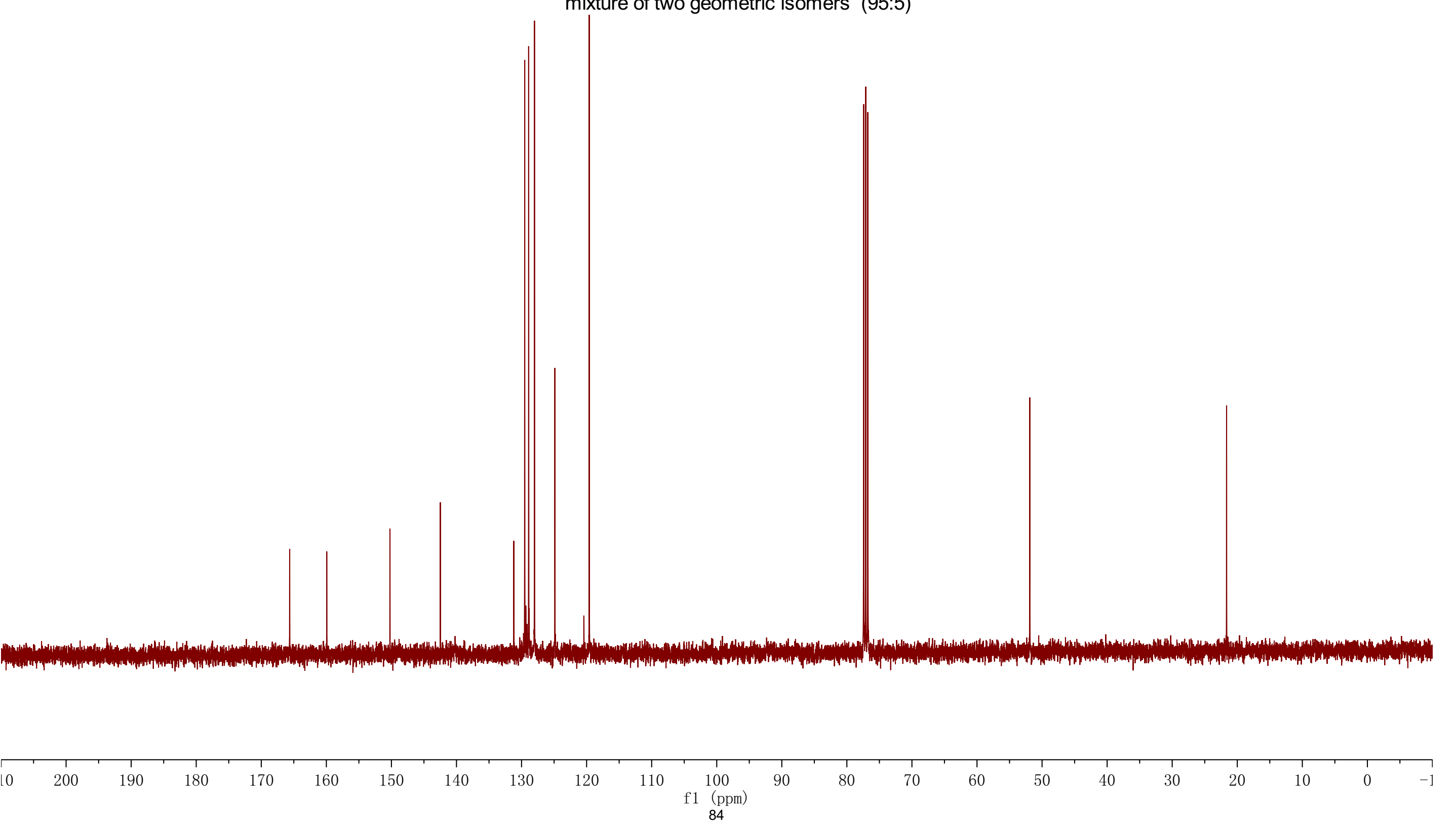




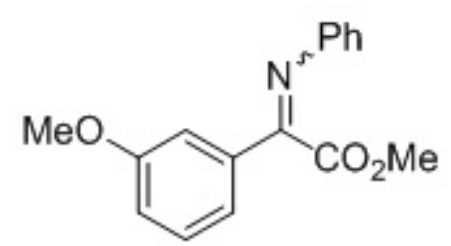

3j, $400 \mathrm{MHz}, \mathrm{CDCl}_{3}$

mixture of two geometric isomers (94:6)

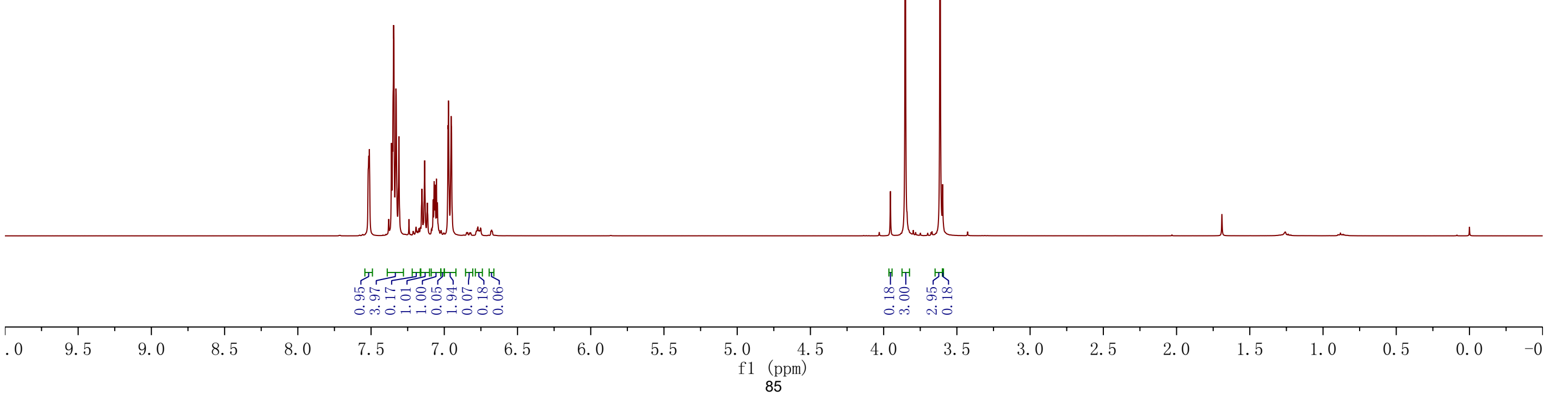




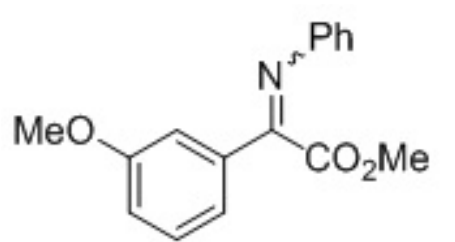

3j, $100 \mathrm{MHz}, \mathrm{CDCl}_{3}$

mixture of two geometric isomers (94:6)

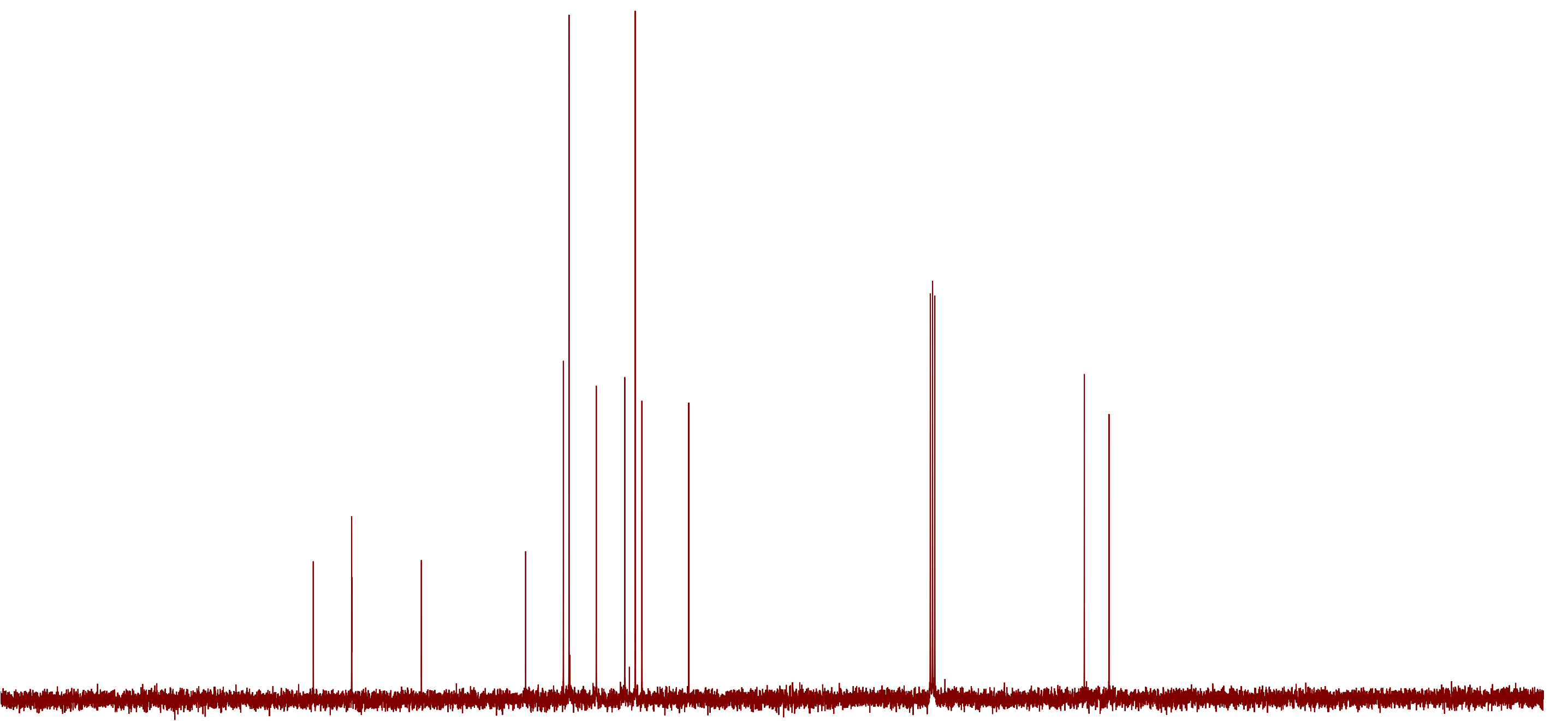

\begin{tabular}{|c|c|c|c|c|c|c|c|c|c|c|c|c|c|c|c|c|c|c|c|c|c|c|}
\hline & 1 & 1 & 1 & 1 & 1 & 1 & $T$ & 1 & 1 & $T$ & $T$ & $T$ & $T$ & $T$ & $T$ & $T$ & $T$ & $T$ & $T$ & $T$ & $T$ & \\
\hline 10 & 200 & 190 & 180 & 170 & 160 & 150 & 140 & 130 & 120 & 110 & $\begin{array}{c}100 \\
\mathrm{f} 1 \quad(\mathrm{ppm})\end{array}$ & 90 & 80 & 70 & 60 & 50 & 40 & 30 & 20 & 10 & 0 & -1 \\
\hline
\end{tabular}




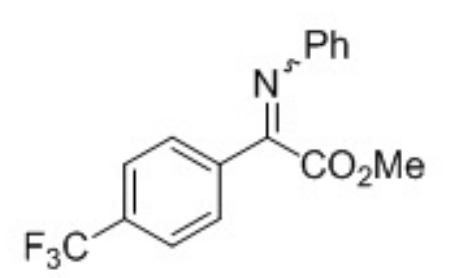

3k,300 MHz, $\mathrm{CDCl}_{3}$

mixture of two geometric isomers (93:7)

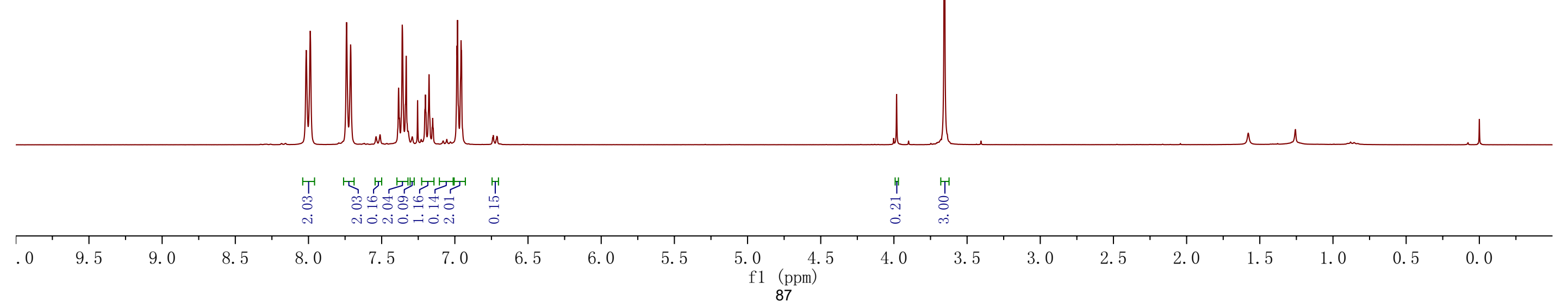




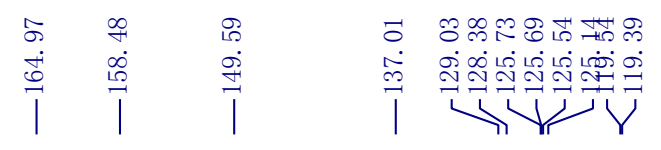

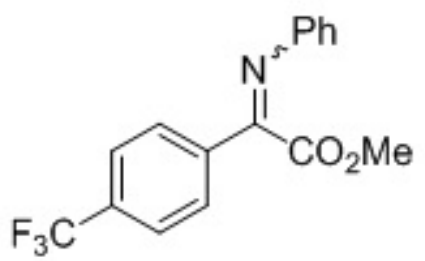

3k, $100 \mathrm{MHz}, \mathrm{CDCl}_{3}$ mixture of two geometric isomers (93:7)

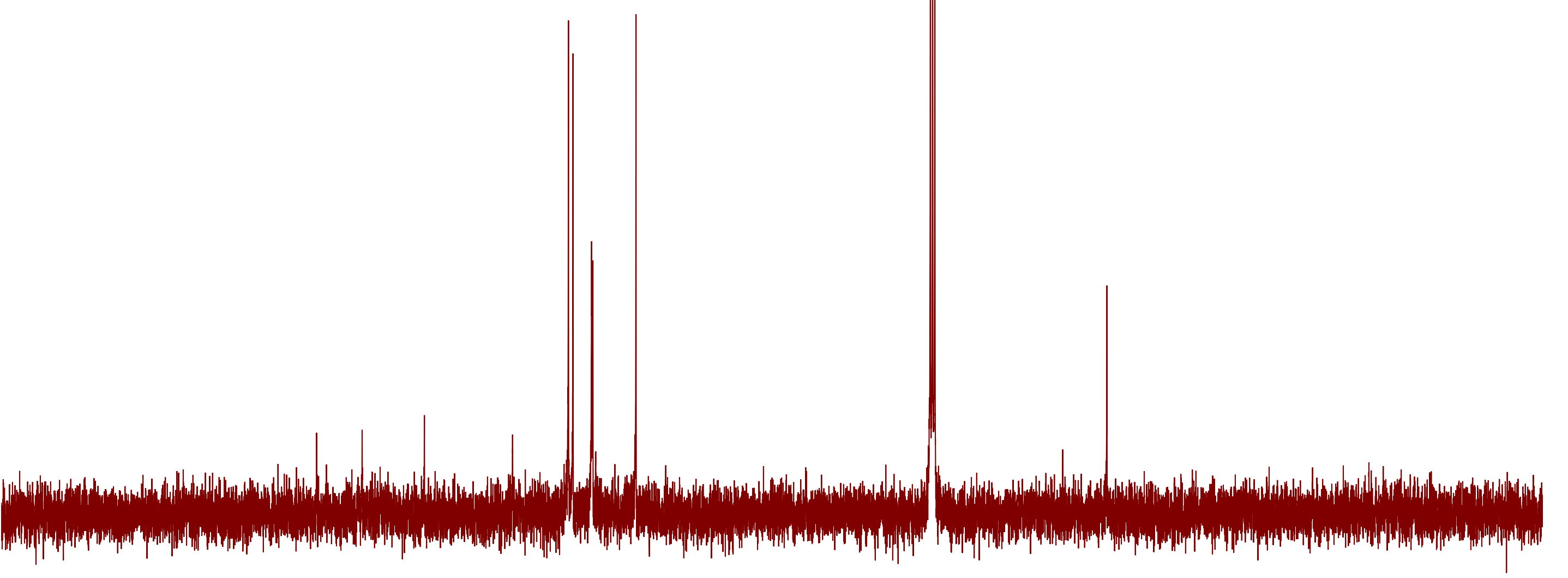




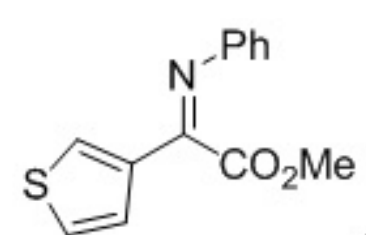

31,400 MHz, $\mathrm{CDCl}_{3}$

mixture of two geometric isomers (89:11)

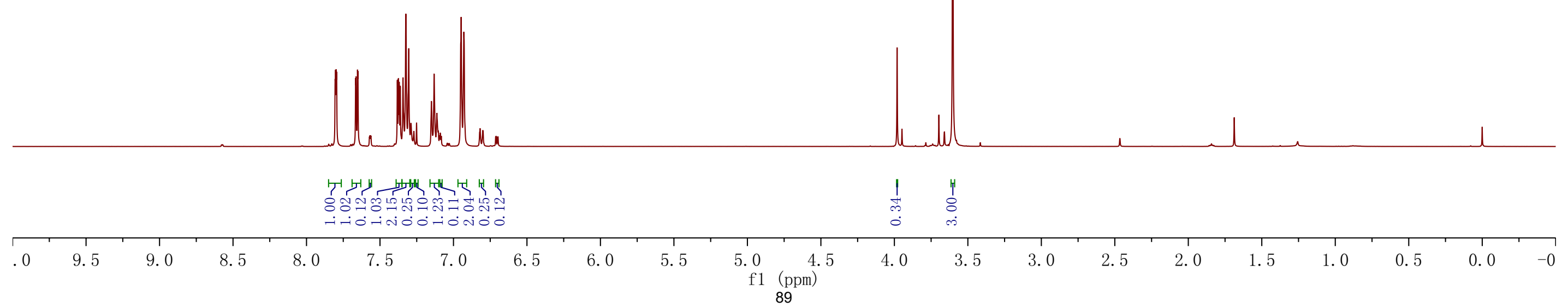




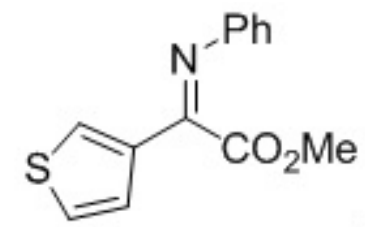

31,400 MHz, $\mathrm{CDCl}_{3}$

mixture of two geometric isomers (89:11)

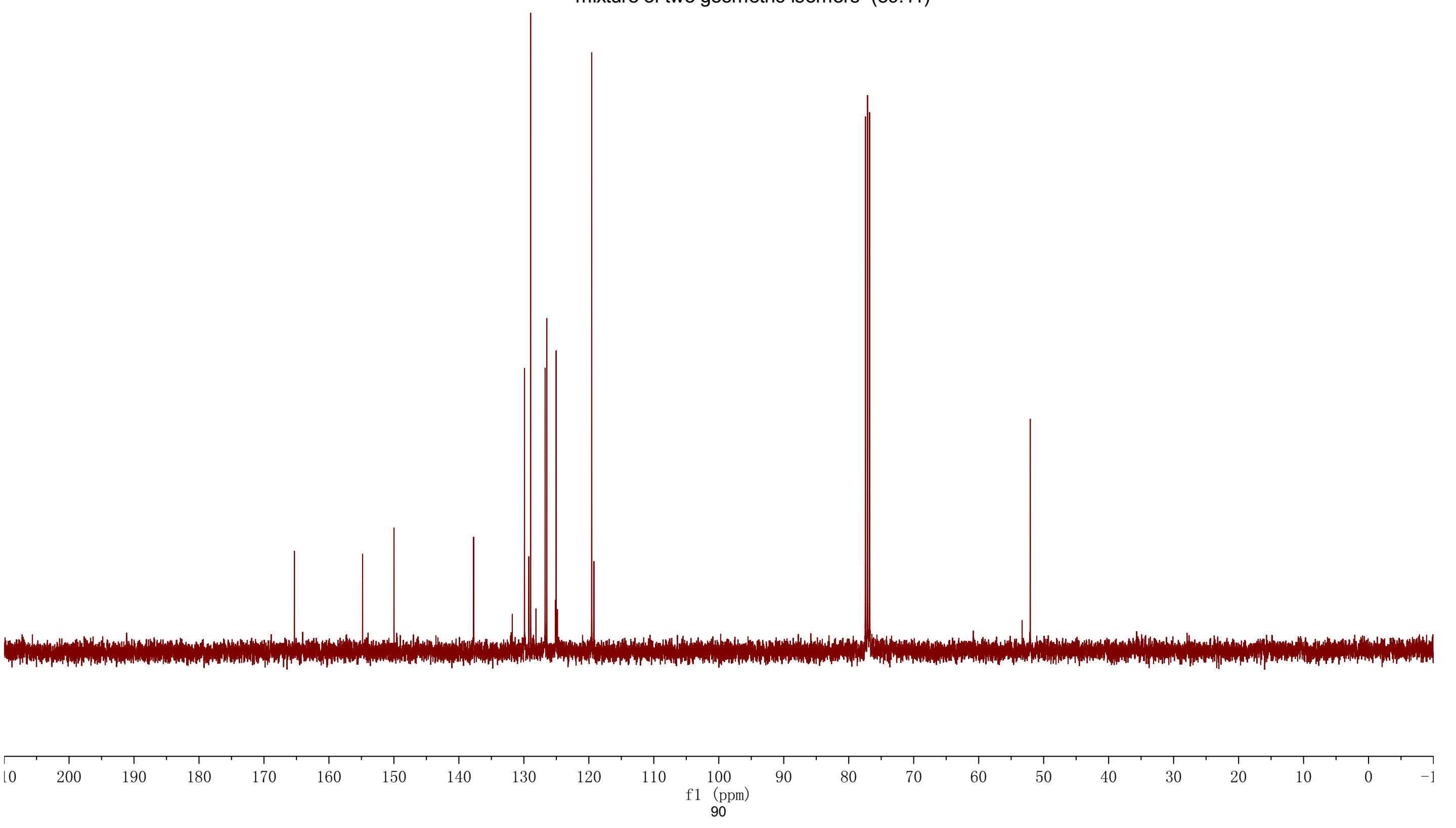




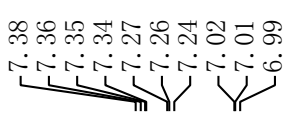

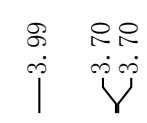

$\stackrel{8}{+}$

$\stackrel{8}{8}$<smiles>COC(=O)C(=Nc1ccccc1)C(=O)OC</smiles>

$$
\text { 3m,400 MHz, } \mathrm{CDCl}_{3}
$$

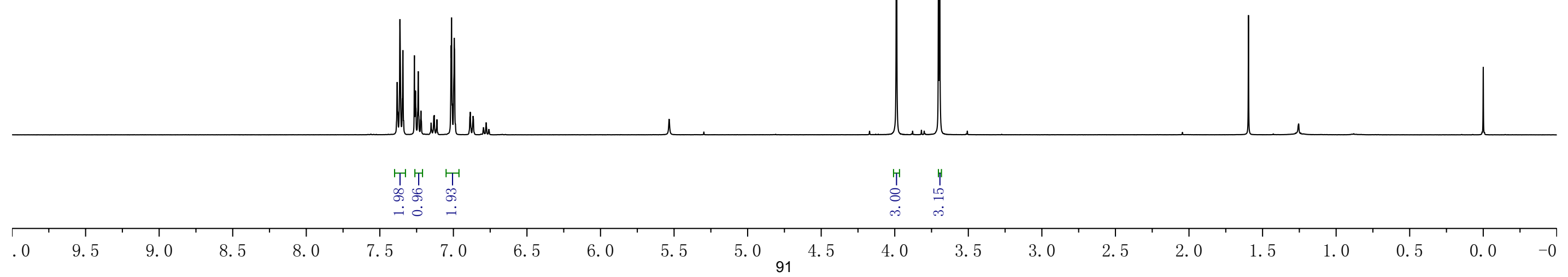


8 f
id
iी

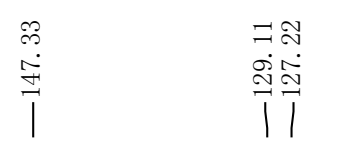

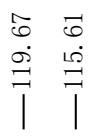

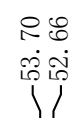

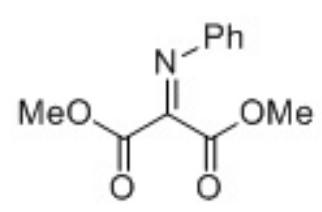

3m,100 MHz, $\mathrm{CDCl}_{3}$
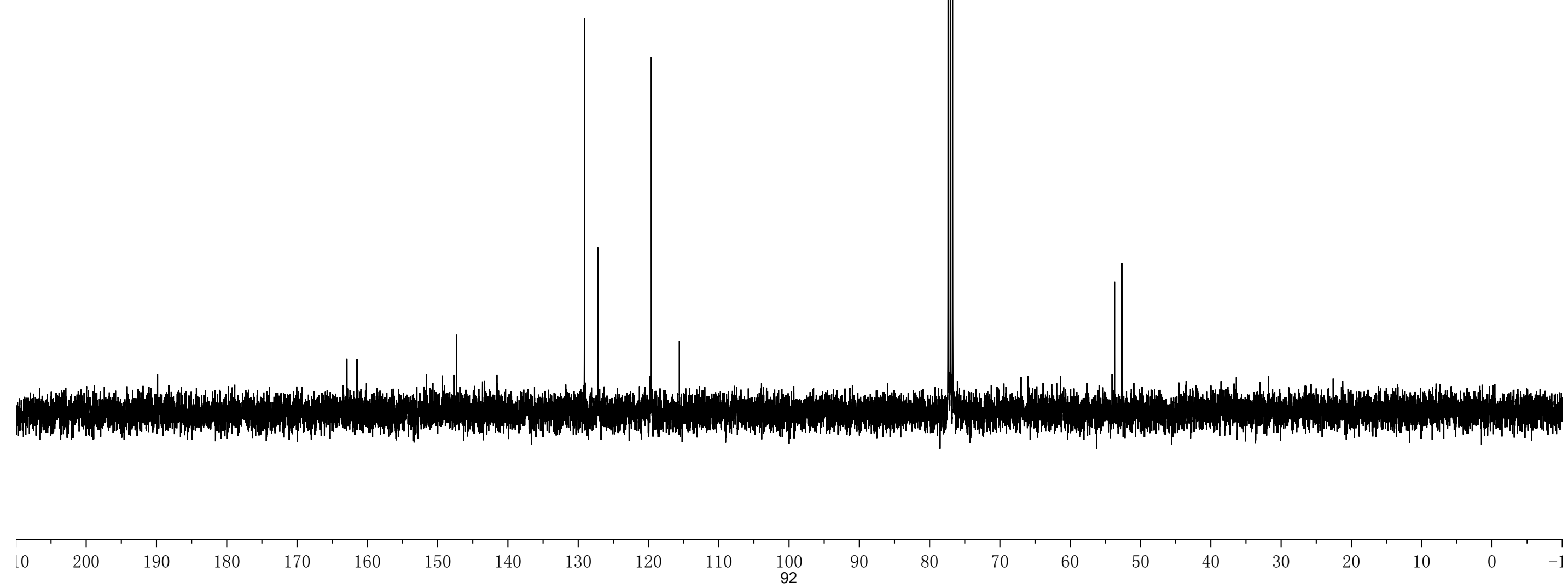


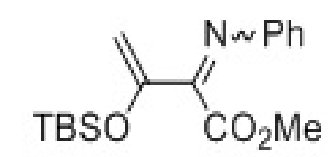

3n, $400 \mathrm{MHz}, \mathrm{CDCl}_{3}$

mixture of two geometric isomers (95:5)

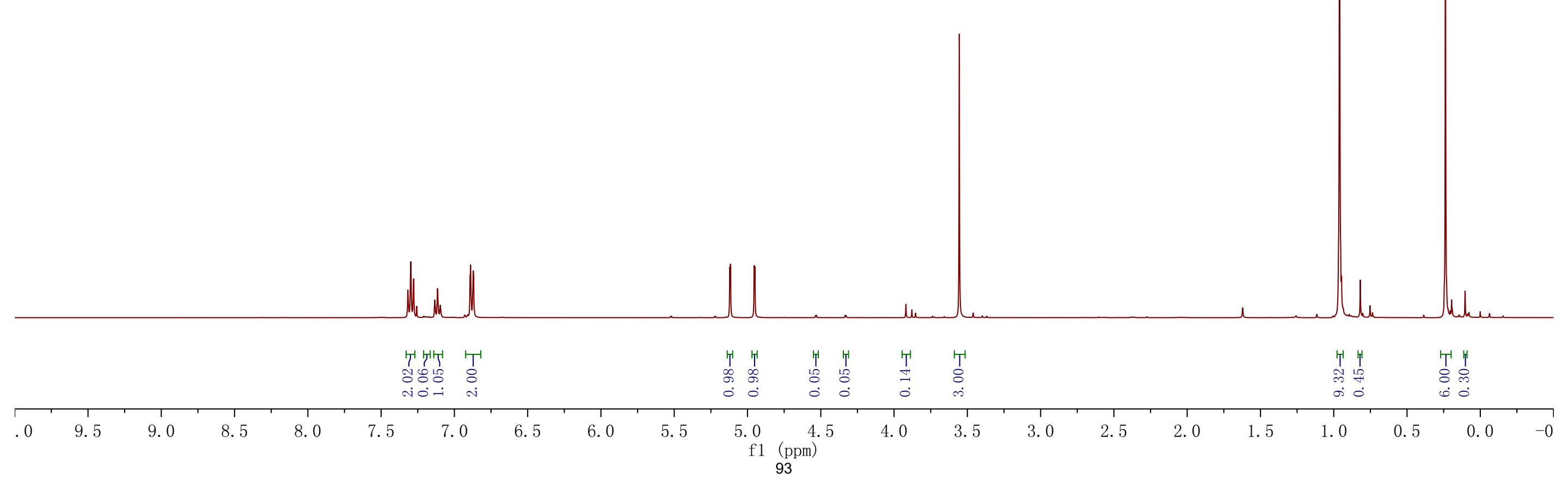




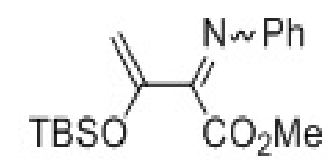

3n, $100 \mathrm{MHz}, \mathrm{CDCl}_{3}$

mixture of two geometric isomers (95:5)

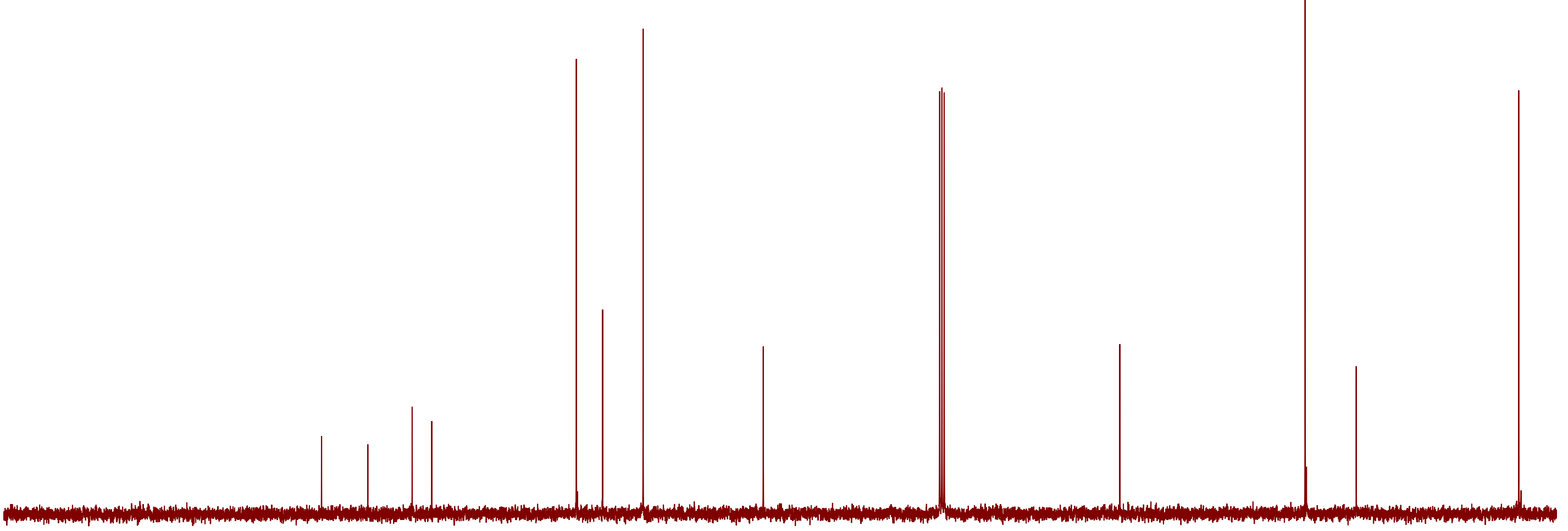

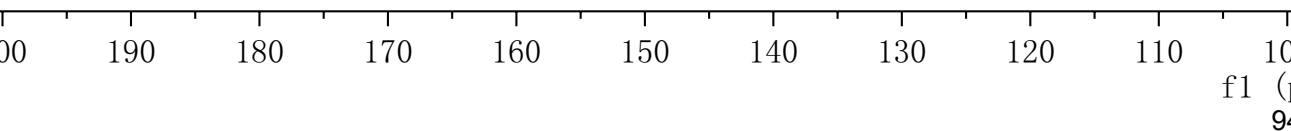


3o, $400 \mathrm{MHz}, \mathrm{CDCl}_{3}$

mixture of two geometric isomers (97:3)

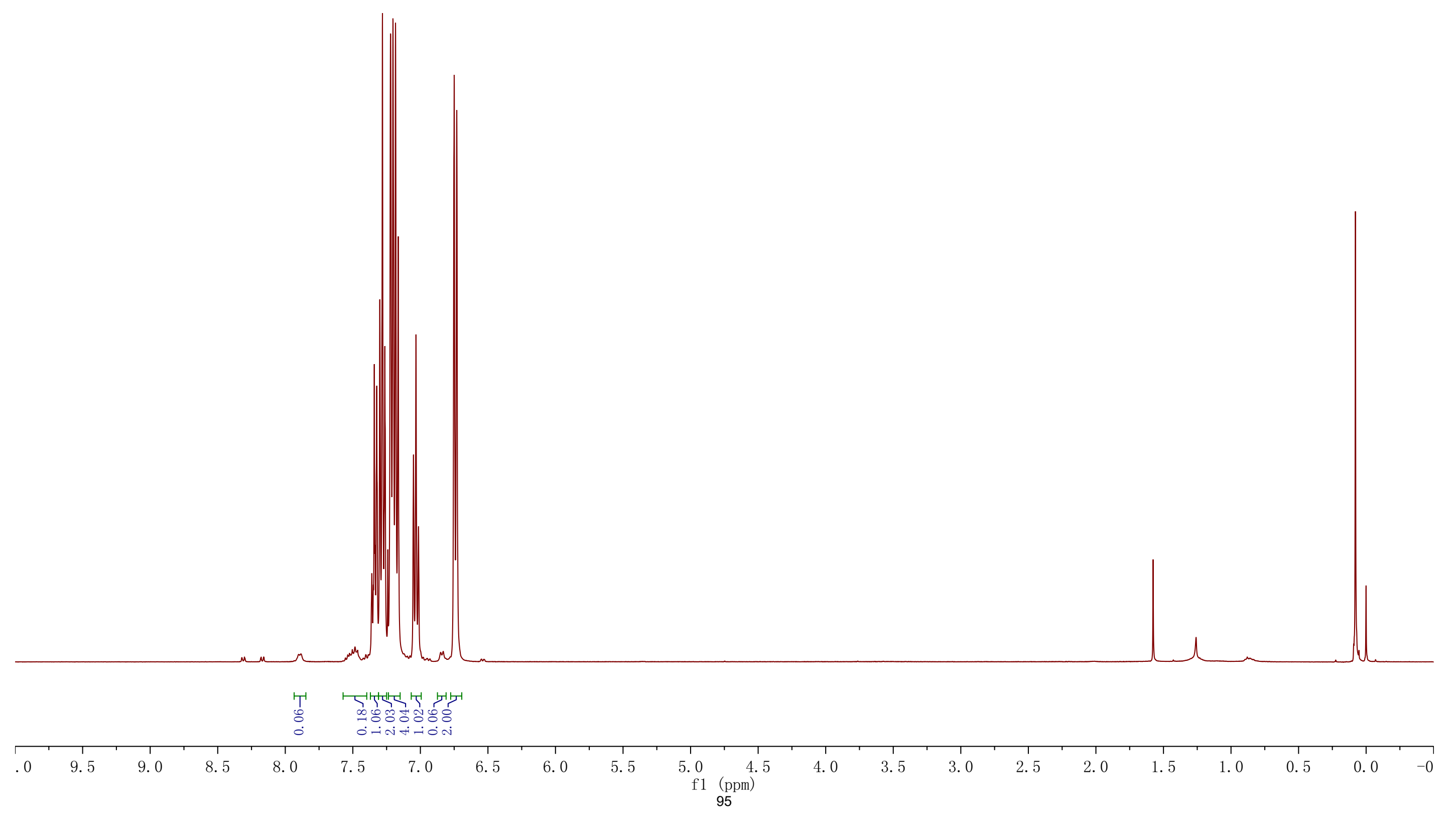




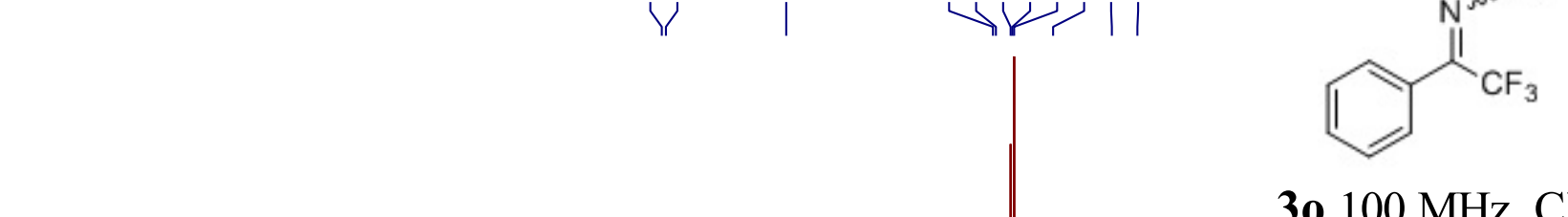

3o, $100 \mathrm{MHz}, \mathrm{CDCl}_{3}$

mixture of two geometric isomers (97:3) 


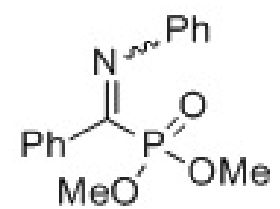

3p, $400 \mathrm{MHz}, \mathrm{CDCl}_{3}$

mixture of two geometric isomers $(90: 10)$

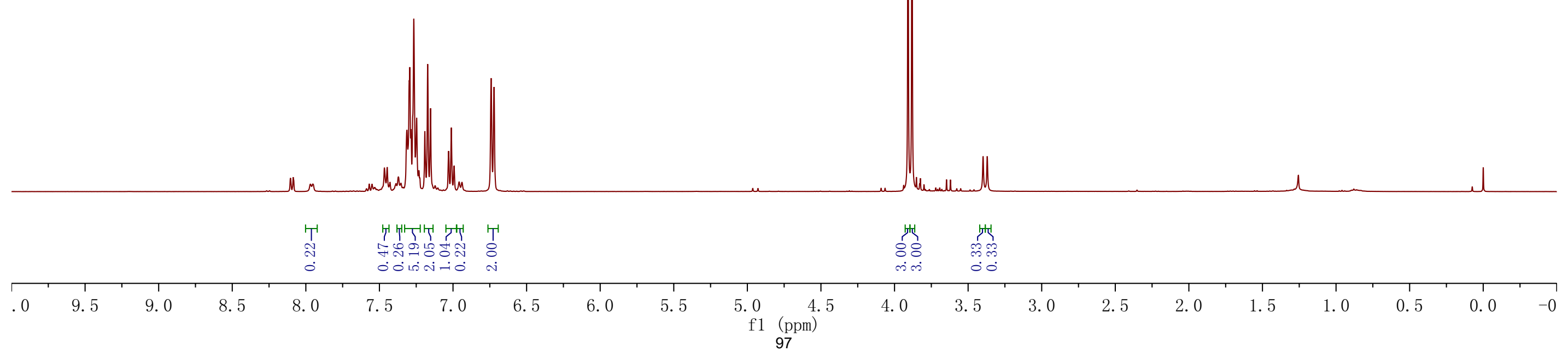




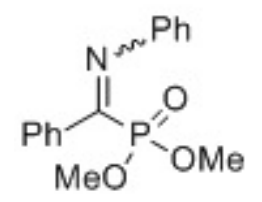

3p, $100 \mathrm{MHz}, \mathrm{CDCl}_{3}$

mixture of two geometric isomers (90:10)

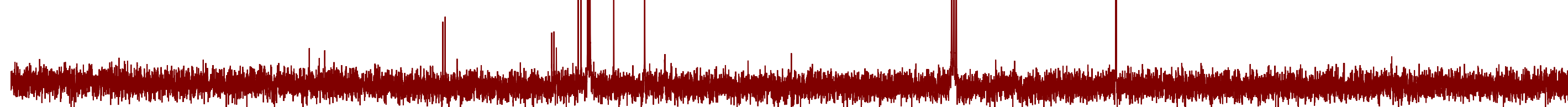

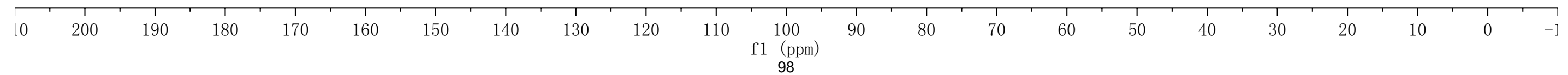




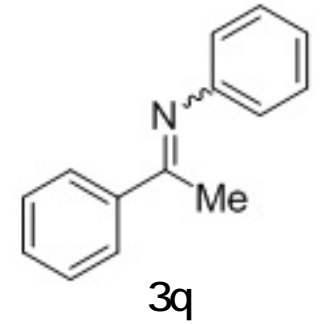

$400 \mathrm{MHz}, \mathrm{DMSO}_{-} \mathrm{d}_{6}$

mixture of two geometric isomers (87:13)

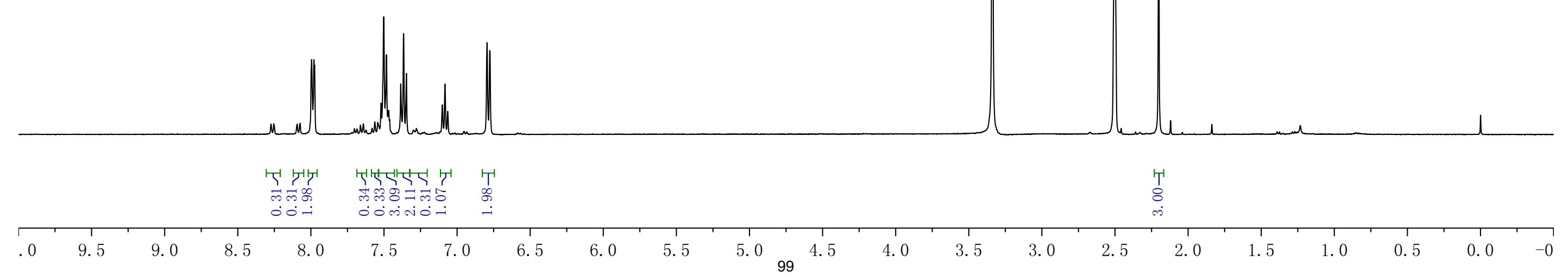




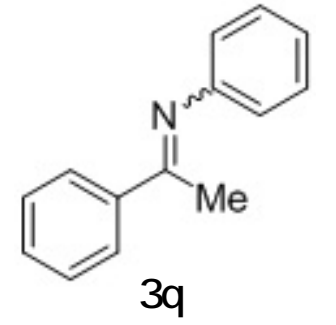

$75 \mathrm{MHz}, \mathrm{DMSO}_{6} \mathrm{~d}_{6}$

mixture of two geometric isomers (87:13)

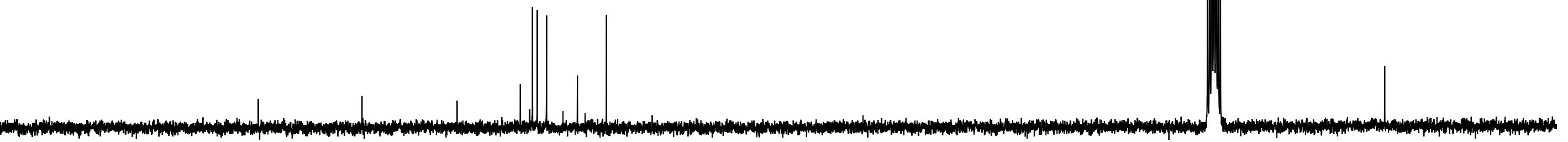




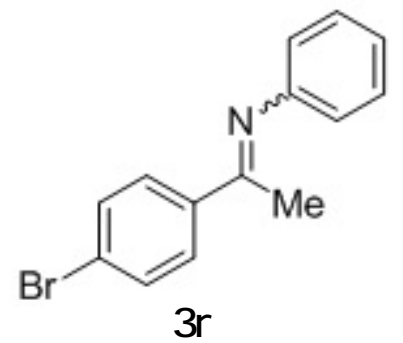

$300 \mathrm{MHz}, \mathrm{DMSO}-\mathrm{d}_{6}$

mixture of two geometric isomers (93:7)

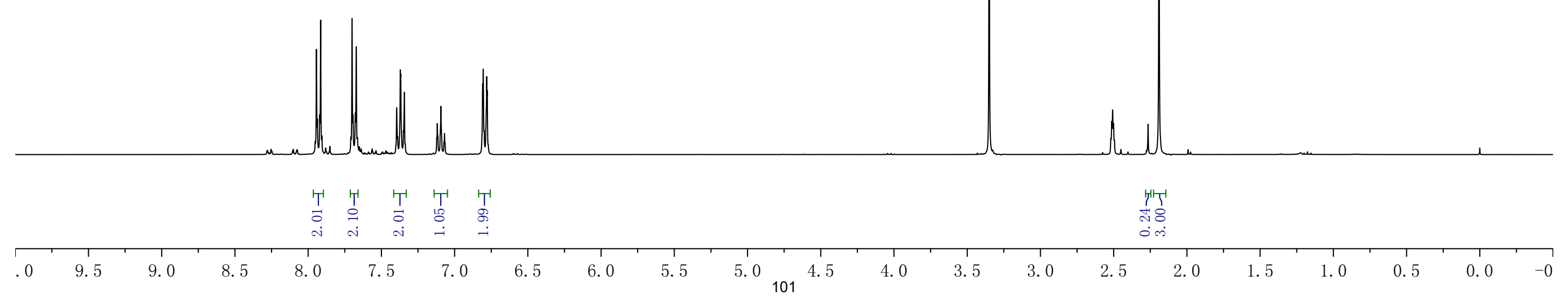




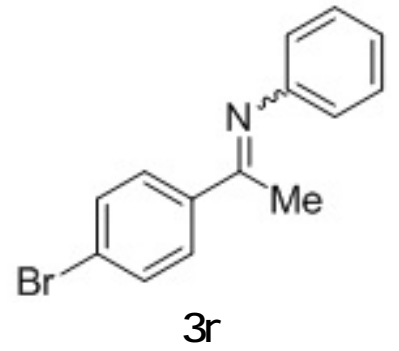

$75 \mathrm{MHz}, \mathrm{DMSO}^{-\mathrm{d}_{6}}$

mixture of two geometric isomers (93:7)

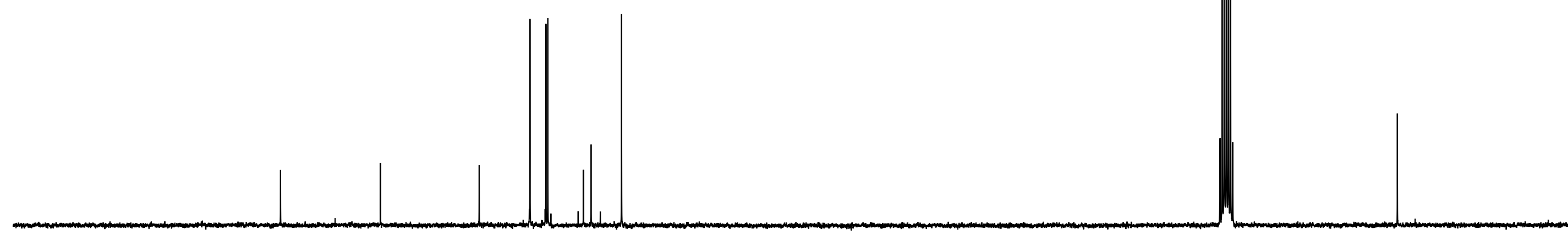




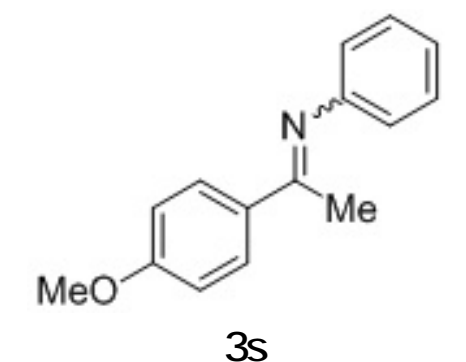

$300 \mathrm{MHz}$, DMSO-d 6

mixture of two geometric isomers (91:9)

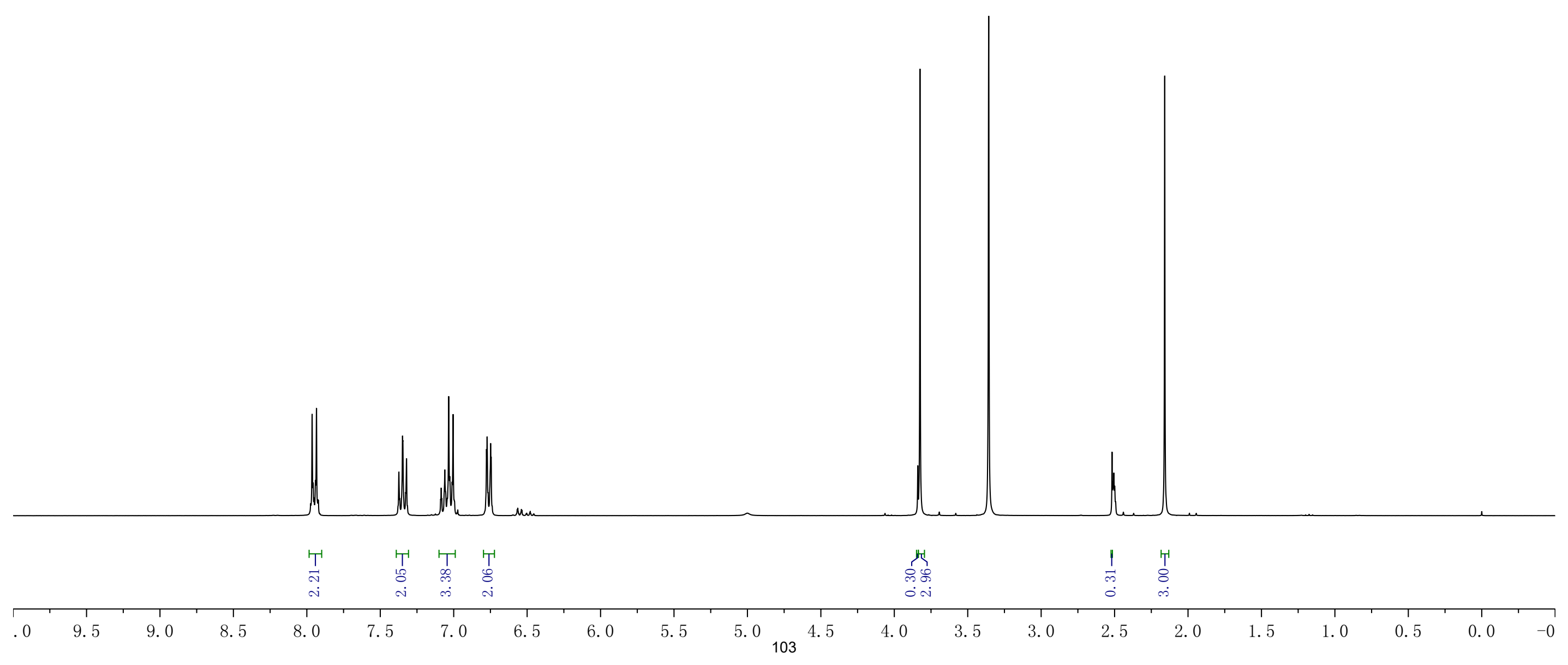




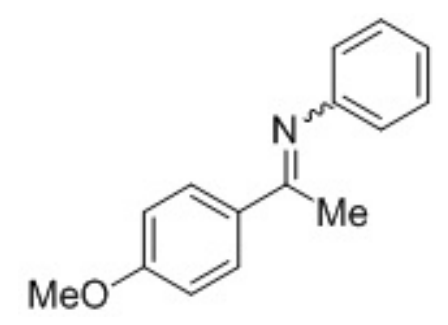

3s

$75 \mathrm{MHz}, \mathrm{DMSO}^{-\mathrm{d}_{6}}$

mixture of two geometric isomers (91:9)

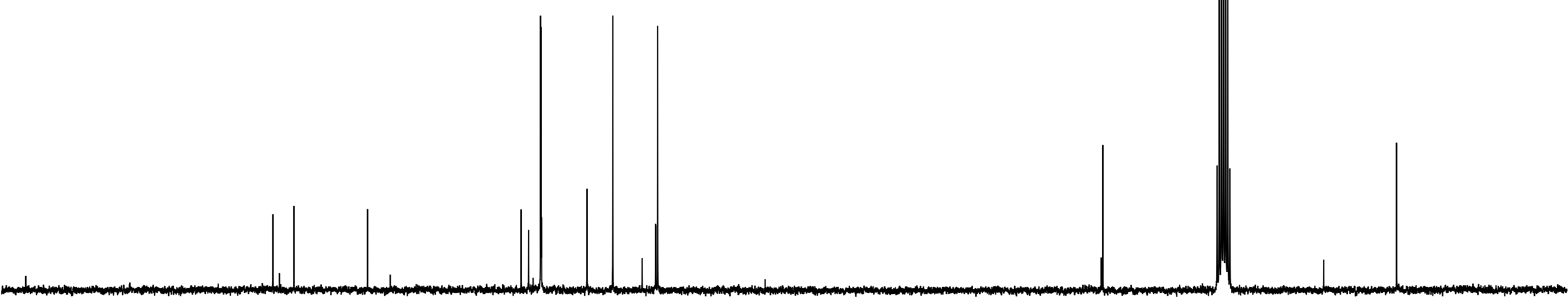




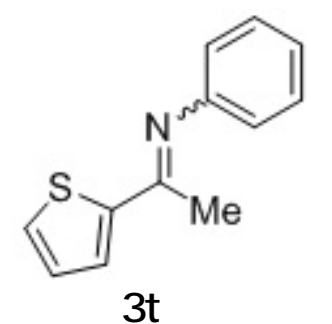

$400 \mathrm{MHz}, \mathrm{DMSO}_{-} \mathrm{d}_{6}$

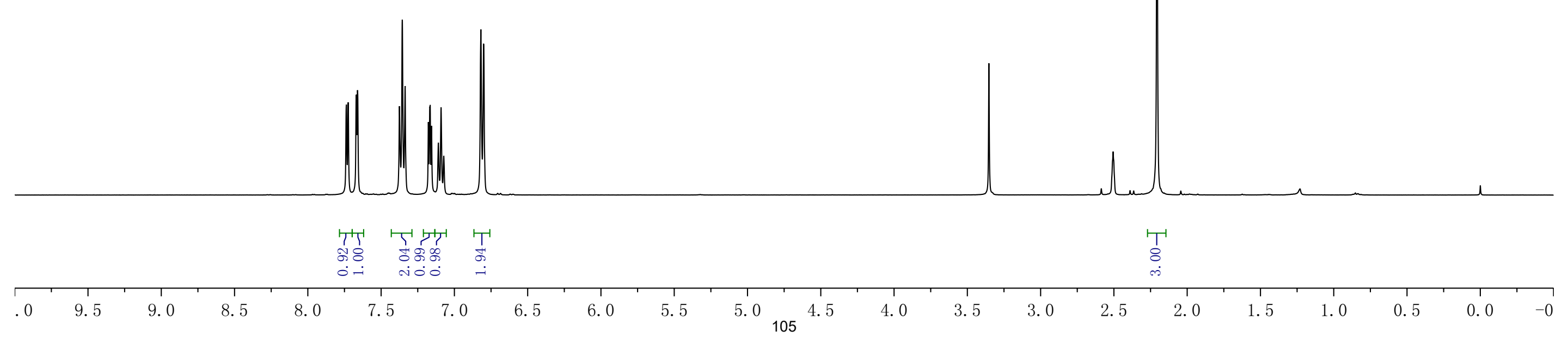




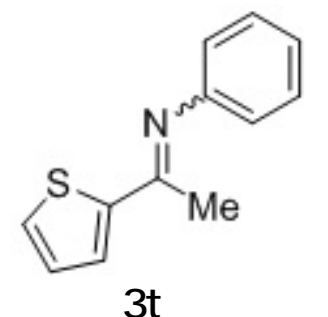

$75 \mathrm{MHz}, \mathrm{DMSO}_{-} \mathrm{d}_{6}$

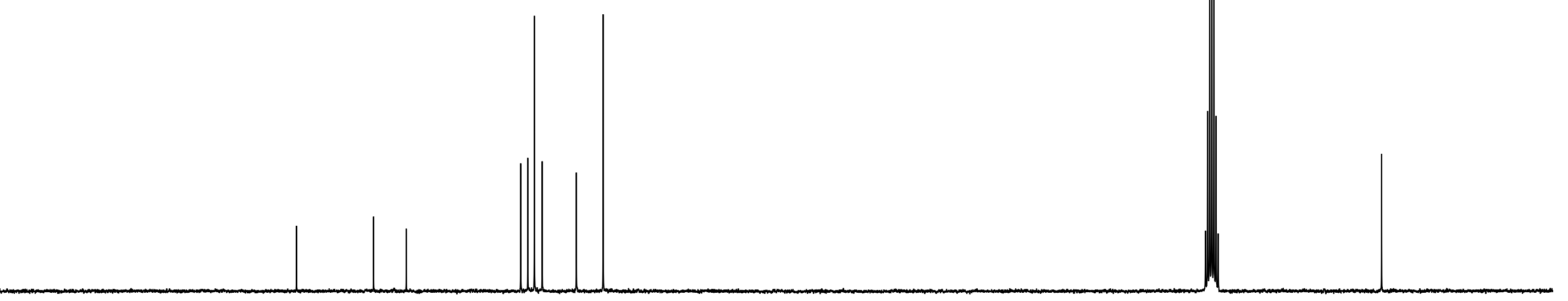




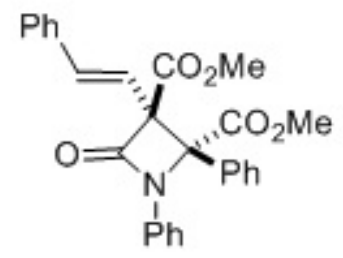

5a, $400 \mathrm{MHz}, \mathrm{CDCl}_{3}$
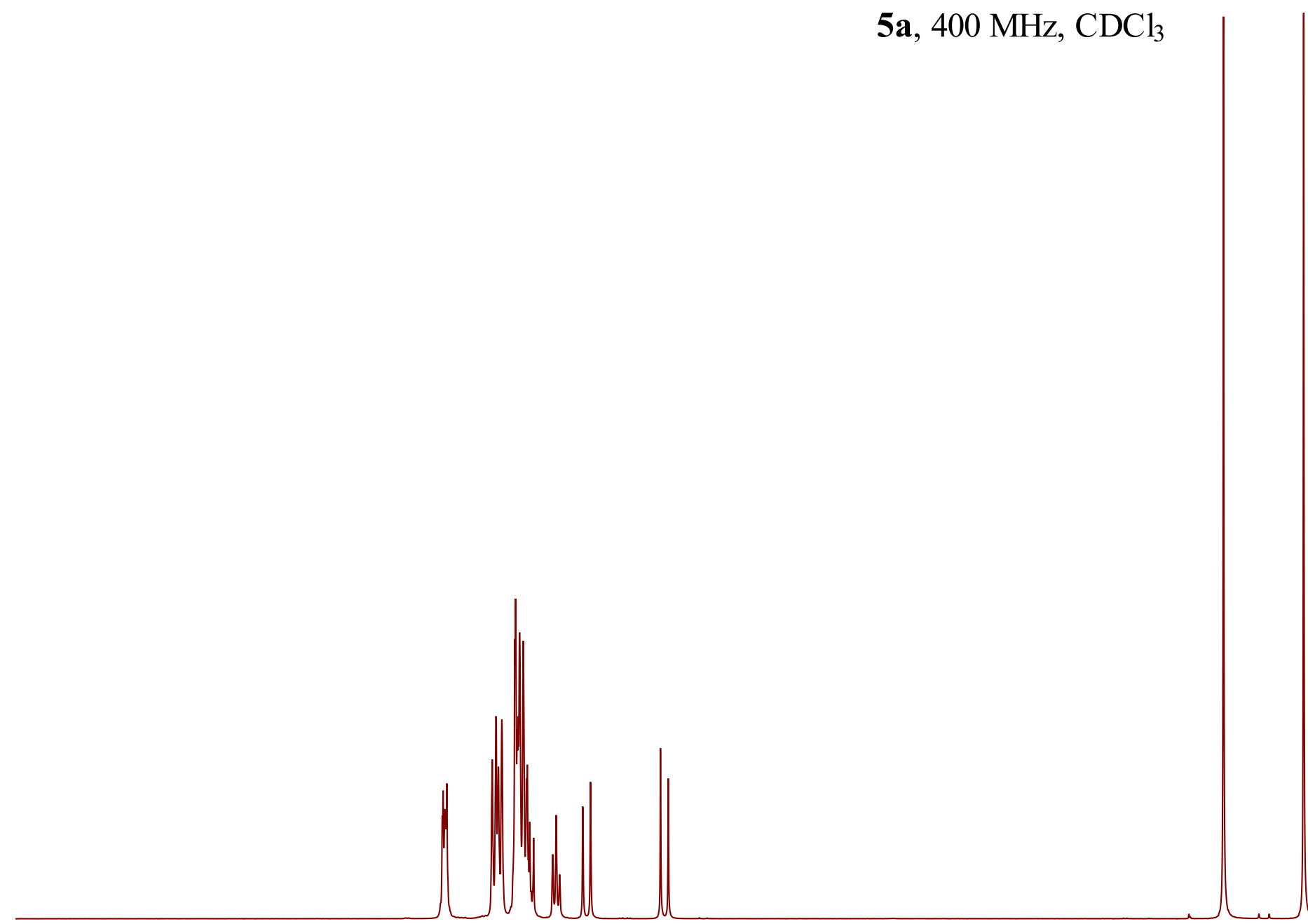

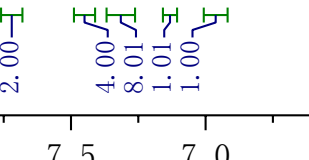

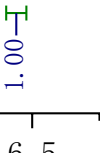




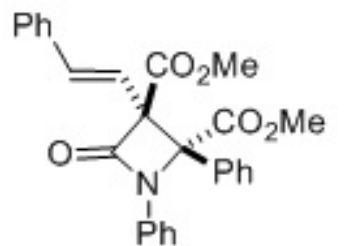

5a, $100 \mathrm{MHz}, \mathrm{CDCl}_{3}$

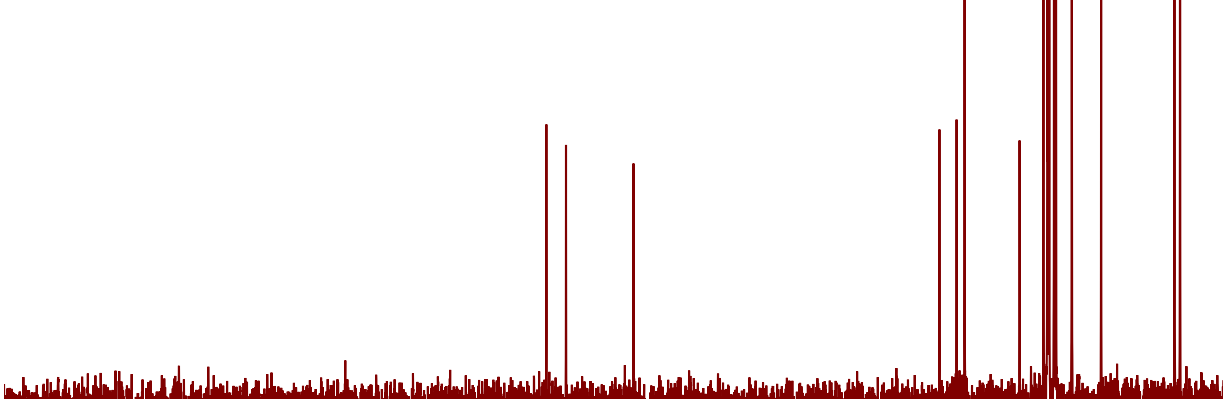

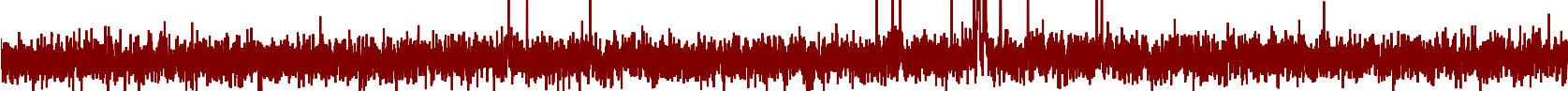
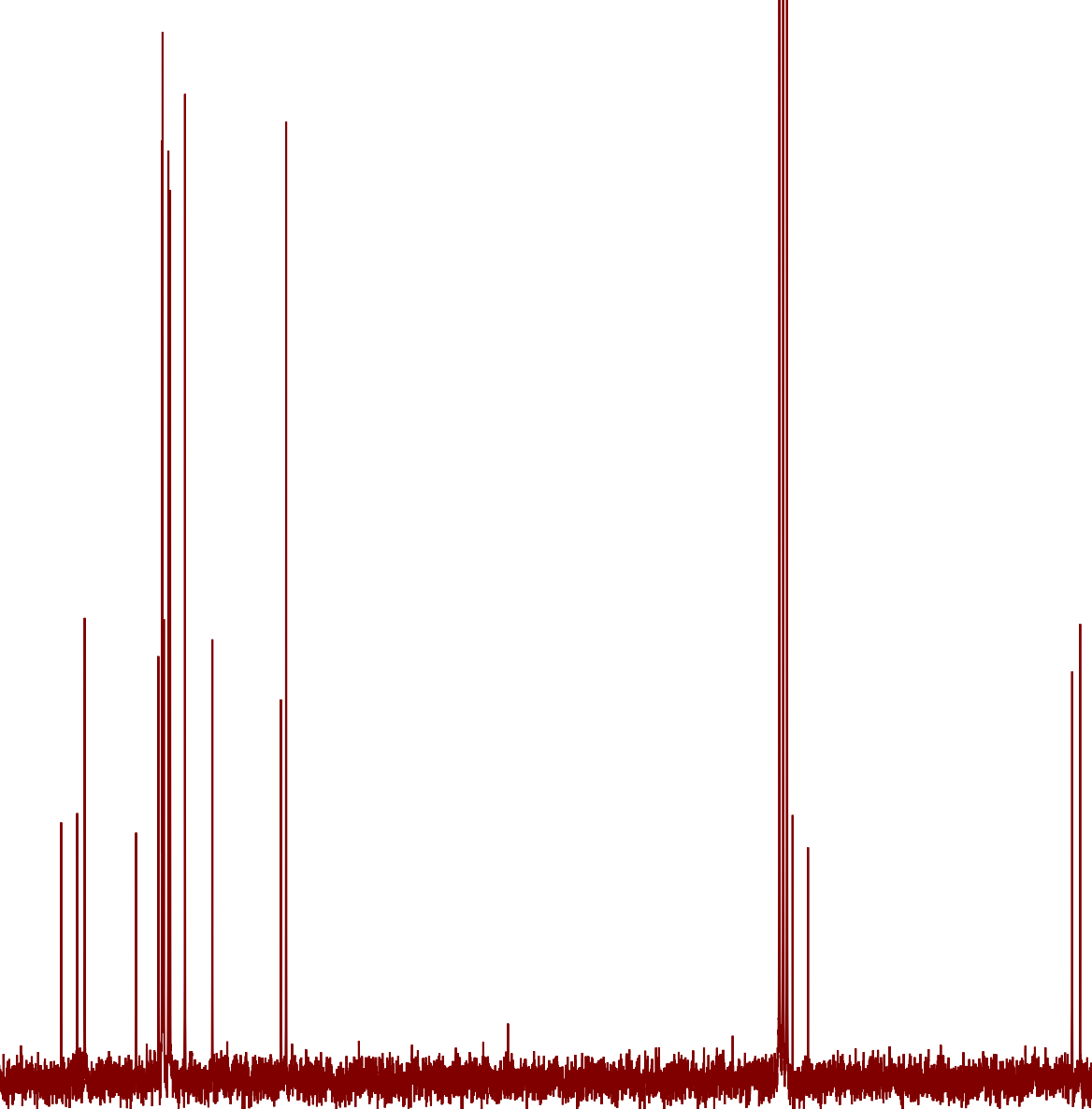


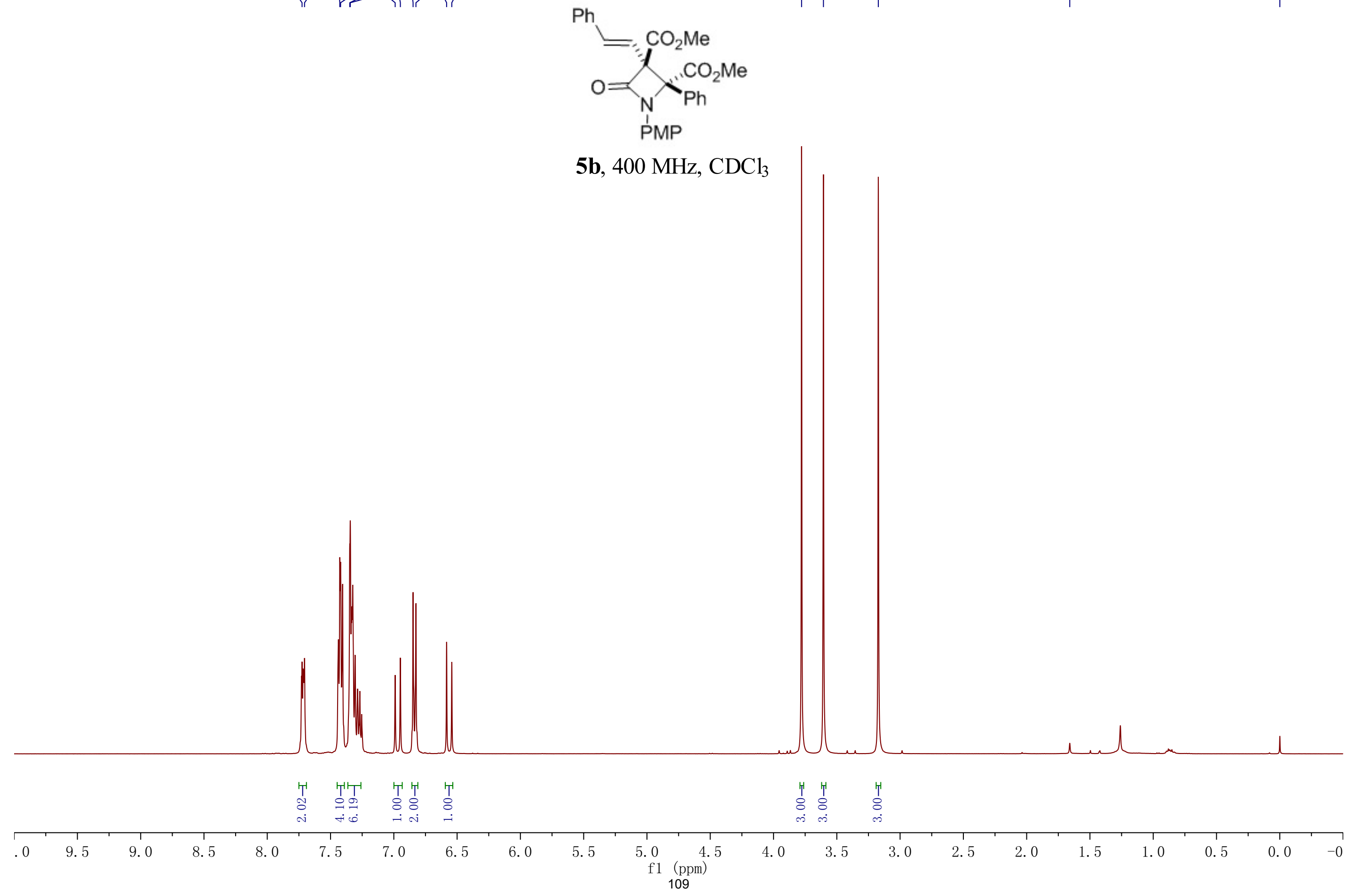


$\mathrm{Ph}$

$$
=\overbrace{\mathrm{PMP}}^{\mathrm{CO}_{2} \mathrm{Me}}
$$

5b, $100 \mathrm{MHz}, \mathrm{CDCl}_{3}$
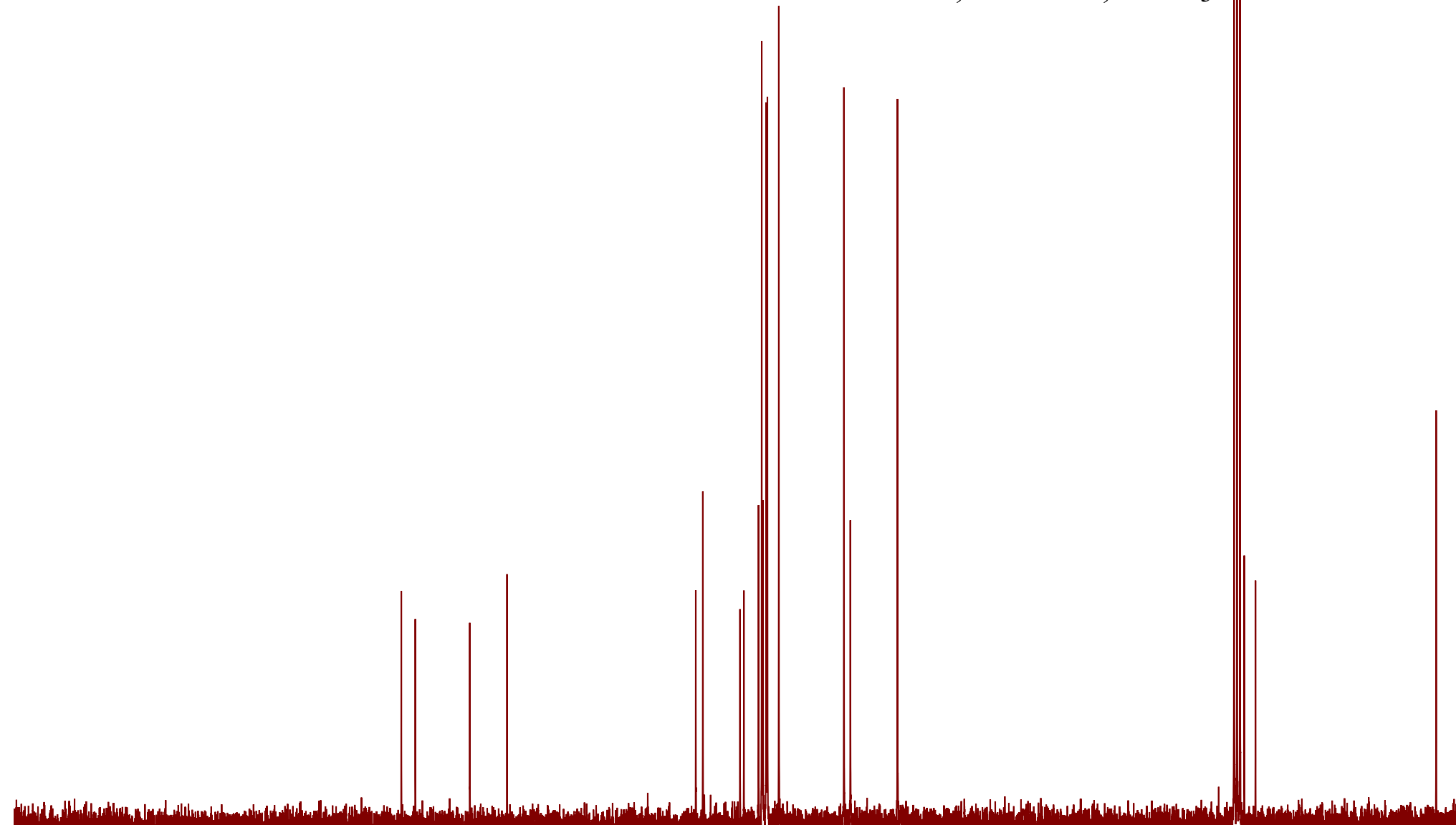

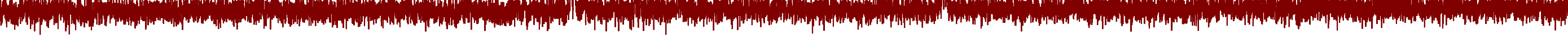

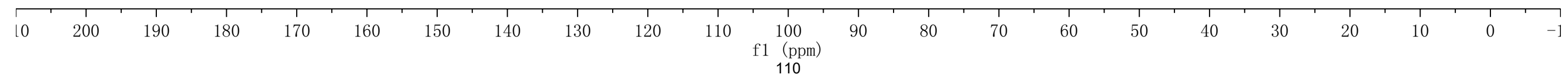




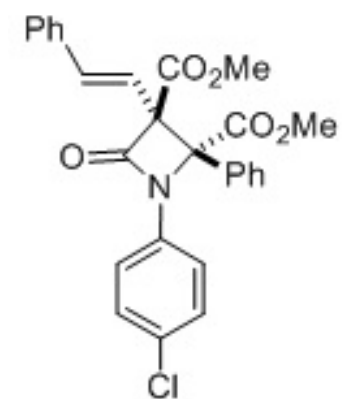

5c, $400 \mathrm{MHz}, \mathrm{CDCl}_{3}$

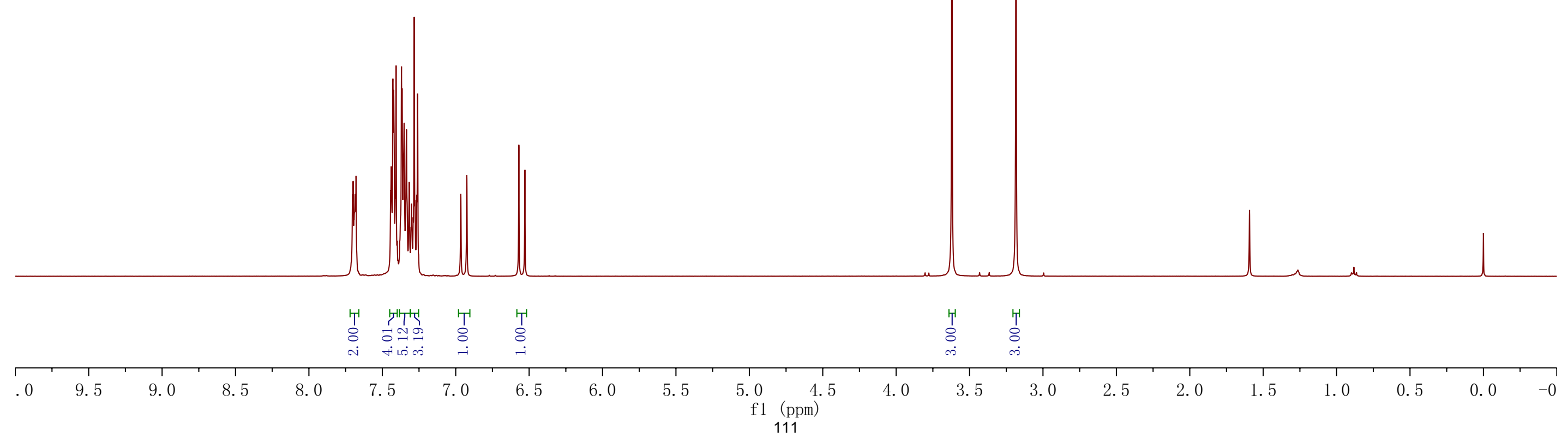



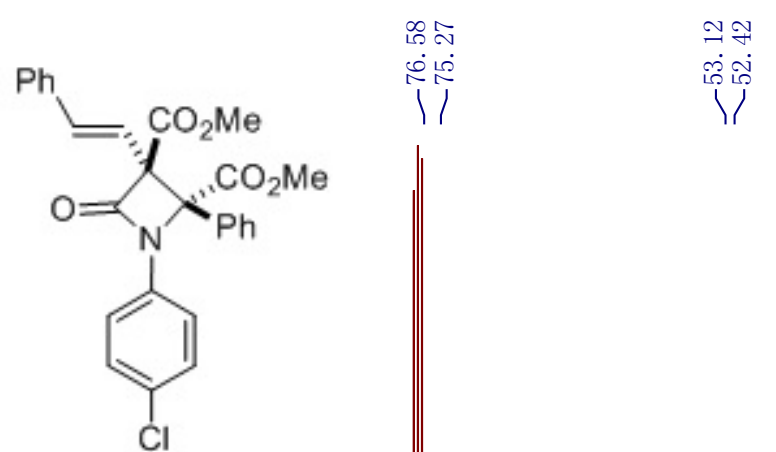

5c, $100 \mathrm{MHz}, \mathrm{CDCl}_{3}$

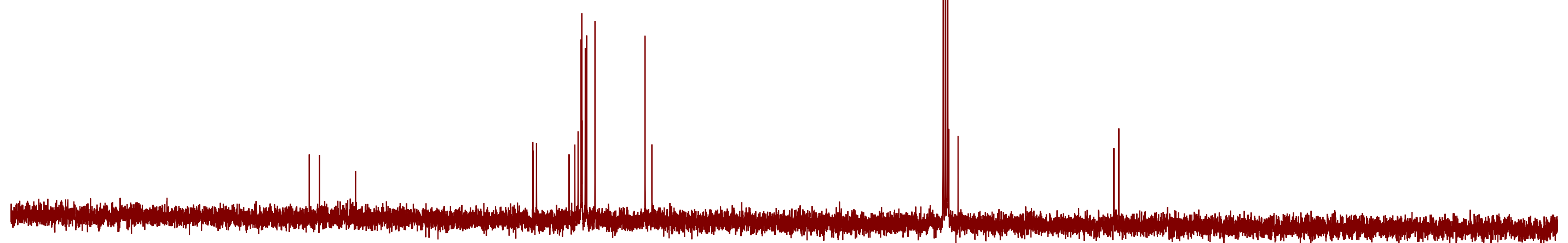

$\begin{array}{rr}190 & 180\end{array}$

170

160

150

140

130

120

110

100

$\begin{array}{ll}1 & 1 \\ 90 \quad 80\end{array}$ 
$\mathrm{Ph}$

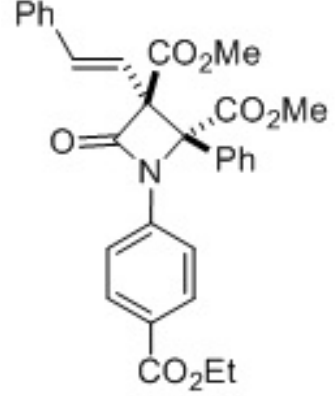

5d, $400 \mathrm{MHz}, \mathrm{CDCl}_{3}$

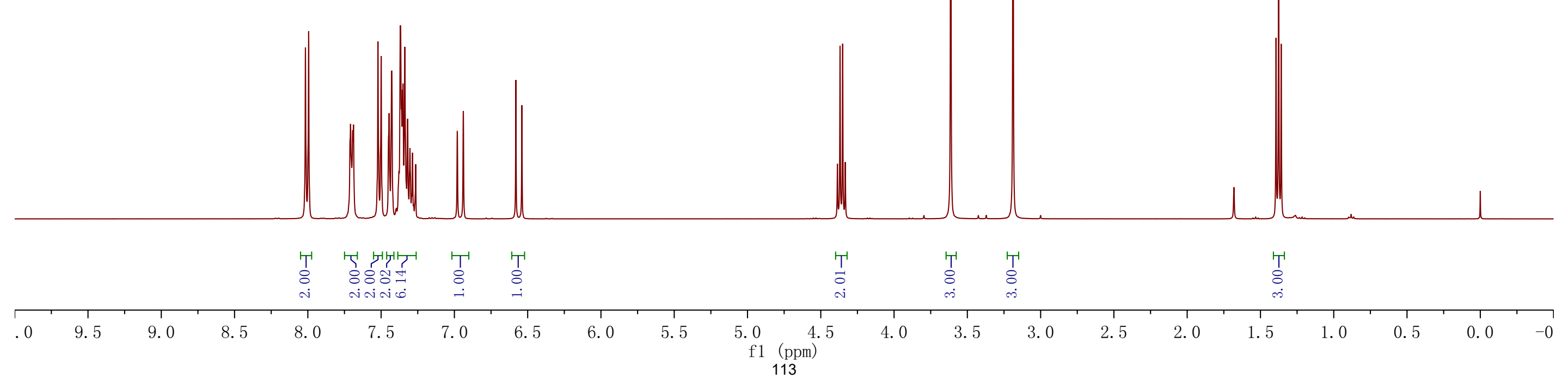




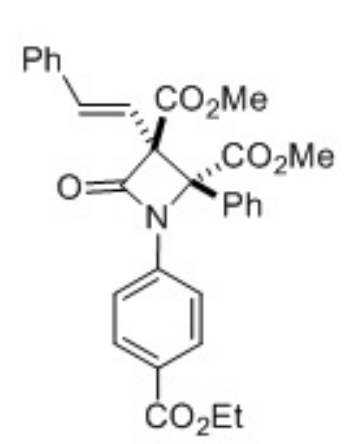

5d, $100 \mathrm{MHz}, \mathrm{CDCl}_{3}$

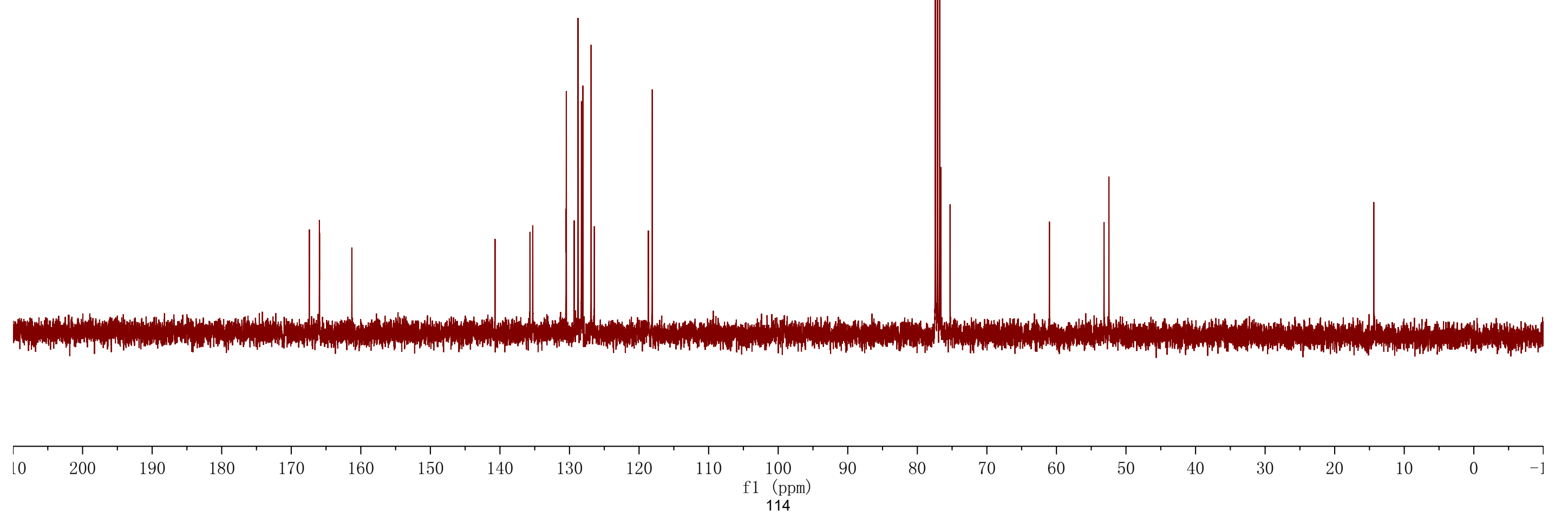




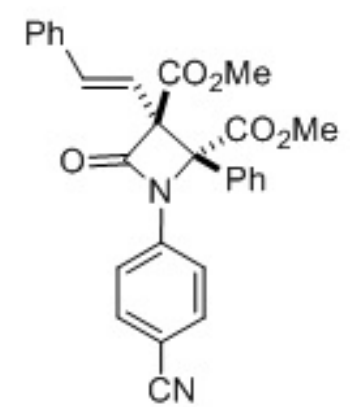

5e, $400 \mathrm{MHz}, \mathrm{CDCl}_{3}$

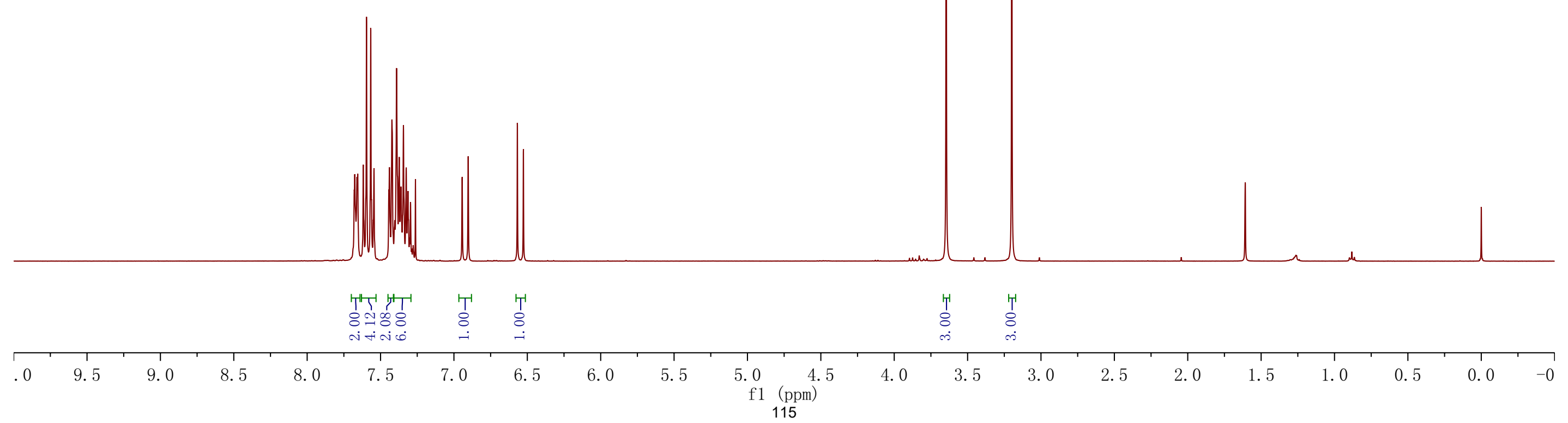




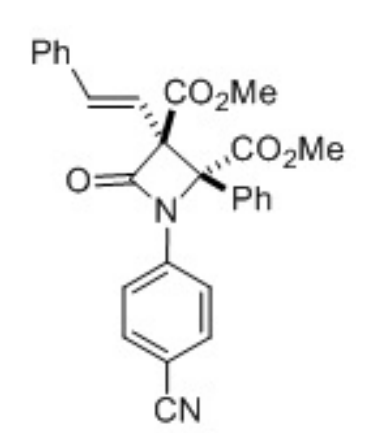

5e, $100 \mathrm{MHz}, \mathrm{CDCl}_{3}$

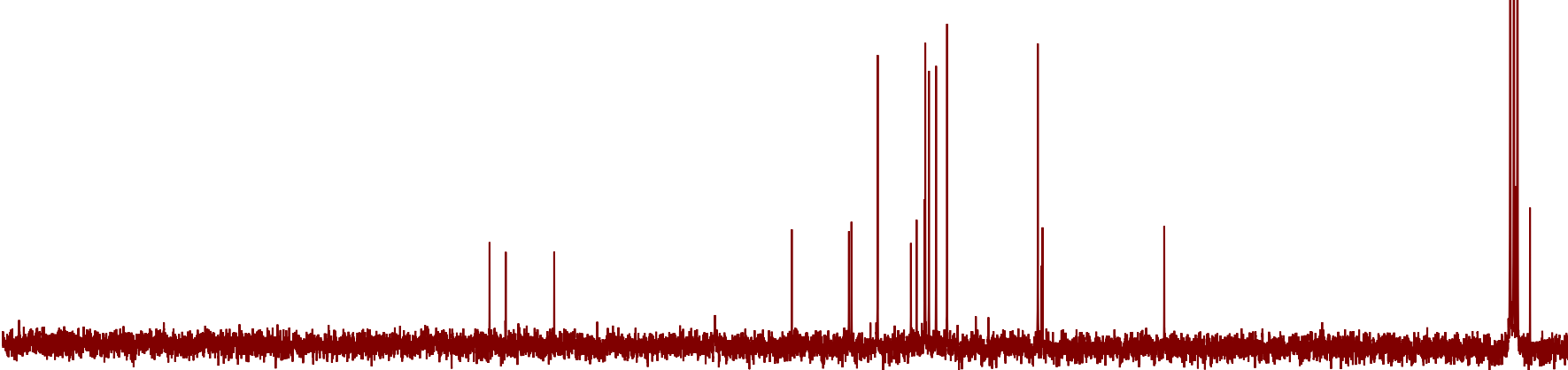




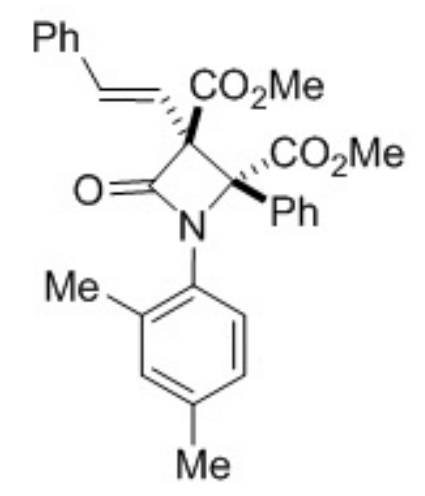

5f, $400 \mathrm{MHz}, \mathrm{CDCl}_{3}$

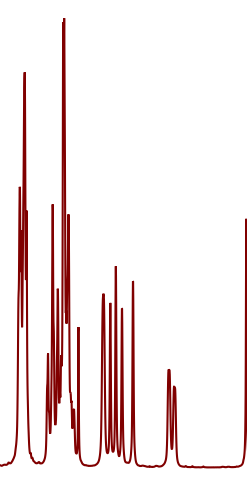

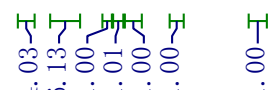

$\begin{array}{ll}T & T \\ 8 & 8 \\ 8 & 9\end{array}$

$\begin{array}{ll}1 & 1 \\ 0 & 8 \\ \dot{\infty} & \dot{n}\end{array}$ 


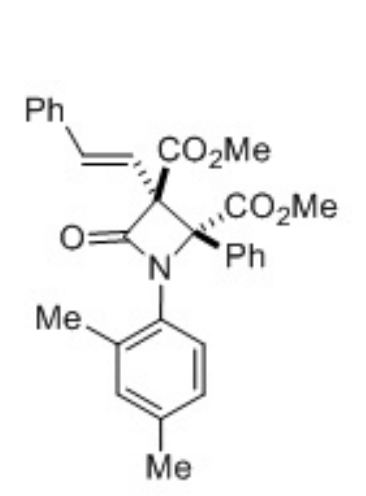

5f, $100 \mathrm{MHz}, \mathrm{CDCl}_{3}$

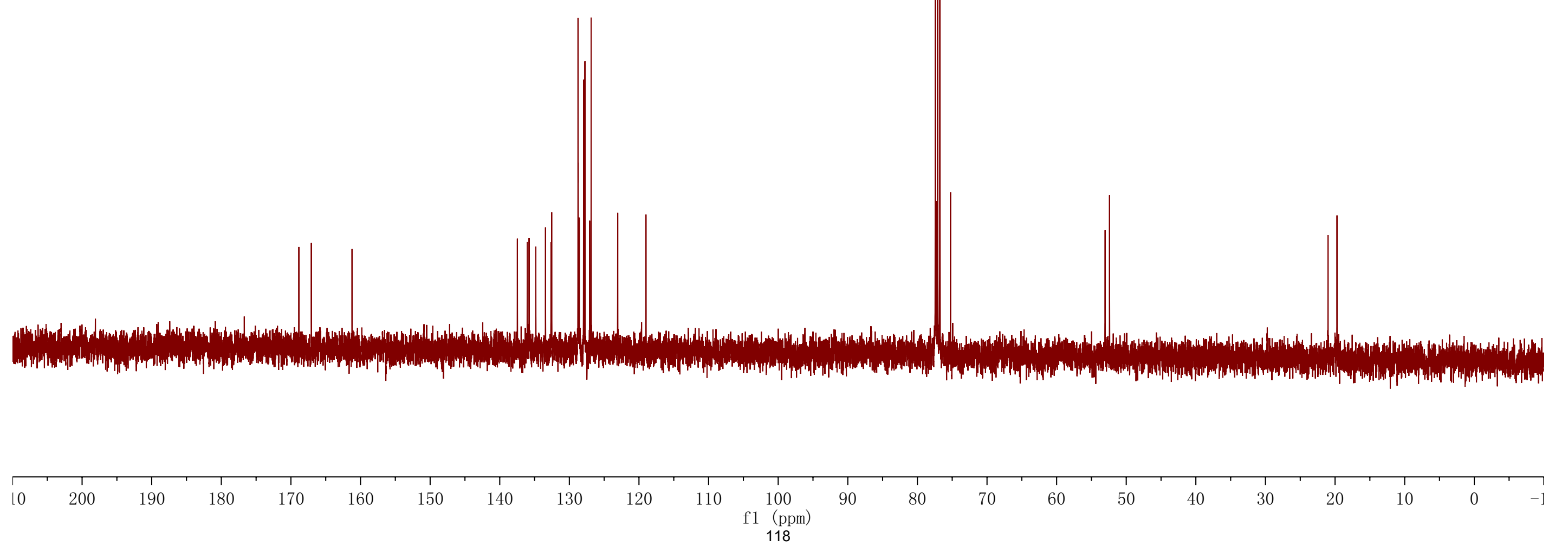




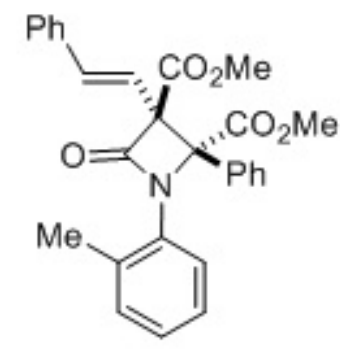

5g, $400 \mathrm{MHz}, \mathrm{CDCl}_{3}$

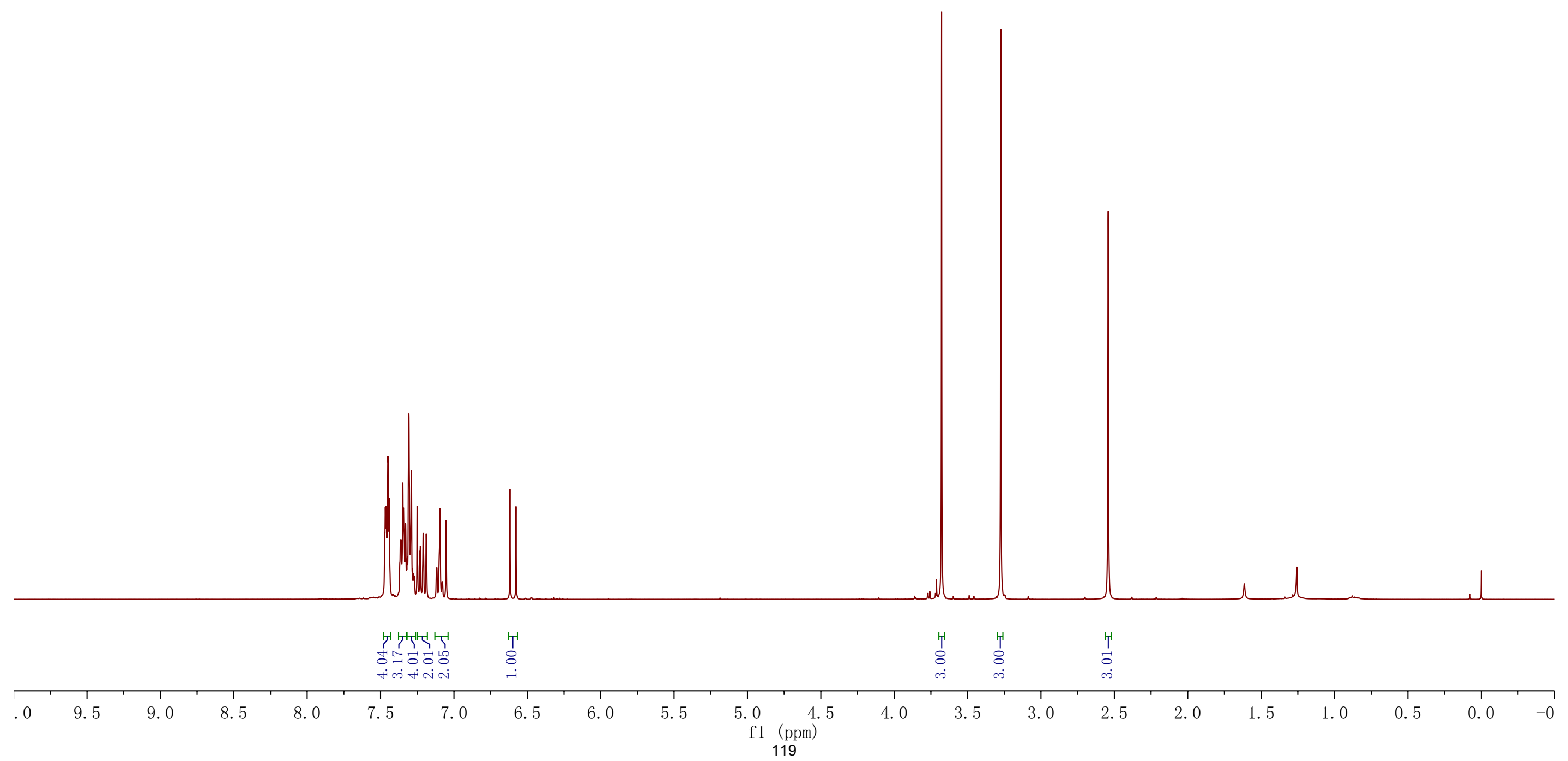


5g, $100 \mathrm{MHz}, \mathrm{CDCl}_{3}$

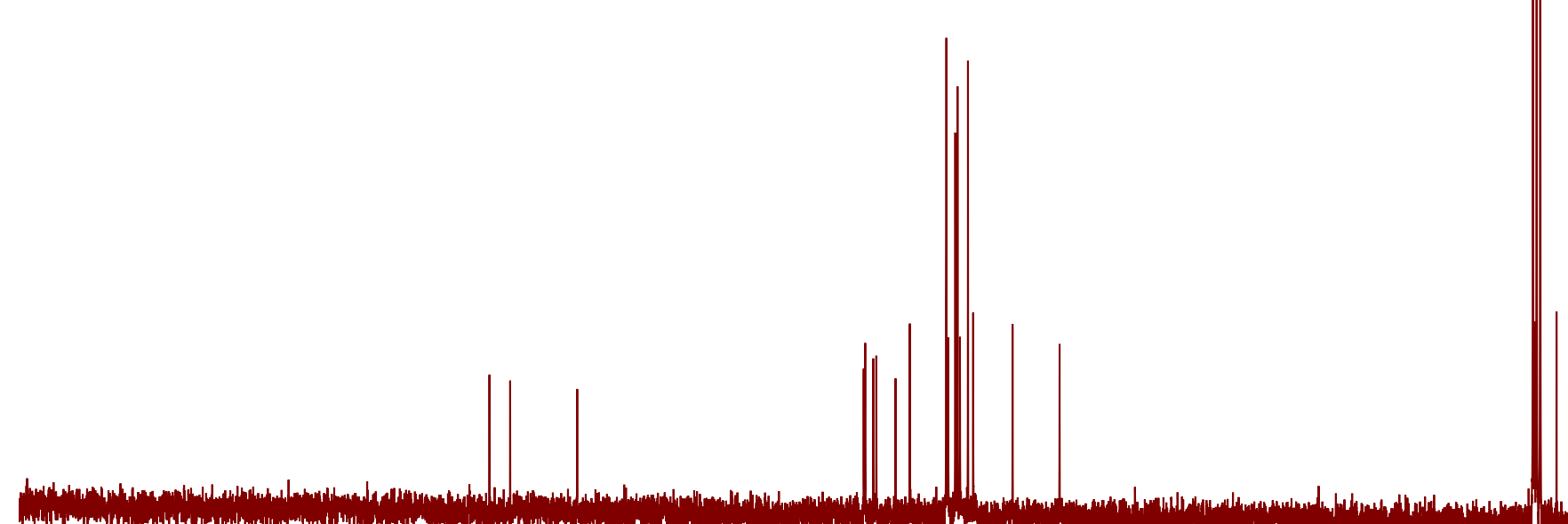

||

$\begin{array}{rrr}1 & 1 \\ 190 & 180\end{array}$

170

160

150

140

130

120

110

100

$(\mathrm{ppm})$
120 


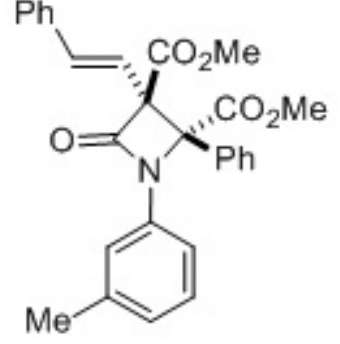

5h, $400 \mathrm{MHz}, \mathrm{CDCl}_{3}$

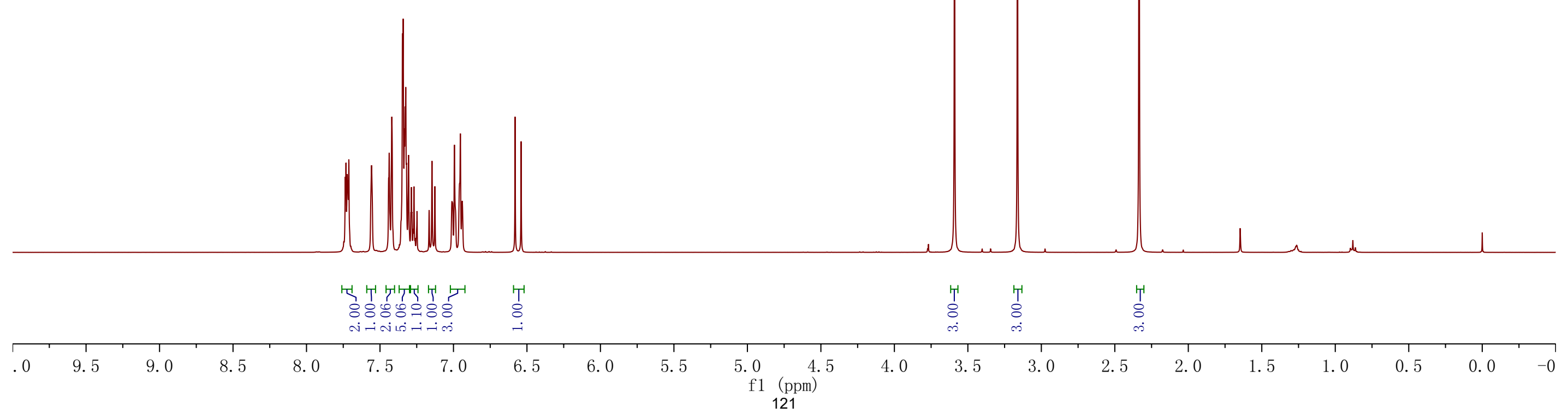



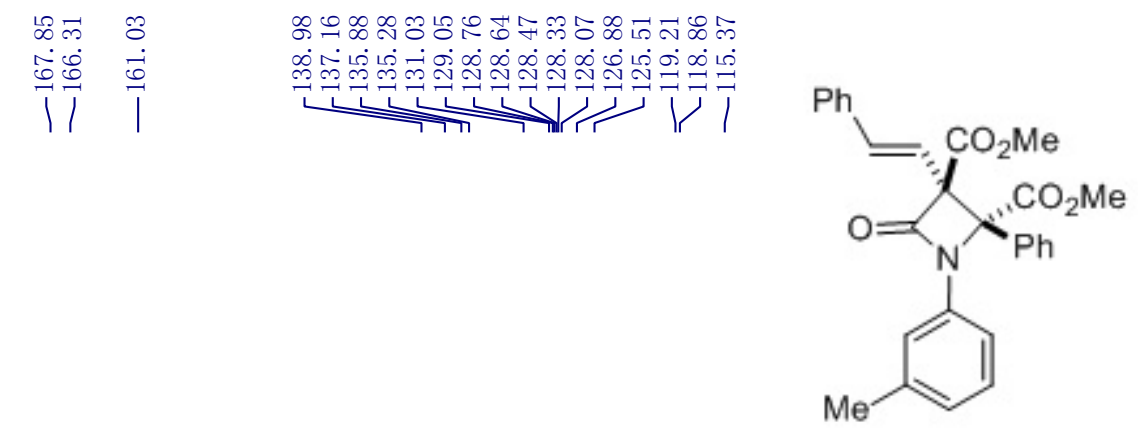

5h, $100 \mathrm{MHz}, \mathrm{CDCl}_{3}$ 
$\mathrm{Ph}$<smiles>C/C=C/[C@]1(C(=O)OC)C(=O)N(c2ccccc2)[C@]1(C(=O)OC)c1ccc(OC)cc1</smiles>

5i, $400 \mathrm{MHz}, \mathrm{CDCl}_{3}$

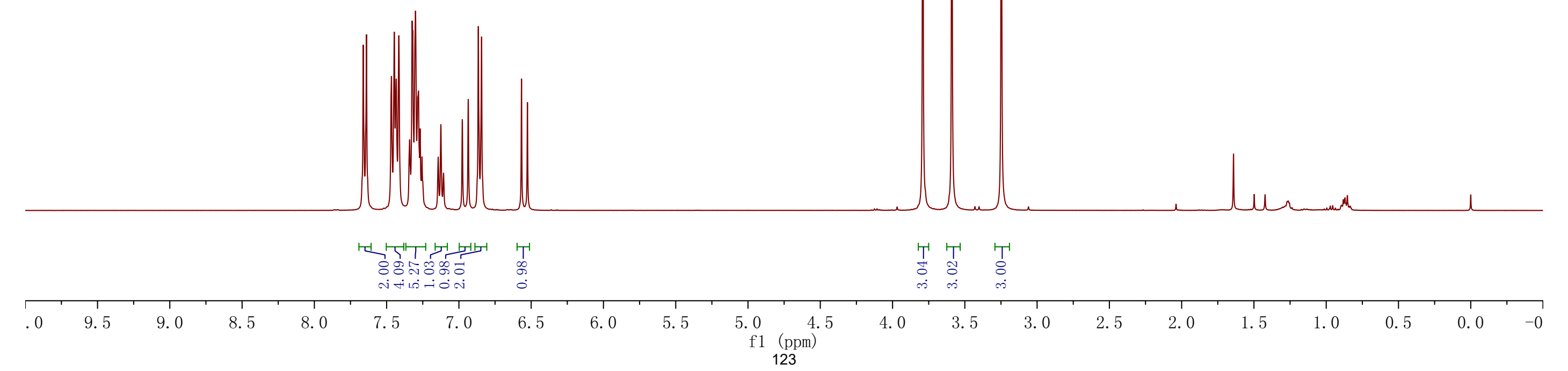


$\mathrm{Ph}$<smiles>COC(=O)C1(C(=O)OC)C(=O)N(P)[C@]1(C=CP)c1ccc(OC)cc1</smiles>

5i, $100 \mathrm{MHz}, \mathrm{CDCl}_{3}$

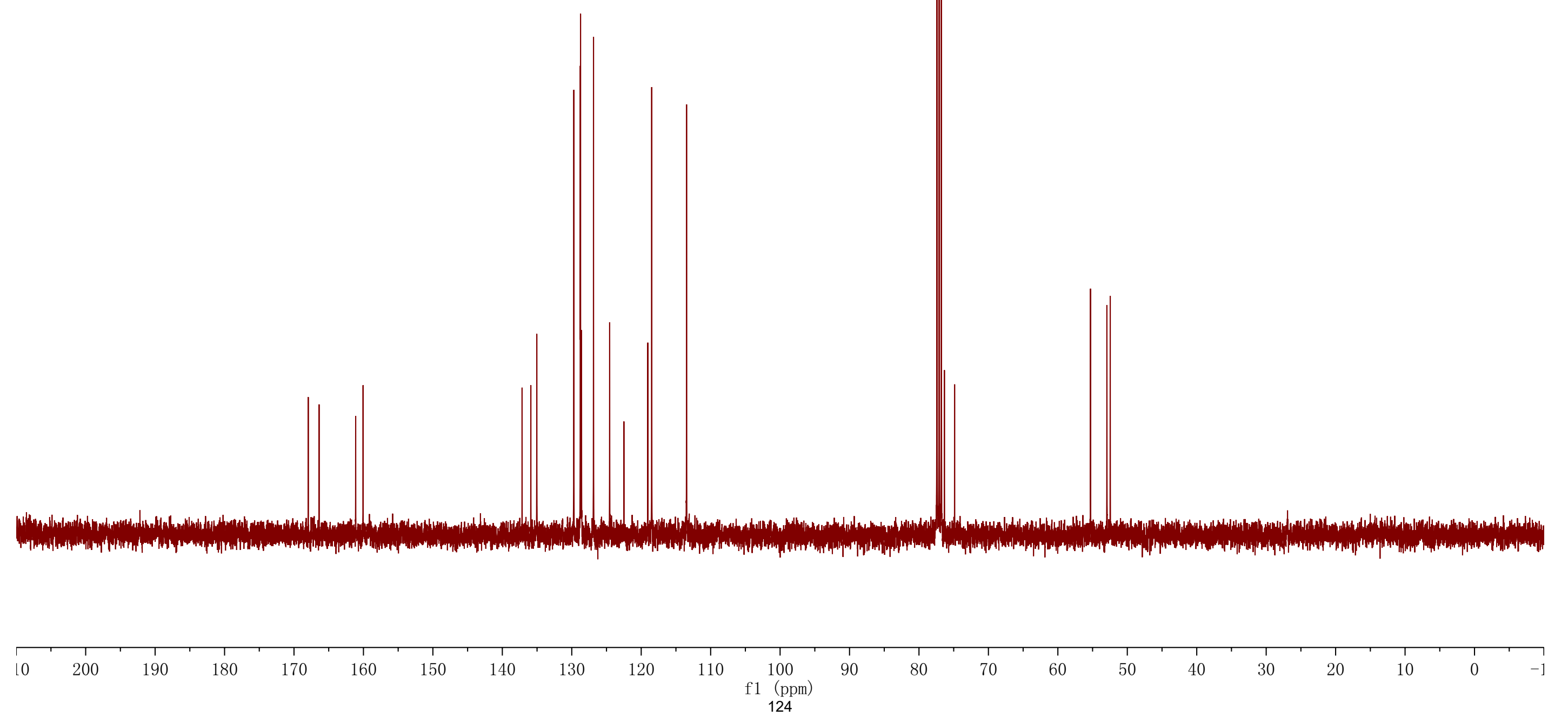


$\mathrm{Ph}$<smiles>COC(=O)[C@@]1(c2cccc(OC)c2)C(=O)N(c2ccccc2)[C@@]1(C=Cc1ccccc1)c1ccccc1</smiles>

5j, $400 \mathrm{MHz}, \mathrm{CDCl}_{3}$

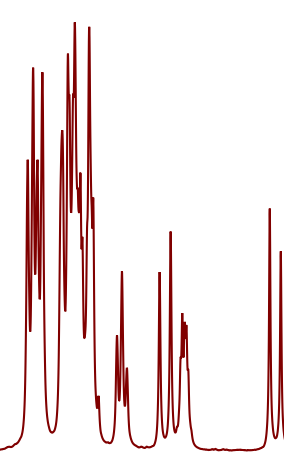

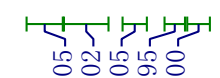

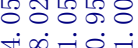

\section{J, $400 \mathrm{MHz}, \mathrm{CDCl}_{3}$}
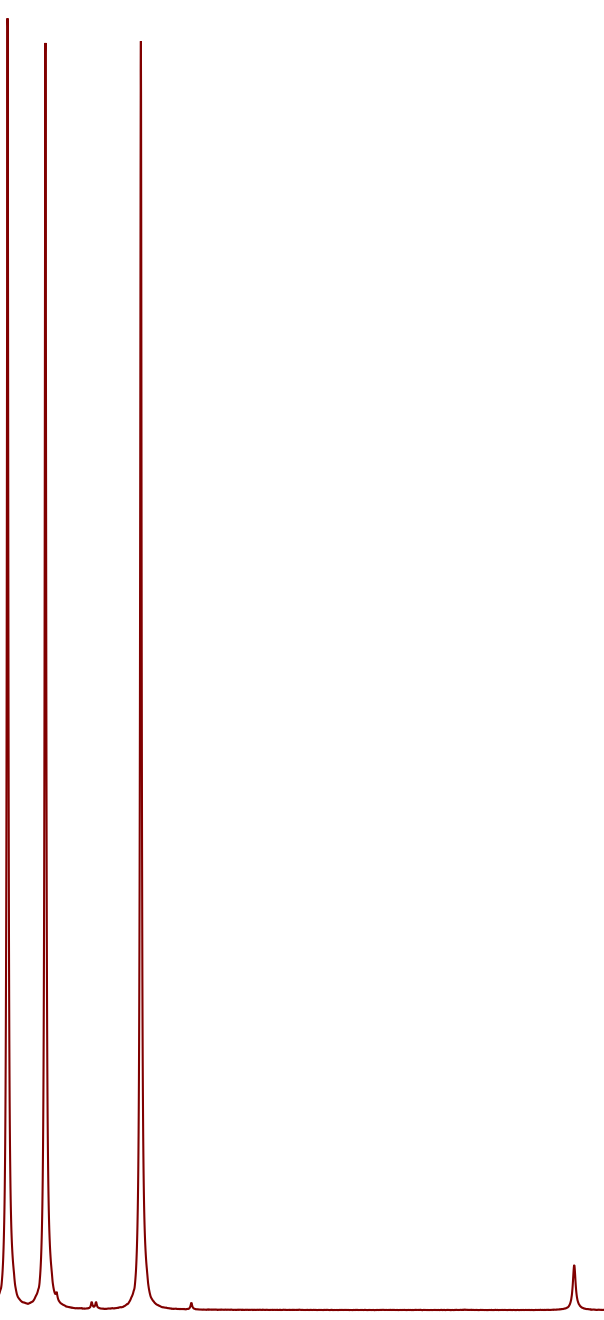

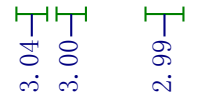

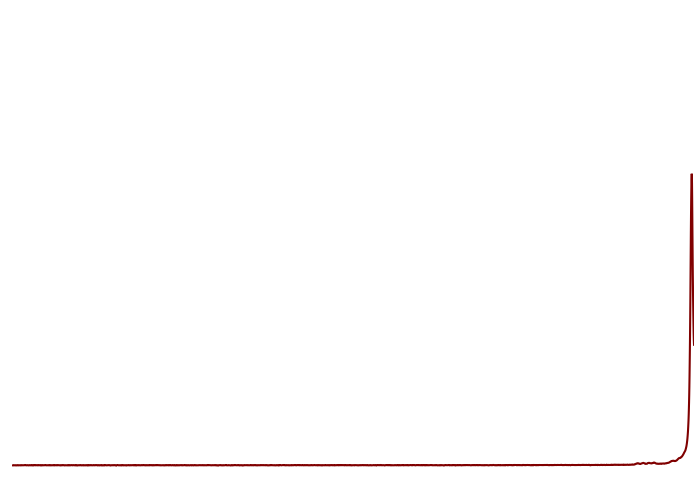

$\begin{array}{lll}9.5 & 8.0\end{array}$

7.0

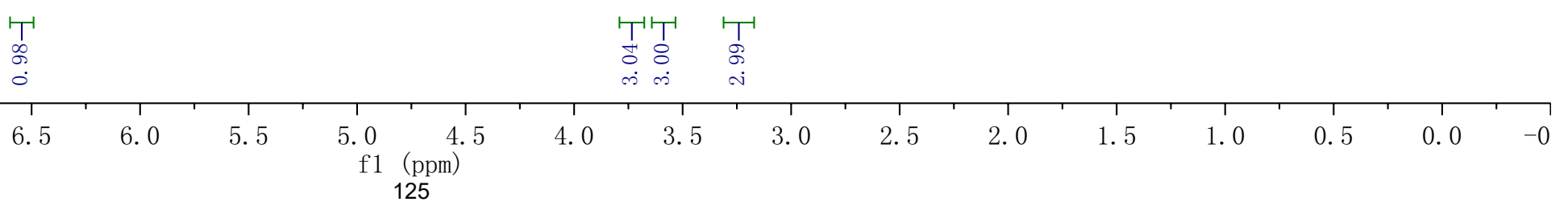




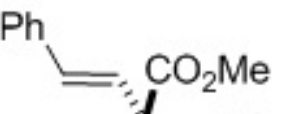

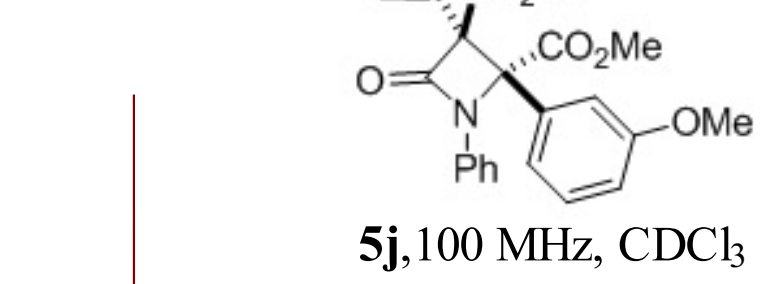

5j, $100 \mathrm{MHz}, \mathrm{CDCl}_{3}$

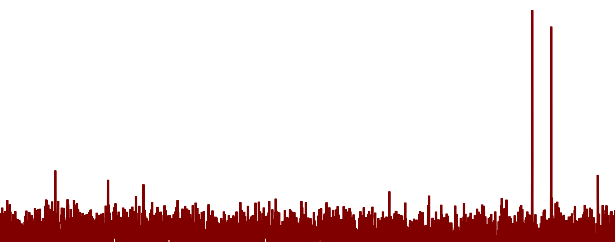

\section{sj,}

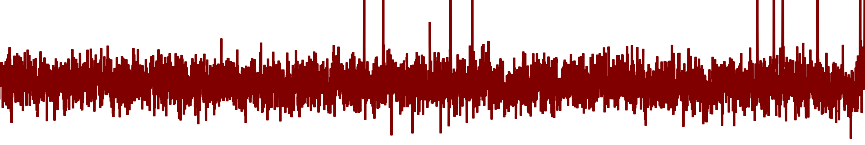


<smiles>C/C=C/[C@]1(C(=O)OC)C(=O)N(c2ccccc2)[C@]1(C(C)=O)c1ccc(C(F)(F)F)cc1</smiles>

5k,400 MHz, $\mathrm{CDCl}_{3}$
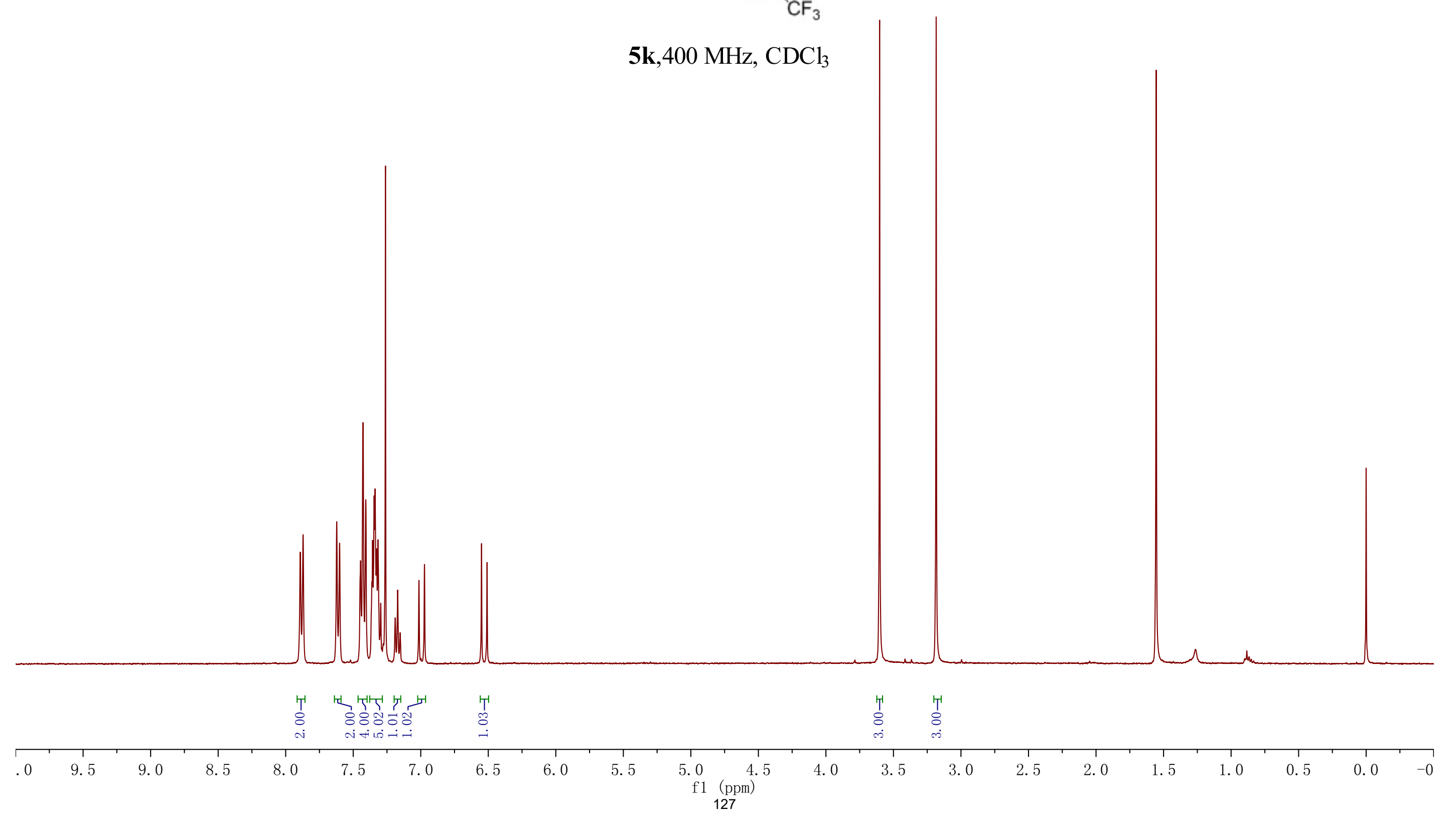
(1)

$\mathbf{5 k}, 100 \mathrm{MHz}, \mathrm{CDCl}_{3}$

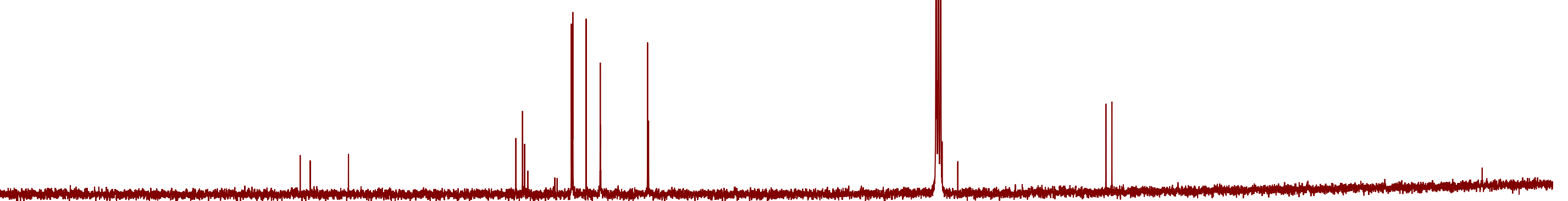

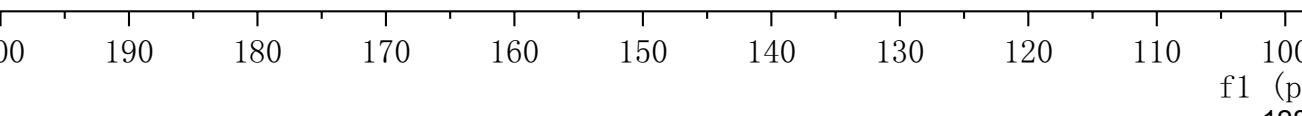




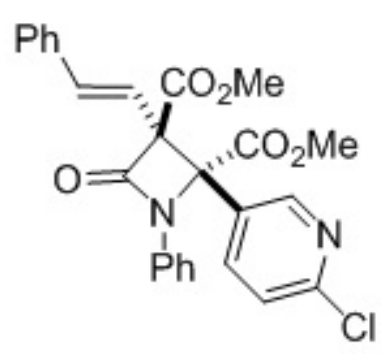

$\mathbf{5 1 , 3 0 0 ~ M H z ,} \mathrm{CDCl}_{3}$

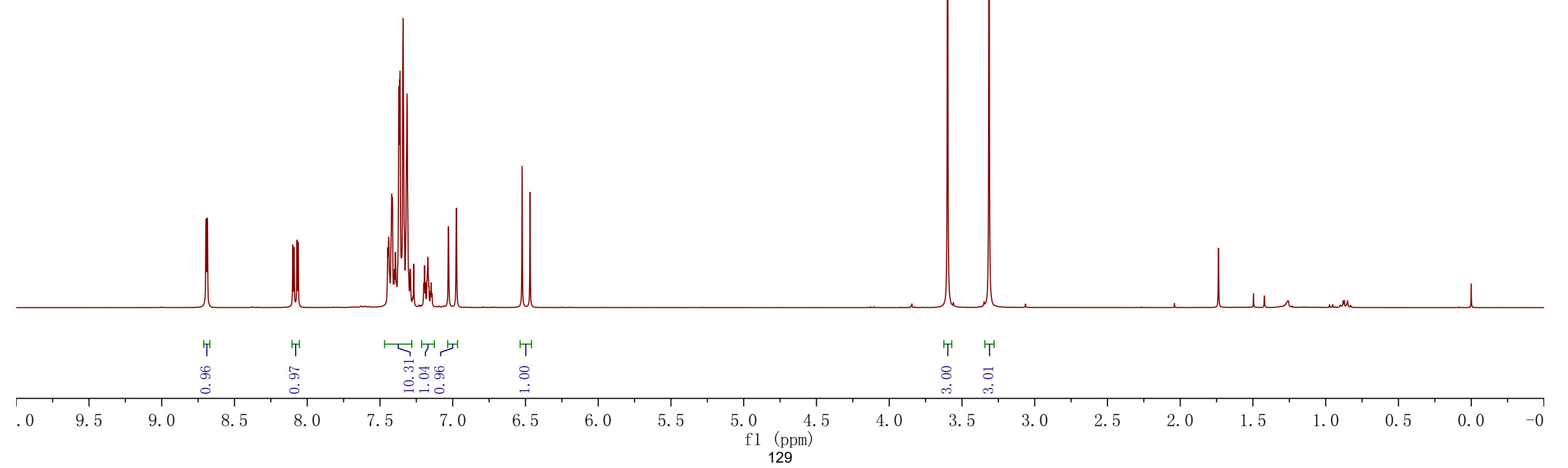


$\mathrm{Ph}$

(c)

5l,75 MHz, $\mathrm{CDCl}_{3}$ 
$\mathrm{Ph}$

$$
=\sum_{S}^{\mathrm{O}_{2} \mathrm{Me}}
$$

5m, $400 \mathrm{MHz}, \mathrm{CDCl}_{3}$

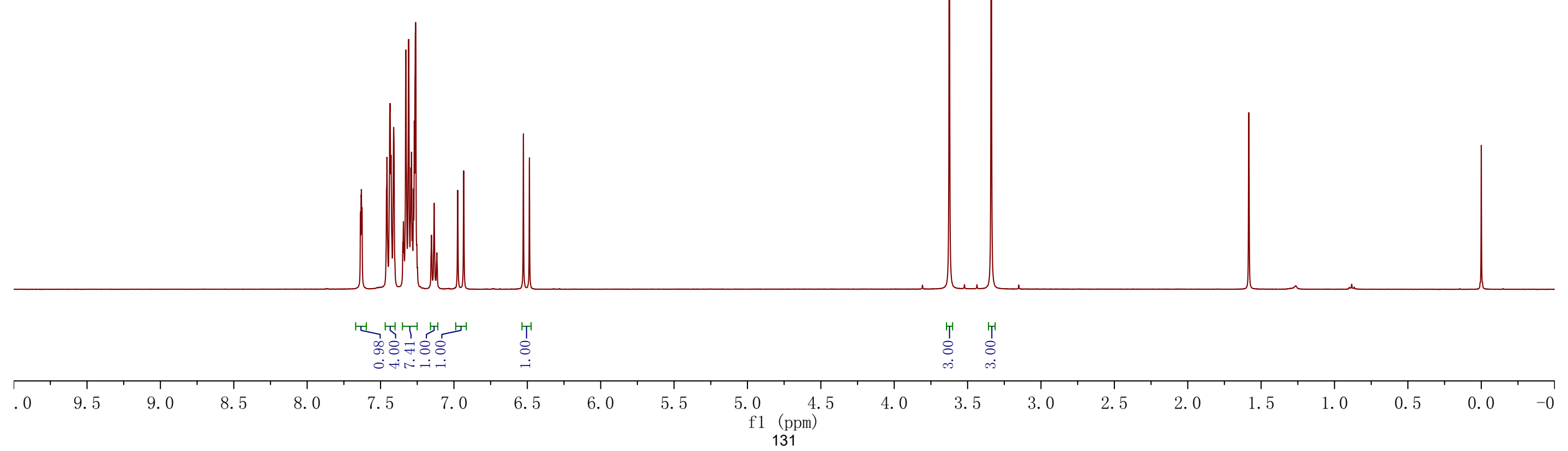




$$
\text { ( }
$$

5m, $75 \mathrm{MHz}, \mathrm{CDCl}_{3}$

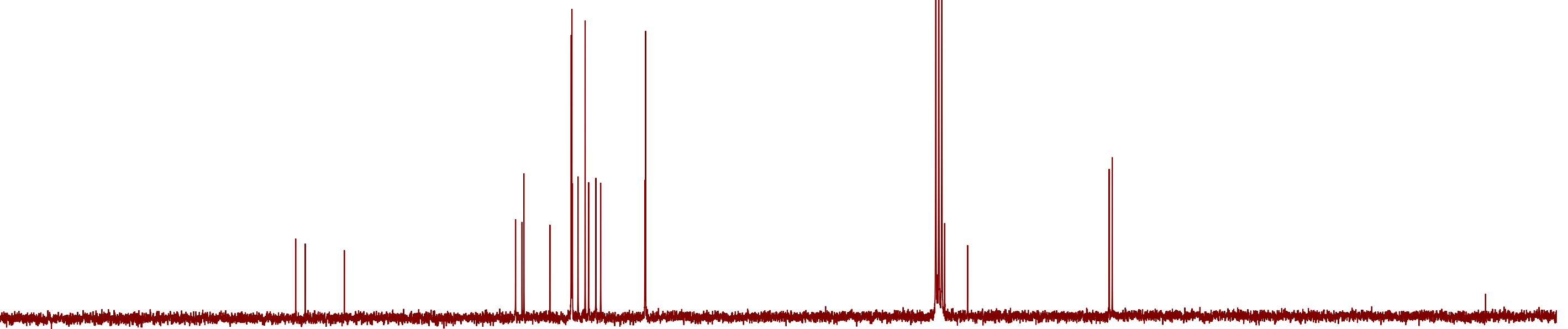




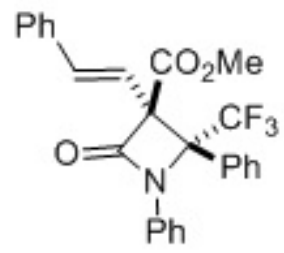

\section{5n, $400 \mathrm{MHz}, \mathrm{CDCl}_{3}$}

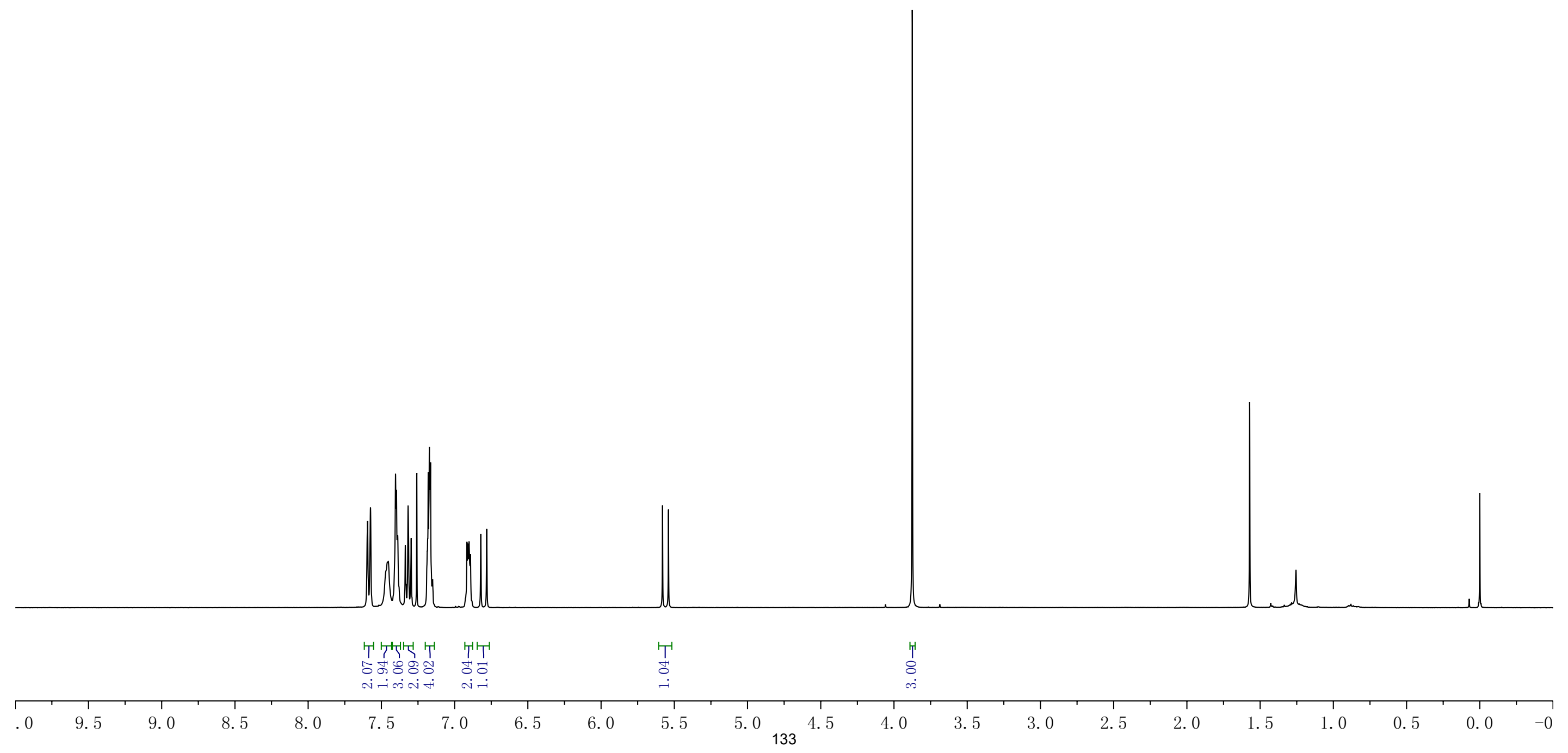




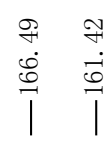
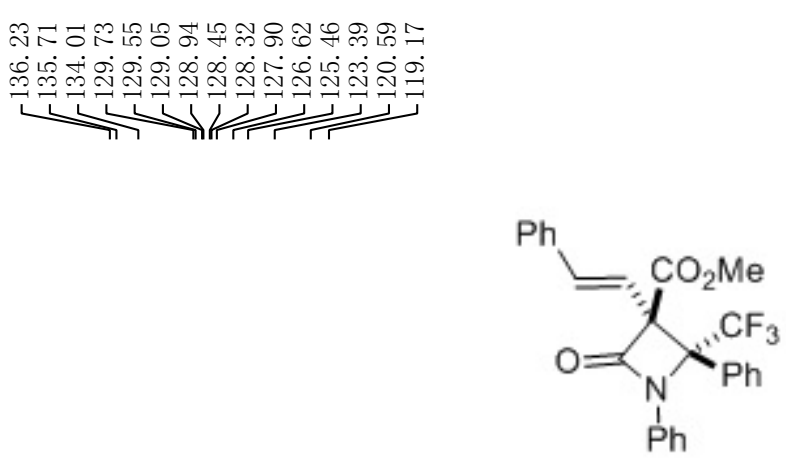

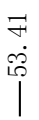

5n, $100 \mathrm{MHz}, \mathrm{CDCl}_{3}$
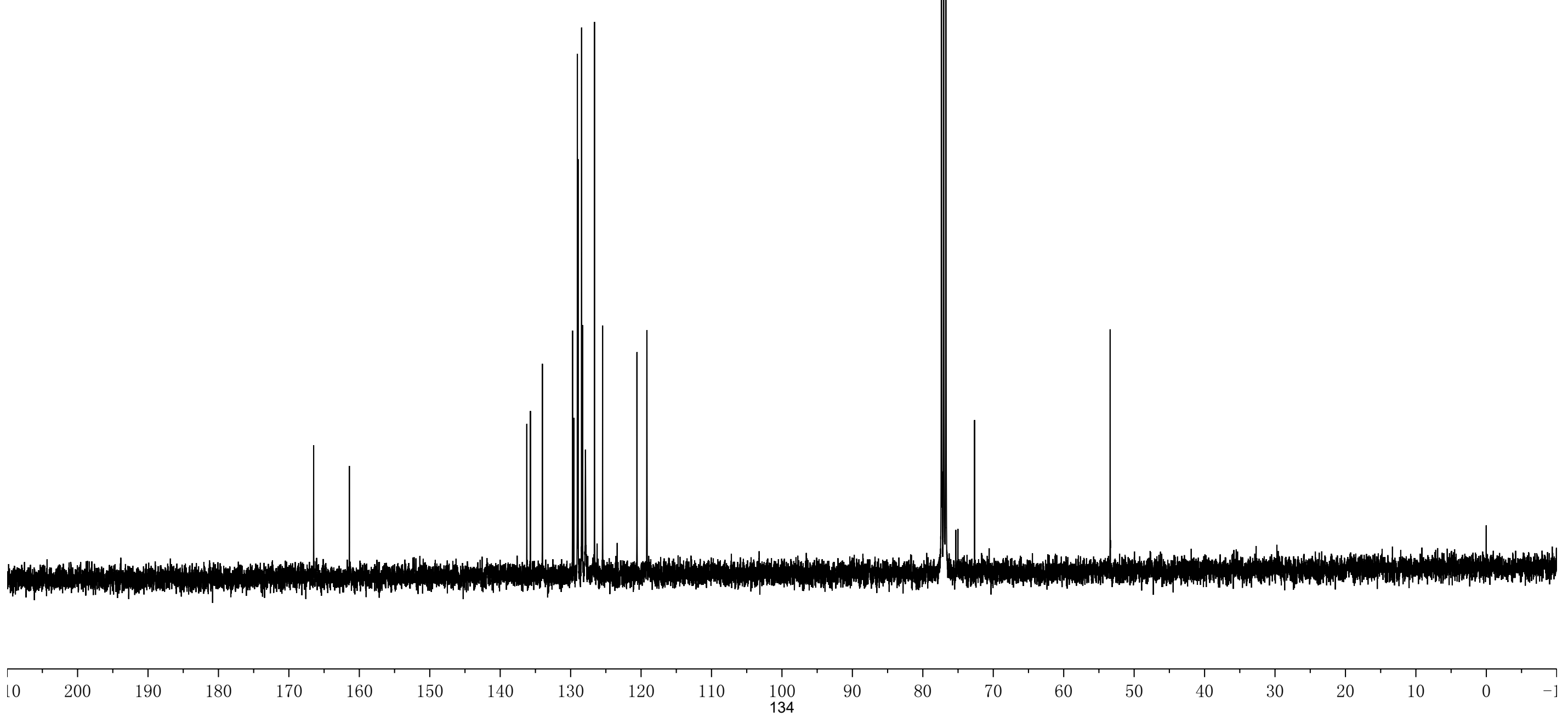
5o, $400 \mathrm{MHz}, \mathrm{CDCl}_{3}$

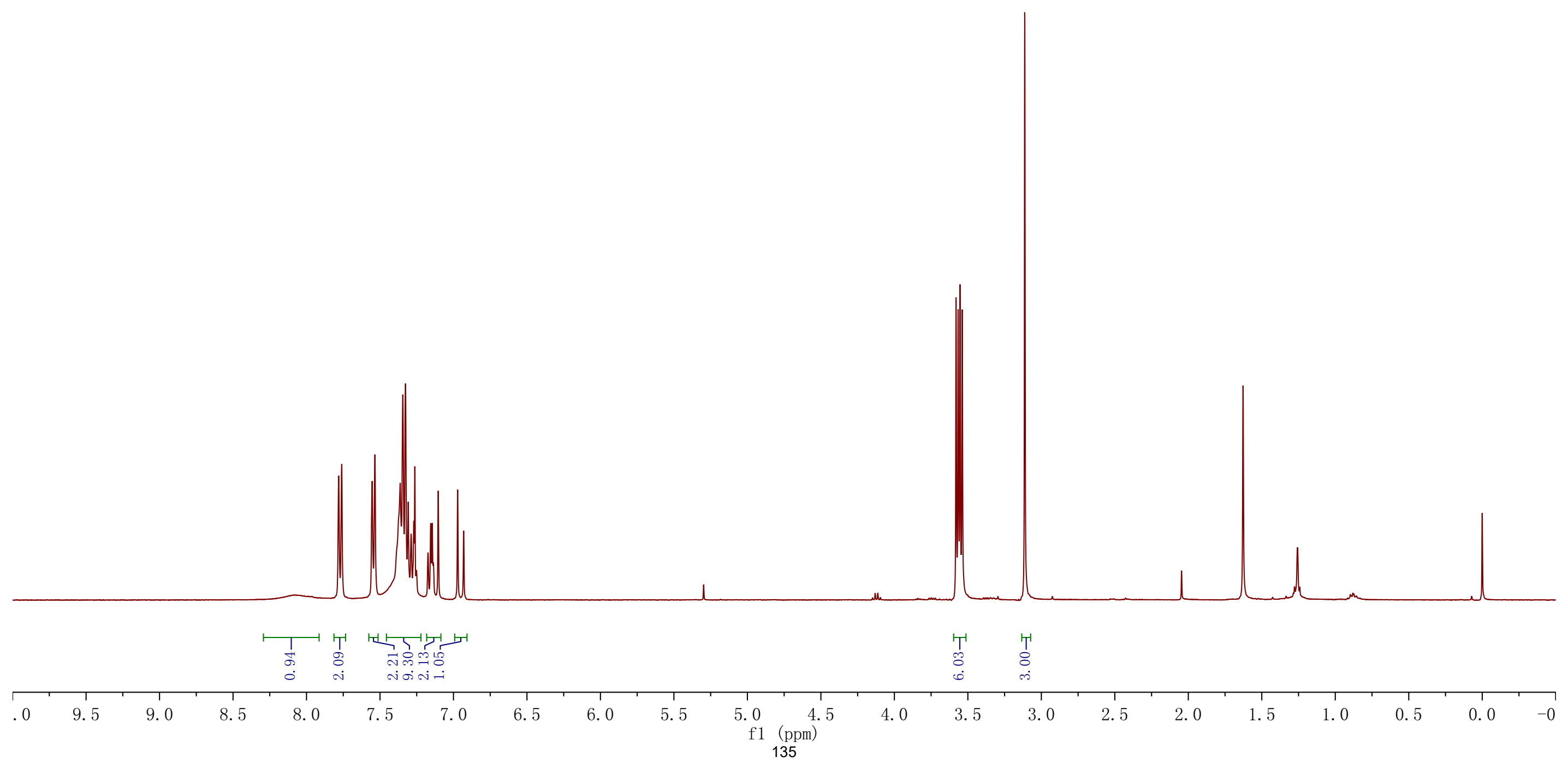




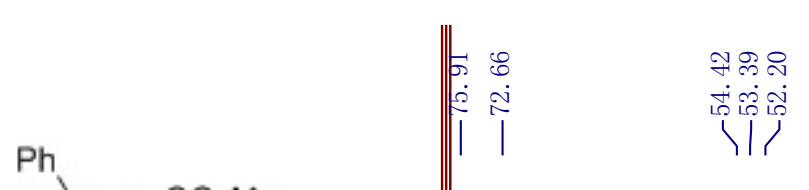

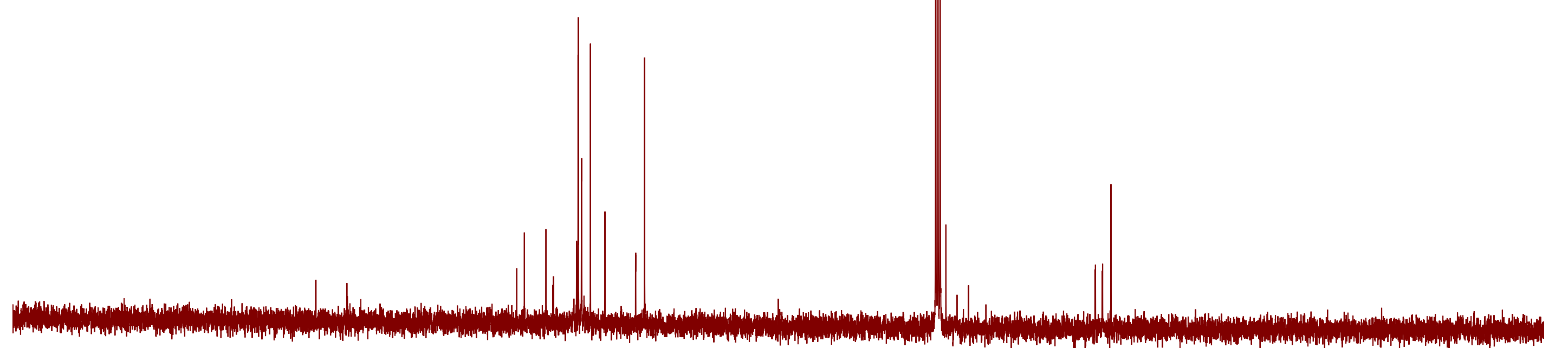

\begin{tabular}{|c|c|c|c|c|c|c|c|c|c|c|c|c|c|c|c|c|c|c|c|c|c|c|}
\hline 10 & 200 & 190 & 180 & 170 & 160 & 150 & 140 & 130 & 120 & 110 & f1 $\begin{array}{c}100 \\
136\end{array}$ & 90 & 80 & 70 & 60 & 50 & 40 & 30 & 20 & 10 & 0 & -1 \\
\hline
\end{tabular}




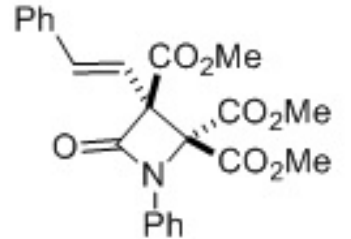

5p,400 MHz, $\mathrm{CDCl}_{3}$

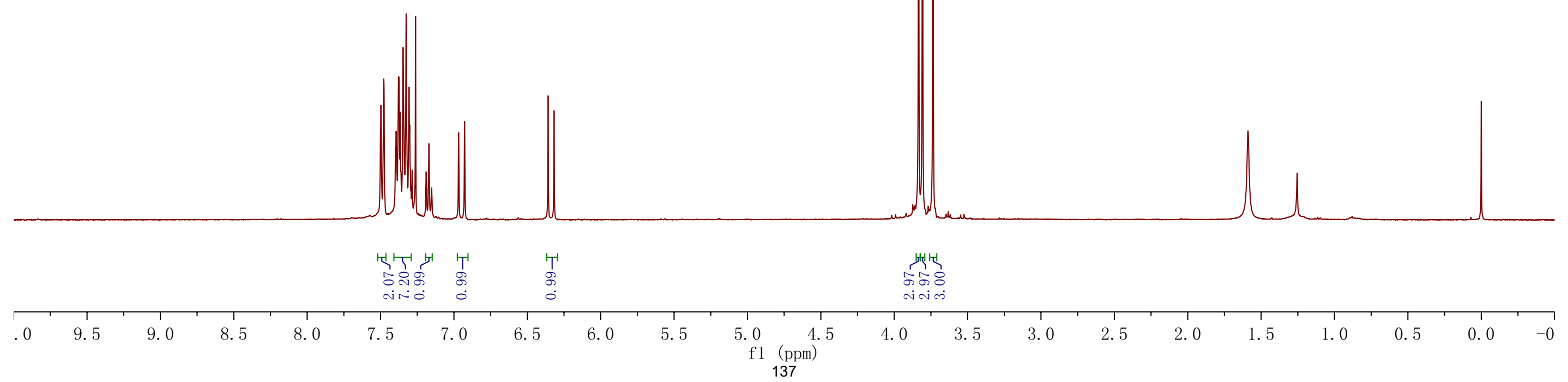




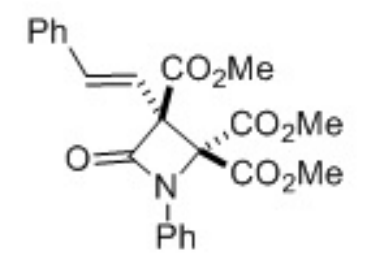

5p, $125 \mathrm{MHz}, \mathrm{CDCl}_{3}$

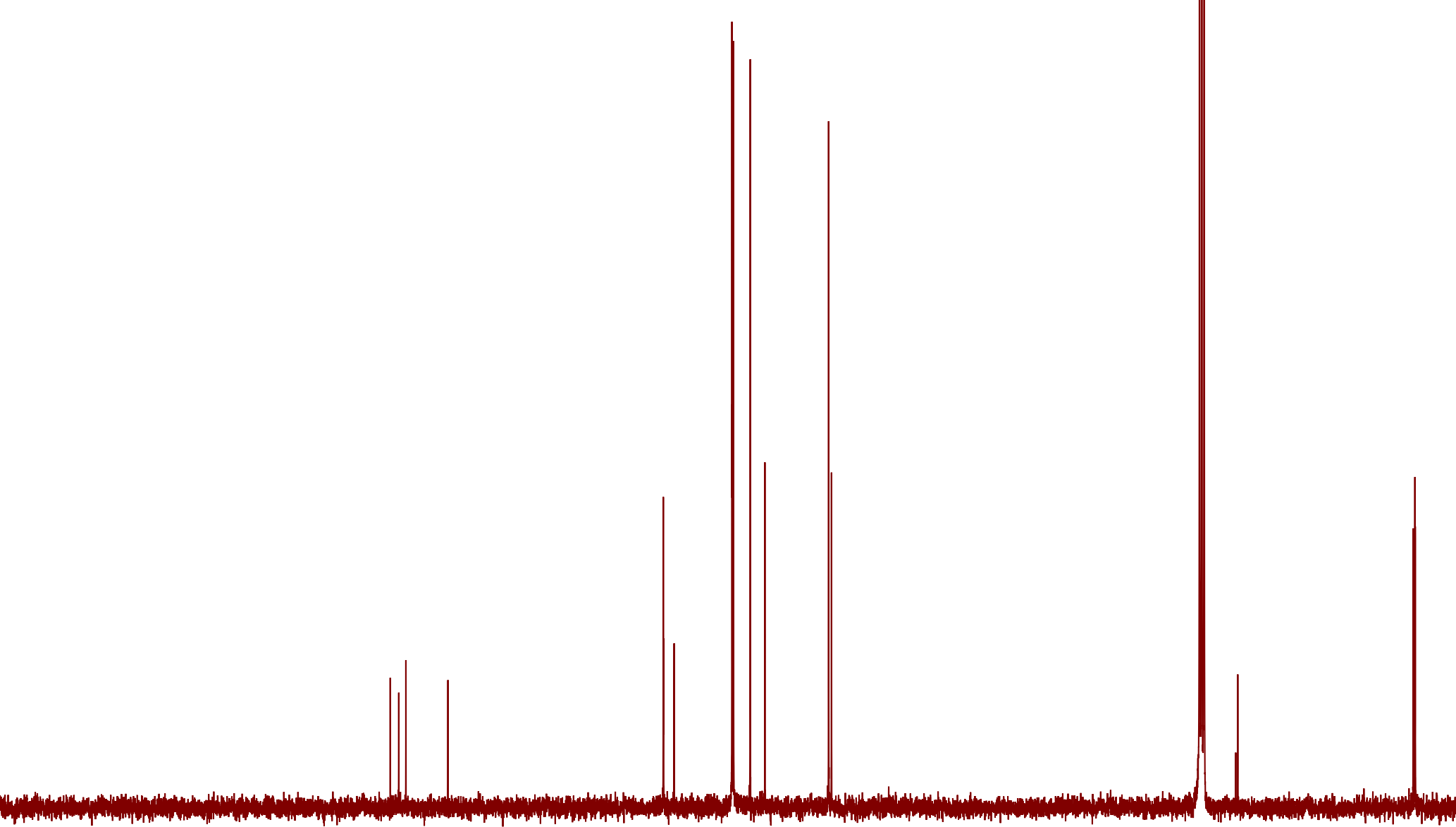



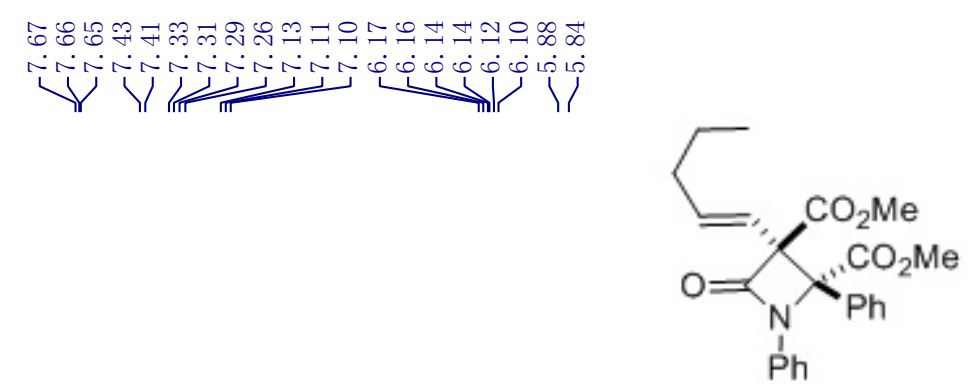

5q, $400 \mathrm{MHz}, \mathrm{CDCl}_{3}$

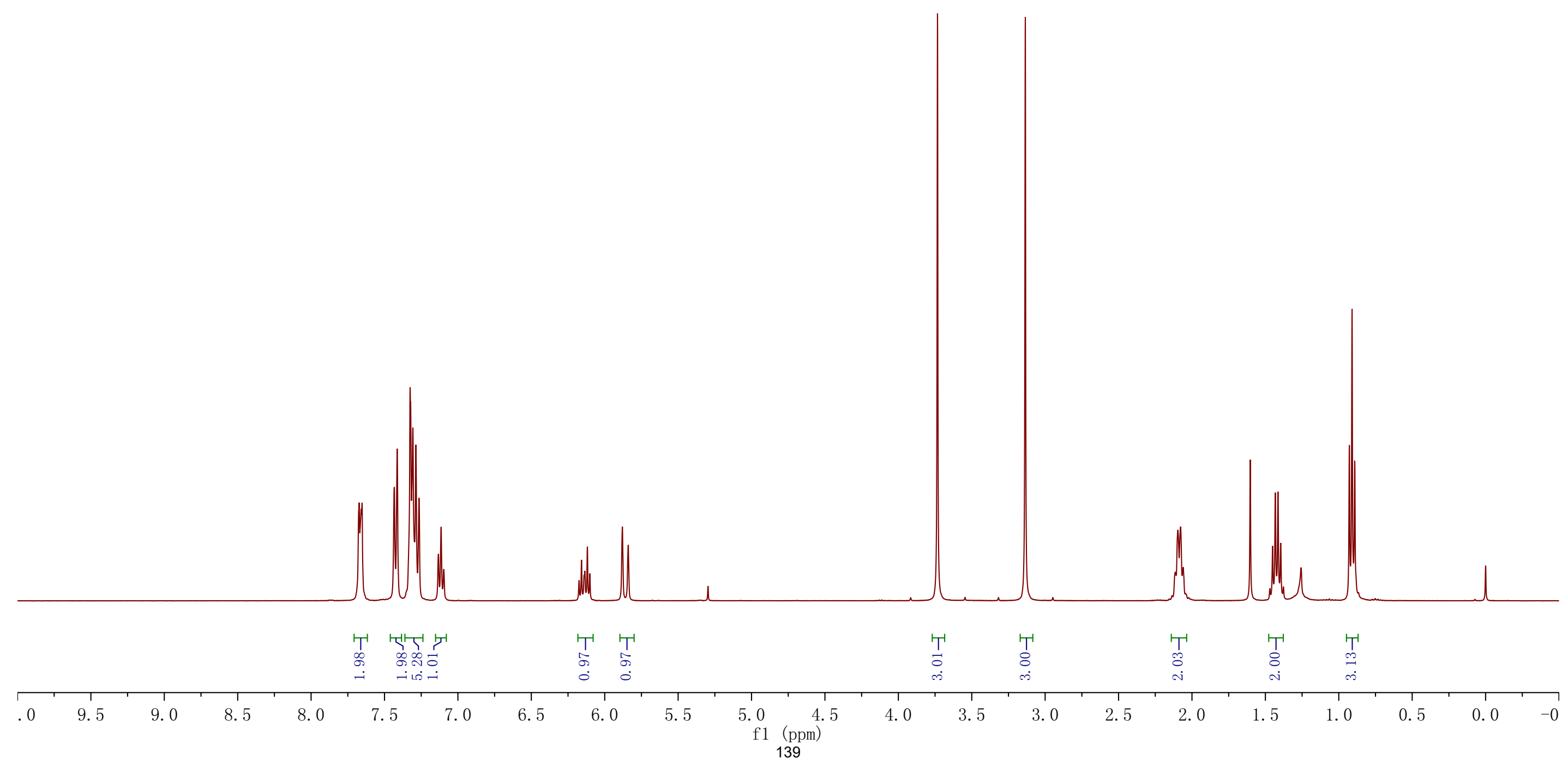




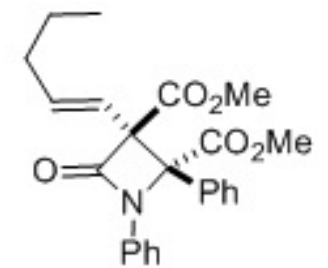

5q, $100 \mathrm{MHz}, \mathrm{CDCl}_{3}$

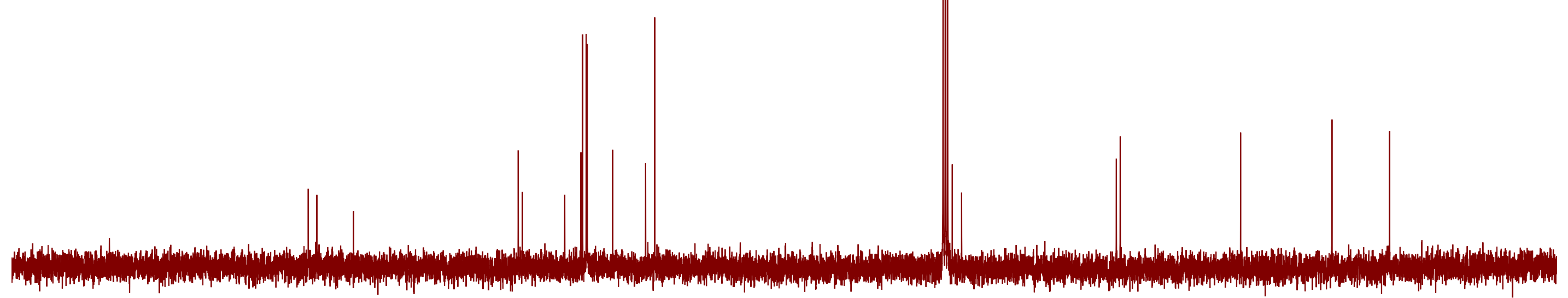




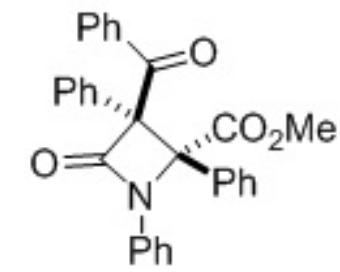

$5 \mathbf{r}, 400 \mathrm{MHz}, \mathrm{CDCl}_{3}$

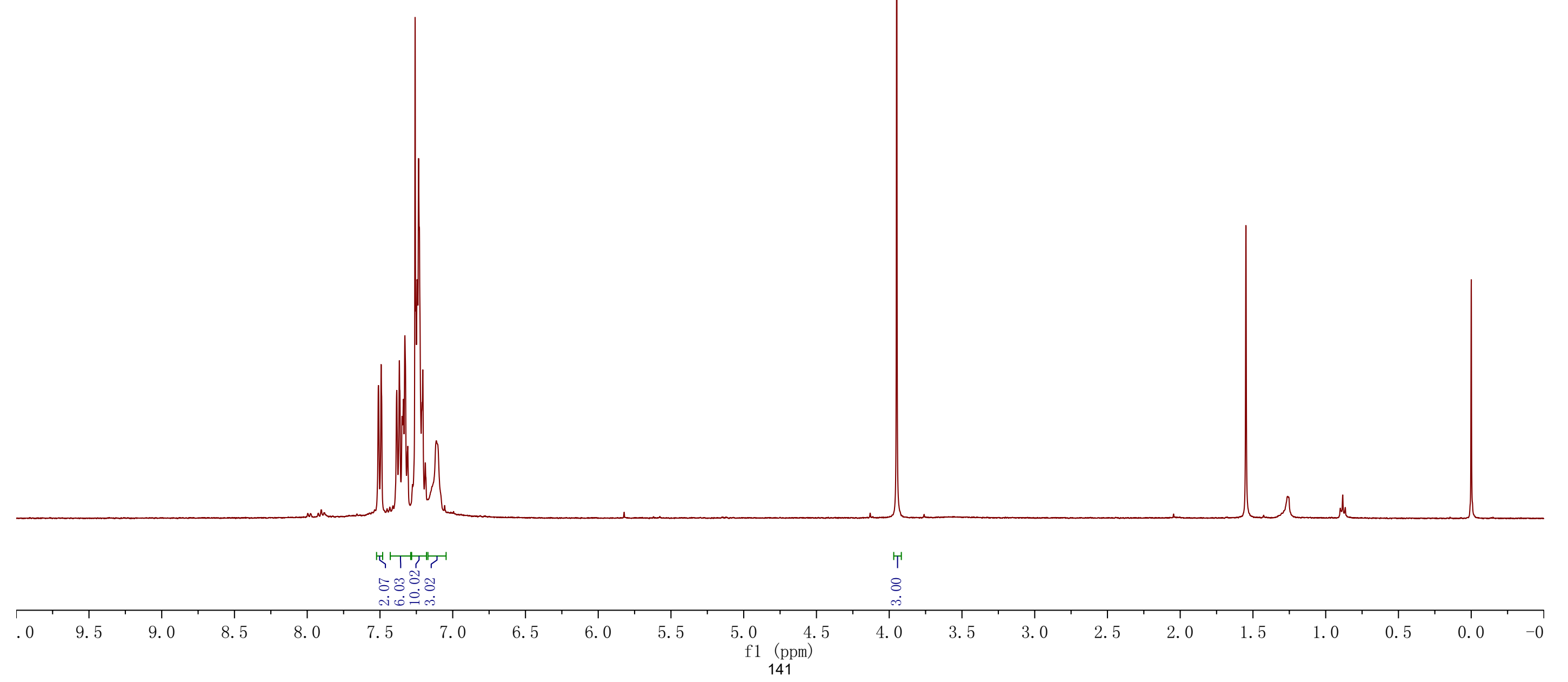


5r, $125 \mathrm{MHz}, \mathrm{CDCl}_{3}$

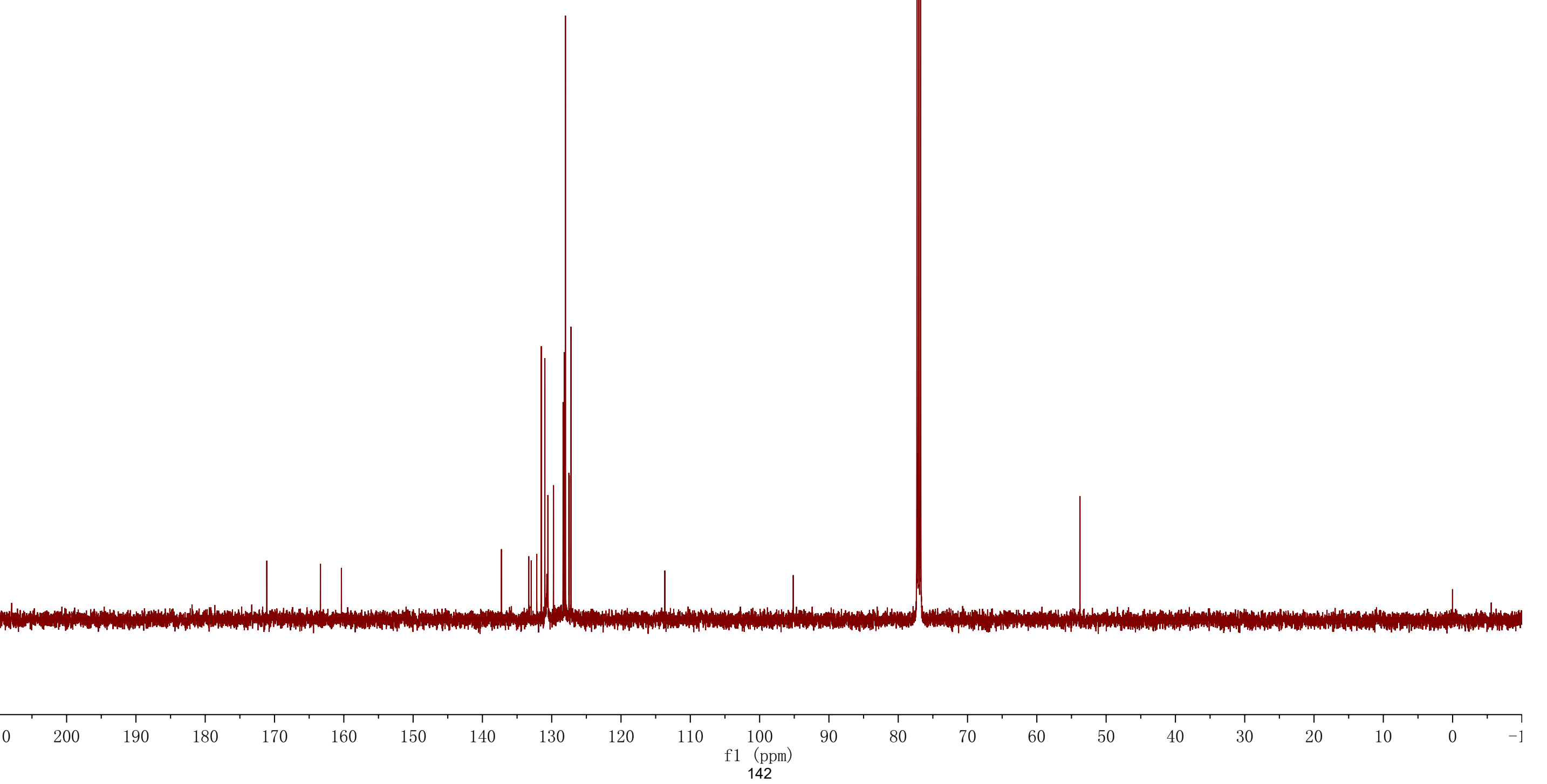




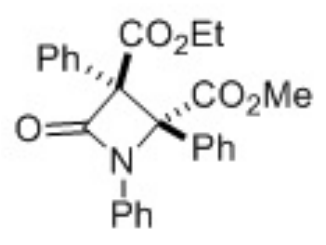

5s, $400 \mathrm{MHz}, \mathrm{CDCl}_{3}$

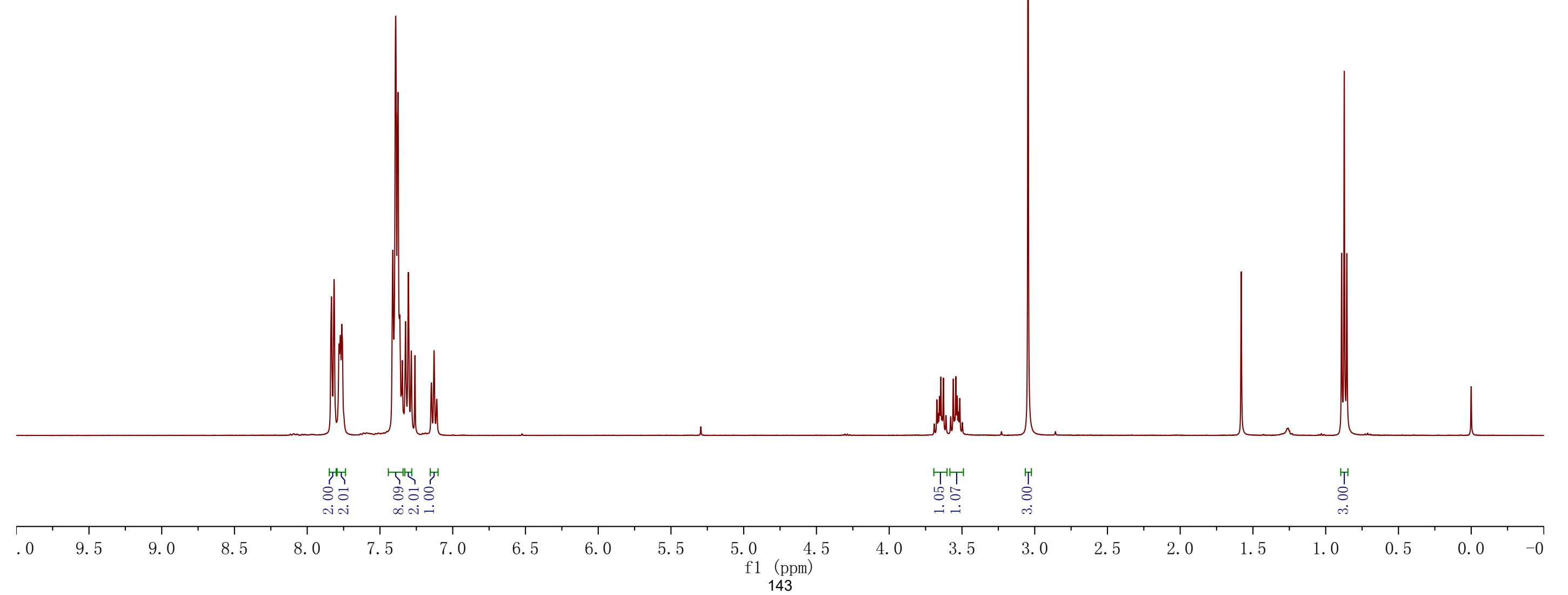


$5 \mathbf{s}, 100 \mathrm{MHz}, \mathrm{CDCl}_{3}$

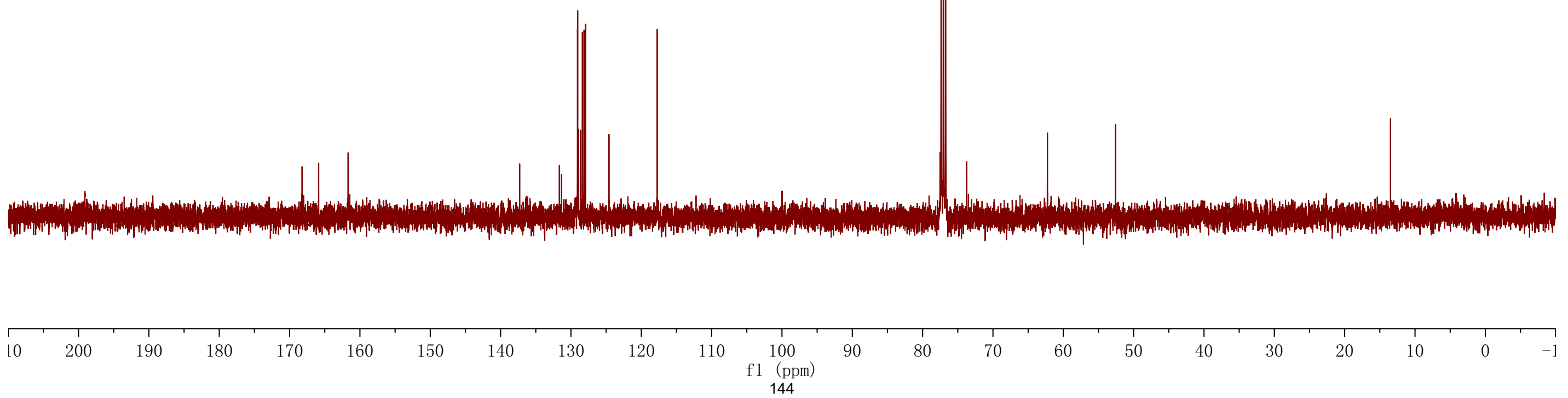

